The Portland Disposal

Site Capping Demonstration

Project

$1995-1997$

\title{
Disposal Area
}

Monitoring System

DAMOS
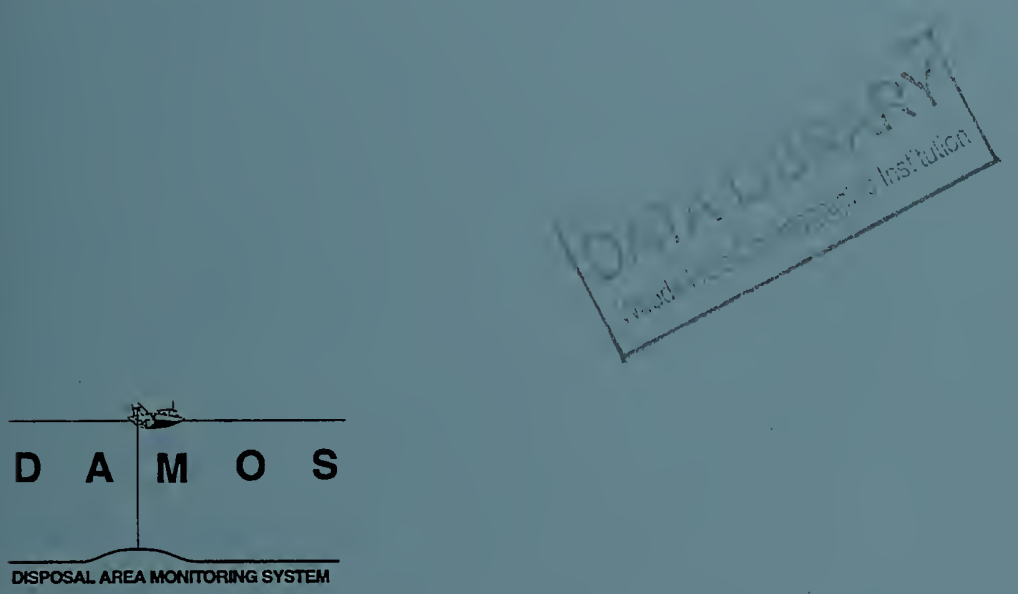

Contribution 123

September 1998

TC

187

057

no. 123

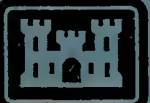

US Army Corps

of Engineers:

New England District 
Public reporting concern for the collection of information is estimated to average 1 hour per response including the time for reviewing instructions, searching existing data sources, gathering and measuring the data needed and correcting and reviewing the collection of information. Send comments regarding this burden estimate or any other aspect of this collection of information including suggestions for reducing this burden to Washington Headquarters Services, Directorate for information Observations and Records, 1215 Jefferson Davis Highway, Suite 1204 , Arlington VA $22202-4302$ and to the Office of Management and Support, Paperwork Reduction Project (0704-0188), Washington, D.C. 20503.

\begin{tabular}{|l|l|l|}
\hline 1. AGENCY USE ONLY (LEAVE BLANK) & $\begin{array}{c}\text { 2. REPORT DATE } \\
\text { September } 1998\end{array}$ & $\begin{array}{c}\text { 3. REPORT TYPE AND DATES COVERED } \\
\text { FINAL REPORT }\end{array}$ \\
\hline
\end{tabular}

4. TITLE AND SUBTITLE

THE PORTLAND DISPOSAL SITE CAPPING DEMONSTRATION PROJECT 1995-1997

5. FUNDING NUMBERS

\section{AUTHOR(S)}

JOHN T. MORRIS , HEATHER L. SAFFERT AND PEGGY MYRE MURRAY

7. PERFORMING ORGANIZATION NAME(S) AND ADDRESS(ES)

Science Applications International Corporation

221 Thrid Street

Newport, RI 02840

\section{PERFORMIGORGANIZATION REPORT NUMBER}

$$
\text { SAIC-402 }
$$

10. SPONSORING/MONITORIYG; AGENCY REPORT NUMBER
DAMOS Contribution No. 123 US Army Corps of Engineers-New England District 696Virginia Rd Concord, MA 01742-2751

9. SPONSORING/MONITORING AGENCY NAME(S) AND ADDRESS(ES)

LEMENTARY NOTES

Available from DAMOS Program Manager, Regulatory Division

USACE-NAE , 696 Virginia Rd, Concord, MA 01742-2751

\section{2a. DISTRIBUTION/AVALABULTY STATEMENT}

Approved for public release; distribution unlimited

12b. DISTRIBUTION CODE

\section{ABSTRACT}

Sediments from the Royal River in Maine, considered suitable for open-ocean disposal, were sequentially dredged and disposed at the Portiand Disposal Site (PDS) as a proof-of-concept that dredged material could be placed, and capped, in a deep water open-ocean disposal site. Monitoring protocols developed through the Disposal Area Monitoring (DAMOS) Program were utilized, as well as a newly developed tracer technique to track different lithologies of dredged material on the seafloor. Overall, the Portiand Disposal Site Capping Demonstration Project sbowed that dredged material can be effectively placed, capped, and monitored at a deep water disposal site.

Disposal and capping of dredged material is a management tecknique for the containment of sediments considered unsuitable for open-ocean disposal (unacceptably contaminated dredged material, or UDM) that bas proven successful in Long Island Sound, in relatively shallow water (approximately $20 \mathrm{~m}$ ) and over a flat seafloor. Capping at deep water disposal sites $(>40 \mathrm{~m}$ ) was an unproven management method due to a variery of factors, including historical difficulties in disposal barge positioning, and shortage of evidence confirming the formation of distinct UDM and capping layers. This tightly controlled, closely monitored deep-water capping project has provided evidence that the technique can be successful in deeper waters.

In order to avoid any potential adverse environmental impact from such a demonstration, material dredged from the Royal River. Yarmouth, ME, deemed suitable for unconfined open-water disposal, was used as both "pseudo-UDM" as well as capping dredged material (CDM). Finer grained sediment removed from the upper reaches of the river were designated as pseudo-UDM. Material from the lower reaches of the river, characterized by coarser grained material. was designared as the project CDM and was placed over the initial pseudo-UDM deposit as a cap. The capped disposal mound was formed within a basin fearure on the PDS seafloor at a depch of $64 \mathrm{~m}$. Afrer the completion of disposal and capping operations, the newly formed mound was surveyed and cored to confirm the existence of two distinct layers.

An important component of the Portiand Disposal Site Capping Demonstration Project was the identification of tracers within the Royal River that could be used to identify the sediment on the seafloor at the PDS. Prior to the excavation of sediment, 30 vibracores from three reaches (upper, middle, and outer) of the Royal River were collected and analyzed for a variety of potential tracers. Although no single tracer was identified that was both unique to one reach of the river and commonly observed in all collected samples, a statistical method of combining several biological and mineralogical parameters was found to be suitable for classifying the materia! types.

Monitoring at the Royal River Project Area in the southeast corner of the PDS utilized standard DAMOS techniques, including single-beam bathymetry, side-scan sonar. REMOTS $^{\star}$ sediment-profile images, as well as grab and core sampling. Results of the monitoring surveys showed that a discrete dredged material mound was detected on the seafloor within the Royal River Project Area. An accumulation of psendo-UDM was detected to the south and southeast of the disposal buoy position, located in the reiatively flatbottomed basin targeted for disposal. Sediment-profile inages and core data were key to mapping the footprint of both UDM and CDM on the seafloor.

The grab and core samples collected from the disposed dredged material were analyzed for the environmental tracers selected after analysis of Royal River Cores. The statistical tracer data were able to show a discernible difference between the CDM, pseudo-UDM. and ambient material.

The results of the demonstration project provided recommendations for furure cap monitoring projects in deep water disposal sires, including suggestions for modifications to both the monitoring protocols and to dredging and disposal operations. For areas of complex bottom topography, a higher resolution single-beam bathymetric survey grid (5 to $10-\mathrm{m}$ lane spacing) or multibeam bathymetry is required to provide more precise depth information over a wider area of seafloor.

Finally, the tracer technique that was selected demonstrated promising results in tracking dredged material at a subaqueous disposal site. Several recommendations were made to improve the method, including selecting tracers with the narrowest range in the dredging area, and sampling and analyzing the baseline (ambient and historical dredged) material prior to disposal of project material.

\section{SUBJECT TERMS}

Royal River, Portland Disposal Site (PDS), capping, unacceptably contaminated dredged material(UDM), dredging and disposal operations 



\section{THE PORTLAND DISPOSAL SITE CAPPING DEMONSTRATION \\ PROJECT \\ 1995 - 1997}

CONTRIBUTION \#123

September 1998

Report No.

SAIC 402

Submitted to:

Regulatory Branch

New England District

U.S. Army Corps of Engineers

696 Virginia Road

Concord, MA 01742-2751

Prepared by:

John T. Morris

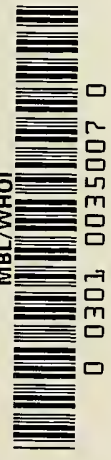

Heather L Saffert

and

Peggy Myre Murray

Submitted by:

Science Applications International Corporation

Admiral's Gate

221 Third Street

Newport, RI 02840

(401) $847-4210$ 

Page

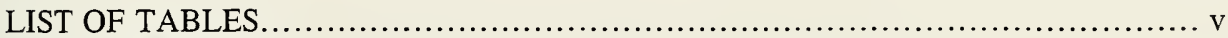

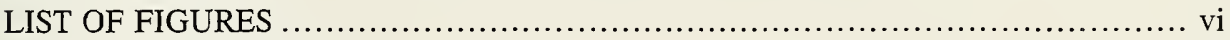

ACKNOWLEDGMENT ...........................................................

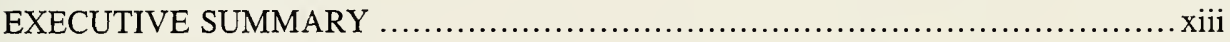

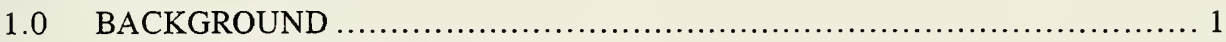

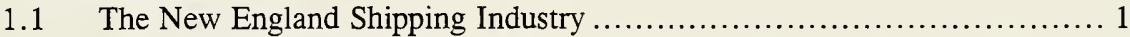

1.2 The DAMOS Program ............................................... 1

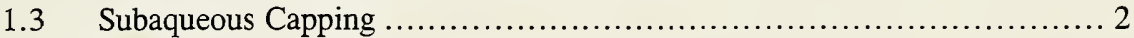

1.4 Deep-Water Capping at the Portland Disposal Site ...................... 5

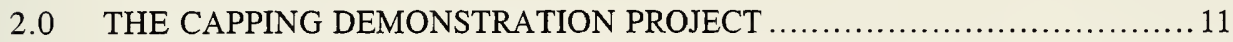

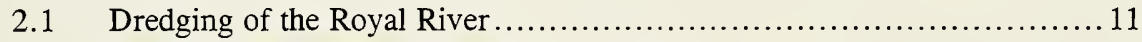

2.2 Capping Demonstration Project Time Line............................ 16

2.2.1 Baseline Surveys at the Royal River and Royal River Project Area . 16

2.2.2 Dredging Operations, Fall-Winter 1995-96 ..................... 19

2.2.3 Expanded Royal River Project Area Baseline Survey ...............23

2.2.4 Pseudo-UDM Dredging Operations, Fall 1996.................... 23

2.2.5 Royal River Project Area Precap (Pseudo-UDM) Survey ............23

2.2.6 Capping Dredging Operations, Fall-Winter 1996-97 ...............24

2.2.7 Royal River Project Area Postcap (CDM) Survey .................. 24

2.2.8 Additional Analysis of Royal River Sediment Cores ................ 24

$2.3 \quad$ Capping Model Predictions ............................................. 24

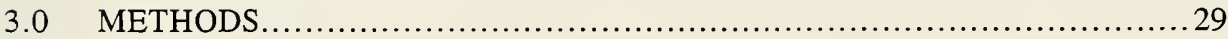

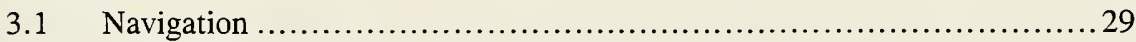

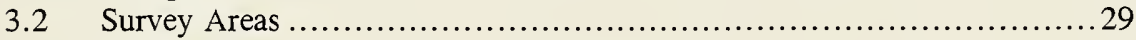

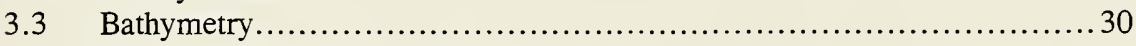

3.3.1 Bathymetric Data Collection ................................. 30

3.3.2 Bathymetric Data Processing .................................... 32

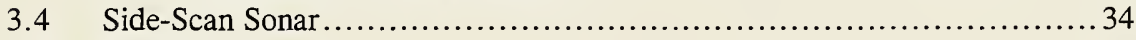

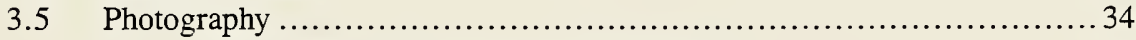

3.5.1 REMOTS ${ }^{\circledR}$ Sediment-Profile Photography ........................ 34

3.5.2 Planview Photography .......................................... 35

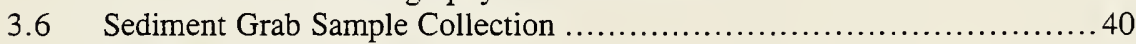

3.7 Sediment Coring Collection ............................................. 42

3.7.1 Royal River Survey ......................................... 42

3.7.2 Postcap Survey ................................................ 42

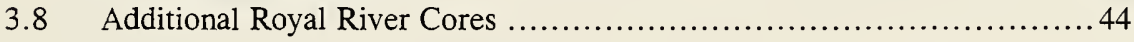


3.8.1 Selection and Processing of Additional Cores .......................44

3.8.2 Comparison of Methods of Microfossil Preservation................. 45

3.9 Tracer Analyses ...................................................... 46

3.9.1 Sewage Tracer (Royal River only) ............................ 46

3.9.2 Grain Size .................................................... 46

3.9 .3 Coarse Fraction.............................................. 46

3.9.4 Fine Fraction: Microfossil and Mineralogy Observations ............ 46

3.10 Multivariate Statistical Analyses of Fine Fraction Results ................ 47

3.10.1 Sample Selection and Database Description ..................... 48

3.10.2 Clustering and Multi-Dimensional Scaling Analysis ............... 48

3.10.3 Analysis of Similarities........................................ 49

3.10.4 Discriminant Statistical Analysis ..............................50

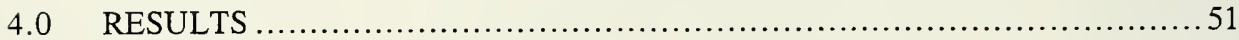

$4.1 \quad$ Royal River Sediment Coring....................................... 51

4.1.1 Sediment Characterization .................................... 51

4.1.2 Sediment Tracer Analysis ....................................5 52

4.2 Royal River Project Area August 1995 Baseline Survey .....................5 59

4.3 Expanded Royal River Project Area February 1996 Baseline Survey ........ 59

4.3 .1 Bathymetry ..................................................... 62

4.3.2 REMOTS ${ }^{\circledR}$ Sediment-Profile Photography .........................62

4.3.3 Planview Photographs .......................................... 67

4.3.4 Side-Scan Sonar ................................................. 67

4.4 Royal River Project Area Precap Survey ..............................69 69

4.4 .1 Bathymetry ..................................................... 69

4.4.2 REMOTS ${ }^{\circledR}$ Sediment-Profile Photography ......................... 74

4.4 .3 Sediment Grab Sampling ....................................... 74

4.5 Royal River Project Area Postcap Survey ............................. 87

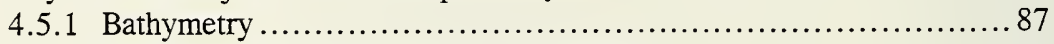

4.5.2 REMOTS ${ }^{\circledR}$ Sediment-Profile Photography ........................ 89

4.5.3 Sediment Coring ............................................... 97

4.6 Processing and Analysis of Additional Royal River Cores............... 107

4.7 Multivariate Statistical Analysis of Fine Fraction Results ................. 111

4.7.1 Clustering and Multi-Dimensional Scaling Results ............... 111

4.7.2 Analysis of Similarities....................................... 116

4.7.3 Discriminant Statistics ................................... 116

5.0 DISCUSSION........................................................ 119

5.1 Assessment of the Footprint of the Capped Disposal Mound............. 119 
5.1.1 Pseudo-Unacceptably Contaminated Dredged

Material (Pseudo-UDM

5.1.2 Capping Dredged Material (CDM) ........................... 123

5.2 Tracking Dredged Material on the Seafloor .......................... 123

5.2 .1 Evaluation of Estuary Tracers................................. 124

5.2.2 Statistical Strength of Tracer Grouping Method.................. 126

5.2.3 Differentiating Ambient from Historic Dredged Material ......... 127

5.3 Evaluation of Tools.............................................. 129

5.3 .1 Bathymetry ................................................ 129

5.3.2 Photography: Planview and REMOTS ${ }^{\circledR}$ Sediment-Profile Imaging 129

5.3.3 Grab Sampling and Coring ................................... 129

5.3.4 Fine Fraction Tracer Technique ............................ 130

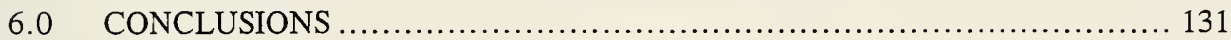

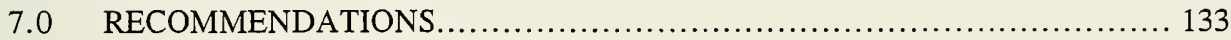

7.1 Bathymetric Data Collection ....................................... 133

7.2 Operational Control ................................................ 133

7.3 Sediment Tracer Technique ..................................... 134

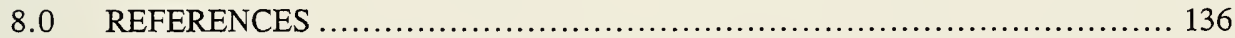

\section{APPENDICES}

INDEX 



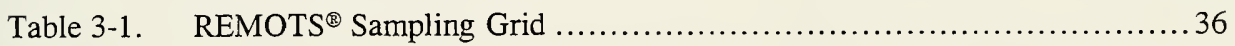

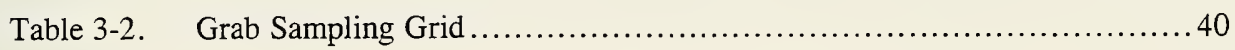

Table 3-3. Royal River Core Data......................................... 43

Table 3-4. PDS Gravity Cores ............................................. 44

Table $4-1 . \quad$ Core Fraction Results .......................................... 54

Table 4-2. Royal River Core Sample Fine Fraction Mineralogy Results..............58

Table 4-3. November 1996 Precap REMOTS ${ }^{\circledR}$ Sediment-Profile Photography

Survey Results Showing Detection of Fresh Pseudo-UDM and

Historic Dredged Material .......................................... 80

Table 4-4. Precap (UDM) Grab Sample Survey Grain Size Analysis Results...........83

Table 4-5. Precap (Pseudo-UDM) Grab Sample Fine Fraction Mineralogy Results ........................................................ 83

Table 4-6. January 1997 Postcap REMOTS ${ }^{\circledR}$ Survey Results Showing of Cap

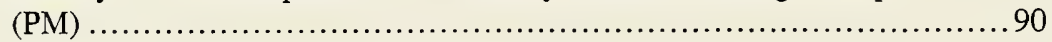

Table 4-7. Postcap (CDM) Sediment Coring Survey Grain Size Analysis Results .... 100

Table 4-8. Postcap Core Sample Fine Fraction Mineralogy Results ............... 103

Table 4-9. Sample Identification for Statistical Analyses ....................... 113 

Page

Figure 1-1. Graphical representation of maintenance dredging operations within an industrialized harbor in New England...

Figure 1-2. Geographic locations of the ten DAMOS disposal sites along the coast of New England.

Figure 1-3. Geographic location of major marine terminals and public works facilities associated with urbanization (Custom Communications 1995) ..... 8

Figure 1-4. Portland Harbor Characterization Study sample locations within the Fore River

Figure 2-1. Topographic map of a portion of Cumberland County, ME and the Royal River watershed area

Figure 2-2. Process map of the Royal and Cousin river systems (MSPO 1983 13

Figure 2-3. Photographs at Yarmouth Harbor: A. Ship under construction, west bank, circa 1875, and B. Status of the harbor circa 1915 (Attanas and Hinkley 1997)

Figure 2-4. Royal River, ME showing navigation channel and anchorage areas dredged for the Capping Demonstration Study

Figure 2-5. Example of a channel cross-section used to graphically display the riverbed and estimate volumes of material to be removed from the channel

Figure 2-6. Time line of events within the Portland Disposal Site Capping Demonstration Project

Figure 2-7. Examples of specimens found within the Royal River estuary and expected habitat ranges and distribution

Figure 2-8. Bathymetric chart of the $1950 \mathrm{~m} \times 1875 \mathrm{~m}$ master survey (NAD 27) performed over PDS in December 1977, $2.0 \mathrm{~m}$ contour interval (MLLW) 
Figure 2-9. Map of Royal River and PDS showing core locations and areas of dredged material origin and eventual deposition........................26

Figure 2-10. Comparison of a typical survey lane over the Central Long Island Sound Disposal Site versus the Portland Disposal Site and how gridding and differences in survey vessel track tend to affect the bathymetric data collection

Figure 3-1. Base map showing the $1950 \mathrm{~m} \times 1000 \mathrm{~m}$ bathymetric survey area during the February 1996 baseline survey (NAD 27) in relation to the Royal River Project Area (yellow) at PDS, $2.0 \mathrm{~m}$ contour interval

Figure 3-2. Comparison of the two types of tidal data collected as part of the February 1996 baseline bathymetric survey

Figure 3-3. Graphical depiction of the REMOTS ${ }^{\circledR}$ sediment-profile camera and the images it collects

Figure 3-4. Bathymetric chart of the Royal River Project Area (NAD 27) showing REMOTS $^{\circledR}$ sediment-profile station locations relative to the disposal site boundary

Figure 3-5. Examples of corresponding REMOTS ${ }^{\circledR}$ and planview images collected at station CTR as part of the February 1996 baseline survey

Figure 3-6. Bathymetric chart of the Royal River Project Area (NAD 27) showing locations of grab sampling and gravity core stations

Figure 4-1. Histogram comparing mean abundance and particle size for the mineralogical and biological components utilized as sediment tracer elements

Figure 4-2. Histograms comparing relative abundance and density of microfossils used as sediment tracer elements, see text for definition of microorganisms

Figure 4-3. Bathymetric chart of the August $1995800 \mathrm{~m} \times 800 \mathrm{~m}$ Royal River Project Area (NAD 27) relative to the disposal site boundaries, $1.0 \mathrm{~m}$ contour interval (MLLW) 60 
Page

Figure 4-4. Three-dimensional view of the August 1995 Royal River Project Area (NAD 27) depicting natural features in the central disposal location, $1.0 \mathrm{~m}$ contour interval (MLLW)

Figure 4-5. Bathymetric chart of the February $19961950 \mathrm{~m} \times 1000 \mathrm{~m}$ survey area (NAD 27) over the southern region of Portland Disposal Site relative to the project area and disposal area boundary, $2.0 \mathrm{~m}$ contour interval (MLLW)

Figure 4-6. Three-dimensional view of the February 1996 survey area over the Portland Disposal Site depicting the rough, irregular bottom topography, $1.0 \mathrm{~m}$ contour interval.

Figure 4-7. Characterization of ambient sediments and dredged material within the Royal River Project Area, as detected by the February 1996 REMOTS $^{\circledR}$ camera survey

Figure 4-8. $\quad$ REMOTS ${ }^{\circledR}$ photographs collected from stations $100 \mathrm{NW}$ and $50 \mathrm{NW}$ during the February 1996 survey showing the appearance and thickness of Harraseeket River material.

Figure 4-9. Planview images obtained from stations $100 \mathrm{NW}, \mathrm{CTR}$, and $50 \mathrm{SE}$ showing differences in surface conditions and sediments in close proximity to the central disposal point 68

Figure 4-10. Results from the side-scan sonar survey performed over the southern region of the Portland Disposal Site in February 1996

Figure 4-11. Side-scan sonar data from February 1996 survey lanes 20 and 24 overlaid with bathymetry showing rapid changes in depth due to bedrock outcrops

Figure 4-12. Depth difference plot of the November 1996 UDM survey versus the February 1996 baseline survey (NAD 27) showing apparent accumulation of pseudo-UDM in close proximity to the PDA buoy, $0.25 \mathrm{~m}$ contour interval 
Figure 4-13. Three-dimensional view of the $800 \mathrm{~m} \times 800 \mathrm{~m}$ survey area over Portland Disposal Site, showing patterns of accumulation and survey artifacts with respect to bathymetric features

Figure 4-14. REMOTS $^{\circledR}$ photographs collected during the November 1996 UDM survey showing color and consistency of Royal River dredged material (pseudo-UDM)

Figure 4-15. Thickness of CDM measured by REMOTS ${ }^{\circledR}$ and core data overlaid on a postcap bathymetric chart of (NAD 27), $1.0 \mathrm{~m}$ contour interval

Figure 4-16. REMOTS $^{\circledast}$ photographs collected at Stations $100 \mathrm{~N}$ and $200 \mathrm{~W}$ showing multiple sediment strata visible in the top $20 \mathrm{~cm}$

Figure 4-17. REMOTS $^{\circledR}$ photographs collected at Station $300 \mathrm{SE}$ and $100 \mathrm{~N}$ showing evidence of rapid recolonization one week after postdisposal .....78

Figure 4-18. Mean grain size analysis results for precap (UDM) grab samples and postcap (CDM) core samples

Figure 4-19. Histogram displaying mean mineralogical sediment composition within the fine fraction.

Figure 4-20. Histograms showing relative abundance and density of microfossils collected within the ten processed grab samples (methanol [ME] and formalin $[\mathrm{F}]$ at $50 \mathrm{NE}$ only) 86

Figure 4-21. Depth difference comparison of the January 1997 CDM survey versus the February 1996 baseline survey relative to the pseudo-UDM footprint (red) 88

Figure 4-22. Thickness of pseudo-UDM measured by REMOTS ${ }^{\circledR}$ and core data overlaid on a postcap bathymetric chart (NAD 27), 1.0 m contour interval

Figure 4-23. REMOTS $^{\circledR}$ photographs collected at Stations $200 \mathrm{~S}$ and $200 \mathrm{NW}$ as part of the January 1997 CDM survey showing multiple sediment strata within the top $20 \mathrm{~cm}$ 
Figure 4-24. REMOTS ${ }^{\circledR}$ photographs collected at Stations $100 \mathrm{~W}$ and $200 \mathrm{SW}$ as part of the January 1997 CDM survey showing the distinct coarsegrained sand over pseudo-UDM..................................... 95

Figure 4-25. REMOTS ${ }^{\circledR}$ photographs collected at Stations $50 \mathrm{~N}$ and $50 \mathrm{SW}$ as part of the January 1997 CDM survey showing the composition of the finer-grained CDM detected at several stations ......................... 96

Figure 4-26. REMOTS $^{\circledR}$ photographs collected at Stations CTR and $50 \mathrm{~N}$ as part of the January 1997 CDM survey showing evidence of the estuarine origin and biologic activity in the dredged material...................... 98

Figure 4-27. Depth difference plot showing detectable capped mound deposit (NAD 27) complete with gravity coring station locations

Figure 4-28. Histogram of the mean mineralogy composition of the UDM survey grab samples and the PDS core, CDM, UDM and ambient layers 104

Figure 4-29. Histograms of mean relative abundance and density of microfossils in the sediment samples analyzed as part of the Royal River Capping Demonstration Project

Figure 4-30. Histograms of mineralogical composition of the three additional Royal River cores 108

Figure 4-31. Histograms of relative abundance of microfossils and the numbers of individuals counted for the given number of trays analyzed for additional Royal River cores

Figure 4-32. A. Dendrogram of hierarchical agglomerate clustering using BrayCurtis similarity index of microfossil data from PDS cores 114

Figure 4-33. A. Dendrogram of hierarchical agglomerate clustering using BrayCurtis similarity index of mineralogy data from PDS cores

Figure 4-34. Plots of mineralogy and microfossil discriminant scores showing distinct clusters of samples according to layers in PDS cores

Figure 5-1 Thickness of pseudo-UDM measured by REMOTS and core data overlaid on a postcap bathymetric chart (NAD 27) 
Figure 5-2 Thickness of CDM measured by REMOTS and core data overlaid on

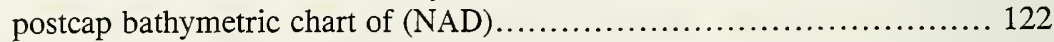

Figure 5-3. Mineralogical and biological tracers with narrowest ranges observed in Royal River cores 
Science Applications International Corporation (SAIC) and the New England District of the U.S. Army Corps of Engineers (NAE) would like to extend their gratitude to the owners and operators of Yankee Marine as well as the Town of Yarmouth, Maine for their assistance with a variety of issues associated with the dredging operations in Royal River and the Portland Disposal Site Capping Demonstration Project. Partial funding for this study was provided by U.S. EPA Region I and the Casco Bay Estuary Project. 
Sediments from the Royal River in Maine, considered suitable for open-ocean disposal, were sequentially dredged and disposed at the Portland Disposal Site (PDS) as a proof-of-concept that dredged material could be placed, and capped, in a deep water openocean disposal site. Monitoring protocols developed through the Disposal Area Monitoring (DAMOS) Program were utilized, as well as a newly developed tracer technique to track different lithologies of dredged material on the seafloor. Overall, the Portland Disposal Site Capping Demonstration Project showed that dredged material can be effectively placed, capped, and monitored at a deep water disposal site. Recommendations for improvements to the dredging and disposal operations, as well as to the monitoring methods, are provided for future project considerations.

Disposal and capping of dredged material is a management technique for the containment of sediments considered unsuitable for open-ocean disposal (unacceptably contaminated dredged material, or UDM) that has proven successful in Long Island Sound, in relatively shallow water (approximately $20 \mathrm{~m}$ ) and over a flat seafloor. Capping at deep water disposal sites ( $>40 \mathrm{~m}$ ) was an unproven management method due to a variety of factors, including historical difficulties in disposal barge positioning, and shortage of evidence confirming the formation of distinct UDM and capping layers. Refinement of dredged material management techniques and the implementation of the differential Global Positioning System (DGPS) during disposal and capping operations contributed to our ability to form, and monitor, discrete mounds in deeper water. This tightly controlled, closely monitored deep-water capping project has provided evidence that the technique can be successful in deeper waters.

In order to avoid any potential adverse environmental impact from such a demonstration, material dredged from the Royal River, Yarmouth, ME, deemed suitable for unconfined open-water disposal, was used as both "pseudo-UDM" as well as capping dredged material (CDM). The capping demonstration was designed to identify reaches (sections) of the Royal River project that were sufficiently distinct to permit identification of source materials after disposal. Finer grained sediment removed from the upper reaches of the river were designated as pseudo-UDM and placed as a discrete mound at PDS. Material from the lower reaches of the river, characterized by coarser grained material, was designated as the project CDM and was placed over the initial pseudo-UDM deposit as a cap. The capped disposal mound was formed within a basin feature on the PDS seafloor at a depth of $64 \mathrm{~m}$. After the completion of disposal and capping operations, the newly formed mound was surveyed and cored to confirm the existence of two distinct layers. This project design depended upon identifying characteristics of the reaches of the Royal River that could be analyzed in samples collected after disposal.

Based on the amount of dredged material disposed at the Royal River Project Area $\left(39,500 \mathrm{~m}^{3}\right.$ pseudo-UDM and 22,200 $\left.\mathrm{m}^{3} \mathrm{CDM}\right)$, the DAMOS Capping Model predicted 
the formation of a conical pseudo-UDM deposit approximately $1.2 \mathrm{~m}$ high with flanks extending up to $250 \mathrm{~m}$ from the central point of disposal, and a $20 \mathrm{~cm}$ cap. Although the volume of cap material was smaller than for normal projects (generally a minimum thickness of $50 \mathrm{~cm}$ ), the areal distribution of both pseudo-UDM and CDM observed in the demonstration, was relatively consistent with the model predictions.

An important component of the Portland Disposal Site Capping Demonstration Project was the identification of tracers within the Royal River that could be used to identify the sediment on the seafloor at the PDS. Prior to the excavation of sediment, 30 vibracores from three reaches (upper, middle, and outer) of the Royal River were collected and analyzed for a variety of potential tracers. Although no single tracer was identified that was both unique to one reach of the river and commonly observed in all collected samples, a statistical method of combining several biological and mineralogical parameters was found to be suitable for classifying the material types. The sediment fine fraction (63$500 \mu \mathrm{m})$ was selected as providing the most statistically robust data.

Monitoring at the Royal River Project Area in the southeast corner of the PDS utilized standard DAMOS techniques, including single-beam bathymetry, side-scan sonar, REMOTS $^{\circledR}$ sediment-profile images, as well as grab and core sampling. Results of the monitoring surveys showed that a discrete dredged material mound was detected on the seafloor within the Royal River Project Area. An accumulation of pseudo-UDM was detected to the south and southeast of the disposal buoy position, located in the relatively flat-bottomed basin targeted for disposal. Accurately detecting dredged material deposition in the surrounding area of more complex topography by single-beam bathymetry alone was complicated by survey artifacts. In this case, sediment-profile images and core data were key to mapping the footprint of both UDM and CDM on the seafloor.

The grab and core samples collected from the disposed dredged material were analyzed for the environmental tracers selected after analysis of Royal River Cores. The statistical tracer data were able to show a discernible difference between the CDM, pseudoUDM, and ambient material. The presence of historical dredged material at the project area complicated the analyses, as historical material shares biological characteristics with both native, ambient sediment (recolonization by benthic species, settling of planktonic species), and with recent dredged material (presence of freshwater species).

Statistical analyses showed that tracers successfully identified disposed dredged material layers collected from different regions of the estuary, but material from the middle reach had many overlapping characteristics that complicated the interpretation. The biological indicators were found to be more statistically robust than the mineralogical indicators. Differences in species composition of the microorganism populations corresponded to the contrasts among the brackish habitats of the three reaches of the Royal River. The statistical overlap of the pseudo-UDM and CDM samples collected in cores 
and grabs from the disposal mound was consistent with the sequence of disposal operations.

The results of the demonstration project provided recommendations for future cap monitoring projects in deep water disposal sites, including suggestions for modifications to both the monitoring protocols and to dredging and disposal operations. For areas of complex bottom topography, a higher resolution single-beam bathymetric survey grid (5 to 10-m lane spacing) or multibeam bathymetry is required to provide more precise depth information over a wider area of seafloor. For the demonstration project, the low volumes of dredged material, and the complications in the dredging and disposal schedule, contributed to uncertainty in the data interpretation. Operational complications that may occur with a larger project will have less of an impact, because larger volumes reduce the overall monitoring error.

Finally, the tracer technique that was selected demonstrated promising results in tracking dredged material at a subaqueous disposal site. Several recommendations were made to improve the method, including selecting tracers with the narrowest range in the dredging area, and sampling and analyzing the baseline (ambient and historical dredged) material prior to disposal of project material. 



\subsection{BACKGROUND}

\subsection{The New England Shipping Industry}

Over the past 300 years, the inhabitants of New England have relied on the resources of the North Atlantic for their livelihood, fostering the development of a rich maritime heritage. Coastal harbors, large and small, have supported various forms of commerce, transportation, and military activity since colonial times. These harbors provided sailing ships refuge from the ocean winds and waves, while allowing rapid access to open water. They also served as centers for trade, due to the constant exchange of raw materials and goods with fleets of transport ships. As a result, prosperous towns emerged from many of the small coastal communities clustered around the harbors of New England. Several of these towns developed into the major port cities of the Northeast (e.g., New Haven, CT; Boston, MA; Portland, ME).

The growth of the shipping industry was responsible for the expansion and urbanization of many New England harbor areas. Originally established to facilitate the transport of natural resources harvested from the New World to European markets, these ports have been forced to evolve with the changing global marketplace. Cargoes of lumber, livestock, and coal have now been replaced with electronics, automobiles, and petroleum. The wooden Clipper sailing ships were retired in the 1800 s, as larger steampowered vessels became more efficient at crossing the world's oceans.

Today, steel-hulled ships over 900 feet in length, powered by immense, diesel-fired engines and piloted with the use of satellites and computers, are utilized for movement of cargo and passengers across the oceans. In comparison to the earlier transport ships of the 1700 s, these larger and faster vessels readily carry a much larger volume of cargo (tonnage), and are capable of traversing the oceans in a fraction of the time. Large ships tend to be restricted, however, to ports that provide the navigational, anchorage, and dockage areas necessary to facilitate their deeper drafts and maneuverability requirements. As the average size of ocean-going cargo ships increased over the years, smaller and shallower ports were excluded from the resulting commerce and trade, causing economic decline. Ports that continued to be prosperous were successful in building larger docks and wharves, maintaining deeper channels, and removing hazards to navigation, to ensure safe conditions for large commercial and military vessels.

\subsection{The DAMOS Program}

The maintenance of safe, navigable waterways in areas of United States interest has been the primary responsibility of the U.S. Army Corps of Engineers (USACE) for the past 200 years. The maintenance or improvement of a port or harbor often requires some modification to the natural environment. Natural sedimentation processes such as soil and 
shore erosion tend to produce a shoaling effect by filling creeks, rivers, and bays with deposits of sand, silt, or clay. In order to create or maintain adequate depths for large transport ships and protect the economic viability of a port, some natural sediments must be mechanically removed, or dredged, from ship channels, anchorage areas, and docking facilities.

Dredging operations in New England waters typically involve the use of a clamshell bucket to extract rock, sand, gravel, mud, and clay from the bottom of waterways and transfer the materials to barges or on-shore facilities for disposal (Figure 1-1). A number of disposal alternatives exist for dredged material, but the majority of these materials are transported to open water and deposited at predefined dredged material disposal sites. To efficiently manage the large volumes of dredged sediments, the New England District (NAE) of the U.S. Army Corps of Engineers has taken a broad, programmatic approach to dredged material management.

The dredged material management process is a multi-step decision process incorporating project evaluation, disposal compliance inspections, monitoring, mitigation, and enforcement. The Disposal Area Monitoring System (DAMOS) Program is a critical part of this process, concerned with the long-term monitoring of open water dredged material disposal sites. Established in 1977, the DAMOS Program investigates and minimizes any adverse physical, biological, or chemical impacts of dredging or dredged material disposal. The cooperative efforts of NAE, scientists, and ocean engineers have resulted in the development and implementation of a flexible, tiered management approach used to achieve the goals of the DAMOS Program.

Before dredging operations begin, the proposed project sediments are sampled and tested to determine their physical and chemical properties. Sediments originating from most of coastal New England are found to have low to undetectable contaminant levels and are considered suitable for unconfined open water disposal. This material can be readily deposited into subaqueous disposal sites or used for a number of beneficial projects (i.e., beach nourishment, marsh and island creation, landfill for development projects). However, since the 1978/79 disposal season, the value of these sediments as capping dredged material (CDM) has been fully realized by the DAMOS Program (Fredette 1994).

\subsection{Subaqueous Capping}

Maintenance or improvement dredging operations conducted in urbanized or industrialized regions tend to yield sediments that contain a variety of environmental contaminants associated with anthropogenic activity. Sediment deposits dredged from industrialized coastlines may contain chemicals that have a potential for adverse environmental impact (i.e., heavy metals, PCBs, and other organic compounds). If found in sufficient 
concentrations, this material is classified as unacceptably contaminated dredged material (UDM), requiring special handling, storage, and disposal techniques (Fredette 1994).

One cost-effective and environmentally sound alternative for large scale dredging projects is to dispose of the UDM at open water disposal sites monitored by the DAMOS Program, and cover the initial deposit with a larger volume of CDM. Capping is a subaqueous containment method that uses CDM to overlay and completely cover a UDM deposit, isolating the contaminants from the marine environment (SAIC 1995). Capping was first introduced as a management technique of the DAMOS Program during the 1978/79 disposal season with the development of the Stamford-New Haven mounds (STNH-N and STNH-S) at the Central Long Island Sound Disposal Site (CLIS; SAIC 1995). Over the past 18 years, monitoring and research activities within the DAMOS Program regarding subaqueous capping of UDM have evolved, resulting in significant progress in pre-project planning and the ongoing development of management strategies.

A successful capping project depends on the selection of an appropriate disposal site, identification and access to large volumes of CDM, as well as the careful control of dredging and disposal operations. Currently, the DAMOS Program maintains ten closely monitored open water disposal sites along coastal New England capable of receiving both clean and contaminated sediments (Figure 1-2). However, the low kinetic energy environment, shallow to moderate water depths $(20 \mathrm{~m}$ to $22 \mathrm{~m})$, and gently sloping, regular bottom topography have made CLIS the preferred proving ground for innovative dredged material management and disposal techniques.

Capping operations on a flat or gently sloping bottom usually require a $2: 1$ to $6: 1$ CDM to UDM ratio to adequately cap a contaminated sediment deposit. An exception was during the 1993/94 disposal season at CLIS, when a capped mound composed of over $1,100,000 \mathrm{~m}^{3}$ of sediment dredged from the federal channel and active ports in New Haven Harbor was constructed on the seafloor (Morris et al. 1996). The material was placed in the center of an artificial containment cell formed by the controlled deposition of small to moderate volumes of dredged material over a ten-year period. The resulting bottom feature was found to be a broad, stable confined aquatic disposal (CAD) mound with a CDM to UDM ratio of $0.96: 1.0$ (Morris and Tufts 1997). The artificial containment measures restricted the lateral spread of the large, strategically placed UDM deposit $\left(590,000 \mathrm{~m}^{3}\right)$, requiring less $\operatorname{CDM}\left(569,000 \mathrm{~m}^{3}\right)$ to completely isolate the contaminated material.

Natural containment measures (i.e., basins, bedrock outcrops, terminal moraine deposits, etc.) also can be employed in the development of capped mounds. The preexisting glacial features of Massachusetts Bay Disposal Site (MBDS), Cape Arundel Disposal Site (CADS), Portland Disposal Site (PDS), and Rockland Disposal Site (RDS) lend themselves for use in subaqueous capping operations. Although significantly deeper 


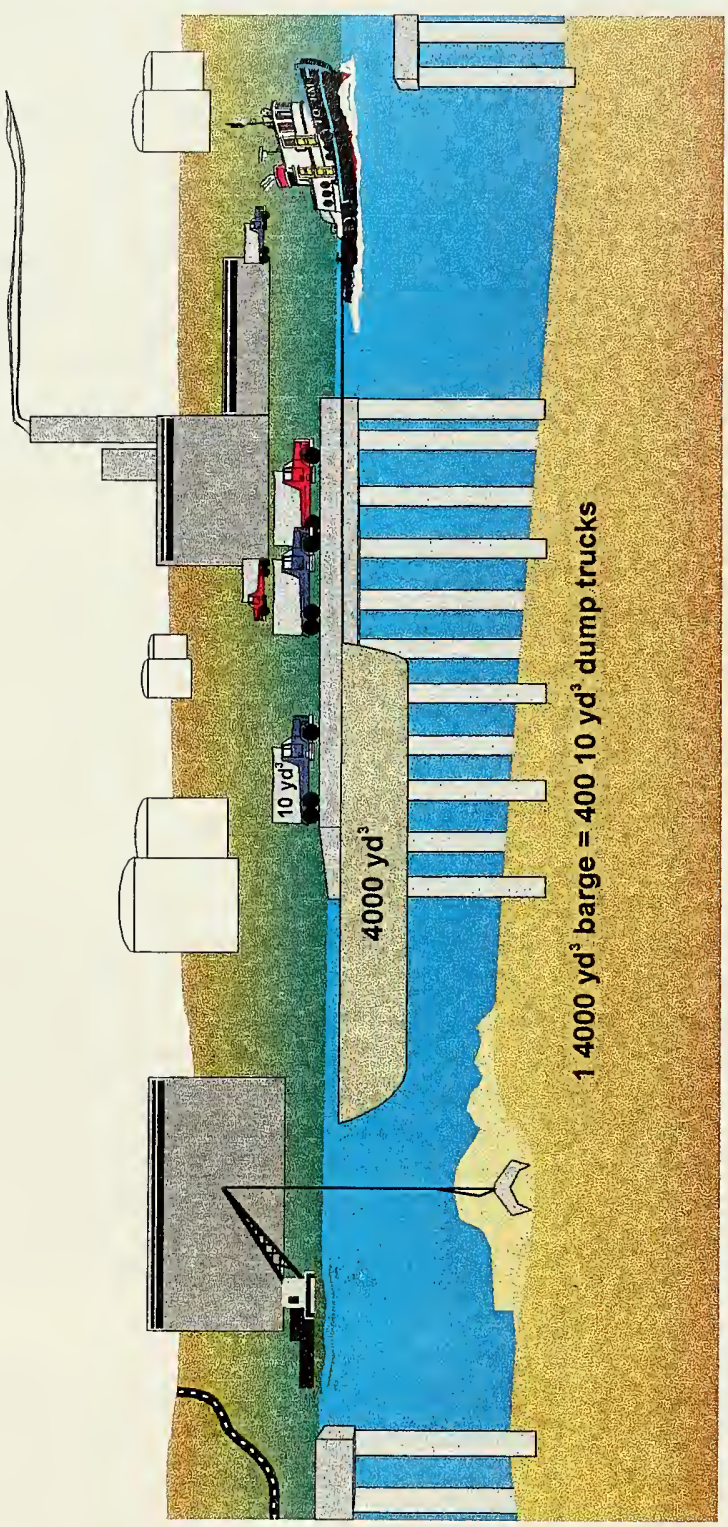

.

$\therefore$

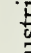


than CLIS, the successful development of capped disposal mounds within the boundaries of MBDS, CADS, PDS, and RDS appears feasible (Morris 1996). In fact, capping operations were conducted at PDS in 1992 and at the historic Boston Foul Ground (BFG) in 1983 (Wiley 1996). However, the limited data collected from these projects did not provide sufficient assessment to implement subaqueous capping as a deep-water disposal technique.

In the past, disposal and capping operations at deep water disposal sites ( $>40 \mathrm{~m}$ ) were complicated due to difficulties in disposal barge positioning, yielding a wider dispersal pattern than anticipated, and the lack of a discrete UDM deposit (Wiley 1995). In addition, concerns over the dissipation of fine-grained sediments in the water column and shortage of evidence confirming the formation of two distinct disposal mound layers (CDM over UDM) were obstacles to continued use of this management strategy (Dolin and Pederson 1991). Refinement of dredged material management techniques and the implementation of the differential Global Positioning System (DGPS) during disposal and capping operations improved discrete mound development in deeper water. But only a tightly controlled, closely monitored deep water capping project will provide insight to the behavior of material on the seafloor.

\subsection{Deep-Water Capping at the Portland Disposal Site}

The Portland Disposal Site Capping Demonstration Project was initiated due to renewed interest in subaqueous capping at the deep water PDS. As the northernmost icefree port on the eastern coast of North America, Portland Harbor is essential for the survival and prosperity of northern Maine and the Canadian provinces of New Brunswick and Quebec during the winter months. Over the years, this port has grown to support the expanding industry and international trade, which now financially sustains the majority of the Portland metropolitan area. As the largest port in the state of Maine, a total of 14 major marine terminals have been established on the banks of the Fore River (Custom 1995; Figure 1-3).

Initial projections indicated that over $765,000 \mathrm{~m}^{3}$ of material will be excavated from the bottom of Portland Harbor during the next federal dredging project. Although all but a small portion of the federal material from the harbor has been classified as suitable for unconfined open-water disposal, capping at deep-water sites such as PDS will still potentially benefit future projects. The remaining small volume of material will not be dredged. The management study for the project has included evaluation of the feasibility of subaqueous capping of the Portland Harbor sediments at an open-water disposal site as a valid, cost-effective, and environmentally sound disposal method.

The Portland Disposal Site lies approximately $13.16 \mathrm{~km}$ east of Dyer Point, Cape Elizabeth, Maine (Figure 1-4). This $3.42 \mathrm{~km}^{2}$ DAMOS disposal site, centered at $43^{\circ} 34.100^{\prime} \mathrm{N}, 70^{\circ} 02.000^{\prime} \mathrm{W}$, is characterized by rough, irregular bottom topography 


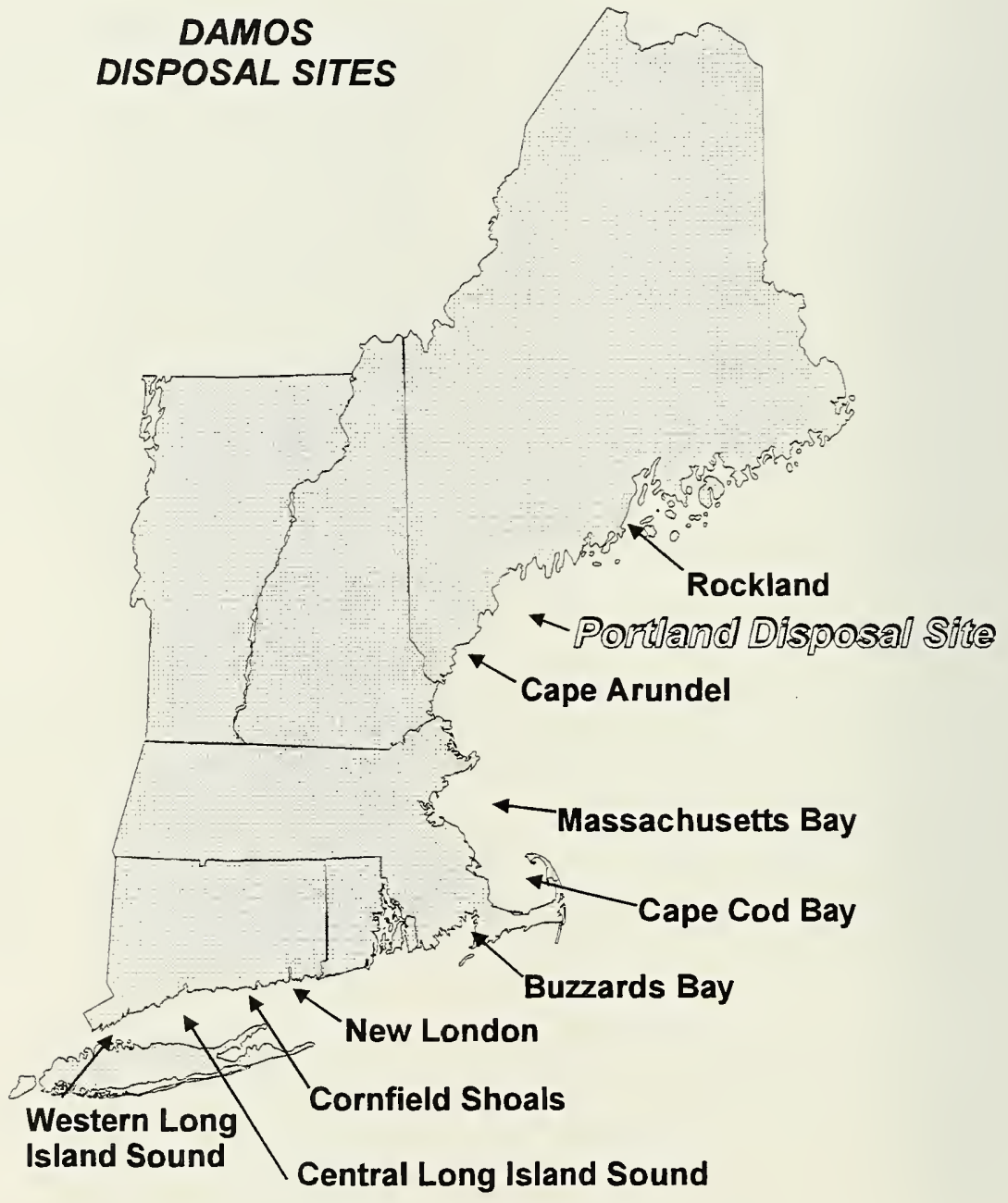

Figure 1-2. Geographic locations of the ten DAMOS disposal sites along the coast of New England 
with water depths that range from $42 \mathrm{~m}$ to $74 \mathrm{~m}$. The regulated and monitored deposition of dredged material has been occurring at PDS since 1977, with an average annual disposal volume of approximately $99,000 \mathrm{~m}^{3}$ (Morris 1996). However, usage of this region as a disposal site dates back to 1947 , as material was disposed over a $17.7 \mathrm{~km}^{2}$ irregularly shaped area of seafloor surrounding the current PDS boundaries (Figure 1-4).

The depositional environment of PDS, especially within the deeper fine-grained basins, indicates that volumes of dredged material can be placed without movement of these deposited sediments beyond the disposal site boundaries. The demonstration project was planned to take advantage of the irregular topography of the PDS. Strategic placement of a disposal buoy within the center of a basin surrounded by natural ridges would serve to contain the initial UDM deposit within the confines of the basin. The basin features would minimize any lateral spread of the UDM deposit, and aid in the complete and efficient isolation of the project material with a similar volume of CDM.

A capping project conducted at PDS from October 1991 through July 1992 set a precedent, as all other DAMOS sediment capping operations were conducted at disposal sites with water depths of approximately $20 \mathrm{~m}$. The 1991-92 capping project consisted of a $13,300 \mathrm{~m}^{3}$ UDM deposit of silt and clay, capped with $37,800 \mathrm{~m}^{3}$ of CDM consisting of sand and silt (Wiley 1996). Comprehensive analysis of the sediments collected over the surface of the capped mound showed chemical concentrations corresponding to the levels detected in the CDM before dredging operations commenced. However, analysis of sediment-profile photographs revealed a heterogeneous mixture of sand and silt components in the project capping material which confounded the physical differentiation between the UDM and CDM layers. As a result, insufficient data were gathered during the 1991 and 1992 monitoring cruises to unequivocally determine the behavior of the dredged material at deeper sites, and the ability to form distinct UDM and CDM layers without mixing (Wiley 1996).

A joint effort between NAE, EPA, and the Casco Bay Program formulated a closely monitored capping demonstration project at PDS to gather more data on the behavior of dredged material at deeper water containment disposal sites. A small dredging project (estimated barge volume of $86,000 \mathrm{~m}^{3}$ ) from the Royal River in Yarmouth was used to examine the feasibility of capping at PDS. The Royal River project was selected due to the distinctive sediment characteristics within the estuary, and the presence of sediments deemed suitable for unconfined open water disposal. 


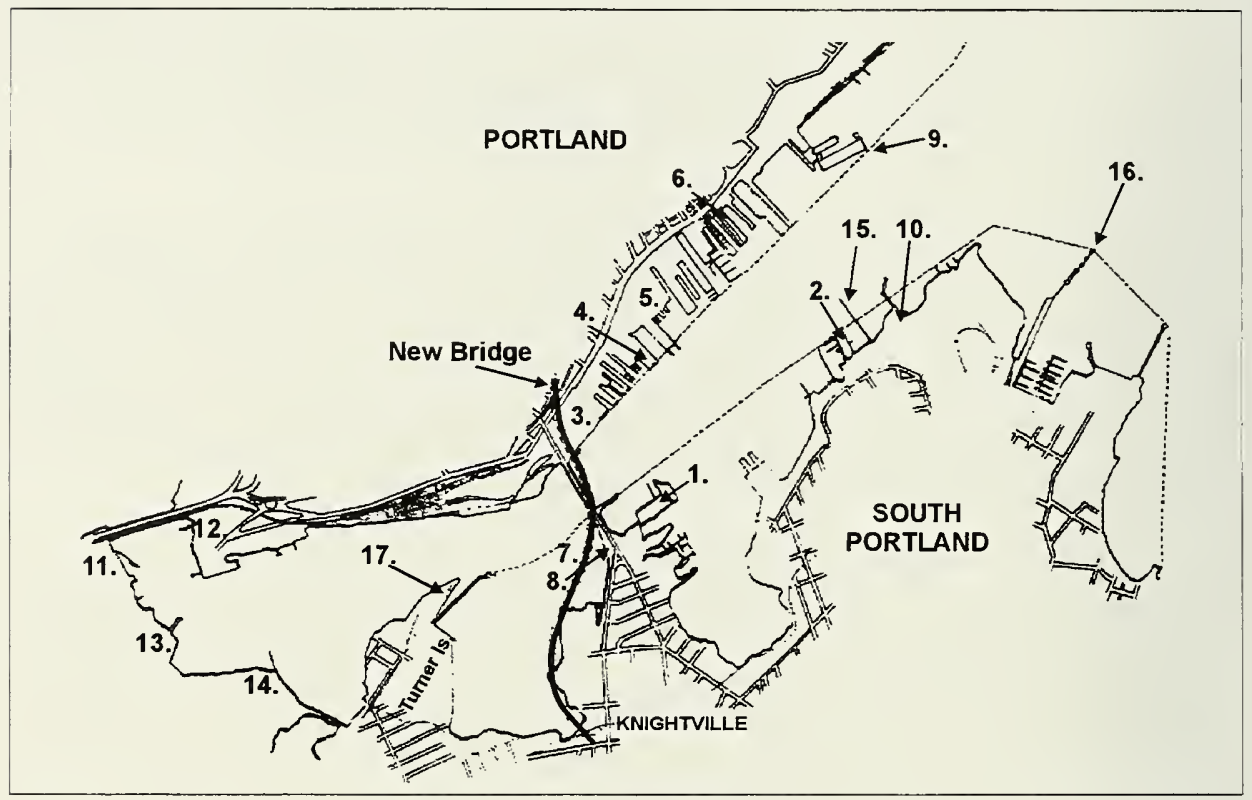

\section{Power Company}

2. South Portland Shipyard/Marina

3. Portland International Terminal Co. (Intl. Ferry)

4. Portland Terminal Co. (Wright Wharf)

5. Portland Fish Pier

6. Marine State Pier

7. NPDES Major Discharge

8. Water Pollution Control Plant

9. Bath Iron Works
10. Gulf Oil Terminal

11. Getty Terminal

12. Merrils Marine Terminal

13. Mobil Oil Terminal

14. Northeast Petroleum

15. Portland Pipeline Pier 1

16. Portland Pipeline Pier 2

17. Star Enterprise Terminal

Figure 1-3. Geographic location of major marine terminals and public works facilities associated with urbanization in Portland Harbor (Custom Communications 1995) 

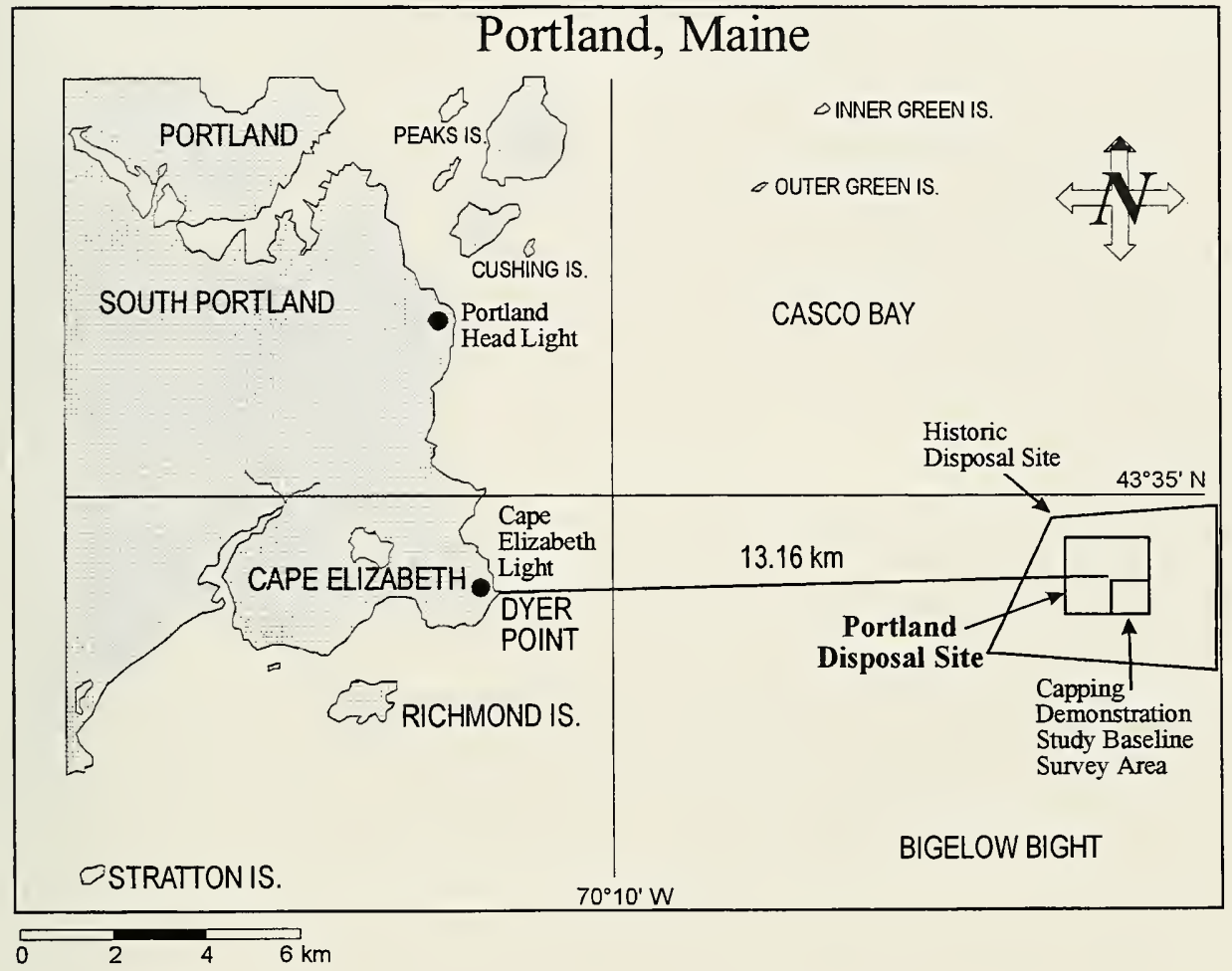

Figure 1-4. Location of the current and historic boundaries of Portland Disposal Site, as well as shore station benchmarks 
This page left blank intentionally 


\subsection{THE CAPPING DEMONSTRATION PROJECT}

\subsection{Dredging of the Royal River}

The Royal River is one of the many tributaries along the rocky, irregular coast of Maine providing drainage of rain and melt waters from the foothills of the Appalachian Mountains. Chandler and Toddy Brooks as well as a number of smaller creeks and streams empty into the upper reaches of the Royal River, allowing the transport of freshwater run-off and an abundance of eroded soils downstream (Figure 2-1). The Royal River encounters the influence of tides from the Gulf of Maine and Casco Bay just below the lower falls at Yarmouth, ME. Once over the falls, the sediment-laden freshwater is mixed with seawater intruding from Casco Bay, establishing the upper reaches of the Royal River estuary.

The Royal River converges with the smaller Cousins River at Browns Point, Yarmouth, ME (Figure 2-1). Tidal effects, in conjunction with the combined sediment loads from the two river systems, cause natural deposition of silts and clays within tidal flats along the banks of rivers and the development of a complex constructive delta within the western portions of Casco Bay (Figure 2-2; MSPO 1983). The natural processes within the river associated with periods of increased freshwater run-off (spring melt) and higher current velocities during ebbing tides maintain narrow channels through the riverbed and delta. However, these naturally maintained tidal channels tend to be irregular in shape and depth, as well as to follow the meanders of the respective river basins. To preserve the uniform navigational channels required by most vessels, dredging of excess sediment is required.

Currently, the Royal River is used for recreational activities, providing protection and rapid access to open water for a moderate number of smaller vessels ( 40 feet in length). However, since the establishment of the first settlement in 1635, the waters of Royal River have served as the source of prosperity for the people of Yarmouth, ME (Attanas and Hinkley 1997). The river provided the colonists with drinking water, food, power generation, and transportation. Occasionally displaced by floods or wars with the native Americans and French, the people of Yarmouth, ME, would always return and rebuild on the river banks.

During the industrial revolution, as many as 60 saw, grist, textile, and paper mills were established along the course of Royal River. Timber, produced by extensive logging activities in North Yarmouth, was transported on the waters of the river to the awaiting saw mills. The excess wood and waste products of the paper mills were sent down stream, flowing out into Casco Bay. 


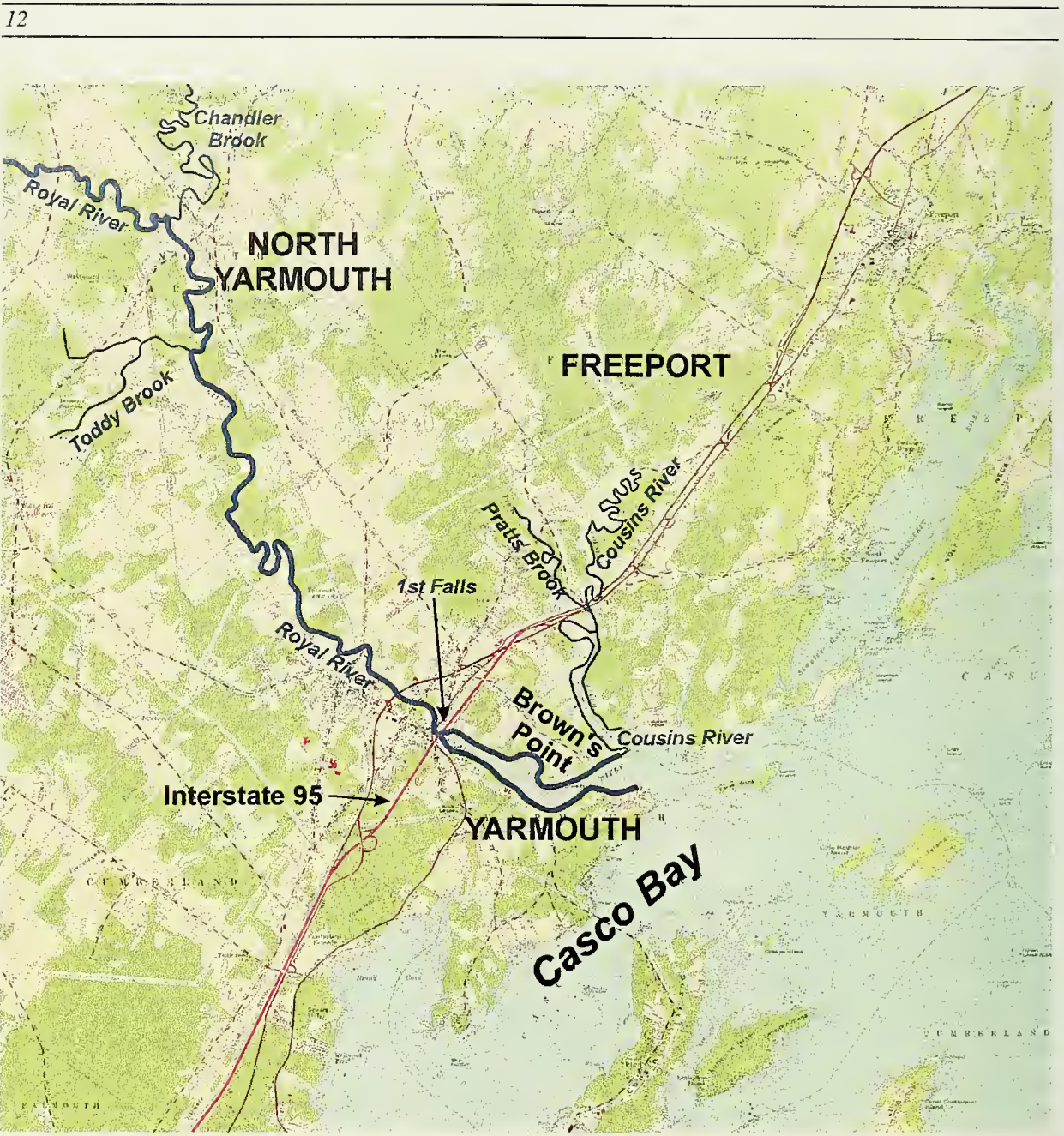

Figure 2-1. Topographic map of a portion of Cumberland County, ME and the Royal River watershed area 


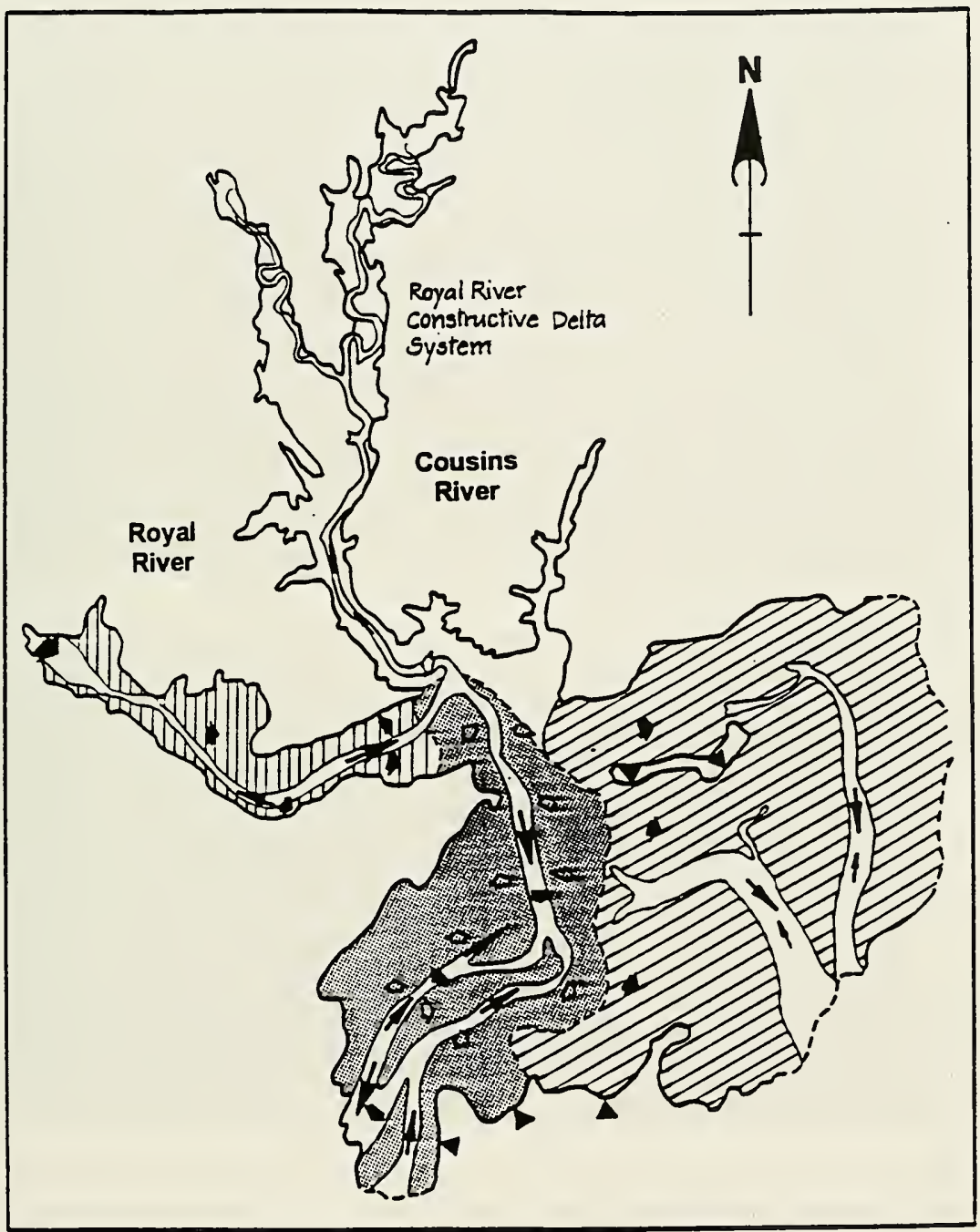

Figure 2-2. Process map of the Royal and Cousin river systems (MSPO 1983). Shaded area is constructional area at the confluence of the two rivers. Vertical lines around the Royal River indicate tidally-influenced depositional/erosional processes. Long narrow arrows show water flow; short broad arrows show sediment transport. 
The largest consumer of the processed lumber was the ship building industry occupying much of the shoreline below the first falls. Large, two- and three-masted sailing ships were painstakingly assembled and launched from the banks of the river during most of the 19th century (Figure 2-3A). The USACE excavated the first navigation channel in the 1890 s to provide sufficient maneuverability for the large sailing ships built at these boat works. In addition, the larger, coal-fired steam ships carrying raw materials and goods from the town of Yarmouth to market in Portland or abroad required passage. Over time, the increasing demand for larger ships and the development and widespread use of railroads along the coast of Maine resulted in the decline and collapse of the ship building industry in Royal River.

The Great Depression, business competition, and diminishing dependence on the water resources eventually led to the decline of industry along the banks of the Royal River. The estuary provided a subsistence level shell fishery for the people of Yarmouth. A thriving fish cannery replaced the boat works on the banks of the river. From 1910 to 1979 , tons of sardines and other fin fish harvested from Casco Bay and the Gulf of Maine were brought upriver by trawlers and skiffs on a daily basis. With only one factory remaining, the working Yarmouth Harbor of the 1800 s was replaced by a quaint town landing used more for recreation than commerce (Figure 2-3B).

Although the waters of Royal River no longer carried timber and the by-products of industry downstream, the river did continue to transport sediments from its watershed area. Due to the depositional environment within the river, normal sedimentation processes would incorporate this sand, silt, and clay into deposits along the banks and at the mouth of the river, eventually filling in the channel dredged in 1890. Maintenance dredging of the federal channel occurred several times between 1890 and 1960 to facilitate the movement of fishing boats and their catches upriver to the cannery.

As of the 1990s, sediment had partially infilled the Royal River once again. The river was becoming too shallow to support draft requirements of the recreational fleet using the marinas and anchorage area in Yarmouth Harbor. As a result, the USACE planned a maintenance dredging project to remove the excess sediment from the navigational channel and anchorage area for the fall of 1995.

Waterways scheduled for maintenance or improvement dredging are surveyed to establish accurate depths (bathymetry), and the sediments to be excavated are sampled and analyzed. The bathymetric survey of an area allows for the calculation of "in-place" volumes of material to be removed from a riverbed in order to achieve a specified channel depth. Sediments are collected to characterize the physical and chemical nature of the various layers of material for dredged material classification (UDM versus CDM). If the bottom is composed of mud or sand, a series of cores are usually collected to provide a deep cross section of the subject sediments. 

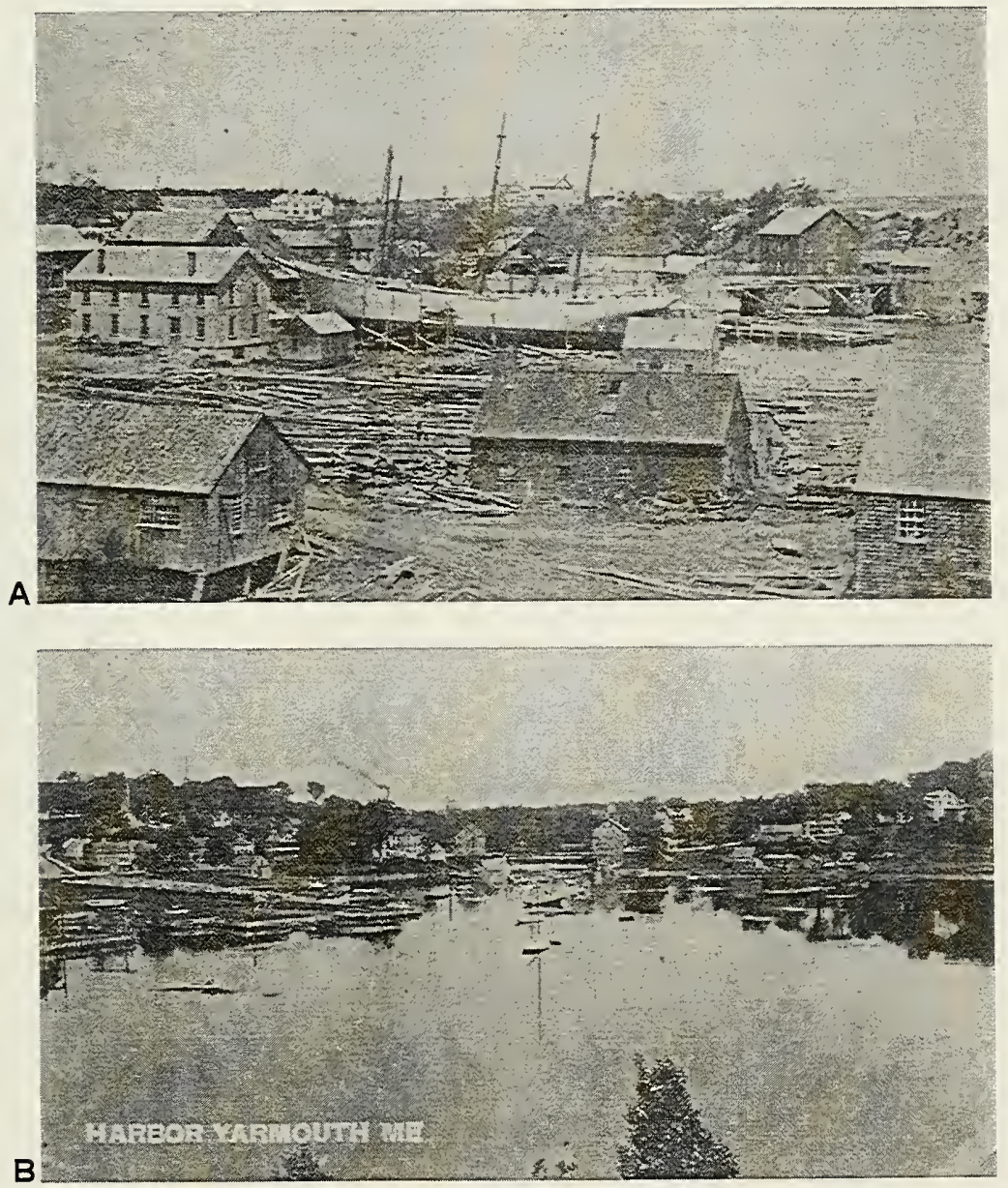

Figure 2-3. Photographs at Yarmouth Harbor:

A. Ship under construction, west bank, circa 1875

B. Status of the harbor circa 1915 (Attanas and Hinkley 1997) 
The USACE performed a preliminary pre-dredging survey from the base of the falls to the mouth of the Royal River consisting of a bathymetric survey and the collection of 12 sediment characterization cores (A1 through E3; Figure 2-4). Analysis of bathymetric data yielded sediment volume estimates of $73,000 \mathrm{~m}^{3}$ to be removed from the channel and anchorage area, in order to provide safe navigational depths at low tide (Figure 2-5). Testing of the sediments collected in Cores A-1 through E-3 indicated that all the material to be dredged from the river basin was suitable for unconfined open water disposal or for beneficial use projects and classified as CDM.

This moderate volume of high quality sediment was ideal for the capping demonstration project at PDS. The Royal River dredged material for the Portland Disposal Site Capping Demonstration Project presented no environmental risk. Because the sediments throughout the riverbed were determined to be suitable for open water disposal, the inability to successfully construct a capped mound consisting of two distinct layers would have no adverse environmental or ecological impact. Initial baseline studies in support of the capping demonstration project began in August 1995, and monitoring continued through February 1997, as the capped mound was formed within a basin feature on the PDS seafloor at a depth of $64 \mathrm{~m}$ (Figure 2-6). Because of the complexity of the timing of dredging and monitoring operations, the details of the time line presented in Figure 2-6 are provided in Section 2.2.

\subsection{Capping Demonstration Project Time Line}

\subsubsection{Baseline Surveys at the Royal River and Royal River Project Area}

In August of 1995, SAIC collected 30 vibracores (RR-1 through RR-30) from three reaches (upper, middle, and outer) within the Royal River navigational channel to supplement data acquired from 12 cores collected by NAE (Figure 2-4). The cores provided deep cross sections (up to $3 \mathrm{~m}$ ) of the riverbed and allowed for the identification of several tracers within the project sediments capable of tracking dredged material from its origin in the river, to the disposal mound on the PDS seafloor.

Detailed analysis of 11 of the 30 sediment cores indicated both microfossils and mineralogical components in the sediment could be used to identify source material removed from the upper and outer reaches of the river (Figure 2-4). The most promising technique was the determination of assemblages of two informal grains of unicellular, eukaryotic microorganisms (Foraminifera and Thecamoebina). These organisms form hard shells, which may be preserved during the natural accumulation of sediment in the river. As a result, these shells can be examined in the dredged material and used to recognize the environment of the original deposition. Differences in species composition of the microorganism populations would correspond to the contrasts between the freshwater 


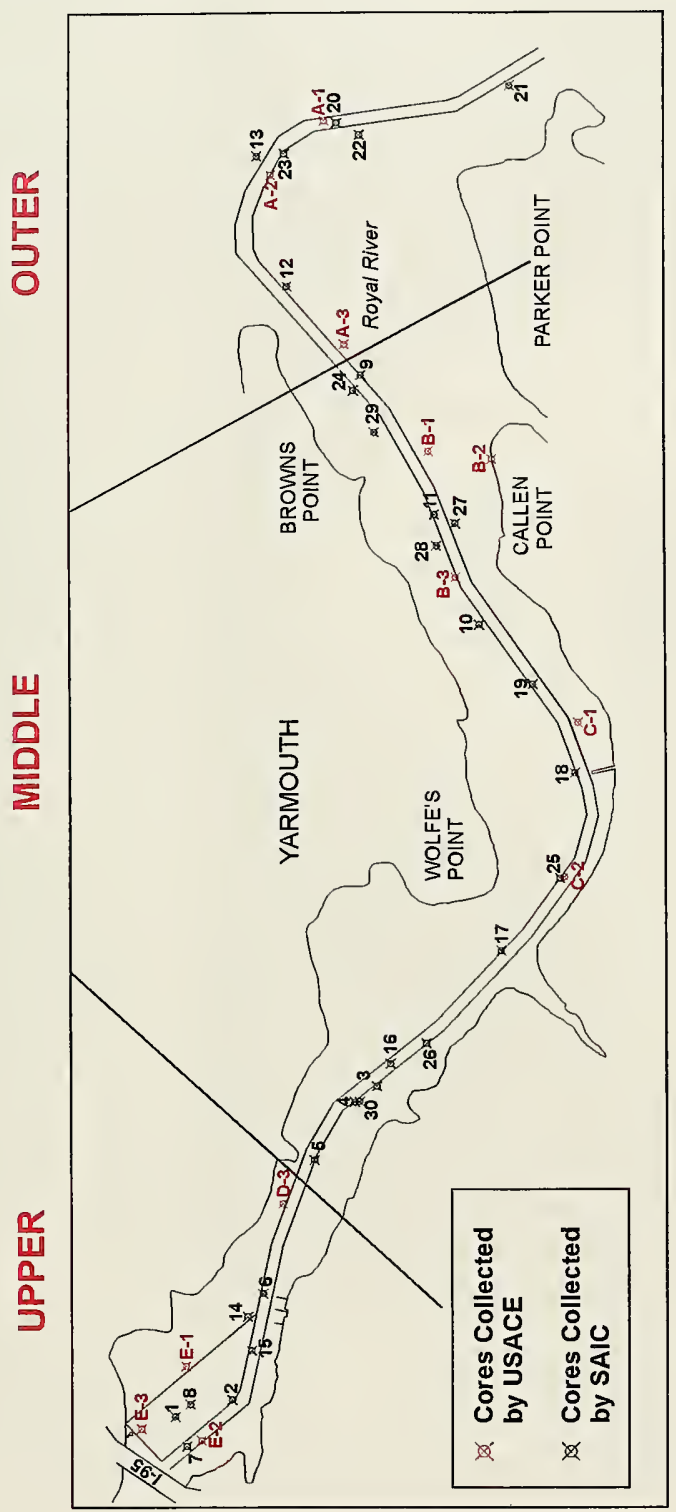

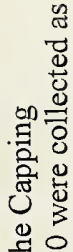

욜

눈

总总贾

要的

\% 당

它句

舟导

능

엉흥

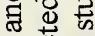

몽유

记

寻 步

डु

응. 秃

空

. 00

¿ 8

on

罗颔

ว

क्ष

印苛

$\sum 00$

芯

卷焉

$\Rightarrow$ 등

毛它

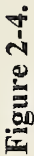



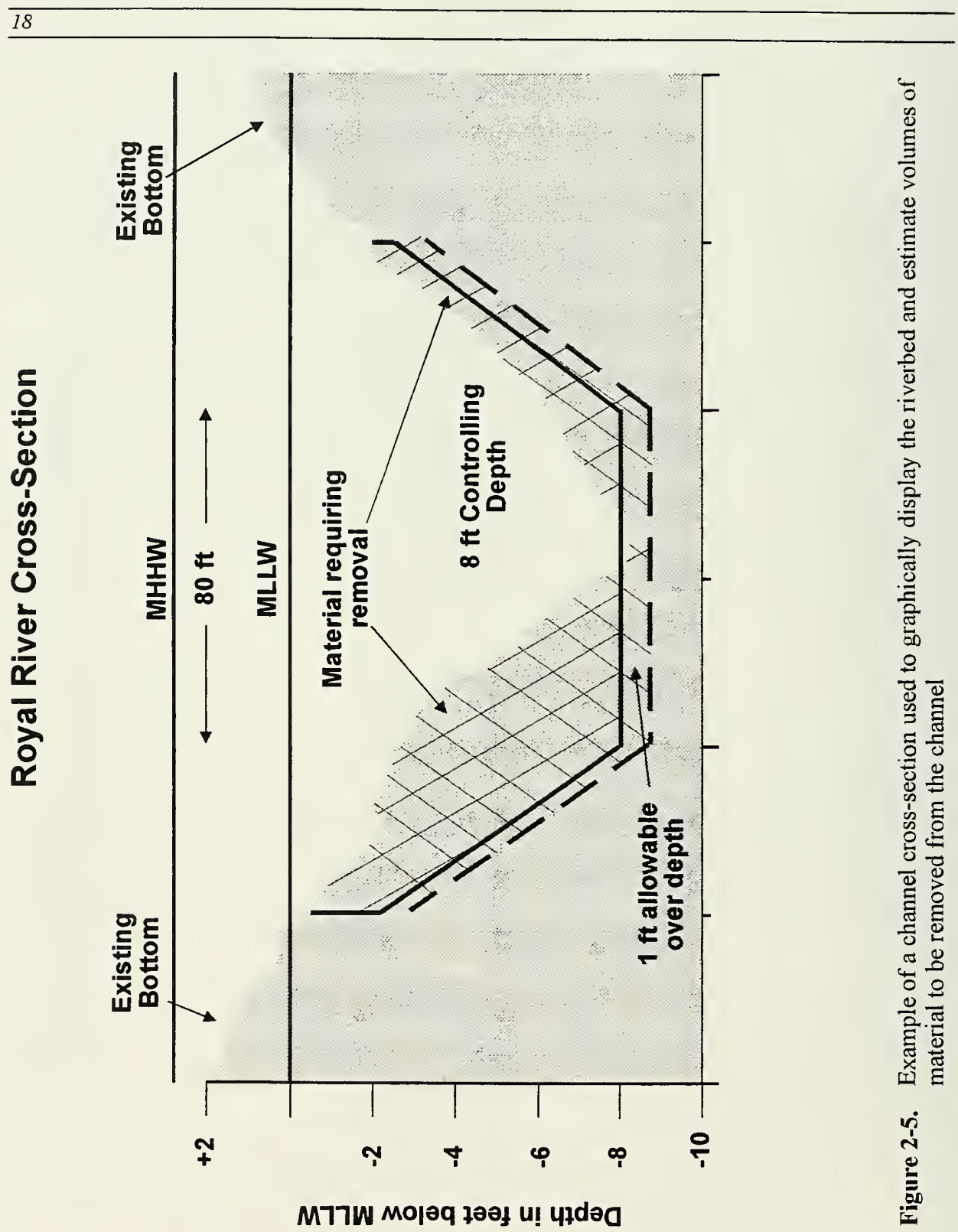
habitat of the upper river zone versus the brackish and saltwater environments of the middle and outer zones (Figure 2-7).

Also in the summer of 1995, SAIC and NAE selected an $800 \mathrm{~m} \times 800 \mathrm{~m}$ area in the southeast corner of PDS as the Royal River Project Area (Figure 2-8). The southeast quadrant of the disposal site was selected due to limited historic disposal activity and availability of basin features that would act as natural containment measures to restrict the lateral spread of the dredged material mound. SAIC completed a baseline bathymetric survey over the $0.64 \mathrm{~km}^{2}$ PDS project area in August 1995 for comparison with all future project survey results.

\subsubsection{Dredging Operations, Fall-Winter 1995-96}

In late October 1995, the taut-wired disposal buoy "PDA" was deployed at $43^{\circ} 33.790^{\circ} \mathrm{N}, 70^{\circ} 01.514^{\prime} \mathrm{W}$, at the center of a small basin within the Royal River Project Area at PDS (Figure 2-8). Dredging operations in the Royal River were scheduled to commence in mid-November 1995 with a target completion date of late December. Operational difficulties with the contractor's dredging equipment, however, caused significant delay in the initiation of material excavation, as well as slow progress once dredging was initiated (Figure 2-6). By late December 1995, no dredged material had left the Royal River for disposal at PDS. The delays in the Royal River dredging operations began complicating other projects utilizing PDS for disposal.

In South Freeport, ME, a small improvement dredging project at a local marina in the Harraseeket River was scheduled to provide additional CDM for the completed Royal River pseudo-UDM deposit. If dredging operations in the Royal and Harraseeket Rivers were completed in the correct sequence, the marina project would have produced a total of $10,000 \mathrm{~m}^{3}$ of capping material to supplement the volume of available CDM from Royal River. Approximately $2,800 \mathrm{~m}^{3}$ of sediment dredged from the Harraseeket River was deposited to the north and west of the PDA 95 buoy position between December 19, 1995, and January 5, 1996, introducing fresh dredged material into the project area before any material was dredged from the Royal River. When this complication was detected by NAE in early January 1996, the remaining $7200 \mathrm{~m}^{3}$ of material was redirected to the U.S. Coast Guard Class A disposal buoy ("DG"; Figure 2-8).

By January, the first barge loads of material had left the Royal River for disposal at PDS, but was directed to the DG buoy in an attempt to streamline the dredging and disposal operations before Royal River sediments were deposited within the project area. From January 6 through February 15, a total estimated barge volume of $22,000 \mathrm{~m}^{3}$ of Royal River material was deposited at the DG buoy. After mid-February, however, no further progress was made by the dredging contractor. The Royal River dredging project 


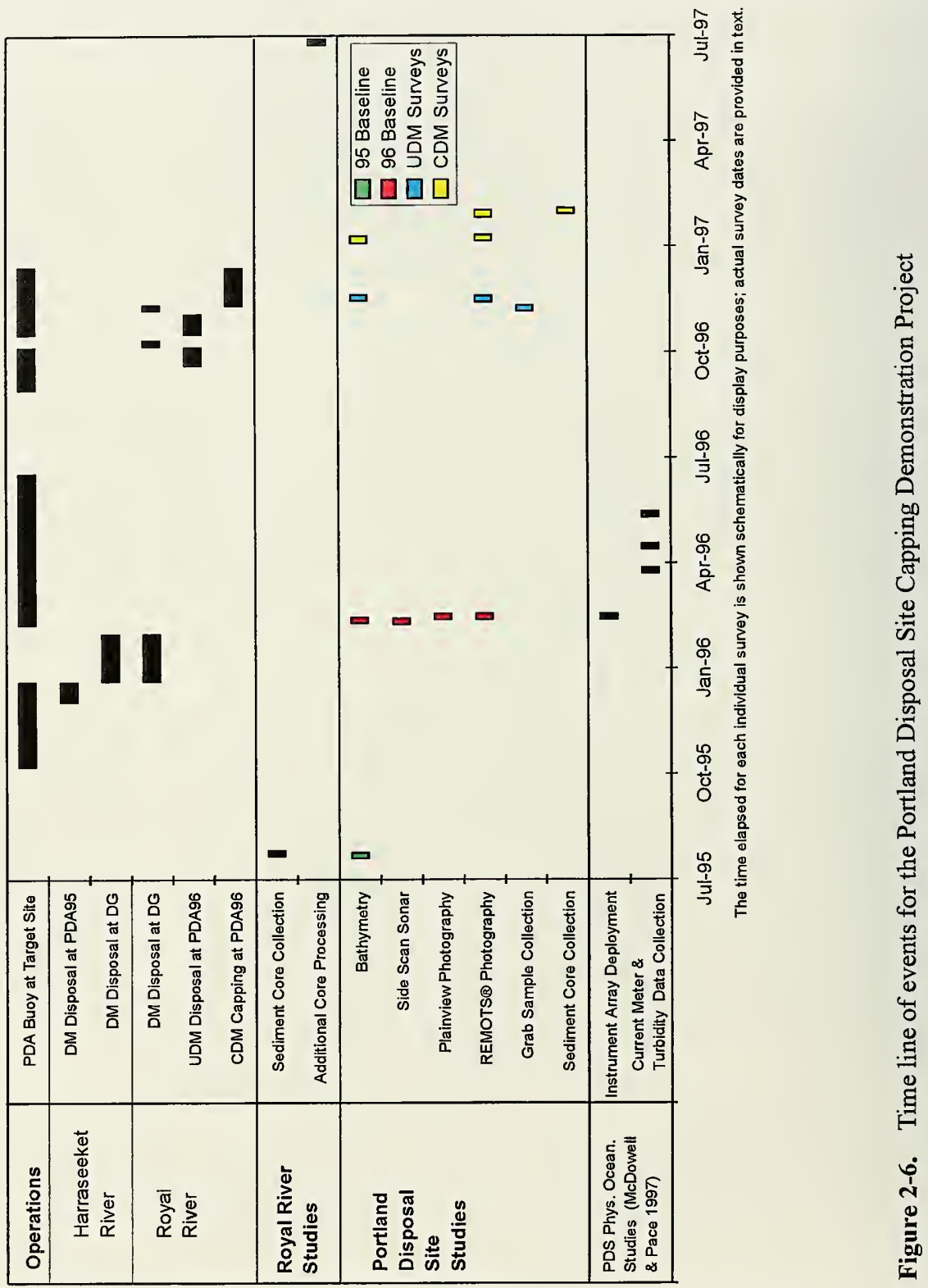



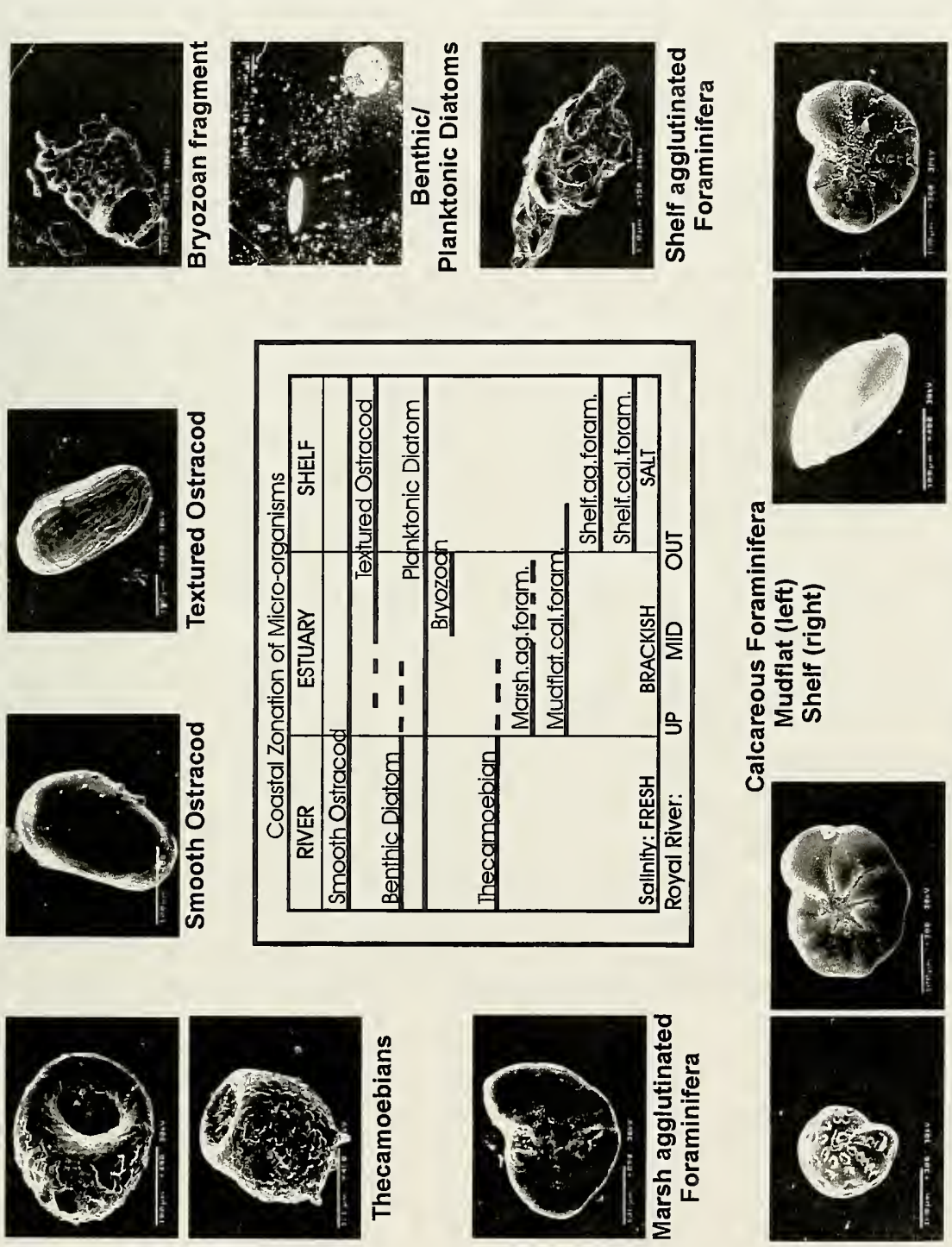

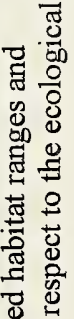

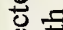

定

额

을 급

됴

్ㅗㄹ ฮै

\&

屯. 옹

定哭

तह के

各焉

녕

兵 芌

틀 을

304

귬

으임을

苍

욜 등

गे

$\Phi$

क

แ

宩

를

要

듸 긍

ำ 


\section{Portland Disposal Site December 1977 Bathymetry}

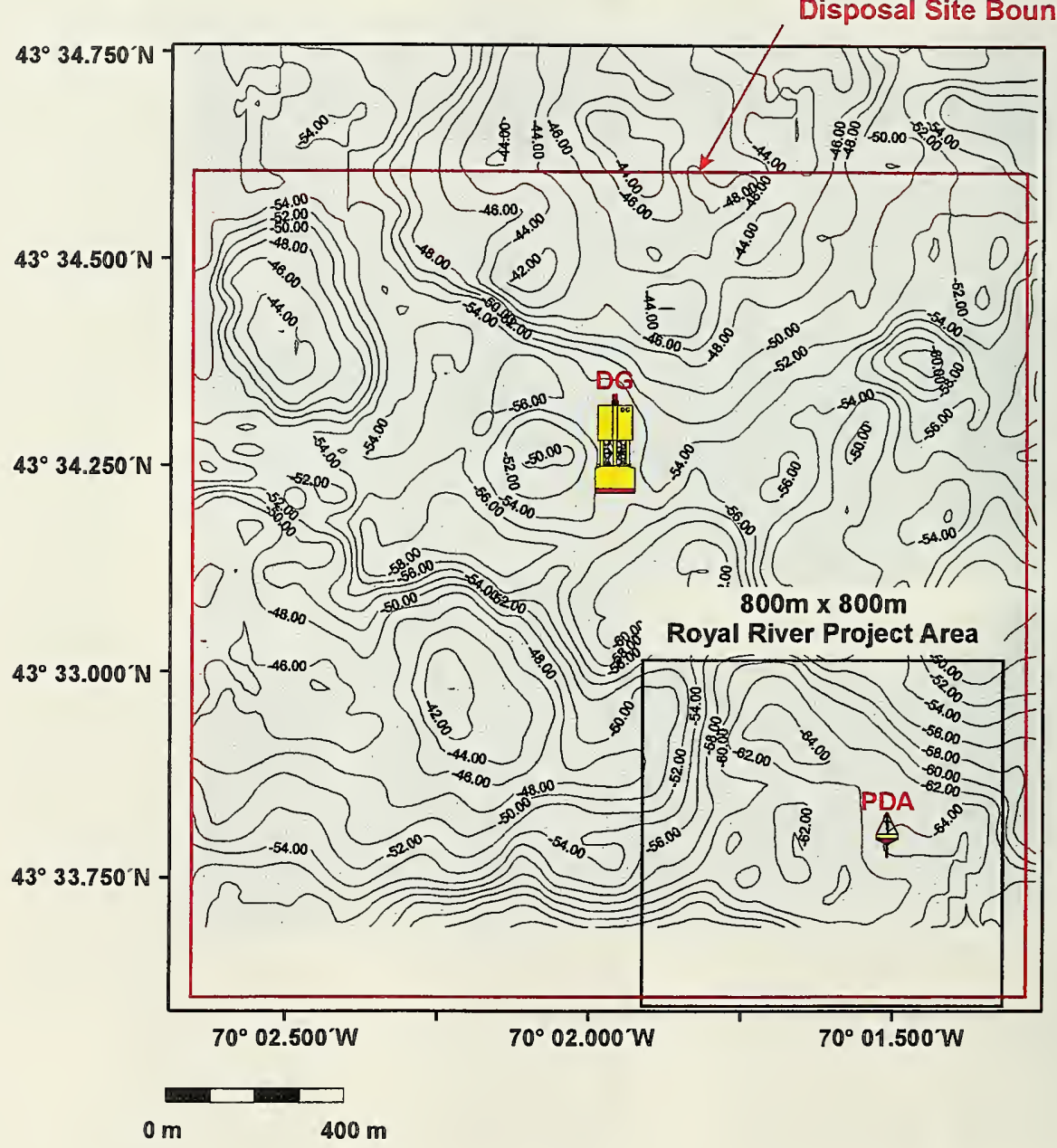

Figure 2-8. Bathymetric chart of the $1950 \mathrm{~m} \times 1875 \mathrm{~m}$ master survey (NAD 27) performed over PDS in December 1977, $2.0 \mathrm{~m}$ contour interval (MLLW) 
was discontinued until a new company could be selected to complete the task. The PDA buoy holding station over the Royal River Project Area at the disposal site was withdrawn.

\subsubsection{Expanded Royal River Project Area Baseline Survey}

In late February 1996, NAE determined that a new baseline survey was required due to the disposal of the Harraseeket River material at the Royal River Project Area at the PDA buoy. SAIC conducted a second bathymetric survey, in conjunction with side-scan sonar imaging of the bottom, over an expanded $1950 \mathrm{~m} \times 1000 \mathrm{~m}$ survey area. The February 1996 survey occupied a larger $1.95 \mathrm{~km}^{2}$ area over the southern region of PDS in an effort to better characterize the disposal site seafloor and aid in the placement of a bottom-mounted instrument array deployed to collect physical oceanographic data throughout the winter and spring of 1996 (McDowell and Pace 1997). In addition, a 24station, Remote Ecological Monitoring of the Seafloor (REMOTS ${ }^{\circledR}$ ) sediment-profile photography survey was performed over the $800 \mathrm{~m} \times 800 \mathrm{~m}$ project area, obtaining information on the composition and distribution of the Harraseeket River dredged material and ambient sediments at the PDA buoy position (Section 4.3).

\subsubsection{Pseudo-UDM Dredging Operations, Fall 1996}

The contract for the completion of the Royal River dredging project was awarded to Prock Marine of Rockland, ME. SAIC deployed a new disposal buoy, "PDA 96", to mark the basin feature selected to receive the Royal River material. From October 1 through October 17 , a total of $19,800 \mathrm{~m}^{3}$ of pseudo-UDM was dredged from the anchorage area as well as from the uppermost channel reaches, and disposed at the PDA buoy as part of pseudo-UDM Phase 1 (Figure 2-9). Over the next few days, disposal operations were directed to the DG buoy, northwest of the project area for material dredged from the outer reach. Phase 2 of pseudo-UDM disposal began on October 28 and continued through November 14, with the removal and disposal of sediments from the margins of the anchorage and channel reach 18 , located near the boundary between the upper and middle regions, for a total of $39,500 \mathrm{~m}^{3}$ total pseudo-UDM (Figure 2-9).

\subsubsection{Royal River Project Area Precap (Pseudo-UDM) Survey}

After the second phase of pseudo-UDM deposition, disposal operations were directed to the DG buoy, and a detailed survey of the small pseudo-UDM mound was conducted prior to capping (Figure 2-6). The precap survey consisted of an $800 \mathrm{~m} \times 800 \mathrm{~m}$ bathymetric survey, REMOTS ${ }^{\circledR}$ sediment-profile photography, and sediment grab sampling at the disposal site in November 1996. SAIC compared bathymetric and REMOTS ${ }^{\circledR}$ data obtained during the pseudo-UDM survey operations with the February 1996 baseline datasets to determine mound height, size, and shape, as well as distribution of dredged material within the project area. 


\subsubsection{Capping Dredging Operations, Fall-Winter 1996-97}

After the precap survey in mid-November, capping operations began. Because the outer reaches originally designated to serve as CDM were previously dredged and deposited at the DG buoy, the project design had to be modified. The dredge was moved from the anchorage area and positioned near the mouth of the Royal River to obtain capping material distinct from the pseudo-UDM. Dredging operations proceeded up the river, with barges loading sediments from the middle section and transporting them to the PDS. The sandy sediments near the outer reaches of the river were placed over the pseudo-UDM deposit as the first layers of cap. In cross-section, the horizon of higher sand content material serves as an indicator of the CDM/pseudo-UDM boundary. Later layers of capped material originated from the middle section of the river to the border of the upper reaches that were dredged for pseudo-UDM. From November 21 to December 23,1996 , the clamshell bucket dredge supplied an estimated barge volume of $22,200 \mathrm{~m}^{3}$ of CDM dredged from the middle and outer channel reaches (Figure 2-9).

\subsubsection{Royal River Project Area Postcap (CDM) Survey}

Following the final completion of CDM material placement, SAIC conducted a postcap survey in mid-January 1997 consisting of precision bathymetry and REMOTS ${ }^{\circledR}$ sediment-profile photography to verify accurate placement of the cap material (Figure 2-6). In addition, a series of nine gravity cores were collected over the Royal River mound in early February to obtain cross sections of the capped mound and examine the boundary between the pseudo-UDM and CDM layers.

\subsubsection{Additional Analysis of Royal River Sediment Cores}

The area between the upper and middle reaches of the Royal River, where the areas dredged for pseudo-UDM and CDM intersect (Figure 2-9), was identified as an important area of interest as the cores from the study area were analyzed. The area between Cores RR-15 and RR-26 (Figure 2-4) was not studied in detail during the preliminary Royal River core analysis. To clarify the lithological and biological characteristics of this key transitional area, three more cores from the Royal River were processed and analyzed in the summer of 1997. Cores RR-6, RR-5, and RR-3 were selected from the Royal River archived cores and processed, providing additional evidence for interpretation of cores collected both from the Royal River and the project area (Figure 2-9).

\subsection{Capping Model Predictions}

Based on the amount of dredged material disposed at the PDA buoy during the Portland Capping Experiment $\left(39,500 \mathrm{~m}^{3}\right.$ pseudo-UDM and 22,200 $\left.\mathrm{m}^{3} \mathrm{CDM}\right)$, the 
DAMOS Capping Model predicted the formation of a conical pseudo-UDM deposit approximately $1.2 \mathrm{~m}$ high with flanks extending up to $250 \mathrm{~m}$ from the central point of disposal on a flat bottom. The CDM to pseudo-UDM ratio of $0.56: 1.0$ was low relative to standard capping operations. Typically, a sediment cap of $>0.5 \mathrm{~m}$ in thickness is required over the UDM deposits to provide a sufficient buffer against possible storm-related resuspension and the burrowing of benthic organisms. For this demonstration project, the thickness of capping material covering the pseudo-UDM mound was forecasted to be 20 $\mathrm{cm}$ deep.

The accuracy of mapping the pseudo-UDM and CDM layers on the complex topography of the floor of PDS was expected to be limited, because comparisons between sequential bathymetric surveys are reliable only for detecting changes greater than $20 \mathrm{~cm}$. Single-beam bathymetric surveys conducted over the majority of DAMOS disposal sites yield reliable datasets that provide strong depth difference comparisons. However, these sites are located in areas of flat or gently sloping seafloor. The data collected on the irregular bottom topography of the disposal sites of Maine (CADS, RDS, and PDS) tend to reduce the effectiveness of the standard bathymetric data collection and processing techniques. The complex topography of the Maine sites, a product of glacial scour, yields depth difference plots with a significant number of survey artifacts (Sugden and John 1990). Survey artifacts are false indications of changes in depth due to differences in average grid values resulting from variation in survey vessel track between surveys (Figure 2-10). Because the targeted disposal location had a flat ambient depth relative to the region, survey artifacts were expected to be more limited immediately around the disposal buoy, increasing the success of using single-beam bathymetry at the site.. 


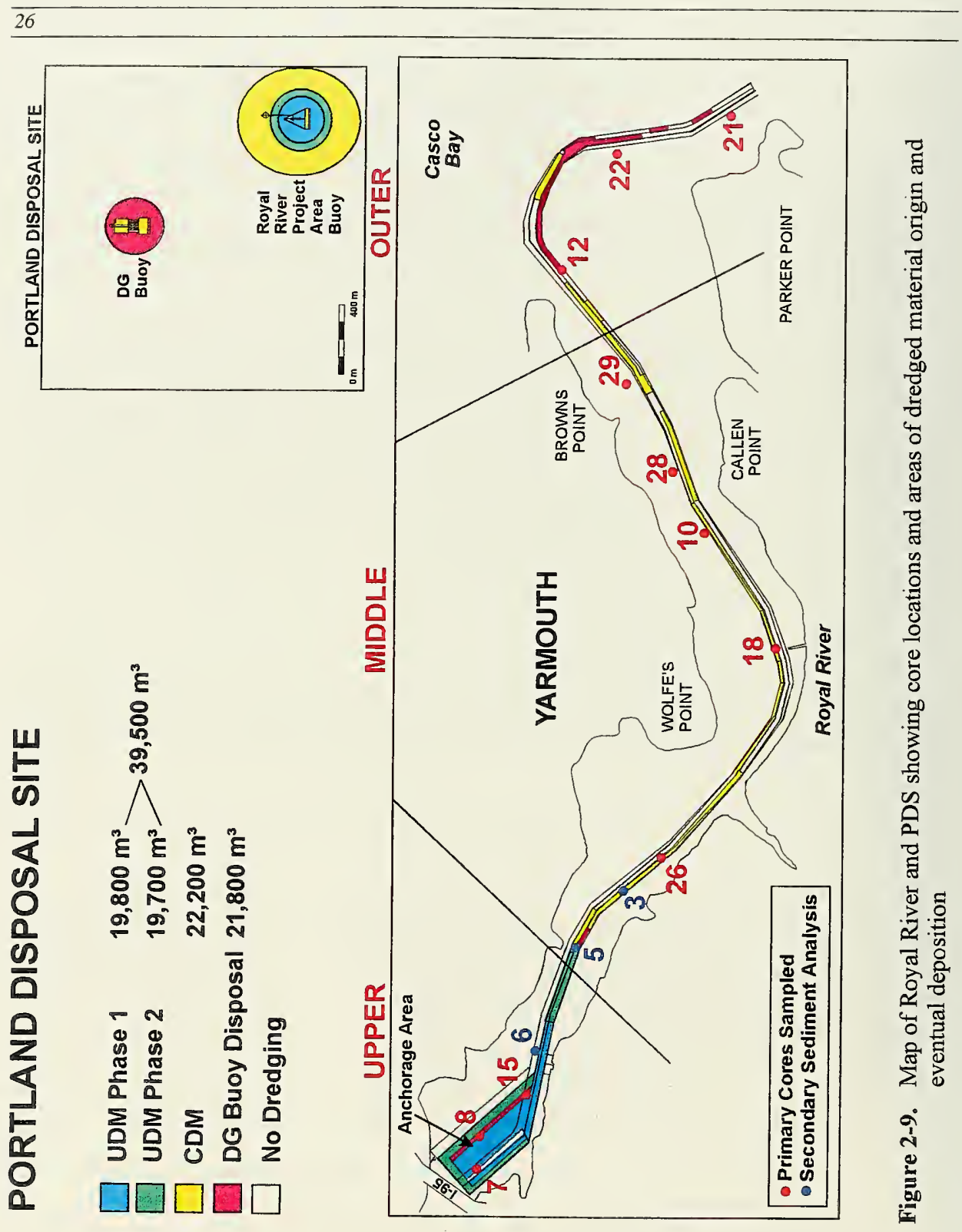




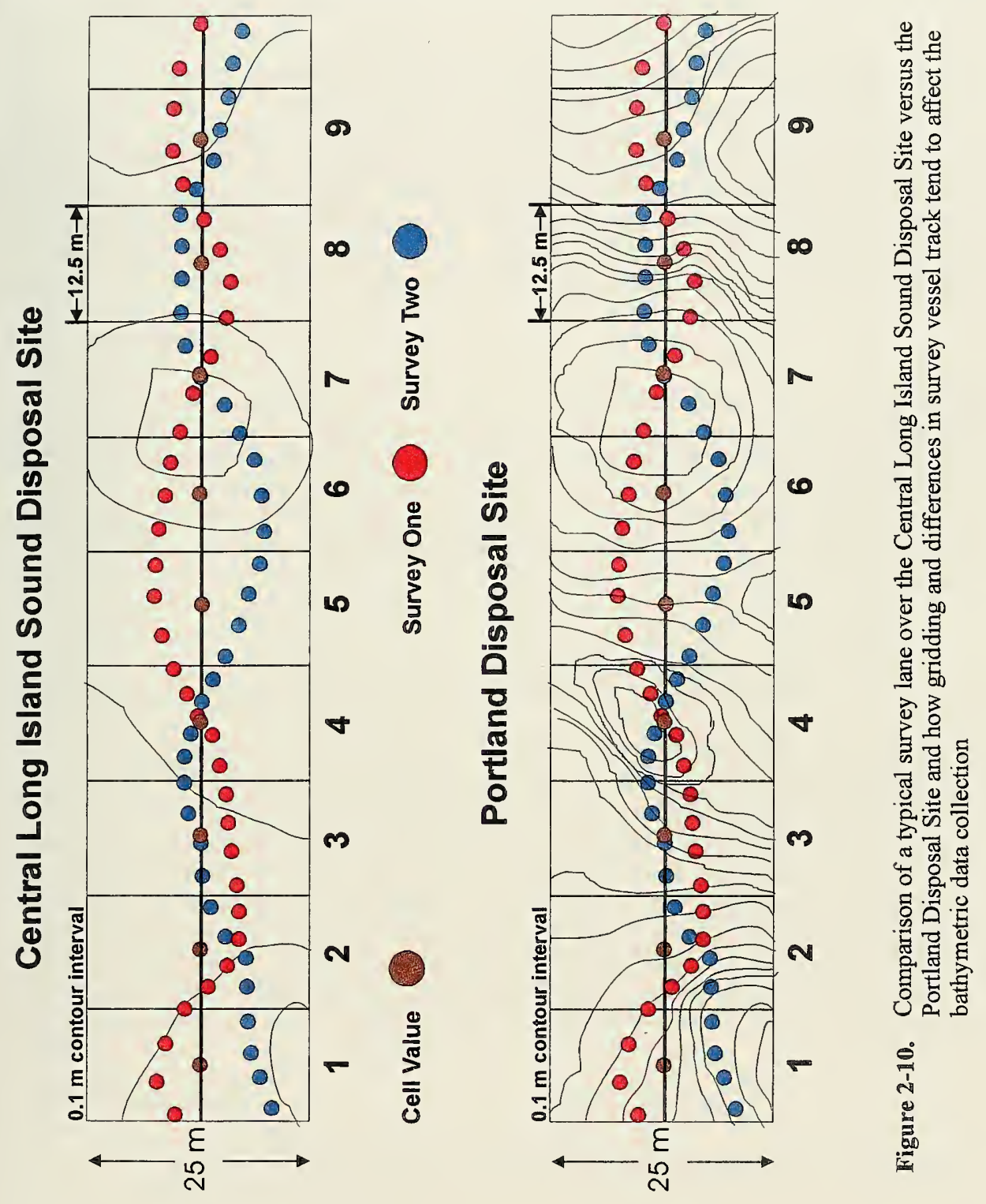


This page left blank intentionally 


\subsection{METHODS}

The Portland Disposal Site Capping Demonstration Project required a wide variety of remote sensors and environmental monitoring techniques to evaluate the effectiveness of subaqueous capping procedures at this deep water disposal site. SAIC conducted five separate field efforts for the project, with one coring survey at the Royal River, and four surveys over the southeast quadrant of PDS as part of the 1996-97 capping operation. Precision bathymetry, REMOTS ${ }^{\circledR}$ sediment-profile and planview photography, side-scan sonar, surface sediment grab sampling, and sediment coring provided information on the ambient conditions at PDS, as well as morphology and composition of the disposal mound.

\subsection{Navigation}

In an effort to provide precise comparisons between the baseline, pseudo-UDM, and CDM survey datasets, all bathymetric data were collected with the use of SAIC's Portable Integrated Navigation and Survey System (PINSS). The PINSS navigation software was resident on a Toshiba ${ }^{\circledR} 3200 X \mathrm{X}$ personal computer (PC) capable of providing real-time navigation, as well as collect position, depth, and time data for later analysis. A Del Norte Trisponder ${ }^{\circledR}$ System provided positioning data referenced to the North American Datum of 1927 (NAD 27) to an accuracy of $\pm 3 \mathrm{~m}$. Shore stations were established along the Maine coast at the known benchmarks of Portland Head Light $\left(43^{\circ} 37.381^{\prime} \mathrm{N}, 70^{\circ} 12.502^{\circ} \mathrm{W}\right)$ and Cape Elizabeth Light ( $43^{\circ} 33.959^{\circ} \mathrm{N}, 70^{\circ} 12.034^{\prime} \mathrm{W}$; Figure 1-5). A detailed description of the navigation system and its operation can be found in the DAMOS Navigation and Bathymetry Reference Report (Murray and Selvitelli 1996).

In order to maximize the efficiency of survey operations, DGPS data in conjunction with PINSS were used to position the survey vessel over the REMOTS ${ }^{\circledR}$ and sediment coring stations. A Magnavox 4200D GPS receiver and a Magnavox MX50R differential beacon receiver provided DGPS positioning data to PINSS referenced to the North American Datum of 1983 (NAD 83) with an accuracy of $\pm 3 \mathrm{~m}$. The Coast Guard differential beacon broadcasting from Brunswick, ME $(316 \mathrm{kHz})$ was used for satellite corrections due to its geographic position relative to PDS. The actual positions of the REMOTS $^{\circledR}$ and sediment stations were later converted to NAD 27 with the U.S. Army Topographic Engineering Center's CORPSCON (version 3.01) for compatibility with bathymetric data. Positions for Royal River sediment cores were collected using DGPS in NAD 83.

\subsection{Survey Areas}

SAIC conducted four bathymetric surveys and three REMOTS ${ }^{\circledR}$ sediment-profile surveys over the $800 \mathrm{~m} \times 800 \mathrm{~m}$ Royal River Project Area centered at $43^{\circ} 33.805^{\circ} \mathrm{N}$ and 
$70^{\circ} 01.614^{\prime} \mathrm{W}$ in the southeast quadrant of PDS (Figure 3-1). The Royal River Project Area bathymetric survey grid consisted of 33 lanes oriented east-west at $25 \mathrm{~m}$ lane spacing. The project area was first occupied during the August 1995 baseline survey, as well as the November 1996 pseudo-UDM and February 1997 CDM bathymetric surveys. A larger $1950 \mathrm{~m} \times 1000 \mathrm{~m}$ area encompassing the southern half of the disposal site was occupied as part of the second baseline bathymetric and side-scan sonar survey (Figure 3-1). Detailed bathymetric charts of the southern regions of PDS were generated and compared during the various stages of capped mound development.

\subsection{Bathymetry}

Precision bathymetry entails the collection of depth soundings along predetermined survey lanes to map seafloor topography, providing information on bottom slopes as well as geological and sedimentological features. Sequential bathymetric surveys that occupy the same area of seafloor are valuable in detecting and quantifying changes in bottom topography over time. By calculating the changes in depth between two individual bathymetric surveys (depth differencing), accumulation of disposed dredged material or the reduction in mound height due to consolidation or erosion can be measured.

The DAMOS Program generally uses single-beam bathymetry, which provides precise depth data ( $\pm 0.05 \%$ of overall depth) for the seafloor directly under the survey vessel (Murray and Selvitelli 1996). For the PDS surveys, the individual soundings were averaged and gridded within $12.5 \mathrm{~m} \times 25 \mathrm{~m}$ cells, yielding a digital depth matrix. Contour charts and three-dimensional representations of the bottom were then produced through interpolation between gridded values. Depth difference plots are generated by comparing corresponding gridded values to detect changes on the seafloor.

Efforts to minimize the development of survey artifacts formed by differences in survey vessel track or configuration within the sequential bathymetric surveys were made. One research vessel, with identical survey configurations, was used to complete the data collection efforts during the February 1996 baseline survey, as well as the precap and postcap surveys. In addition, all four bathymetric surveys were performed with the same navigation, data collection, and data processing software.

\subsubsection{Bathymetric Data Collection}

An ODOM DF3200 Echotrac ${ }^{\circledR}$ Survey Fathometer with a narrow beam, $208 \mathrm{kHz}$ transducer measured individual depths to a resolution of $3.0 \mathrm{~cm}(0.1 \mathrm{ft})$ as described in the DAMOS Navigation and Bathymetry Reference Report (Murray and Selvitelli 1996). Depth values transmitted to PINSS were adjusted for transducer depth. The acoustic returns of the fathometer can reliably detect changes in depth of $20 \mathrm{~cm}$ or greater due to 


\section{Portland Disposal Site Capping Demonstration Project Bathymetric Survey Areas}

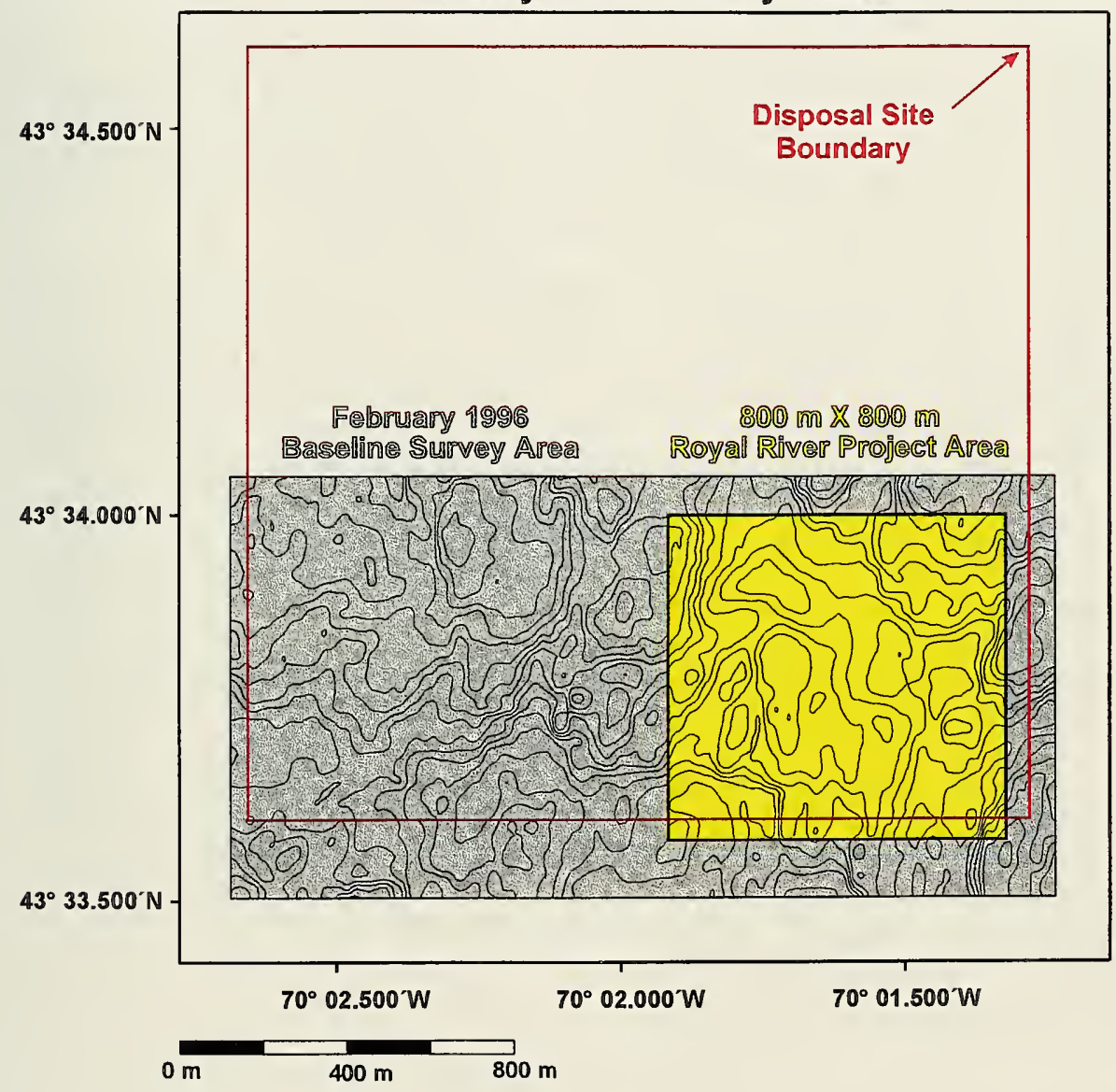

Figure 3-1. Base map showing the $1950 \mathrm{~m} \times 1000 \mathrm{~m}$ bathymetric survey area during the February 1996 baseline survey (NAD 27) in relation to the Royal River Project Area (yellow) at PDS, $2.0 \mathrm{~m}$ contour interval (MLLW) 
the accumulation of errors introduced by the positioning system, vertical motion of the survey vessel, changes in sound velocity through the water column, the slope of the bottom, and tidal corrections.

Observed tidal data were obtained through the National Oceanographic and Atmospheric Administration (NOAA), Ocean and Lake Levels Division's (OLLD) National Water Level Observation Network. This network is composed of 181 water level stations that are located throughout the Great Lakes and coastal regions of United States interest. These stations are equipped with the Next Generation Water Level Measurement System tide gauges and satellite transmitters that have collected and transmitted tide data to the central NOAA facility every six minutes, since 1 January 1994.

Observed tide data are available 1 to 6 hours from the time of collection in a station datum or referenced to mean lower low water (MLLW) and based on Coordinated Universal Time (UTC). For the 1995 and 1996 PDS surveys, data from NOAA tide station 8418150 in Casco Bay, Portland, ME, were used for tidal calculations. The NOAA 6-minute tide data were downloaded in the MLLW datum, corrected to local time, and tidal differences based on Potts Harbor, South Harpswell Neck, were applied.

During the bathymetric survey, a Seabird Instruments, Inc. SBE 26-03 Sea Gauge wave and tide recorder was used to collect tidal data on site. The tide gauge, deployed in the survey area, recorded pressure values every six minutes. After conversion, the pressure readings provided a constant record of tidal variations in the survey area. These observed tidal data were later used to compare and verify the corrected NOAA data generated from the Portland station (Figure 3-2).

A Seabird Instruments, Inc. SEACAT SBE 19-01 Conductivity, Temperature, and Depth (CTD) probe was used to obtain sound velocity measurements at the start, midpoint, and end of each survey day. The data collected by the CTD probe were bin-averaged to 1 meter depth intervals to account for any pycnoclines, rapid changes in density that create distinct layers within the water column. Sound velocity correction factors were then calculated using the bin-averaged values.

\subsubsection{Bathymetric Data Processing}

The bathymetric data were analyzed using SAIC's Hydrographic Data Analysis System (HDAS), version 1.03. Raw bathymetric data were imported into HDAS, corrected for sound velocity, and standardized to MLLW using the NOAA observed tides. The bathymetric data were then used to construct depth models of the surveyed area. A detailed discussion of the bathymetric analysis technique is provided in the DAMOS Bathymetry and Navigation Reference Report (Murray and Selvitelli 1996). 


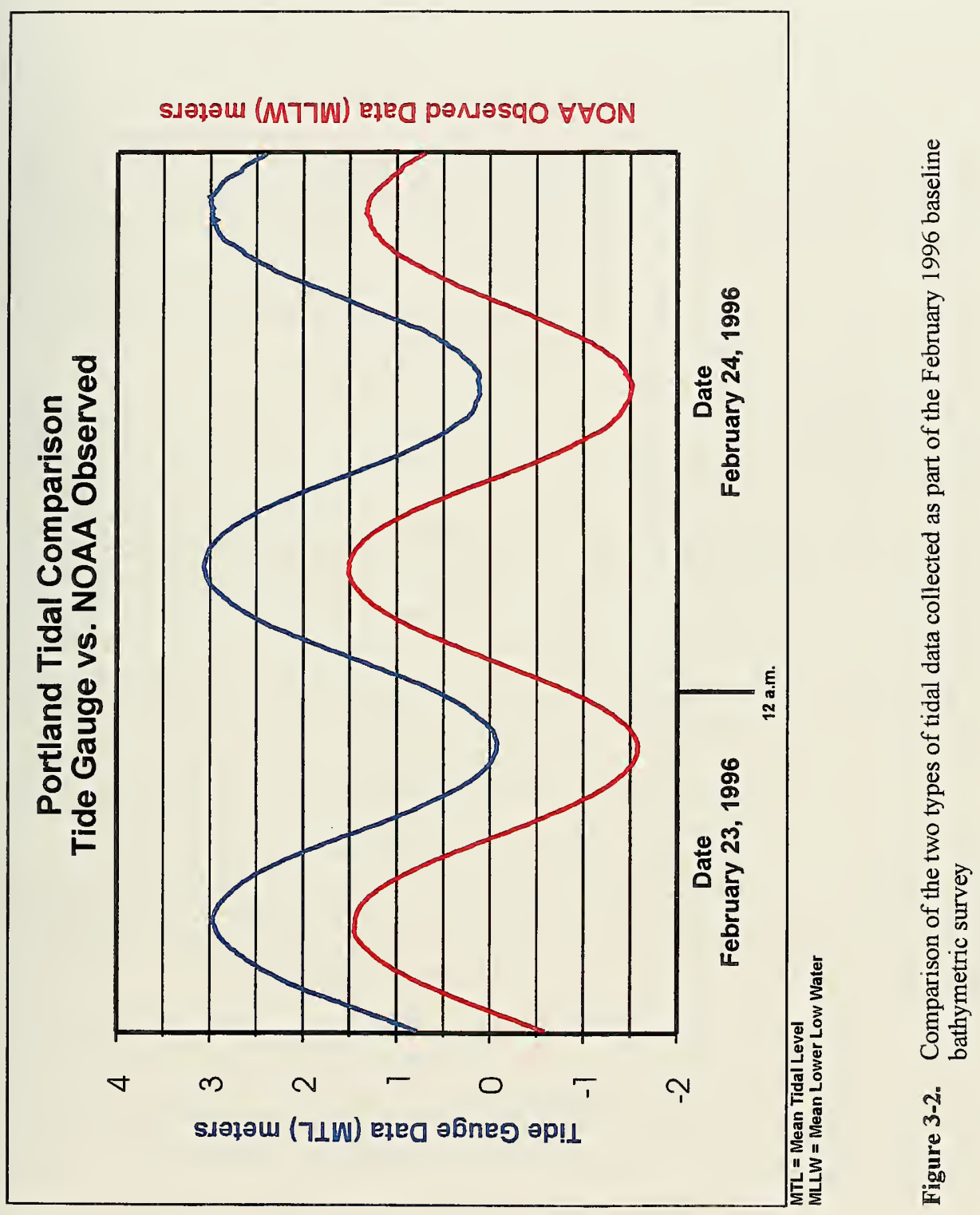




\subsection{Side-Scan Sonar}

Side-scan sonar data were used to remotely characterize the entire southern region of the PDS seafloor during the February 1996 baseline survey. The high resolution sidescan sonar survey was performed concurrently with the bathymetric data collection efforts. The side-scan sonar survey lanes were spaced at $100 \mathrm{~m}$ intervals, and the towfish altitude was controlled to insure 150 percent bottom coverage over the expanded February 1996 survey area. Acoustic signals at a frequency of $100 \mathrm{kHz}$ were emitted from two transducers mounted in a Klein $422 \mathrm{~S}$ dual-frequency $(100 \mathrm{kHz}$ and $500 \mathrm{kHz})$ towfish. The acoustic returns were relayed to a Klein 595 side-scan sonar data recorder and thermal printer to produce images of the bottom features at PDS.

\subsection{Photography}

\subsubsection{REMOTS ${ }^{\circledast}$ Sediment-Profile Photography}

For the Portland Disposal Site Capping Demonstration Project, the REMOTS ${ }^{\circledR}$ sediment-profile photography supplemented the bathymetric data, minimizing the limitations of single-beam bathymetry over PDS. The REMOTS ${ }^{\circledR}$ camera provided undisturbed profile images of the top $20 \mathrm{~cm}$ of sediment to obtain information on the physical and biological composition at the sediment-water interface (Figure 3-3). For the capping project, REMOTS ${ }^{\circledR}$ photography was primarily used to map the distribution of dredged material layers over the Royal River Project Area, measuring thin sediment strata as part of the baseline, pseudo-UDM, and CDM stages of mound development.

A Benthos ${ }^{\circledR}$ Model 3731 sediment-profile camera was used to sample the surficial sediment layers and track the distribution of dredged material within the Royal River Project Area. Cross-sectional photographs were collected for detailed analysis and intercomparison of a variety of physical characteristics. A series of 33 REMOTS $^{\circledR}$ camera stations were established over the project area and occupied during three of the four PDS surveys (February 1996 baseline, pseudo-UDM, and CDM). Three replicate photographs were taken within a $20 \mathrm{~m}$ radius of each target REMOTS ${ }^{\circledR}$ camera station.

The REMOTS ${ }^{\circledR}$ sampling grid over the Royal River Project Area was designed to form a modified star-shaped pattern, radiating from the PDA 95 and PDA 96 disposal buoy positions (Figure 3-4). The sampling scheme was established with respect to the bathymetric features of the $800 \mathrm{~m} \times 800 \mathrm{~m}$ project area and remained constant throughout the demonstration project distributed within the likely pattern of dredged material accumulation (Table 3-1). 
REMOTS $^{\circledR}$ data collection activities were abbreviated by the onset of a winter storm during the February 1996 baseline survey. Three replicate photographs were obtained from 24 stations surrounding the PDA buoy, with deteriorating weather conditions precluding the collection of images from the final nine stations. All 33 stations were occupied during the subsequent November 1996 precap and postcap surveys. All REMOTS $^{\circledR}$ sediment-profile photographs were analyzed for the presence of dredged material and its sedimentary characteristics. A time series, based on evidence of dredged material deposition, was then constructed for each station from the REMOTS ${ }^{\circledR}$ photographs.

\subsubsection{Planview Photography}

As part of the February 1996 baseline REMOTS ${ }^{\circledast}$ survey over the Royal River Project Area, a series of planview photographs of the PDS seafloor were obtained. The sediment-profile camera frame was equipped with a Photosea ${ }^{\circledR} 1000 \mathrm{~A}$ Underwater Camera System and deployed along the NW/SE transect of the REMOTS ${ }^{\circledR}$ sampling grid. An independent trigger mechanism allowed the planview camera and strobe to fire 0.5 to 1.0 seconds before the REMOTS ${ }^{\circledR}$ camera touched down, providing undisturbed images of the bottom. The planview photographs were later correlated with the corresponding REMOTS ${ }^{\circledR}$ sediment-profile data through time and replicate notations for point characterization of the PDS seafloor (Figure 3-5). 
Table 3-1

REMOTS $^{\circledR}$ Sampling Grid

\begin{tabular}{|c|c|c|}
\hline \multicolumn{3}{|c|}{ REMOTS $®$ Sampling Grid } \\
\hline Station & Latitude & Longitude \\
\hline \multicolumn{3}{|c|}{ NAD 27} \\
\hline CTR & $43^{\circ} 33.790^{\prime} \mathrm{N}$ & $70^{\circ} 01.512^{\prime} \mathrm{W}$ \\
\hline $50 \mathrm{~N}$ & $43^{\circ} 33.817^{\prime} \mathrm{N}$ & $70^{\circ} 01.512^{\prime} \mathrm{W}$ \\
\hline $100 \mathrm{~N}$ & $43^{\circ} 33.844^{\prime} \mathrm{N}$ & $70^{\circ} 01.512^{\prime} \mathrm{W}$ \\
\hline $200 \mathrm{~N}$ & $43^{\circ} 33.898^{\prime} \mathrm{N}$ & $70^{\circ} 01.512^{\prime} \mathrm{W}$ \\
\hline 50NE & $43^{\circ} 33.809^{\prime} \mathrm{N}$ & $70^{\circ} 01.488^{\circ} \mathrm{W}$ \\
\hline $100 \mathrm{NE}$ & $43^{\circ} 33.828^{\prime} \mathrm{N}$ & $70^{\circ} 01.464^{\prime} \mathrm{W}$ \\
\hline $200 \mathrm{NE}$ & $43^{\circ} 33.866^{\circ} \mathrm{N}$ & $70^{\circ} 01.410^{\circ} \mathrm{W}$ \\
\hline 50ENE & $43^{\circ} 33.800^{\circ} \mathrm{N}$ & $70^{\circ} 01.482^{\prime} \mathrm{W}$ \\
\hline $100 \mathrm{ENE}$ & $43^{\circ} 33.810^{\circ} \mathrm{N}$ & $70^{\circ} 01.446^{\prime} \mathrm{W}$ \\
\hline 200ENE & $43^{\circ} 33.831^{\prime} \mathrm{N}$ & $70^{\circ} 01.380^{\circ} \mathrm{W}$ \\
\hline 75ESE & $43^{\circ} 33.783^{\circ} \mathrm{N}$ & $70^{\circ} 01.458^{\circ} \mathrm{W}$ \\
\hline 125ESE & $43^{\circ} 33.778^{\circ} \mathrm{N}$ & $70^{\circ} 01.422^{\circ} \mathrm{W}$ \\
\hline 50SE & $43^{\circ} 33.771^{\prime} \mathrm{N}$ & $70^{\circ} 01.488^{\circ} \mathrm{W}$ \\
\hline 100SE & $43^{\circ} 33.752^{\prime} \mathrm{N}$ & $70^{\circ} 01.464^{\prime} \mathrm{W}$ \\
\hline 200SE & $43^{\circ} 33.713^{\prime} \mathrm{N}$ & $70^{\circ} 01.410^{\circ} \mathrm{W}$ \\
\hline 300SE & $43^{\circ} 33.676^{\circ} \mathrm{N}$ & $70^{\circ} 01.356^{\prime} \mathrm{W}$ \\
\hline $400 \mathrm{SE}$ & $43^{\circ} 33.637^{\circ} \mathrm{N}$ & $70^{\circ} 01.302^{\circ} \mathrm{W}$ \\
\hline $50 \mathrm{~S}$ & $43^{\circ} 33.763^{\prime} \mathrm{N}$ & $70^{\circ} 01.512^{\prime} \mathrm{W}$ \\
\hline $100 \mathrm{~S}$ & $43^{\circ} 33.736^{\prime} \mathrm{N}$ & $70^{\circ} 01.512^{\prime} \mathrm{W}$ \\
\hline $200 \mathrm{~S}$ & $43^{\circ} 33.682^{\prime} \mathrm{N}$ & $70^{\circ} 01.512^{\prime} \mathrm{W}$ \\
\hline $300 \mathrm{~S}$ & $43^{\circ} 33.628^{\prime} \mathrm{N}$ & $70^{\circ} 01.512^{\prime} \mathrm{W}$ \\
\hline $400 \mathrm{~S}$ & $43^{\circ} 33.574^{\prime} \mathrm{N}$ & $70^{\circ} 01.512^{\prime} \mathrm{W}$ \\
\hline $50 \mathrm{SW}$ & $43^{\circ} 33.771^{\circ} \mathrm{N}$ & $70^{\circ} 01.542^{\prime} \mathrm{W}$ \\
\hline $100 \mathrm{SW}$ & $43^{\circ} 33.752^{\prime} \mathrm{N}$ & $70^{\circ} 01.566^{\prime} \mathrm{W}$ \\
\hline $200 \mathrm{SW}$ & $43^{\circ} 33.713^{\circ} \mathrm{N}$ & $70^{\circ} 01.620^{\circ} \mathrm{W}$ \\
\hline $50 \mathrm{~W}$ & $43^{\circ} 33.790^{\circ} \mathrm{N}$ & $70^{\circ} 01.554^{\prime} \mathrm{W}$ \\
\hline $100 \mathrm{~W}$ & $43^{\circ} 33.790^{\circ} \mathrm{N}$ & $70^{\circ} 01.590^{\circ} \mathrm{W}$ \\
\hline $200 \mathrm{~W}$ & $43^{\circ} 33.790^{\circ} \mathrm{N}$ & $70^{\circ} 01.662^{\circ} \mathrm{W}$ \\
\hline $50 \mathrm{NW}$ & $43^{\circ} 33.808^{\prime} \mathrm{N}$ & $70^{\circ} 01.542^{\prime} \mathrm{W}$ \\
\hline $100 \mathrm{NW}$ & $43^{\circ} 33.828^{\circ} \mathrm{N}$ & $70^{\circ} 01.566^{\prime} \mathrm{W}$ \\
\hline $200 \mathrm{NW}$ & $43^{\circ} 33.866^{\circ} \mathrm{N}$ & $70^{\circ} 01.620^{\circ} \mathrm{W}$ \\
\hline $300 \mathrm{NW}$ & $43^{\circ} 33.904^{\prime} \mathrm{N}$ & $70^{\circ} 01.674^{\circ} \mathrm{W}$ \\
\hline $400 \mathrm{NW}$ & $43^{\circ} 33.943^{\prime} \mathrm{N}$ & $70^{\circ} 01.722^{\prime} \mathrm{W}$ \\
\hline
\end{tabular}




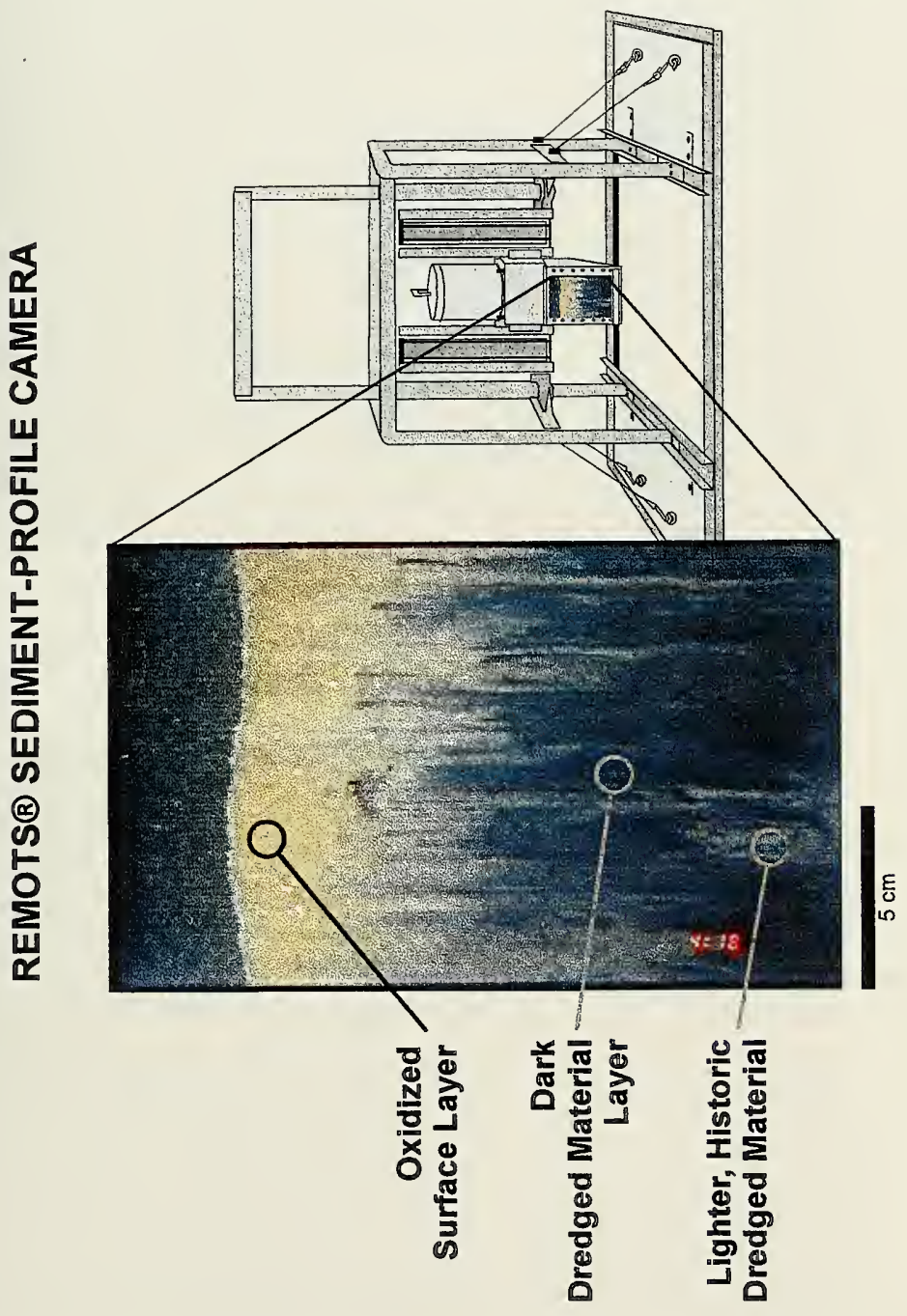

告 


\section{Portland Disposal Site Capping Demonstration Project REMOTS $®$ Sediment-Profile Photography Stations}

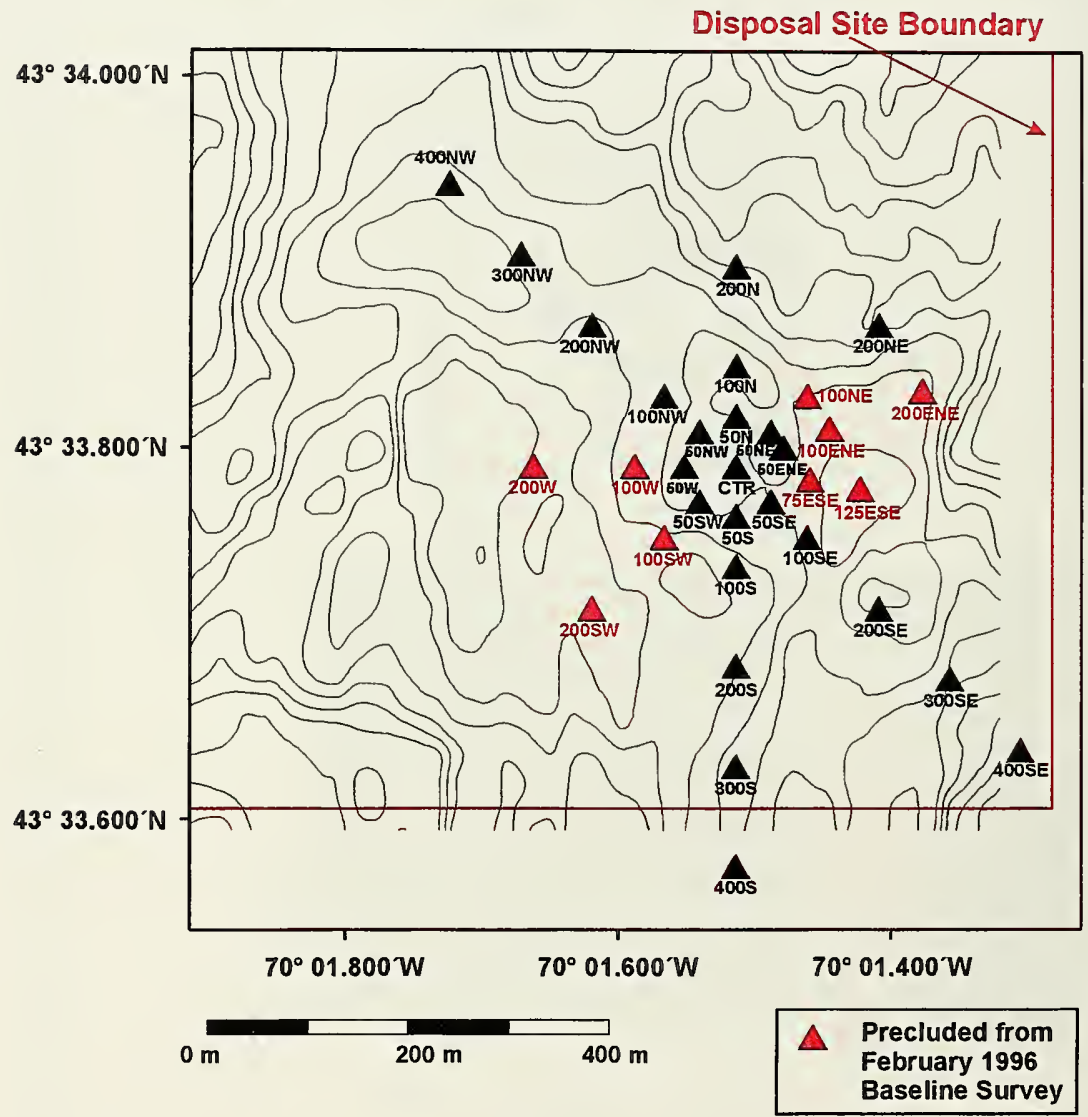

Figure 3-4. Bathymetric chart of the Royal River Project Area (NAD 27) showing REMOTS $^{\circledR}$ sediment-profile station locations relative to the disposal site boundary 

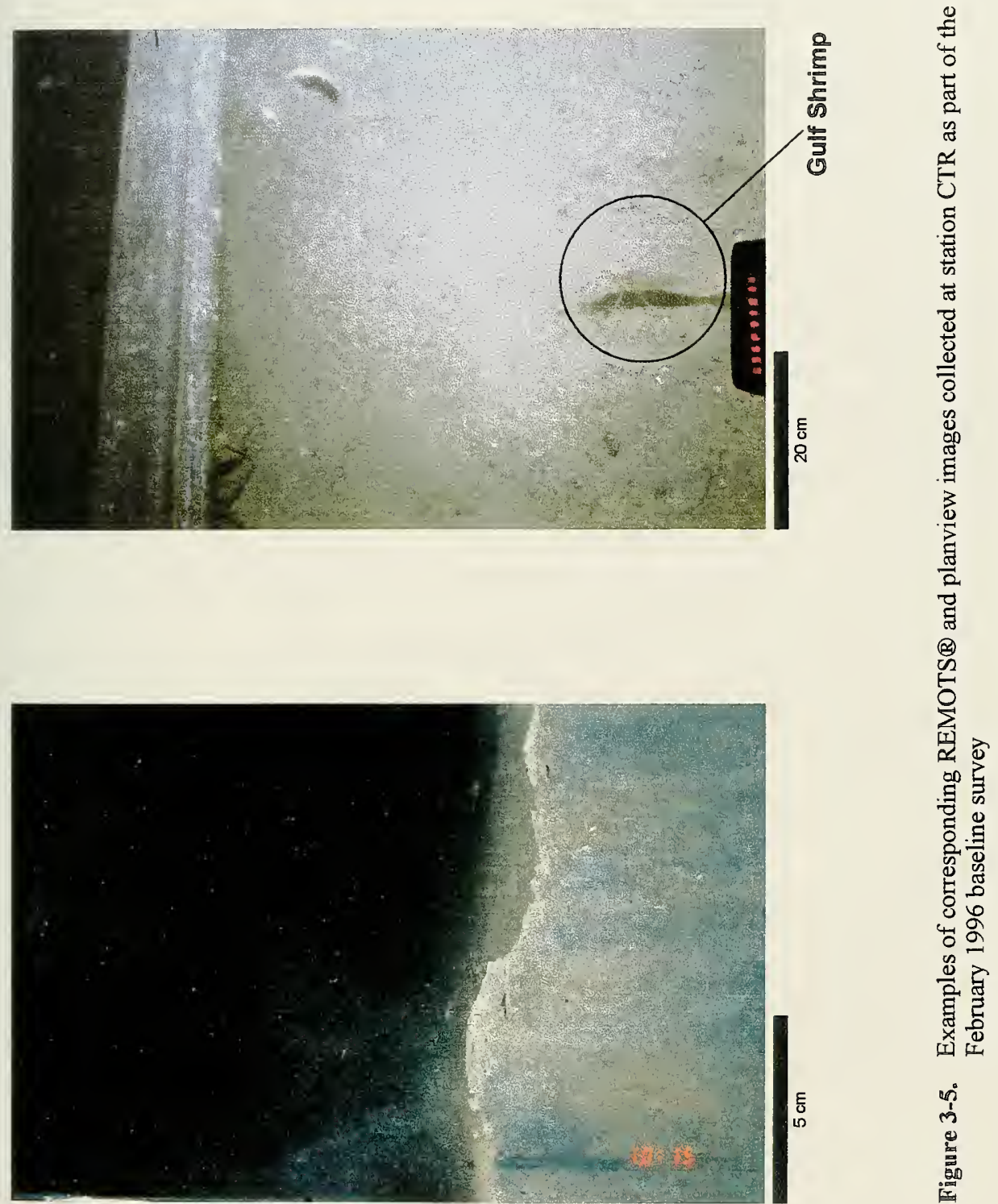


\subsection{Sediment Grab Sample Collection}

A series of nine sediment grab samples were collected from the initial pseudo-UDM deposit during the November 1996 precap survey over the Royal River Project Area (Figure 3-6). A $0.1 \mathrm{~m}^{2}$ Young-modified Van Veen grab sampling device was deployed at the central disposal point as well as eight stations within a $50 \mathrm{~m}$ radius of the PDA 96 buoy (Table 3-2).

Each grab sample from the precap survey provided sufficient quantity of material for analyses. For grain size analyses, a portion of the sediments were placed into onegallon Dow ${ }^{\circledR}$ Ziploc $^{\circledR}$ storage bags, labeled, and forwarded to an independent laboratory. The remaining sediments of each grab sample were subdivided into two pre-cleaned 1 liter bottles, and preserved in a $70 \%$ methanol/seawater or a buffered Rose Bengal/formalin/seawater solution. A discussion of the solutions used for preserving the samples is in Section 3.8. The 18 samples were sent to the Micropaleontology Laboratory at Wesleyan University for meiofauna and mineralogical analyses.

Table 3-2

\begin{tabular}{|c|c|c|}
\hline \multicolumn{3}{|c|}{ Grab Sampling Grid } \\
\hline Station & Latitude & Longitude \\
\hline \multicolumn{3}{|c|}{ NAD 27} \\
\hline CTR & $43^{\circ} 33.790^{\prime} \mathrm{N}$ & $70^{\circ} 01.512^{\prime} \mathrm{W}$ \\
\hline $50 \mathrm{~N}$ & $43^{\circ} 33.817^{\prime} \mathrm{N}$ & $70^{\circ} 01.512^{\prime} \mathrm{W}$ \\
\hline $50 \mathrm{NE}$ & $43^{\circ} 33.830^{\prime} \mathrm{N}$ & $70^{\circ} 01.488^{\prime} \mathrm{W}$ \\
\hline 50ENE & $43^{\circ} 33.800^{\prime} \mathrm{N}$ & $70^{\circ} 01.482^{\prime} \mathrm{W}$ \\
\hline 50SE & $43^{\circ} 33.771^{\prime} \mathrm{N}$ & $70^{\circ} 01.488^{\prime} \mathrm{W}$ \\
\hline $50 \mathrm{~S}$ & $43^{\circ} 33.763^{\circ} \mathrm{N}$ & $70^{\circ} 01.512^{\prime} \mathrm{W}$ \\
\hline $50 \mathrm{SW}$ & $43^{\circ} 33.771^{\prime} \mathrm{N}$ & $70^{\circ} 01.542^{\prime} \mathrm{W}$ \\
\hline $50 W$ & $43^{\circ} 33.790^{\prime} \mathrm{N}$ & $70^{\circ} 01.554^{\prime} \mathrm{W}$ \\
\hline $50 N W$ & $43^{\circ} 33.830^{\prime} \mathrm{N}$ & $70^{\circ} 01.542^{\prime} \mathrm{W}$ \\
\hline
\end{tabular}




\section{Portland Disposal Site Grab Samples and Gravity Core Locations}

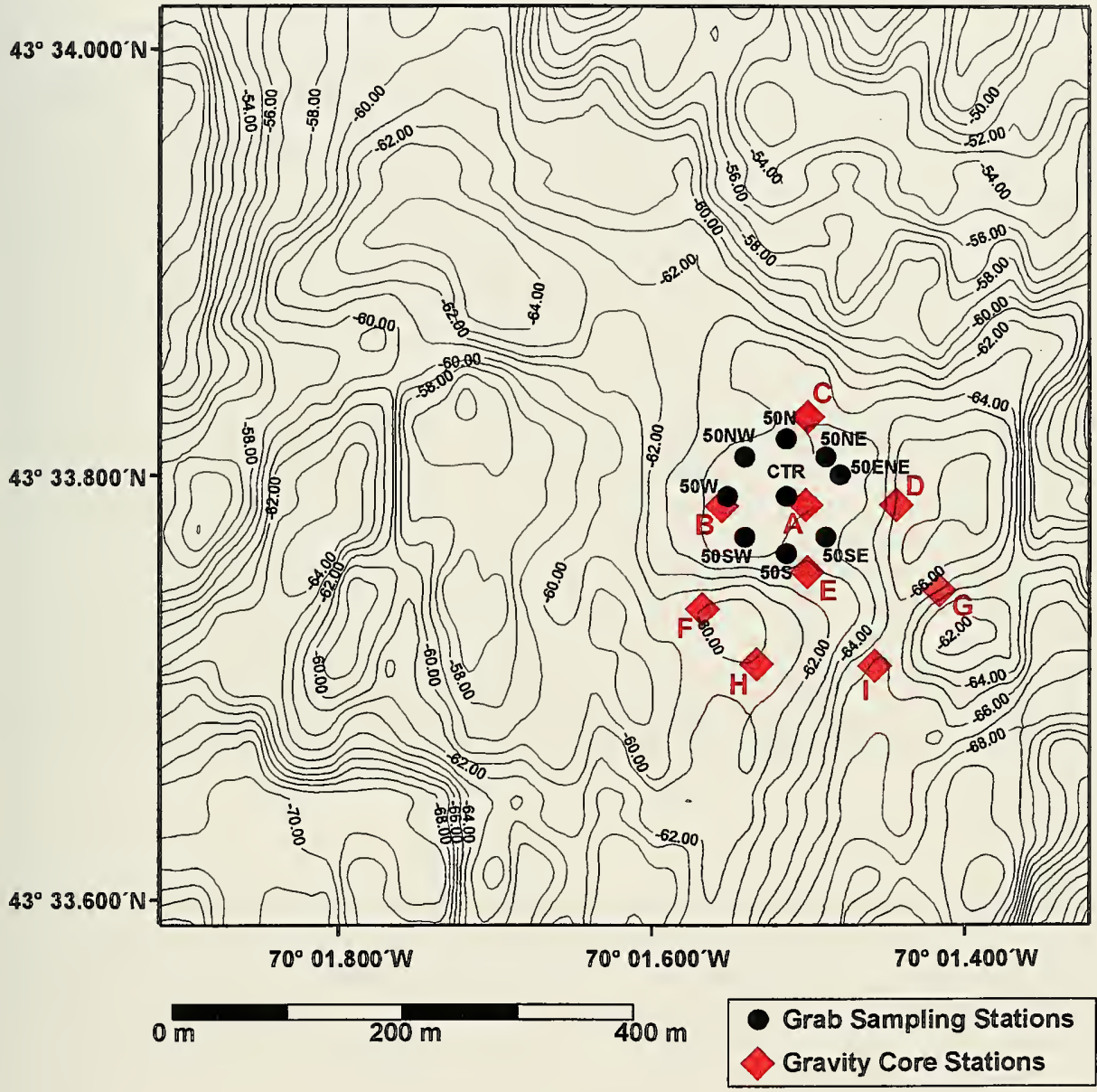

Figure 3-6. Bathymetric chart of the Royal River Project Area (NAD 27) showing locations of grab sampling and gravity core stations 


\subsection{Sediment Coring Collection}

\subsubsection{Royal River Survey}

Thirty vibracores (RR-1 through RR-30) were collected in the Royal River in August 1995 from the base of the falls at the Interstate-95 highway overpass to Parker Point (Figure 2-4). The cores provided deep cross sections of the material to be dredged from the Royal River (Table 3-3). Sediment cores were collected to a depth of approximately $2.5 \mathrm{~m}$ with a custom, concrete compactor-type vibracore unit, deployed from a shallow draft vessel. The sediment core was retained within a rinsed polyethylene core liner, capped, and stored vertically during field operations. Twice a day, cores were transported from the vessel to refrigerated storage containers on shore. Following the survey, the cores were relocated to a refrigerated storage facility at the University of Rhode Island's Graduate School of Oceanography (GSO), and stored for later analysis.

SAIC processed the Royal River vibracores at the GSO coring laboratory in September 1995. Core liners were split lengthwise using a hydraulic core splitter, and nylon wire was used to divide the sediment samples. One-half of each core was photographed, visually described, and archived for potential future analyses. A subset of eleven core halves were selected and sampled for use in the identification of tracer components for the Portland Disposal Site Capping Demonstration Project. Sediment samples were collected from Cores RR-7, RR-8, RR-10, RR-12, RR-15, RR-18, RR-21, RR-22, RR-26, RR-28, and RR-29. The composited sediment samples were placed in a $70 \%$ methanol/seawater solution. After the meiofauna had been preserved, the samples were washed, sieved, and dried. Eleven samples containing the fine fraction, particles 500 $\mu \mathrm{m}$ to $63 \mu \mathrm{m}$ in size, were sent to the Micropaleontology Laboratory at Wesleyan University.

\subsubsection{Postcap Survey}

As part of the January 1997 CDM survey over the Royal River Project Area, nine gravity cores were collected to verify the presence of a pseudo-UDM/CDM interface within the capped mound (Figure 3-6). Nine stations were selected over the project area based on the pattern of pseudo-UDM accumulation (Table 3-4). The target location for Core A was established over the PDA 96 buoy position. The remaining core locations were strategically placed relative to the morphology of the pseudo-UDM deposit. Cores E and $\mathrm{G}$ were situated over the thicker portions of the mound, Cores $\mathrm{D}$ and $\mathrm{F}$ were located at the margins of the detectable mound, and Cores $\mathrm{B}, \mathrm{H}$, and I sampled the apron of the deposit. Core $\mathrm{C}$ was collected away from the accumulation of Royal River pseudo-UDM to characterize the thin strata of dredged material, as well as to ensure penetration into ambient sediments. 
Table 3-3

Royal River Core Data

\begin{tabular}{|c|c|c|c|c|c|c|c|c|c|c|}
\hline \multirow[t]{2}{*}{ Core Name } & \multicolumn{2}{|c|}{ Latitude } & \multicolumn{2}{|c|}{ Longitude } & \multirow[t]{2}{*}{$\begin{array}{c}\text { Total } \\
\text { Length } \\
\text { (cm) } \\
\end{array}$} & \multirow[t]{2}{*}{ Date } & \multirow[t]{2}{*}{ Time } & \multirow[t]{2}{*}{$\begin{array}{c}\text { Water } \\
\text { Depth } \\
\text { (ft) }\end{array}$} & \multirow[t]{2}{*}{ Tide (ft) } & \multirow[t]{2}{*}{$\begin{array}{c}\text { Required } \\
\text { Dredge }(\mathrm{cm})\end{array}$} \\
\hline & degrees & minutes & & & & & & & & \\
\hline RR-1 & 43 & 47.832 & 70 & 10.509 & 184 & $8 / 9 / 95$ & 910 & 11.5 & 9 & 168 \\
\hline RR-2 & 43 & 47.768 & 70 & 10.483 & 185 & $8 / 9 / 95$ & 950 & 15.5 & 9.3 & 55 \\
\hline RR-3 & 43 & 47.603 & 70 & 9.99 & 208 & $8 / 9 / 95$ & 1055 & 9.5 & 9.6 & 247 \\
\hline $\mathrm{RR}-4$ & 43 & 47.627 & 70 & 10.015 & 210 & $8 / 9 / 95$ & 1135 & 9.5 & 9 & 229 \\
\hline RR-5 & 43 & 47.673 & 70 & 10.106 & 177 & $8 / 9 / 95$ & 1200 & 11 & 8 & 152 \\
\hline RR-6 & 43 & 47.733 & 70 & 10.317 & 91 & $8 / 9 / 95$ & 1245 & 13 & 6.8 & 55 \\
\hline RR-7 & 43 & 47.819 & 70 & 10.554 & 117 & $8 / 9 / 95$ & 1550 & 4.5 & 0 & 107 \\
\hline RR-8 & 43 & 47.815 & 70 & 10.49 & 118 & $8 / 9 / 95$ & 1705 & 2 & 0 & 183 \\
\hline RR-9 & 43 & 47.619 & 70 & 8.879 & 156 & $8 / 10 / 95$ & 750 & 11.5 & 3.5 & 0 \\
\hline RR-10 & 43 & 47.484 & 70 & 9.268 & 189 & $8 / 10 / 95$ & 855 & 11.5 & 6.6 & 94 \\
\hline RR-11 & 43 & 47.535 & 70 & 9.063 & 110 & $8 / 10 / 95$ & 930 & 17 & 8 & -30 \\
\hline RR-12 & 43 & 47.701 & 70 & 8.74 & 195 & $8 / 10 / 95$ & 1025 & 17 & 9.2 & 6 \\
\hline RR-13 & 43 & 47.735 & 70 & 8.535 & 221 & $8 / 10 / 95$ & 1110 & 12 & 10 & 183 \\
\hline RR-14 & 43 & 47.75 & 70 & 10.352 & 177 & $8 / 10 / 95$ & 1310 & 9 & 8 & 213 \\
\hline RR-15 & 43 & 47.746 & 70 & 10.406 & 98 & $8 / 10 / 95$ & 1340 & 13 & 7 & 61 \\
\hline RR-16 & 43 & 47.587 & 70 & 9.956 & 199 & $8 / 10 / 95$ & 1425 & 13 & 4.5 & -15 \\
\hline RR-17 & 43 & 47.461 & 70 & 9.778 & 183 & $8 / 10 / 95$ & 1445 & 12.5 & 4 & -15 \\
\hline RR-18 & 43 & 47.378 & 70 & 9.499 & 114 & $8 / 10 / 95$ & 1510 & 5 & 2.8 & 177 \\
\hline RR-19 & 43 & 47.424 & 70 & 9.361 & 153 & $8 / 10 / 95$ & 1535 & 8 & 2 & 61 \\
\hline RR-20 & 43 & 47.645 & 70 & 8.485 & 89 & $8 / 10 / 95$ & 1610 & 9 & 0.4 & -18 \\
\hline RR-21 & 43 & 47.449 & 70 & 8.427 & 176 & $8 / 10 / 95$ & 1650 & 6 & -0.4 & 49 \\
\hline RR-22 & 43 & 47.62 & 70 & 8.505 & 194 & $8 / 11 / 95$ & 815 & 6 & 2 & 122 \\
\hline RR-23 & 43 & 47.705 & 70 & 8.531 & 192 & $8 / 11 / 95$ & 840 & 9 & 4 & 91 \\
\hline RR-24 & 43 & 47.627 & 70 & 8.901 & 178 & $8 / 11 / 95$ & 905 & 11 & 4.5 & 46 \\
\hline RR-25 & 43 & 47.394 & 70 & 9.666 & 163 & $8 / 11 / 95$ & 940 & 10 & 6 & 122 \\
\hline RR-26 & 43 & 47.546 & 70 & 9.924 & 173 & $8 / 11 / 95$ & 1005 & 8 & 7 & 213 \\
\hline RR-27 & 43 & 47.511 & 70 & 9.11 & 66 & $8 / 11 / 95$ & 1030 & 10.5 & 8 & 168 \\
\hline RR-28 & 43 & 47.533 & 70 & 9.144 & 176 & $8 / 11 / 95$ & 1100 & 11 & 9 & 183 \\
\hline RR-29 & 43 & 47.603 & 70 & 8.967 & 194 & $8 / 11 / 95$ & 1225 & 10 & 10.4 & 256 \\
\hline RR-30 & 43 & 47.622 & 70 & 10.015 & 197 & $8 / 11 / 95$ & 1340 & 9.5 & 7.5 & 183 \\
\hline
\end{tabular}

All Coordinates provided in NAD 83. 
Table 3-4

PDS Gravity Core Locations

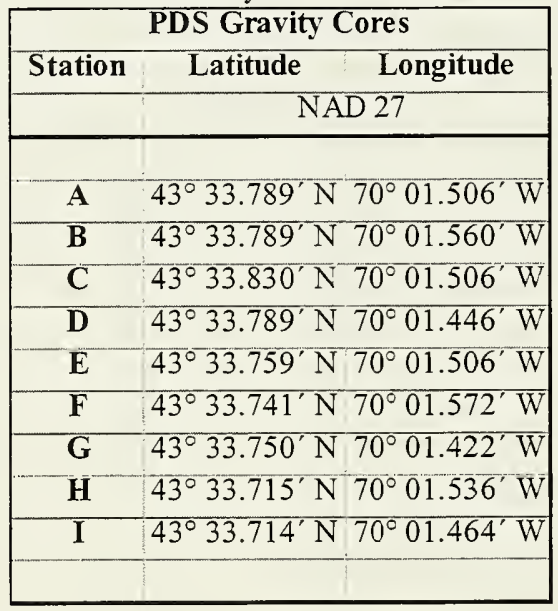

The SAIC gravity corer included core barrels consisting of a $1.5 \mathrm{~m}(5 \mathrm{ft})$ section of Schedule 40 PVC piping (10.2 cm or 4.0 ID) and included a stainless steel core cutter and core catcher at the end. Upon collection, the PVC tube was cut, plugged, and capped to prevent movement of sediments within the core during transport and storage. The postcap survey cores were transported back to the GSO laboratory facilities and refrigerated during storage.

At the GSO, the postcap sediment cores were split, visually described, and sampled for grain size, as well as for mineralogical and microfossil composition. A total of 92 samples ( $2.5 \mathrm{~cm}$ plugs) were taken from the nine cores and placed in pre-cleaned plastic containers. Forty-six samples were sealed and forwarded to an independent laboratory for grain size analysis. The remaining 46 samples were covered with a buffered formaldehyde solution for meiofauna preservation and sent to the micropaleontology labs for processing and analysis.

\subsection{Additional Royal River Cores}

\subsubsection{Selection and Processing of Additional Cores}

After the initial analysis of the Royal River cores, and the precap and postcap 
sediment samples, a data gap was identified between Cores RR-15 and RR-26 (Figures 2-4 and 2-9). Four additional cores from the Royal River were examined to clarify distinctions between the upper and middle reaches of the estuary. To better characterize the sediments in the boundary region between the two reaches of the river, Cores RR-6, RR-5, and RR-3 were selected from cores archived at the GSO and processed at the SAIC Environmental Testing Center in the summer of 1997. In addition, comparisons were made between samples preserved in methanol and formalin (Section 3.8.2).

The procedures for washing, sieving, and examining mineralogy and picking of microfossils in the $63 \mu \mathrm{m}$ to $500 \mu \mathrm{m}$ fraction were the same as described in the methods for the other core and grab samples (Section 3.7). Most of the gray, oxidized exterior of the core half was removed prior to sampling. Core RR-3 samples are split into two sections to compare formalin and methanol preservation solutions; the other two cores were placed in formalin solution only (Section 3.8.2). Given the depth actually dredged for each location, RR-6 sample was a composite of the top 1 foot of core; RR-5, of the top 4 feet; and RR-3, of the top 3 feet. The bottom section of RR-3 was not used because it was found to be dry and cracked. The second section of RR-5 from which the top 1 foot was sampled was slightly drier and more oxygenated than the other cores.

\subsubsection{Comparison of Methods of Microfossil Preservation}

The samples originally collected for microfossil analysis from the Royal River cores were stored in a $70 \%$ methanol/seawater solution. Because the density of microfossils was very low in some Royal River samples, a stronger fixative (buffered formalin with Rose Bengal stain) was used to compare relative preservation between the two methods. For the precap survey meiofauna and mineralogical analyses, the sediments of each grab sample were subdivided into two pre-cleaned 1 liter bottles, with one preserved in a $70 \%$ methanol/seawater solution, and one preserved in a buffered Rose Bengal/formalin/seawater solution. Both samples collected from grab Station 50NE were processed and analyzed for specimen concentration comparisons between the methanolbased versus the formalin-based preservatives.

The preliminary results showed that the dredged material preserved in the buffered Rose Bengal/formalin solution yielded a higher abundance of both foraminifera and thecamoebians relative to the methanol-preserved sample. As a result, only the sediments preserved in buffered Rose Bengal/formalin solution were analyzed for the remainder of the samples. For the postcap survey, all 46 samples were preserved with a buffered formalin solution for meiofauna preservation.

The final comparison of the effect of the type of preservative was conducted on samples collected from the additional Royal River cores (Section 3.8.1). Due to the paucity of microfossils in many of the sediment samples from the upper reach of the Royal 
River during the original analysis, one of the additional cores (RR-3) was split into two sections to evaluate the effect of the preservative. Results of this analysis are discussed in Section 4.6 in the context of the micropaleontological analysis results.

\subsection{Tracer Analyses}

\subsubsection{Sewage Tracer (Royal River only)}

Clostridium perfringens are microorganisms that naturally occur in the intestines of humans and animals. Because this species can survive for long periods of time in the environment, their presence in sediment may indicate fecal contamination that is several years old. On the Royal River (near RR-18), a sewage treatment plant releases treated effluent into the river between Wolfe's and Callen Points. Therefore, samples were analyzed for Clostridium perfringens to evaluate its potential as a tracer of the Royal River dredged material once deposited at PDS.

\subsubsection{Grain Size}

Grain size analyses were conducted using American Society for Testing and Materials (ASTM) Method D422-63 on grab samples collected during the precap survey, and cores collected during the postcap survey. Samples were sieved into size fractions greater than $62.5 \mu \mathrm{m}(<4 \mathrm{phi}$; sand and gravel), and less than or equal to $62.5 \mu \mathrm{m}(>4$ phi; silt and clay). The gravel and sand fractions were subdivided further by mechanically dry sieving it through a graded series of screens. The wet sieve and dry sieve fractions less than $62.5 \mu \mathrm{m}$ (silt and clay) were combined for each sample. The silt and clay fraction was then subdivided using a pipet technique depending upon differential settling rates of particles. Data on grain size were converted from units of phi to units of gravel, sand, silt, and clay (Wentworth 1922).

\subsubsection{Coarse Fraction}

All samples were sieved through a $0.5 \mathrm{~mm}$ screen $(>500 \mu \mathrm{m})$, dried, and weighed. For the Royal River samples, a general description of the primary component and secondary components if applicable, were recorded.

\subsubsection{Fine Fraction: Microfossil and Mineralogy Observations}

The samples were also sieved through a $0.063 \mathrm{~mm}$ screen. The fine fraction sediments $(<500 \mu \mathrm{m}$ and $>63 \mu \mathrm{m})$ were dried and weighed. Silts and clays $(<63 \mu \mathrm{m})$ were discarded. The fine fraction samples in this document is defined as the fine-medium sand component of the sediment. These were examined through the use of microscopy (magnification $40 \times$ to $100 \times$ ) to determine type and number of microfossils, as well as 
mineralogical composition. For this study, the term microfossil includes only foraminifera and thecamoebians, some of which may have been living meiofauna at the time of collection. Most microfossils are likely the shells of previously living organisms that have been preserved with the accumulation of sediment since the last dredging operation in the 1960's.

During the examination of the sediment samples for microfossils, observations of minerals and other constituents were also recorded to assist in future dredged material differentiation. Therefore, the term mineralogy also includes description of other microorganisms such as diatoms and ostracods for this project. The abundances of all parameters were noted and quantified, for statistical analysis of the mineralogy data, using the following scheme: $a b s e n t=0$, rare $=1$, common $=2$, and abundant $=3$.

The fine fraction material was randomly sub-sampled with a metal spatula and spread in a micropaleontology picking tray. Microfossils were picked until 100 specimens of foraminifera were collected, or ten full trays of material had been analyzed.

Foraminiferal and thecamoebian tests, as well as diatoms and ostracods, were mounted in cardboard slides with aluminum holders and glass cover slides and recorded. The grab and core samples collected at the PDS contained a higher abundance of foraminifera relative to the Royal River core samples. Therefore, microfossils were picked until 100 specimens were collected or five micropaleontology picking trays were examined for each sample. Following counting and identification of the microfossils, Scanning Electron Microscopic (SEM) photographs were taken of representative specimens (Figure 2-7).

For displaying and interpreting data, the microfossils were grouped into five categories based on a combination of factors: the identified informal group (foraminifera or thecamoebian), the ecological zonation (freshwater, mudflat, marsh, or continental shelf), and shell composition (agglutinated [silica] or calcareous) for foraminifera only. To determine the relative abundance of freshwater thecamoebians, marsh foraminifera, mudflat foraminifera, shelf agglutinated foraminifera, and shelf calcareous foraminifera, the number of individuals per category were divided by the total number of individuals for each sample. Relative abundance allows for comparison of samples despite differences in density or volume of material examined and picked. To determine the density of foraminifera and thecamoebians, the number of individuals were divided by the weight (grams) of material picked. Microfossil densities may be correlated with environmental conditions; for example, organic-rich silty clays may support larger populations than coarse sand areas with a limited food supply. Both relative abundance and density data were graphed, showing the position of the samples relative to the core.

\subsection{Multivariate Statistical Analyses of Fine Fraction Results}

The goal of the Royal River project was to define a tracer, or tracers, that would 
allow confident identification of dredged material layers from two areas of the same source area on the seafloor at the PDS. The fine fraction data were most promising as a diagnostic tool to allow differentiation of the layers at the Royal River Project capped mound. Because of the importance of this goal, fine fraction results from the cores collected during the postcap survey were statistically analyzed to evaluate the visual distinctions in the sediment layers of the Portland Capping Project disposal mound. Two overall methods of analysis were conducted on the sample dataset (Section 3.10.1). The first approach analyzed the entire dataset with no a priori classification of the material (Section 3.10.2). The second approach was to measure the strength of the classifications of postcap core samples into CDM, pseudo-UDM, and ambient units based on visual descriptions and microscopic analysis (Sections 3.10.3 and 3.10.4).

The techniques described below provided statistical data using a variety of approaches, and results were considered as a whole in this report. The tests were designed to address several questions/hypotheses. First, the analysis was necessary to compare the sample groupings assessed by the core visual descriptions in relation to the detailed microscopic analyses. Although the visual and sample data provide qualitative evidence for identifiable layers on the seafloor, these statistical analyses were able to address the significance of the differences between the CDM, pseudo-UDM, and ambient layers. Second, the relative correlation between the multiple variables, including biological, physical, and environmental factors, was assessed. The results are discussed further in the Discussion (Section 5.0).

\subsubsection{Sample Selection and Database Description}

Forty samples from seven of nine cores taken during the postcap coring survey were used for the analyses (Section 3.7.2). Data from cores H2 and F2, which were waterlogged and had unreliable stratigraphies, were excluded, as well as sample C1 (53-57 $\mathrm{cm}$ ) which contained only three microfossil individuals. For the mineralogy data, the abundances that were quantified prior to analysis (absent $=0$, rare $=1$, common $=2$, and abundant $=3$ ) were used for the statistical analyses described below. For the microfossil data analysis, the number of individuals of each species of foraminifera and thecamoebian were entered for each sample.

\subsubsection{Clustering and Multi-Dimensional Scaling Analysis}

Using PRIMER software (Plymouth Marine Laboratory, UK), two complimentary multivariate techniques were employed to assess the mineralogy and microfossil data: clustering and non-metric multi-dimensional scaling (MDS; Clark and Warwick 1994). Both of these tests were conducted on the sample database (Section 3.10.1) with no $a$ priori classification of the samples from the visual core descriptions. These tests are described briefly below, with references provided for further information. Following the 
MDS analysis, BIOENV was used to overlay specific environmental data over the MDS results to qualitatively evaluate other environmental factors contributing to the differences in the datasets (Clark and Warwick 1994).

Clustering independently determines the similarities between sample data points based on multiple variables, and then groups them accordingly. The cluster program standardized the data, so the relative abundance of each species was used for analysis. The abundance data were transformed by the fourth square root to minimize the dominance of the very abundant species, so the rare species also contributed to determining the similarity between samples. For each analysis, the Bray-Curtis similarity index was calculated and used to create a similarity matrix. Hierarchical agglomerate clustering with group-average linking was performed on the matrix for each dataset. The results were displayed in a dendrogram showing station groupings on the basis of Bray-Curtis similarity in the mineralogy composition or the microfossil community structure.

Non-metric MDS provides an ordination, or map, of samples showing the interrelationships between samples on a continuous scale. The MDS method compares the extent to which the data groups determined by clustering are similar. MDS ordination was performed on the similarity matrixes of the mineralogy data and the fourth square root transformed microfossil data as in clustering analysis. The plots were constructed by an iterative procedure, however, which successively refined the positions of the samples to reflect the similarity relations between them.

The BIOENV module of PRIMER was used to overlay various environmental datasets, including grain size data and microfossil densities (number of individuals per gram of picked material). These data groupings were overlaid on the MDS ordination plots. The additional variables were represented as symbols of differing size, determined by a simple linear function of the selected variable, and superimposed on the 2-dimensional MDS ordination of the microfossil data.

\subsubsection{Analysis of Similarities}

The samples collected in the cores following the postcap survey were classified as cap material (CDM), dredged material (pseudo-UDM), and ambient material, based on both the visual appearance (Section 3.7.2) and microfossil analysis (Section 3.9.4). The statistical strength of the differences between these pre-determined groups were evaluated using the ANOSIM (analysis of similarities) randomization test, applied to test for the statistical significance of differences displayed in the microfossil assemblages of the CDM and pseudo-UDM samples. ANOSIM is based on a non-parametric permutation procedure applied to the rank similarity matrix, described previously, which underlies the ordination of samples (Clarke and Warwick 1994). The procedure is analogous to standard parametric analysis of variance (ANOVA). 


\subsubsection{Discriminant Statistical Analysis}

The final statistical analysis was performed again with the data used to group the postcap samples into three pre-determined layers. Using SPSS ${ }^{\circledR}$ Professional Statistics 6.1, we performed a discriminant statistical analysis on the mineralogy and microfossil results from the core samples. Discriminant statistics is a multivariate program that identifies and forms linear combinations of independent variables which is then used to classify the samples into groups (Norusis 1994). Because the groups were pre-determined, the success of classification provides information on the differences between or similarities within the groups.

The mineralogy data were quantified the same way as previously described for the clustering analysis. In the SPSS package, the microfossil data were grouped into five categories that were used to display and interpret the data: freshwater thecamoebians, marsh foraminifera, mudflat foraminifera, shelf agglutinated foraminifera, and shelf calcareous foraminifera. The relative abundance of the five groups of species were calculated for individual samples. Each sample was then grouped as ambient, pseudoUDM, or CDM based on the depth of the sample with respect to the visual core descriptions and on microfossil content. The layer classification was the same as for the PRIMER statistical analyses.

The program calculated group means, standard deviation, and discriminant scores. To measure the degree of association between the scores and the groups, the discriminant scores were graphed according to two canonical discriminant functions. The canonical functions represent the ordination axes that best separate the known groups. The program determined the percentage of samples successfully classified into the pre-determined groups. 


\subsection{RESULTS}

To chronicle the events of the Portland Disposal Site Capping Demonstration Project, the results of the comprehensive field sampling and monitoring program are summarized in this section. The results of the five independent data collection efforts between August 1995 and February 1997 are presented below, including 1) the Royal River coring survey (Section 4.1); 2) the August 1995 baseline survey at the PDS (Section 4.2); 3) the expanded February 1996 baseline survey (Section 4.3); 4) the pseudo-UDM precap survey (Section 4.4); and 5) the CDM postcap survey (Section 4.5). In addition, results of the additional analysis of Royal River cores are presented (Section 4.6), and finally, results of the statistical analyses conducted on the analysis of all of the coring data (Section 4.7)

\subsection{Royal River Sediment Coring}

The coring survey conducted in the Royal River in August 1995 provided the information necessary to be able to track and differentiate sediments on the PDS seafloor, essential to the success of the Portland Disposal Site Capping Demonstration Project. The estuarine environment with distinct sediment characteristics in the brackish and marine reaches, the moderate volume of dredged material, and the location of the dredging area relative to PDS made the Royal River a strong candidate for the capping demonstration. The 30 collected cores (Figure 2-4) provided a cross section of the material to be dredged from the Royal River.

The sampled reaches of the river were classified to show the sediment characteristics of three estuarine zones (upper, middle, and outer) within the Royal River. A general description of the lithologies recovered in the cores is provided in Section 4.1.1, and then results from the more detailed analysis of the potential tracers evaluated from the cores is provided in Section 4.1.2. Core descriptions are provided in Appendix A.

\subsubsection{Sediment Characterization}

Several lithologic units were recovered from each of the three estuarine zones within Royal River. The upper zone of the river is in the transition area between freshwater and brackish environments that extends from the base of the falls to the eastern margin of the anchorage area. The material recovered from the upper zone, representative of the pseudo-UDM material to be dredged, consisted primarily of a subtidal to intertidal mudflat deposit. However, the material collected in Core RR-15 was very sandy, which may be attributable to past anthropogenic activity. Cores obtained from the middle and outer zones of the river, representing CDM, contained higher concentrations of sand, with the extreme outer cores (RR-21 and RR-22) containing layers of fine to medium sand. The outer region contained both sand flat deposits and flood/tidal channel deposits. Flood/tidal 
channel deposits are discrete units of organic debris, shell-rich layers, and sand/gravel layers that commonly occur in estuarine environments. These layers were commonly recovered in cores from all three of the Royal River zones. With natural deposit thicknesses ranging from $5 \mathrm{~m}$ to $20 \mathrm{~m}$ along the Maine coastline, these deposits tend to be poorly sorted and may contain larger organic and sedimentary debris.

The most commonly recovered lithologic unit, a subtidal to intertidal mudflat deposit, consisted of a dark greenish gray to black organic-rich clay to silty clay. At closer inspection, the unit varied from a highly detrital-rich unit in a clay matrix, to a siltier, more consolidated and homogenous unit with very finely disseminated organic debris. In general, the unit had fine bands of disseminated organic detritus (wood, sticks, leaves) throughout, and also contained discrete sand and gravel layers. In the upper zone of the river (anchorage area), the mudflat unit contained evidence for previous anthropogenic activity (slag and construction debris, Section 4.1.2.2).

The second most common lithology was a sand unit, recovered in many of the cores collected from the outer reaches of the river, as well as in the bottom of several cores collected in the middle to upper zones (Cores RR-1, RR-5, RR-14, and RR-16). The sand flat unit was a greenish gray to gray, silty sand to sand, again with organic detritus as in the mudflat unit. This unit was distinctive from the coarse, gravely sands that were documented in discrete units within many of the other cores (flood or tidal channel deposits). The sand flat deposit, dominant in the cores obtained from the mouth of Royal River, was typically light gray color, due to a higher shell fragment component (mussels, clams, oysters). In several cores, the fine sand unit was distinctly reflective from finely disseminated mussel shell fragments (RR-21, RR-22, RR-23, RR-29). The presence of thick sand throughout most of the cores obtained from the outer reaches of the river may also be part of a point bar sequence (recent sand deposit) present near the confluence of the Royal and Cousins River (MSPO 1983).

At the bottom of several cores (RR-12, RR-20, RR-21) there was a distinctive homogeneous gray clay that was characterized as stiff and well consolidated. This unit appears to be a glacially deposited fine clay, most likely from the Pleistocene Presumpscot Formation, which underlies the mud and sand flat deposits (Belknap et al. 1989).

\subsubsection{Sediment Tracer Analysis}

\subsubsection{Clostridium perfringens}

Clostridium perfringens are microorganisms that indicate the presence of fecal contamination, and were evaluated because of the location of a sewage treatment plant along the Royal River. Draft results of the Clostridium perfringens analyses indicated that the microorganism was present in all areas that were sampled, with the exception of the 
extreme western (RR-7) and eastern (RR-21) reaches of the area, and in predominantly sandy sediments. In fact, concentrations were often so high as to be above the upper screening unit. The presence of Clostridium in all of the areas suggested that there are other historical or modern sources of sewage (e.g., residential), or that the effluent from the sewage treatment plant is well mixed throughout the estuary. These preliminary results indicated that Clostridium would not provide a good tracer for the Portland Disposal Site Capping Demonstration Project, and therefore was not pursued.

\subsubsection{Coarse Fraction}

The most common components of the coarse fraction $(>500 \mu \mathrm{m})$ were wood, plant, and shell fragments, sand and gravel, as well as carbonized wood (wood that has been replaced by carbon in a reducing environment; Table 4-1). A material similar to the carbonized wood has also been described as charcoal, and attributed to the influx of burned material from burning and clearing of woodlands during colonial times (Belknap et al. 1989). The presence of a charcoal unit also may be a remnant of industrial activity; fragments of slag also were found in the coarse fraction samples. Preliminary analyses of the coarse fraction provided no distinctive material that was isolated to a specific reach of the river, and the most common components were present in most cores sampled. As a result, the coarse fraction data were not considered useful as a tracer.

\subsubsection{Fine Fraction}

Two major components of the fine fraction $(63 \mu \mathrm{m}$ to $500 \mu \mathrm{m})$ were analyzed: the mineralogical content, and the microfossil assemblages. As described in detail below, analysis of the microfossil content indicated differences in the composition of the microorganism populations; the presence and relative abundance of two informal groups of microorganisms, foraminifera and thecamoebia, varied in sediment originating from the different regions of the estuary. In addition, visual descriptions of the mineralogy of the sediments also showed distinctions in the material originating from the upper and outer reaches of the river. For both the mineralogical and microfossil data, the sediments from the middle zone had traits similar to both the outer and upper river zones, suggesting a blending of characteristics. As a result, we used both mineralogy and microfossils as tracers of Royal River dredged material.

Mineralogy. For the purposes of this report, the mineralogical composition of the fine fraction was defined as the mineralogical and biological components remaining after analysis of the microfossil (foraminifera and thecamoebia only) assemblages (Section 3.9). The fine fraction of all Royal River sediment cores were composed mainly of quartz and common micas (muscovite and biotite). The sediments also contained varying amounts of shell fragments, smooth ostracods, and black porous material. This material appeared to be small pieces of burnt organic matter, representative of coal or burnt wood. The 
sediment grains ( $>63 \mu \mathrm{m}$ - no clay/silt fraction) in the upper zone varied from very fine to medium gray sand, whereas the outer region displayed only coarse sand. As expected, the grain size in the middle zone varied from very fine to coarse sand.

The mean abundances for the mineralogical and biological components of sediment cores illustrated the traceable differences between the three zones of the river (Figure 4-1; Table 4-2). In Table 4-2, occurrence data are color-coded in order to show overall trends in each parameter. The mean abundance of all of the components ranged from none to rare in the upper reach, except for plant fragments that were rare to common in the upper and middle reaches and non-existent in the outer reach. Benthic and planktonic diatoms were present in the upper region only, but were rare ( $R R-8$ only). Only two components were present, but rare, in all three reaches of the river: black porous material and shell fragments.

\section{Table 4-1}

Core Fraction Results from the Royal River Survey

\begin{tabular}{|c|c|c|c|c|c|}
\hline & & & & Gen & Description \\
\hline $\begin{array}{c}\text { Core } \\
\text { Name }\end{array}$ & $\begin{array}{l}\text { Total } \\
\text { Length } \\
(\mathrm{cm})\end{array}$ & $\begin{array}{l}\text { Interval } \\
\text { Sampled } \\
\text { (cm) }\end{array}$ & Weight (g & Primary Component & Secondary Component \\
\hline RR-7 & I17 & $70-75$ & 1 & Organic debris (wood/plants) & Carbonized wood, sand \\
\hline RR-8 & 118 & $33-38$ & 24 & Wood fragments & \\
\hline $\mathrm{RR}-10$ & 189 & $142-147$ & 15 & Wood fragments & \\
\hline RR-12 & 195 & $20-25$ & 8 & Carbonized wood & Wood/plant fragments \\
\hline RR-15 & 98 & $22-27$ & 82 & Sand & Carbonized wood, small shell fragment \\
\hline RR-18 & 114 & $49-54$ & 1 & Wood/plant fragments & Shell fragments, carbonized wood \\
\hline RR-21 & 176 & $30-35$ & 43 & Sand, gravel & Shell fragments, (mussel and others) \\
\hline RR-22 & 194 & $45-50$ & 11 & Carbonized wood & Shell fragments, (mussel and others) \\
\hline RR-26 & 173 & $42-47$ & $<1$ & Shell fragments (mussel) & Carbonized wood, wood/plant fragment \\
\hline RR-28 & 176 & $20-25$ & 8.5 & Wood/plant fragments & Shell fragments, carbonized wood \\
\hline RR-29 & 194 & $35-40$ & $<1$ & Wood/plant fragments & Sand, shell fragments \\
\hline
\end{tabular}

Sand is predominantly well-rounded mineral fragments (quartz, metamorphic minerals).

The outer reach of the river had a common to abundant occurrence of fibrous minerals, with a rare to common occurrence of black porous material, flyash, molluscan shell fragments, and textured ostracods (Figure 4-1). Based on visual inspection only, the purple and white fibrous minerals were probably naturally occurring chrysotile asbestos. Bryozoan fragments were rare to common, and occurred only in the outer reaches of the Royal River. 
The middle reach shared characteristics of both the upper and outer river zones. Similar to the upper region, the middle samples contained plant fragments and fecal pellets. Similar to sediment cores at the mouth of the river, the middle region contained similar quantities of rock fragments and textured ostracods. However, in contrast to both the upper and outer zones of Royal River, the fine fraction samples for the middle zone contained a variety of insect parts, and no flyash.

Microfossils. The microfossils in the Royal River cores also served to differentiate the upper and outer zones of the Royal River. In general, the microfossil assemblage in the upper reach cores consisted primarily of mudflat foraminifera and thecamoebians. The number of individual thecamoebian species decreased with distance from the upper reach. Cores from the outer reach were dominated by mudflat and marsh foraminifera, while samples from the middle reach, as with mineralogical analyses, resulted in a combination of the two end member assemblages. Raw data from microfossil analyses is provided in Appendix B.

Samples from many of the cores collected in the upper reach resulted in a low overall abundance of microfossils counted in ten trays of material. For example, RR-7 contained only 3 freshwater thecamoebians, RR-8 contained 16 mudflat foraminifera and 27 thecamoebians (Figure 4-2), and sediment from RR-15 did not contain any microfossils, probably because it consisted of coarse sand which is inhospitable to foraminifera and thecamoebians. One of the additional upper reach cores analyzed, RR-6, had significantly higher numbers of microfossils, with 127 foraminifera and 25 thecamoebians picked in 3 trays (Section 4.6). The percentage of thecamoebians increased with the proximity of the core location to the upper river region. Core RR-6, analyzed as part of the additional core analysis (Section 4.6), was also different from the other upper reach cores in that shelf calcareous and agglutinated foraminifera specimens were present. One of the shelf agglutinated species in RR-6, Martinotiella communis, was not seen in any of the prior samples from the Royal River or at the PDS. The difference in microfossil abundance in RR-6 and the other additional cores are discussed further in Section 4.6. Shelf species were also noted in RR-18 and RR-26 (Appendix B); the trace appearance of shelf species has important implications in the interpretation of the cores collected from the dredged material deposit.

A lack of microfossils was also noted in some of the cores collected in the middle (RR-10, RR-29) and outer (RR-21) reaches. The low number of individuals counted tends to decrease the accuracy and reliability of the relative abundance calculations for those cores. As noted in Section 3.1.2, a methanol solution was used to preserve all microfossils samples from the sediment cores collected as part of the Royal River survey, and was shown to be less effective than the formalin preservative used for grab and core samples at the PDS site (Section 4.6). 


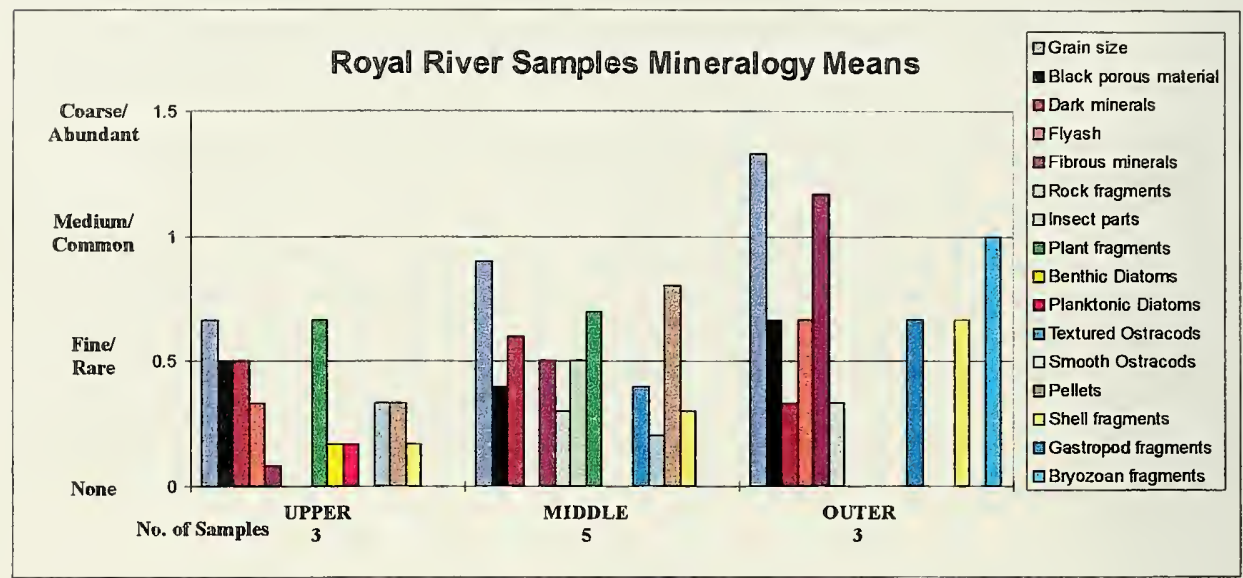

Figure 4-1. Histogram comparing mean abundance (rare, common, abundant) and particle size (fine, medium, coarse) for the mineralogical components utilized as sediment tracers from the Royal River cores 

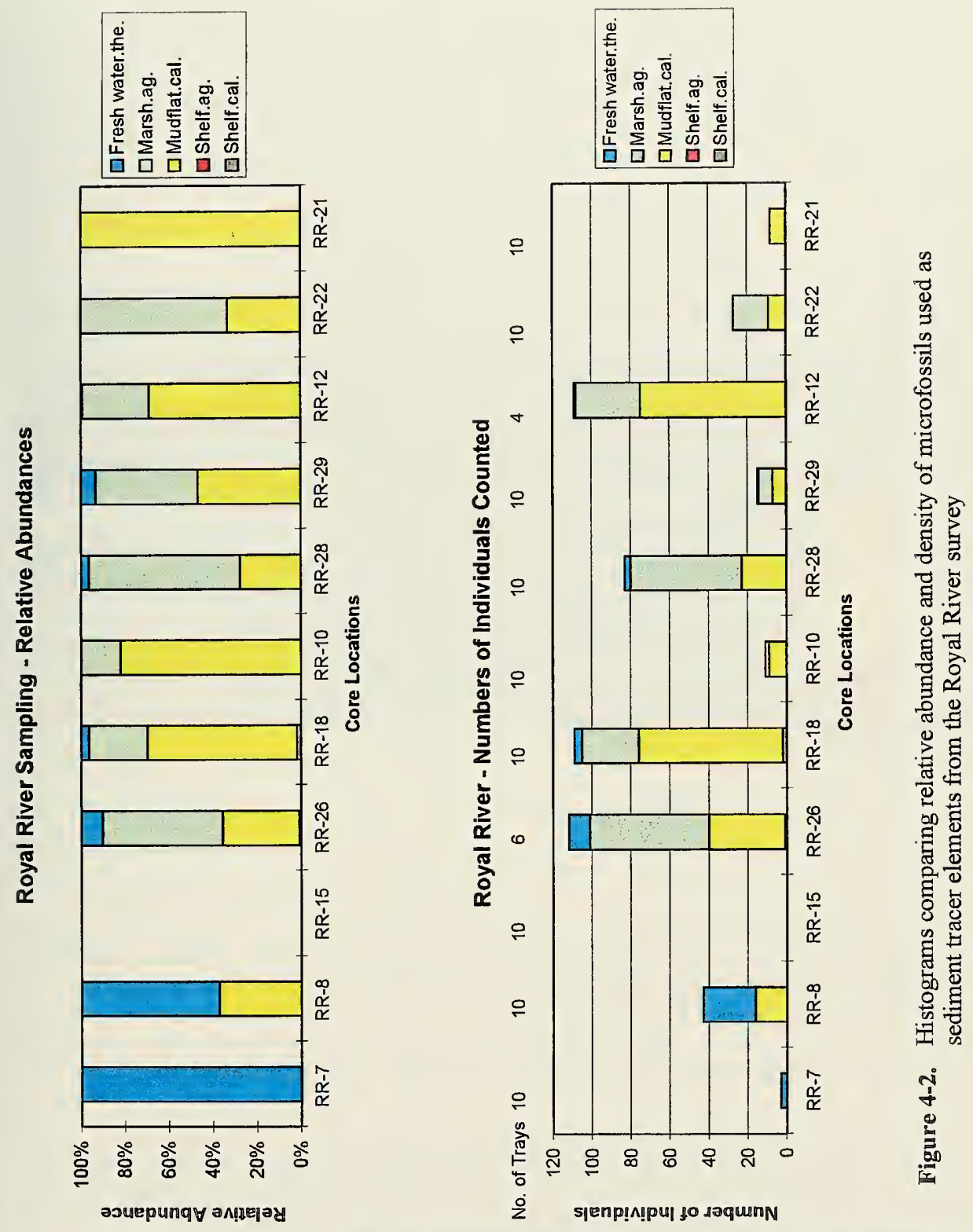
Table 4-2

\section{Royal River Core Sample Fine Fraction Mineralogy Results}

\begin{tabular}{|c|c|c|c|c|c|c|c|c|c|c|c|c|c|c|c|c|c|}
\hline 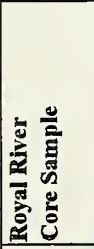 & 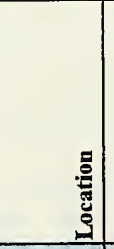 & 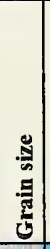 & 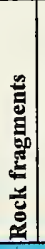 & 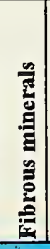 & 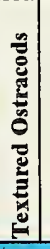 & 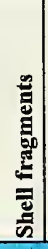 & 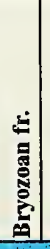 & 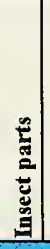 & 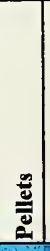 & 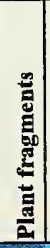 & 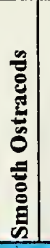 & 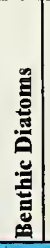 & 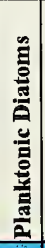 & 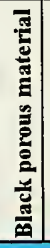 & 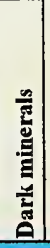 & 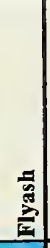 & Key \\
\hline RR-7 & UP & $\mathrm{F}$ & & & & & & & & $\mathrm{X}$ & & & 1 & $\therefore$ & $\therefore$ & -1 & Min. Parameter \\
\hline RR-8 & UP & F & & & & & & & $\mathrm{X}$ & $x$ & $x$ & $\mathrm{x}$ & $\mathrm{x}$ & $x$ & $x$ & $\mathrm{x}$ & Occurrence \\
\hline RR-15 & UP & $\mathrm{M}$ & & & & $x$ & & & & & & & & $\mathrm{X}$ & $\mathrm{x}$ & & XX Abundant \\
\hline RR-6 & Add UP & $M$ & $\bar{X}$ & & $\bar{x}$ & $\bar{x}$ & & $\mathrm{x}$ & $\mathrm{x}$ & $\mathrm{X}$ & & & $x$ & $\mathrm{X}$ & $\bar{x}$ & $\mathrm{x}$ & \begin{tabular}{|l|}
$\mathrm{X}$ Common \\
\end{tabular} \\
\hline RR-5 & AddU/M & M & $\mathrm{x}$ & & & $x$ & & $\mathrm{x}$ & $x$ & $x x$ & & & $x$ & $x \times$ & $\mathrm{x}$ & $\mathrm{x}$ & $\mathbf{x}$ Rare \\
\hline RR-3 F & AddMID & M & $\mathrm{x}$ & & & & & $\mathrm{x}$ & $\mathrm{x}$ & $\mathrm{x}$ & & & $\mathrm{x}$ & $\mathrm{X}$ & $\mathrm{x}$ & $x$ & Absent \\
\hline RR-3 M & AddMID & $\mathrm{M}$ & $x$ & & & & & $x$ & $x$ & $x$ & & & $x$ & $\mathrm{X}$ & $x$ & $x$ & Grain size \\
\hline RR-26 & MID & $\mathrm{F}$ & & $\mathrm{x}$ & $x$ & & & $x$ & $\mathrm{X}$ & $X$ & $x$ & & & $\bar{x}$ & $\mathrm{x}$ & & C. Coarse \\
\hline RR-18 & $\mathrm{MID}$ & $\mathrm{F}$ & & $x$ & & & & $\mathrm{x}$ & $\mathrm{X}$ & $x$ & & & & $x$ & $x$ & & M Medium \\
\hline RR-10 & $\mathrm{MID}$ & C. & $\mathrm{X}$ & $\mathrm{x}$ & & $\mathrm{x}$ & & & & $\mathrm{x}$ & & & & & $x$ & & F Fine \\
\hline RR-28 & $\mathrm{MID}$ & $\mathrm{M}$ & & $x$ & $x$ & $\mathrm{x}$ & & & $x$ & & & & & $\mathrm{X}$ & $x$ & & Dredged Sediments \\
\hline RR-29 & MII & $\mathrm{M}$ & $\mathrm{x}$ & $x$ & & $x$ & & $x$ & $\mathrm{X}$ & $\mathrm{x}$ & & & & & $x$ & & Disposal Destination \\
\hline RR-12 & OUT & $\mathrm{M}$ & & $\mathrm{X}$ & & $\bar{x}$ & $x$ & & & & & & & $\mathrm{X}$ & & $\mathrm{X}$ & PDA 96 UDM \\
\hline$R R-22$ & ourt & & & $\mathrm{x}$ & 4 & $x$ & $x$ & & & & & & & $x$ & & $x$ & PDA 96 CDM \\
\hline RR-21 & OUT] & $\mathrm{CH}$ & $\mathrm{X}$ & $\mathrm{xx}$ & $\mathrm{x}$ & $\mathrm{X}$ & $\mathrm{x}$ & & & & & & & & $\mathrm{X}$ & & DG Buoy \\
\hline
\end{tabular}

Add $=$ Refers to additional core analyses.

In contrast to the lack of marsh and mudflat foraminifera in the upper reach, these microfossils were abundant in the outer reach of the river, with only one thecamoebian found in Core RR-12. The middle zone of the estuary had variable population sizes, with marsh and mudflat foraminifera predominant. The cores collected from the middle zone of Royal River contained relatively fewer thecamoebians and a rare number of shelf foraminifera.

Because thecamoebians and mudflat/marsh foraminifera are all benthic species that live on or in the substrate, transportation of the microfossils is likely to be limited in the Royal River. Many of the calcareous foraminifera had protoplasm remains on their shells, 
and therefore possibly were alive at the time of collection. Oxidation of the organic matrix of foraminifera or thecamoebian shells will break apart the protein which holds the shell together. In addition, agglutinated shells are not strong and do not maintain their integrity long when subjected to mechanical transport. The state of naturally preserved foraminiferal tests and ostracods (mineralogy) suggested that many individuals were collected near their habitat range.

\subsection{Royal River Project Area August 1995 Baseline Survey}

The baseline survey conducted in August 1995, an $800 \mathrm{~m} \times 800 \mathrm{~m}$ bathymetric survey over the Royal River Project Area at PDS, was designed to serve as the basis for comparison with all future investigations. The processed data yielded a chart of the complex bottom topography in the southeast corner of the disposal site (Figure 4-3). Depths ranged from $48 \mathrm{~m}$ along the northern survey margin to $72 \mathrm{~m}$ in the southwest region of this $0.64 \mathrm{~km}^{2}$ survey area. A northwest-southeast trending trough, with an average depth of $62 \mathrm{~m}$, was selected as a suitable site to conduct the capping experiment. The moderate relief at this site would maximize the potential for detecting bathymetric changes and minimize the presence of survey artifacts common in regions with a rough, irregular topography. In addition, a subtle basin feature within the trough was determined to be advantageous for the development of a stable dredged material disposal mound (Figure 4-3).

The baseline bathymetric data were used to design the monitoring plan for the capping project. The ridges surrounding the basin were predicted to serve as natural containment measures, restricting the lateral spread of the dredged material disposal mound (Figure 4-4). However, a narrow depression in the seafloor to the south of the disposal basin was identified as a potential route for the downslope transport of sediments upon initial deposition. In the event that dredged material was deposited greater than $100 \mathrm{~m}$ southeast of the PDA buoy, or the angle of repose for the sediments composing the southern flank of the disposal mound (estimated to be $4^{\circ}$ ) was exceeded, small volumes of unconsolidated silts and clays were predicted to spread laterally through this passage. To document the possibility of an expanded pseudo-UDM mound apron, the REMOTS ${ }^{\circledR}$ sediment-profile photographic survey grid was designed to track the dredged material by concentrating sampling efforts over likely paths of dredged material transport (Section 4.3.2).

\subsection{Expanded Royal River Project Area February 1996 Baseline Survey}

The bathymetry of the Royal River Project Area was re-surveyed because the placement of $2,800 \mathrm{~m}^{3}$ of dredged material from the Harraseeket River at the project area (Section 2.1) potentially would confound future interpretation of sequential bathymetric surveys. In addition to the second baseline bathymetric survey, side-scan sonar and 


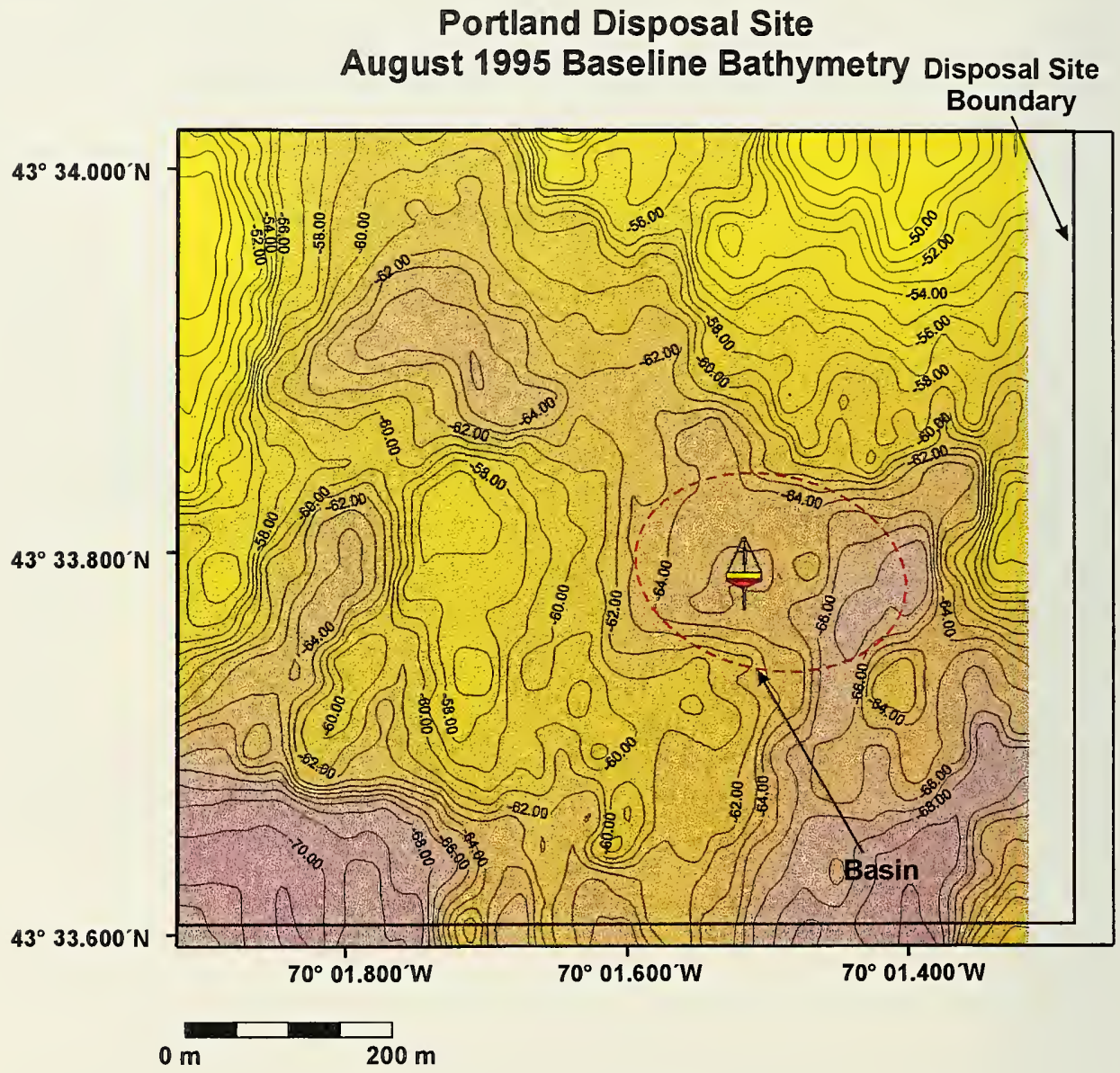

Figure 4-3. Bathymetric chart of the August $1995800 \mathrm{~m} \times 800 \mathrm{~m}$ Royal River Project Area (NAD 27) relative to the disposal site boundaries, $1.0 \mathrm{~m}$ contour interval (MLLW) 


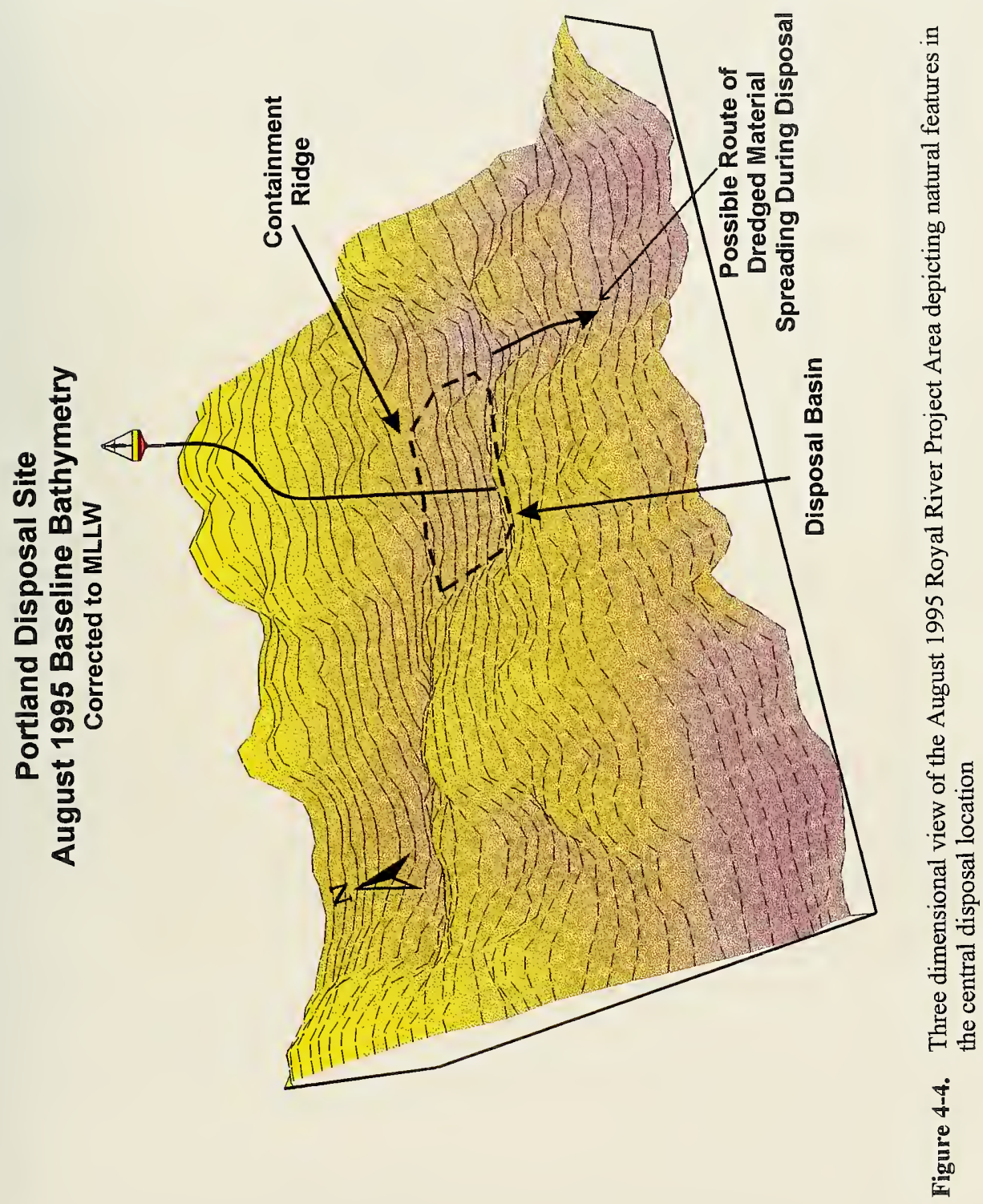


sediment-profile camera data also were collected in February 1996. The precision bathymetric and side-scan sonar surveys were performed over an expanded $1950 \mathrm{~m} \times$ $1000 \mathrm{~m}$ survey area in the southern region of the disposal site. The additional survey area was added to aid in establishing a deployment location for a physical oceanographic instrument array (McDowell and Pace 1997). Sediment-profile and planview photographs were collected within the project area to track the newly deposited material and provide photographic baseline data of the project area.

\subsubsection{Bathymetry}

The results of the $1950 \mathrm{~m} \times 1000 \mathrm{~m}$ bathymetric survey revealed an irregular topography to the west of the Royal River Project Area (Figure 4-5). A significant difference in depth (approximately $30 \mathrm{~m}$ ) is noticeable between the northwest and southeast corners of the survey area. At a contour interval of $1 \mathrm{~m}$, the steep slopes and rough topography throughout the $1.95 \mathrm{~km}^{2}$ area were clearly visible, suggesting the presence of exposed bedrock over much of the PDS seafloor (Figures 4-6). The February 1996 bathymetric data were re-gridded to the smaller $800 \mathrm{~m} \times 800 \mathrm{~m}$ project area, improving resolution for comparisons with the August 1995 dataset. Not unexpectedly, depth difference calculations between the two surveys were not able to resolve accurately the small volume $\left(2,800 \mathrm{~m}^{3}\right)$ of dredged material from the Harraseeket River. Further surveys using photographic technologies did reveal the presence of fresh dredged material (Section 4.3.2).

\subsubsection{REMOTS ${ }^{\circledR}$ Sediment-Profile Photography}

SAIC collected three replicate photographs from a total of 24 stations within the REMOTS $^{\circledR}$ sampling grid during the February 1996 field effort (Figure 4-7). Evidence of dredged material deposition was seen primarily in the REMOTS ${ }^{\circledR}$ photographs collected northwest of the buoy location. The character of the dredged material varied from "fresh" looking low optical reflectance fine-grained clay attributed to Harraseekt River material (Figure 4-8), to relic dredged material with a higher reflectance, attributed to older, historical dredged material.

The recently deposited Harraseeket River material, consisting of a thin layer of dark silt and clay, was seen within $100 \mathrm{~m}$ northwest of the PDA buoy position (Figure 4-7). In several replicates, a relic redox potential discontinuity (RPD) layer (the depth of oxygenation in the upper sediment strata) was visible below a newly formed oxygenated layer at the sediment/water interface. The presence of a relic RPD is indicative of recent sediment deposition (Figure 4-8).

Dredged material thicknesses greater than camera penetration depth were detected to the northwest, more than $100 \mathrm{~m}$ away from the center of the survey grid. This suggested 


\section{Portland Disposal Site Expanded Survey Area Bathymetry}

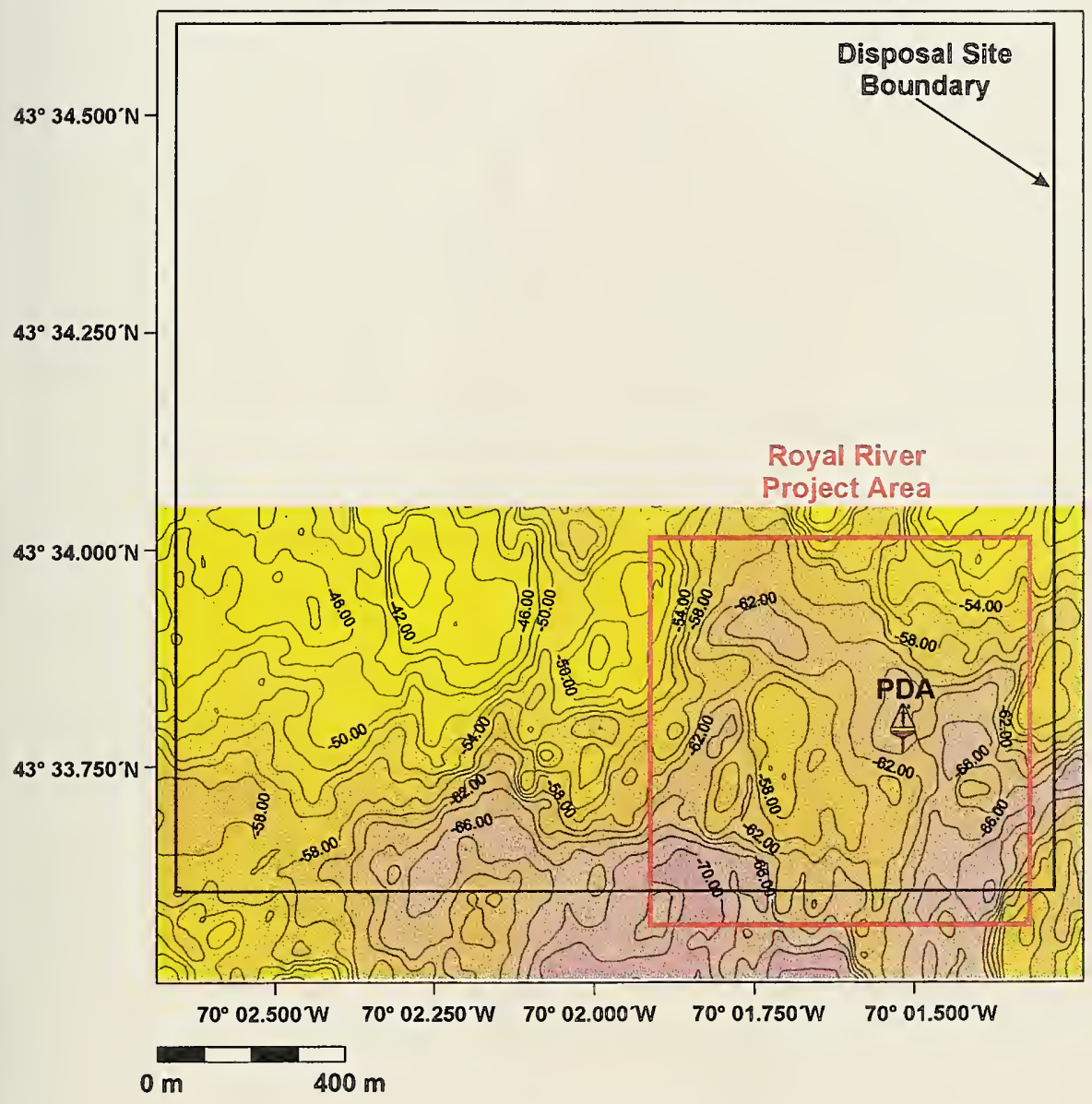

Figure 4-5. Bathymetric chart of the February $19961950 \mathrm{~m} \times 1000 \mathrm{~m}$ survey area (NAD 27) over the southern region of Portland Disposal Site relative to the project area and disposal area boundary, $2.0 \mathrm{~m}$ contour interval (MLLW) 


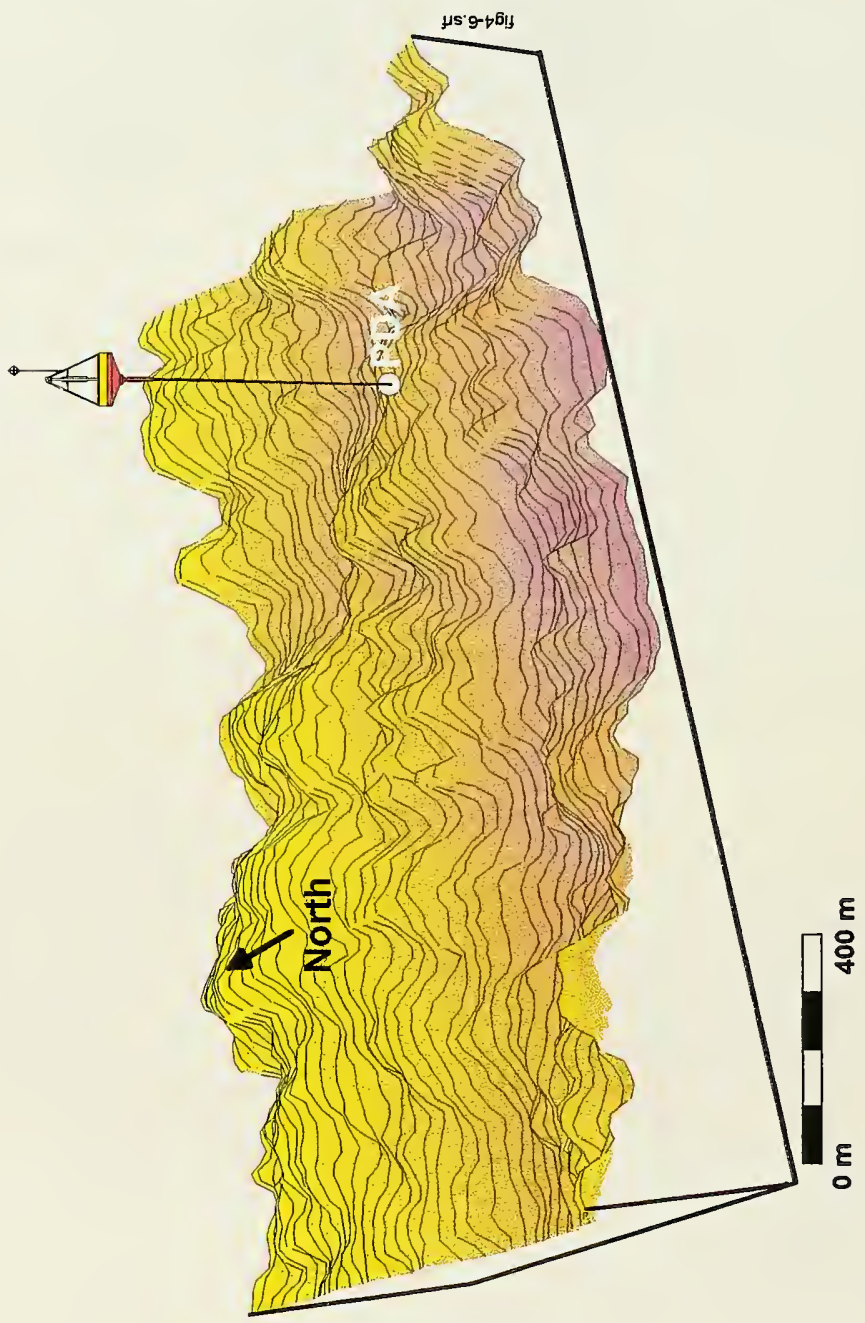


February 1996 Baseline REMOTS®

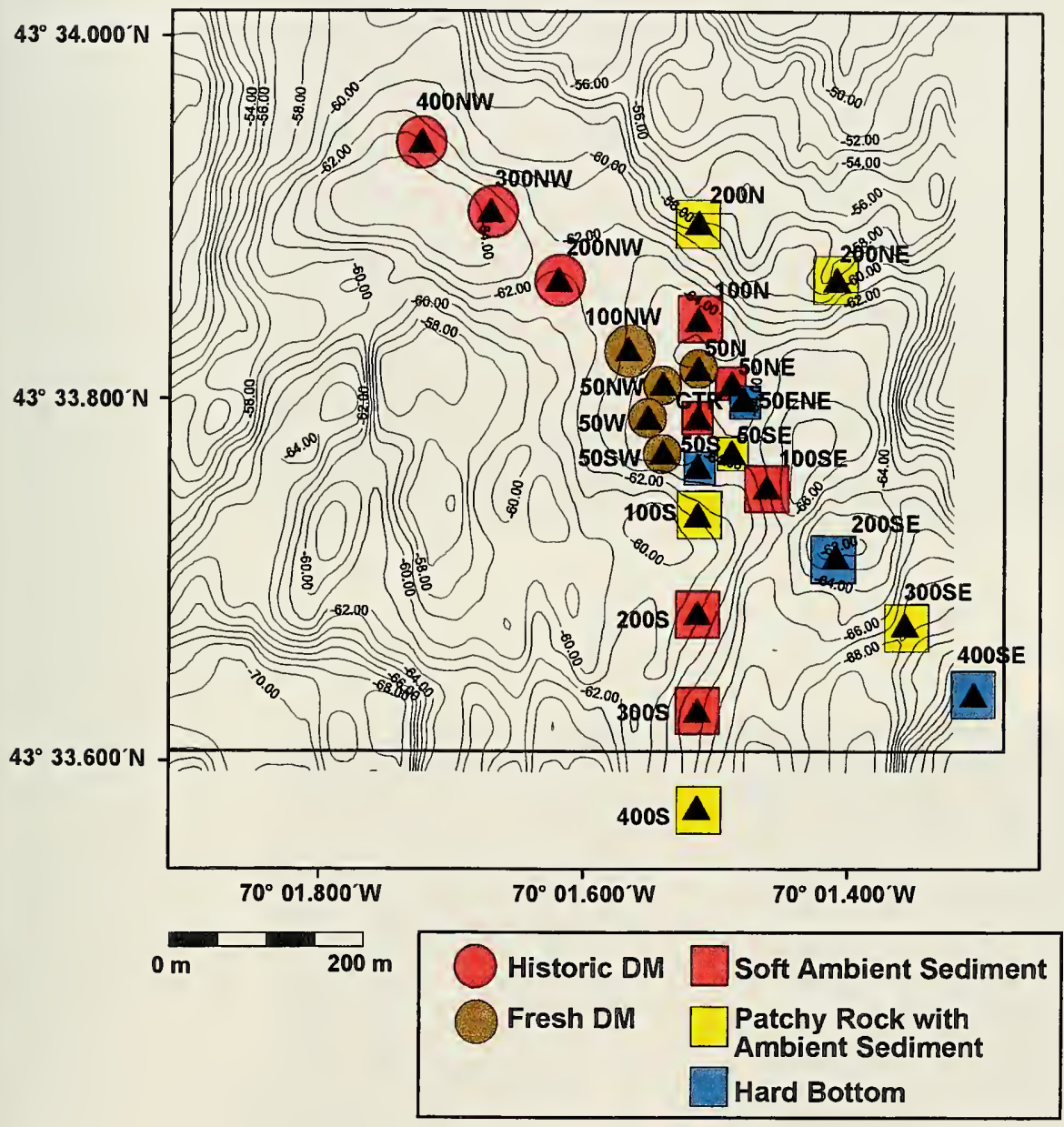

Figure 4-7. Characterization of ambient sediments and dredged material within the Royal River Project Area, as detected by the February 1996 REMOTS $\&$ camera survey 

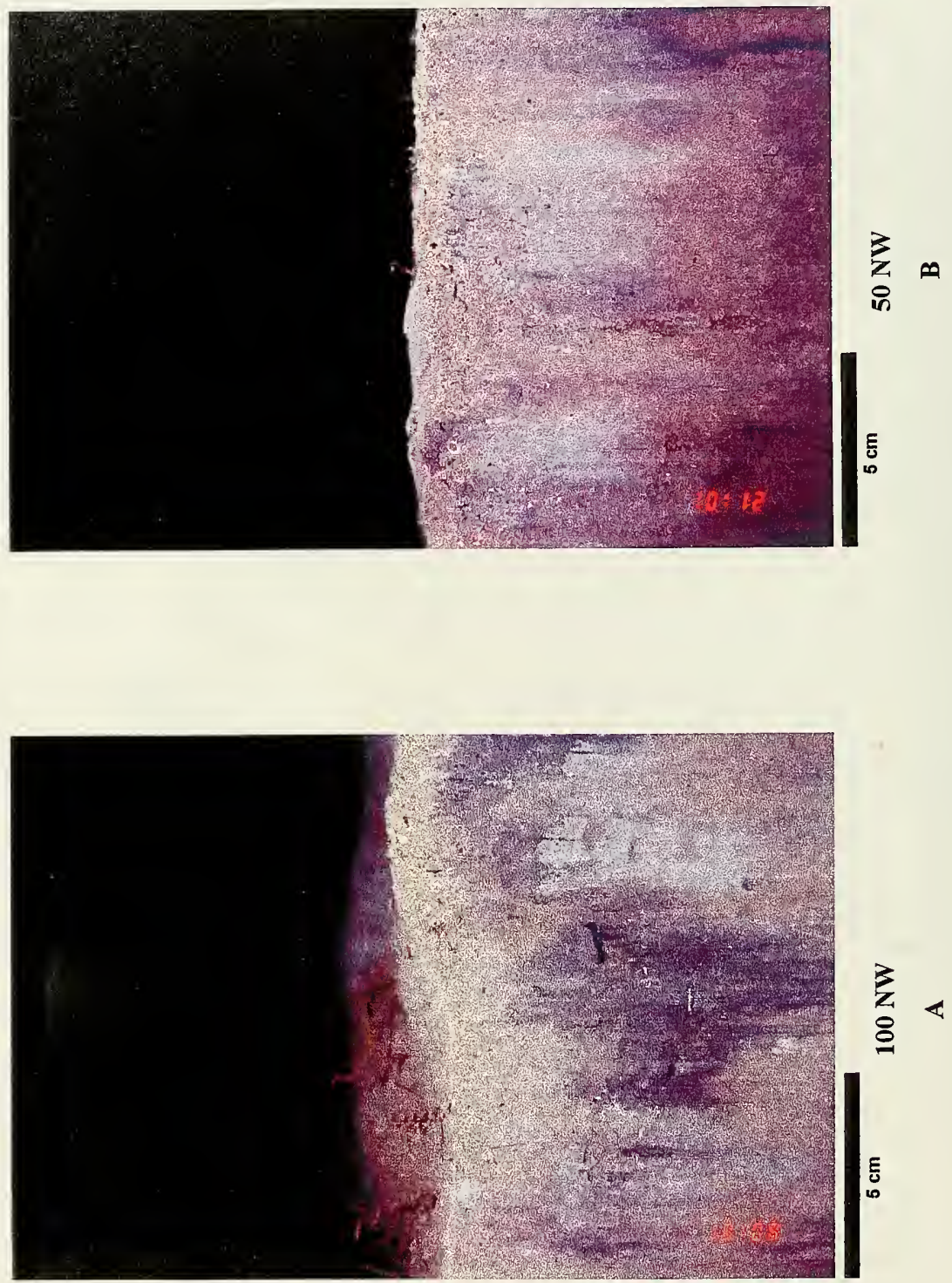

รั

on

寻壳

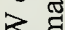

$\sum$

은?

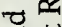

¿

3 ํㅝㅇ

乙

8 ․ㅗㅇ

犎

능

음

吾

क 호

명. 일

논

릉

ป

릉

8

(2)

등 응

का 0

옹

on

․․ㅁํㅁ

(a)

穴

ש2

잉

$\sum_{1}$

至

$\infty$
$\dot{1}$
0
0
0 
the presence of an apron of historical dredged material extending southeast from the 1990 1991 position of the DG buoy $\left(43^{\circ} 34.100^{\prime} \mathrm{N}, 70^{\circ} 01.900^{\prime} \mathrm{W}\right)$ into the Royal River Project Area, consistent with DAMOS disposal logs. The margin of the relic material was sampled at Stations 400NW, 300NW, and 200NW.

Ambient sediment, consisting of medium- to fine-grained tan sands commonly intermixed with cobbles, was detected at many of the stations on the southern and southeastern arms of the survey grid, as well as Stations 200N and 200NE (Figure 4-7). Tan sand with traces of reduced silt (possible relic dredged material) was found at Stations 50NE, CTR, and 50SW.

\subsubsection{Planview Photographs}

The planview camera provides information on the character of the surface of the seafloor. Photographs were collected within the northwest-southeast trending trough of the survey area (Stations 400NW - 400SE; Figure 4-7). All but two stations had a smooth silty seafloor in at least part of the camera's field of view. Heading southeast along this transect, the photographs from $400 \mathrm{NW}$ and $300 \mathrm{NW}$ showed smooth fine-grained sediments. Shrimp, shells, and a lobster pot trawl line were detected at $200 \mathrm{NW}$. Sediment-profile photographs indicated fresh dredged material at Station 100NW, corresponding planview images displayed clam and mussel shell fragments partially buried in the newly deposited silt (Figure 4-9A). Photographs collected over CTR displayed the same types of shell fragments, as well as worm tubes, large burrowing anemones, and a lobster (Figure 4-9B).

Turbidity caused by the touchdown of the camera baseframe before the acquisition of a planview image at $50 \mathrm{NW}$ prevented the collection of a clear image. The corresponding sediment-profile image indicated the presence of a silt and sand at the sediment/water interface. Scattered cobble to boulder size rocks appeared at Stations 50SE and 100SE (Figure 4-9C). A boulder sized rock at Station 300SE caused the REMOTS ${ }^{\circledR}$ camera frame to lay on its side. The resulting planview photograph showed a very irregular silt-covered rock outcrop. The last station in the transect planview photograph, from $400 S E$, showed a smooth seafloor with scattered rocks.

\subsubsection{Side-Scan Sonar}

In conjunction with the February 1996 bathymetric survey operations, SAIC collected side-scan sonar data over the southern region of PDS. Originally obtained to assist in the placement of a bottom-mounted instrument array for physical oceanographic studies (McDowell and Pace 1997), the acoustic images provided insight into the geology and topographic features within the $1.95 \mathrm{~km}^{2}$ area. The side-scan returns illustrated 

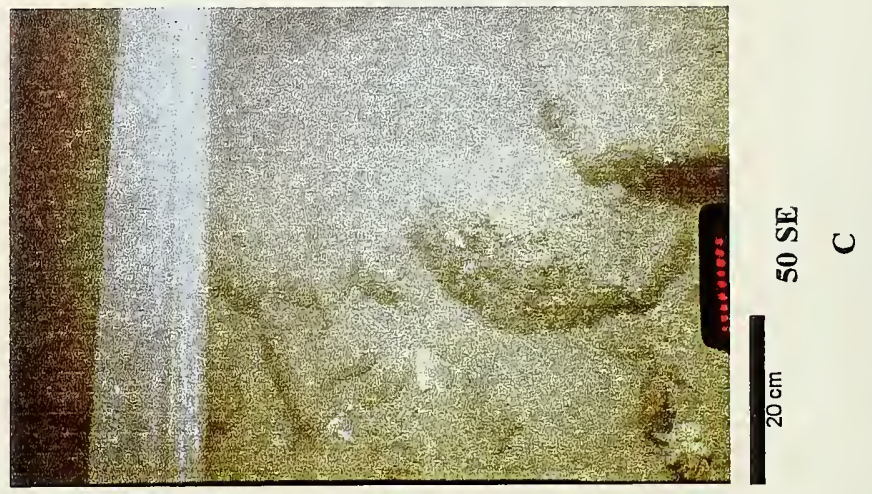

过
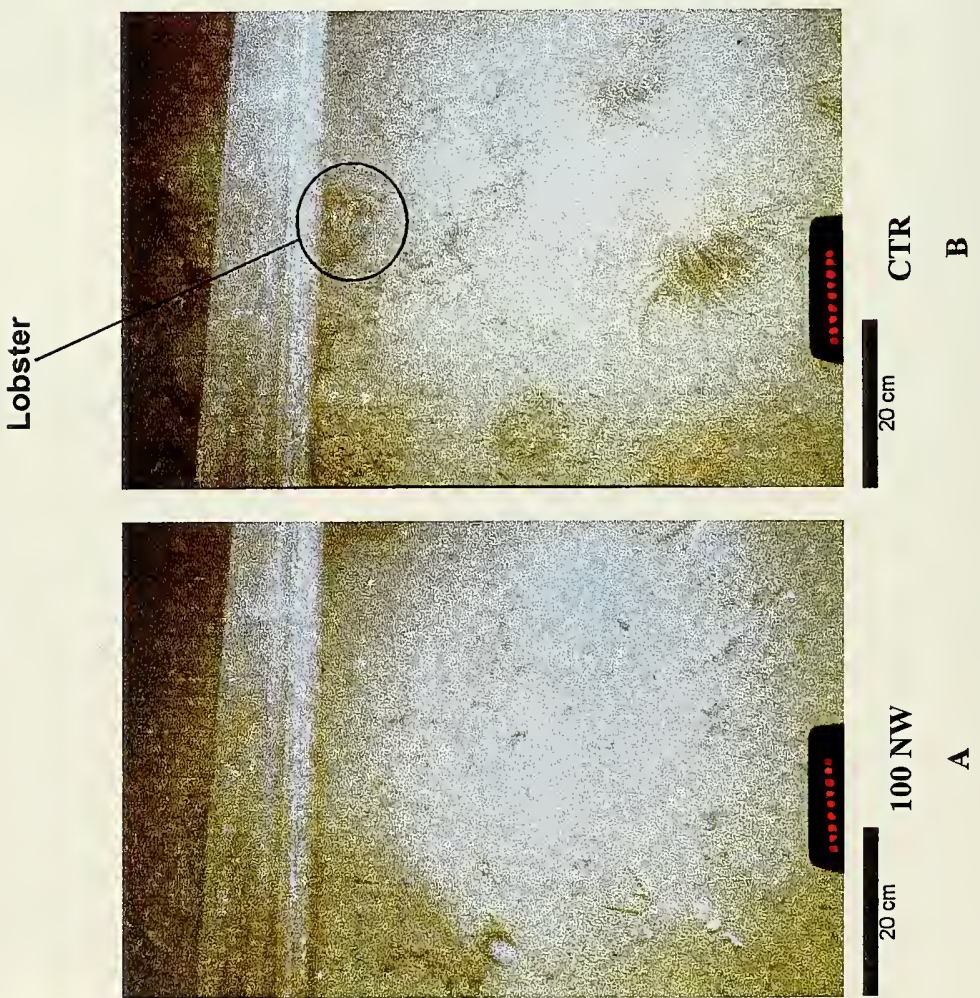

क्व

닝 तี

유융

$7=$

तै तี

芦

0

उ

番

乙오

을 를

겸

을

范

द्व ㅇํㅇ

H

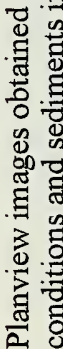

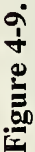


bedrock outcrops, large boulders, and sediment-laden valleys on the seafloor (Figure 410). Combined with the February 1996 bathymetric data, the side-scan sonar data depicted the geologic and sedimentological features of the disposal site (Figure 4-11). The data provided supporting evidence that the large basin feature identified in the bathymetric data would be the optimum area for receiving dredged material.

\subsection{Royal River Project Area Precap Survey}

Excavation of the anchorage area and upper channel reaches was conducted from 1 October 1996 to 14 November 1996 (Section 2.1). Disposal logs indicated an estimated barge volume of $39,500 \mathrm{~m}^{3}$ of pseudo-UDM was deposited to the south and southeast of the PDA buoy. The precap (pseudo-UDM) survey, consisting of precision bathymetry, REMOTS $^{\circledR}$ sediment-profile photography, and sediment grab sampling, was completed in mid-November to determine the height, size, and shape of the pseudo-UDM deposit, as well as track the distribution of dredged material within the project area.

\subsubsection{Bathymetry}

Following the deposition of pseudo-UDM, SAIC performed a third bathymetric survey over the $800 \times 800 \mathrm{~m}$ Royal River Project Area on November 20, 1996. Although no significant changes were clearly evident in the standard bathymetric chart generated for the project area, depth difference comparisons between the February 1996 baseline and the November 1996 precap survey did detect an accumulation of material to the south and southeast of the PDA buoy position (Figure 4-12). As predicted, survey artifacts occurred in the depth difference plots in regions with a highly irregular topography, corresponding to the strong slopes of bedrock outcrops to the south and west documented by the side-scan sonar results. Because in many areas the apparent thickness due to survey artifacts was greater than the accumulation around the PDA buoy $(>1 \mathrm{~m})$, a definitive description of the UDM material footprint required integrating both the bathymetric and sediment-profile camera data (Section 4.4.2).

The accumulation of pseudo-UDM tended to follow the confines of the PDS bottom topography with the detectable footprint of the mound remaining within the naturally occurring basin feature (Figure 4-13). The bathymetric profile of the UDM deposit showed two east-west oriented lobes of material approximately $1.25 \mathrm{~m}$ high. These apparent lobes, however, may have been compromised by survey artifacts. After analysis of sediment-profile data (Section 4.4.2), the eastern lobe, present over a pre-existing topographic feature, was likely a result of survey artifact. 


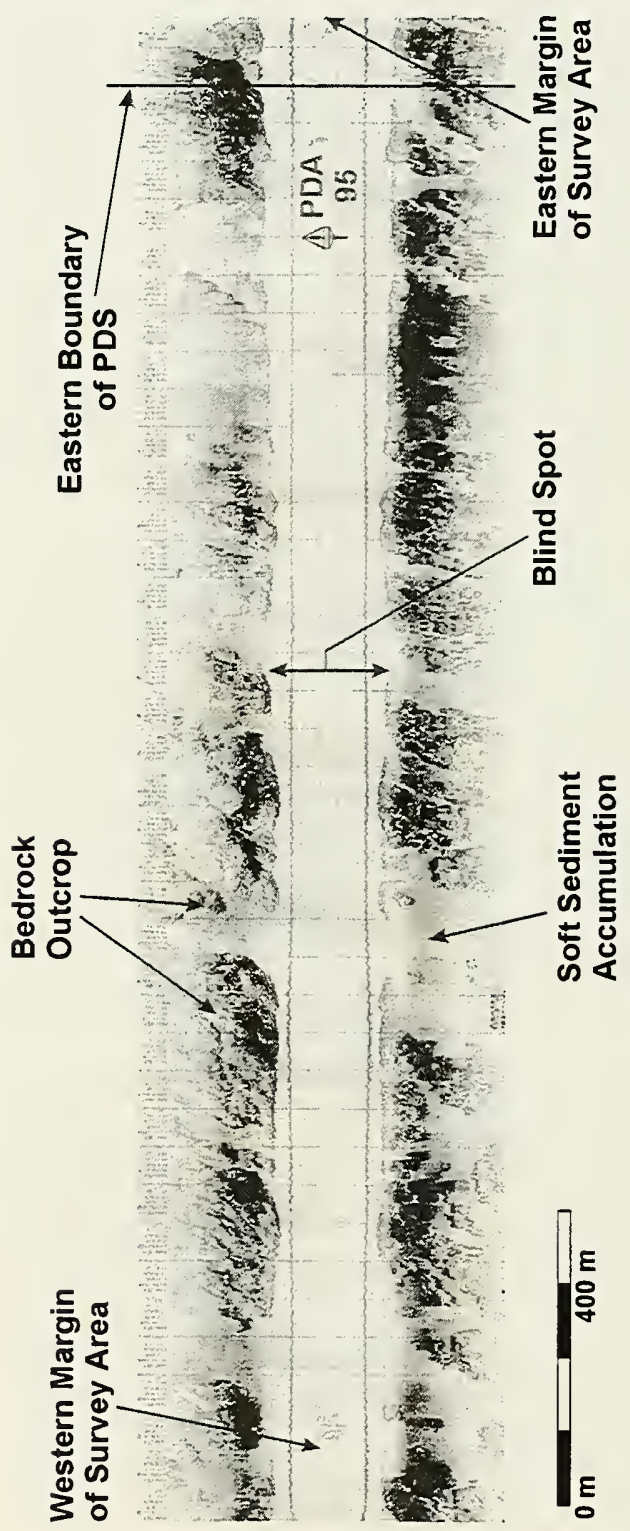



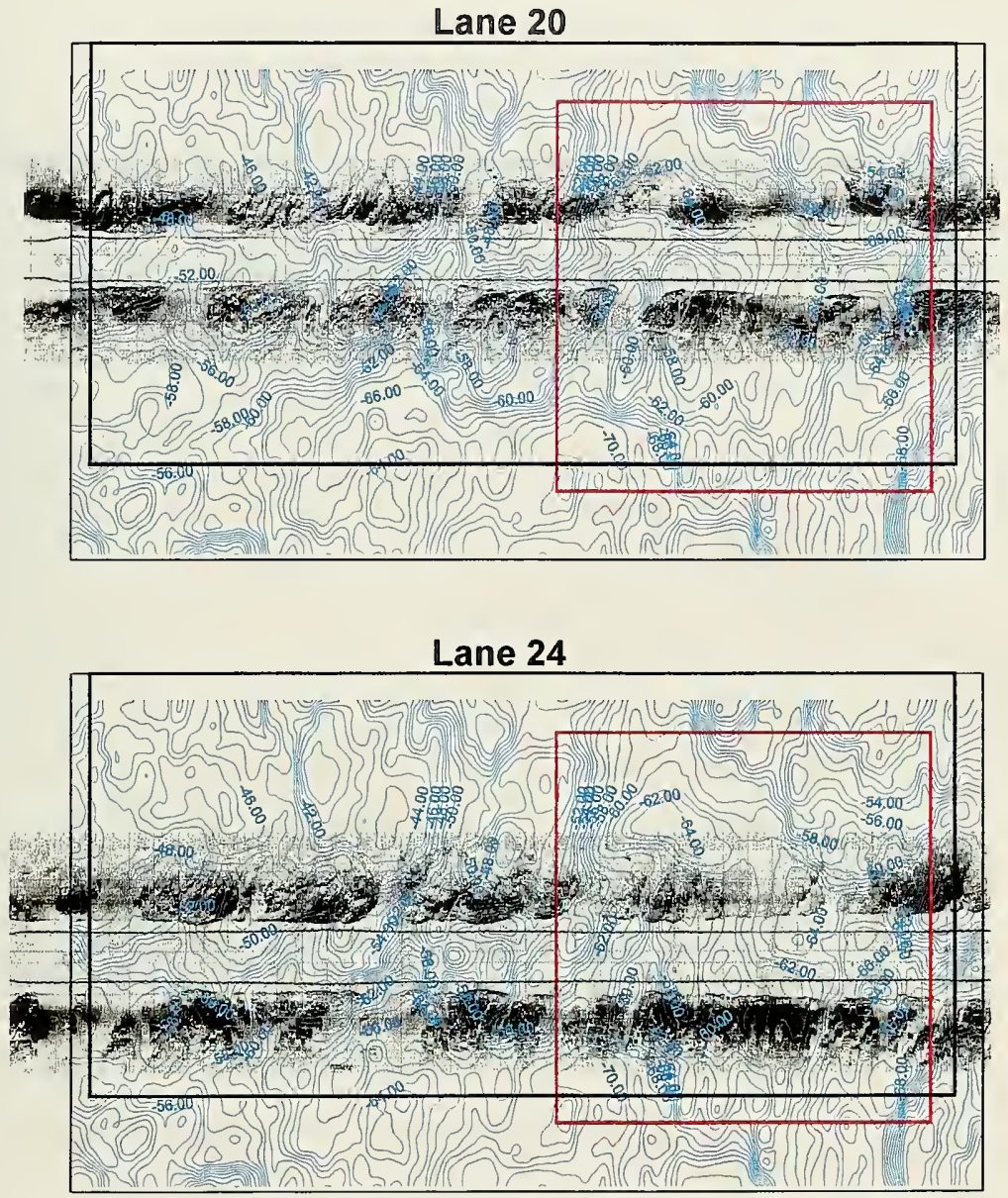

Figure 4-11. Side-scan sonar data from February 1996 survey lanes 20 and 24 overlaid with bathymetry showing rapid changes in depth due to bedrock outcrops 


\section{Portland Disposal Site \\ Royal River Project Area \\ Depth Difference}

February 1996 Baseline vs. November 1996 UDM Survey Apparent Accumulation of Pseudo-UDM

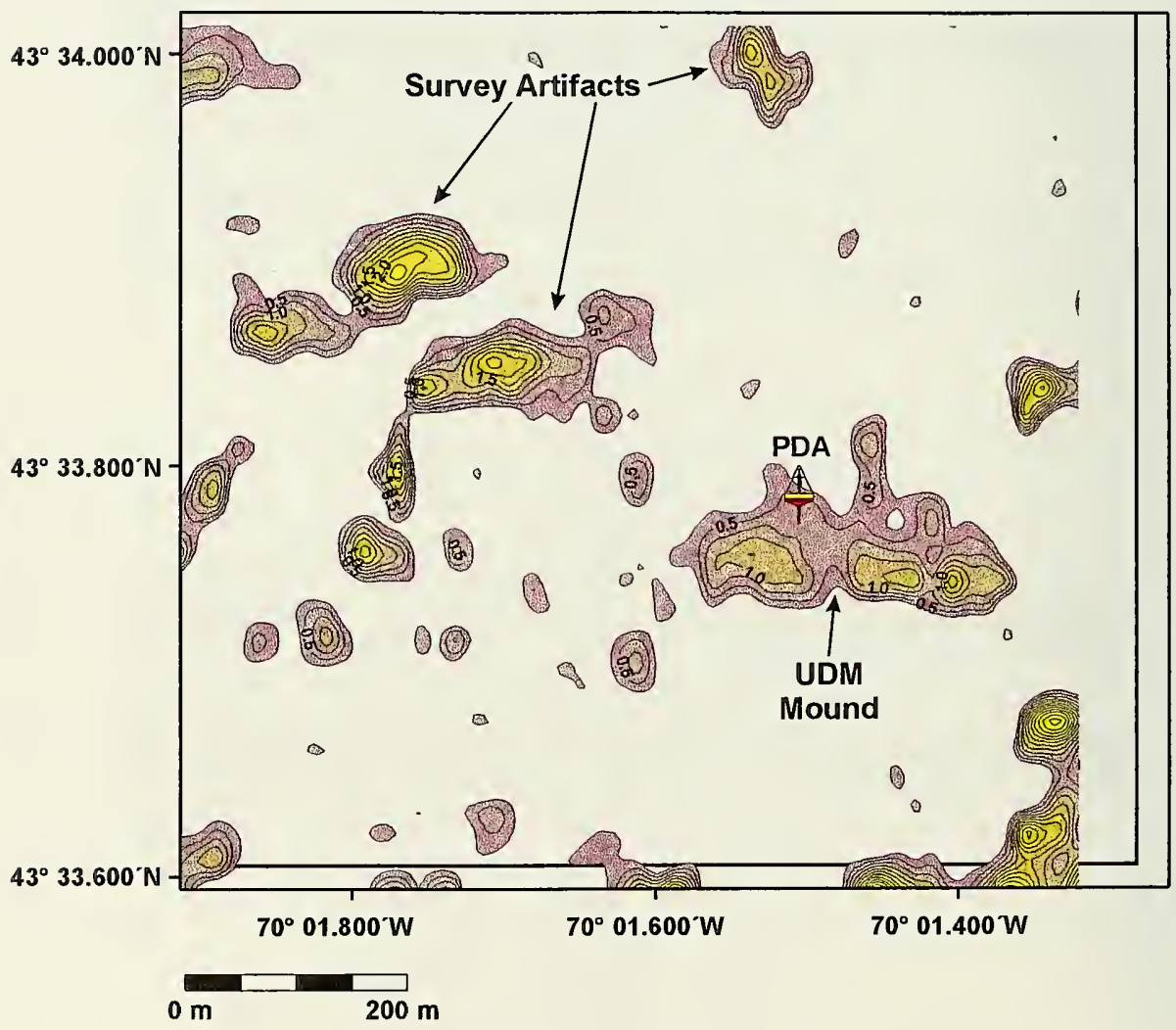

Figure 4-12. Depth difference plot of the November 1996 UDM survey versus the February 1996 baseline survey (NAD 27) showing apparent accumulation of pseudoUDM in close proximity to the PDA buoy, $0.25 \mathrm{~m}$ contour interval 


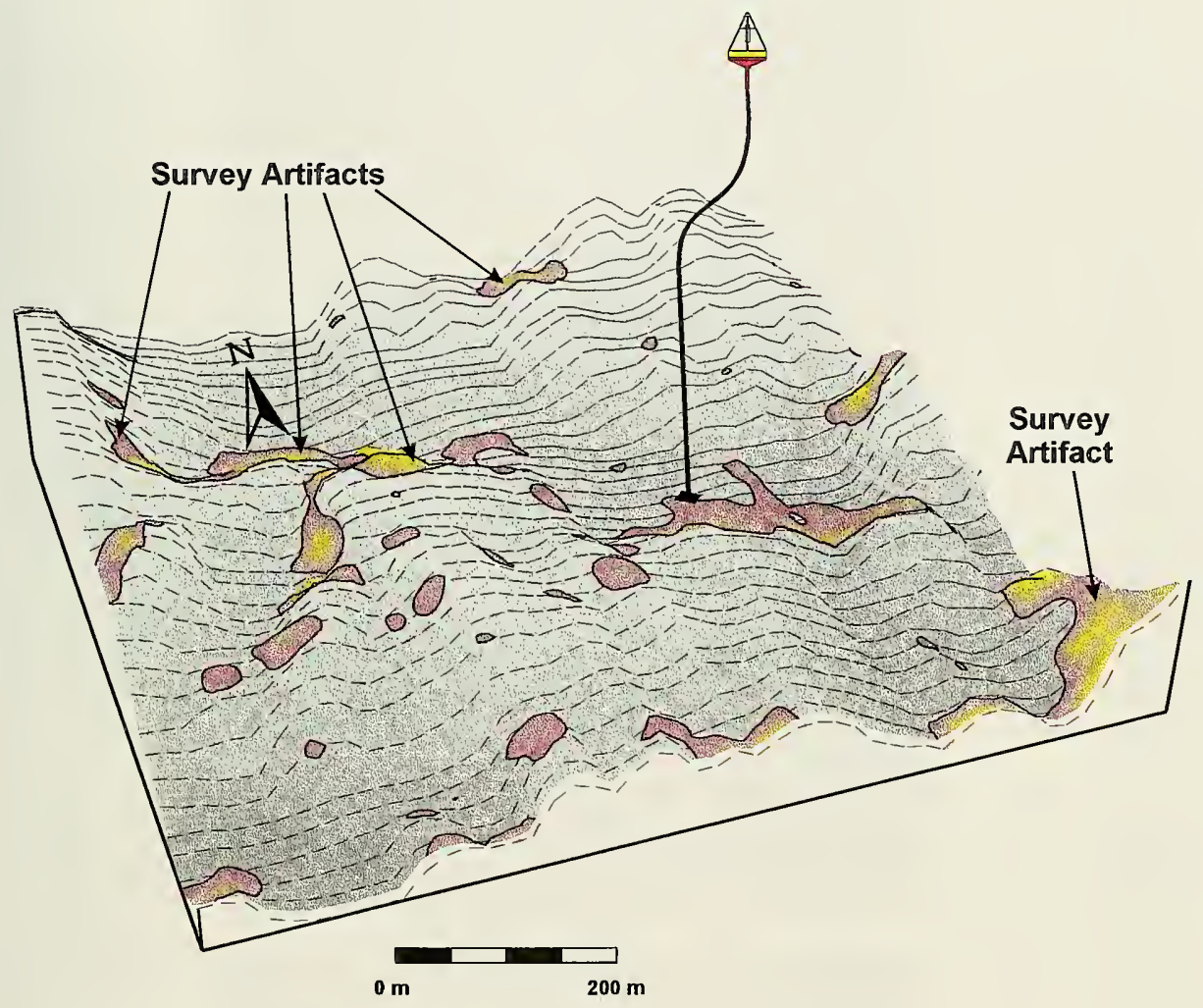

Figure 4-13. Three-dimensional view of the $800 \mathrm{~m} \times 800 \mathrm{~m}$ survey area over Portland Disposal Site, showing patterns of accumulation and survey artifacts with respect to bathymetric features 


\subsubsection{REMOTS ${ }^{\circledR}$ Sediment-Profile Photography}

A total of 33 REMOTS $^{\circledR}$ camera stations were occupied as part of the November 1996 field operations. The sediment-profile images collected in close proximity to the pseudo-UDM deposit were used to characterize the Royal River material as dark silt intermixed (mottled) with a light gray, stiff clay (representative of the glaciomarine deposit; Figure 4-14A). Plant material and wood fragments were visible in several of the replicate photographs obtained within the project area. Clumps of dredged material detected on the surface of the seafloor, and chaotic sediment fabric at the sediment/water interface, suggested that cohesive masses of sediments were released from the barges, descended more than $60 \mathrm{~m}$ through the water column, and were deposited intact (Figure 4$14 \mathrm{~B}$ and $\mathrm{C})$.

The thickness of fresh dredged material was mapped using the REMOTS ${ }^{\circledR}$ camera penetration data (Table 4-3). At many stations within $200 \mathrm{~m}$ of the center, the thickness of pseudo-UDM exceeded camera penetration; penetration depths ranged from 9-17 cm (Figure 4-15). The $>10 \mathrm{~cm}$ dredged material thickness contour incorporated all stations within $100 \mathrm{~m}$ of the survey center, as well as at Stations 125ESE, 200ENE, and 200S (Figures 4-15 and 4-16A). A few replicates at 50S, 50N, and $100 \mathrm{~S}$ indicated 3.5 to 8.8 $\mathrm{cm}$ of pseudo-UDM over ambient. Station 200SE had no penetration, consistent with the baseline survey (Figure 4-7).

Recently deposited Royal River material over ambient sediments, or historic dredged material in the far northwestern stations, was detected at many of the peripheral stations. A fine apron of material was measured at a few stations surrounding the main pseudo-UDM footprint, with thin layers $(<4 \mathrm{~cm})$ of material measured at $200 \mathrm{~W}, 300 \mathrm{~S}$, and 300SE. With the exception of Stations 200N, 400SE, 400S, and 400NW, layers of recently deposited dredged material with thicknesses $>1 \mathrm{~cm}$ covered the majority of the survey grid (Figure 4-16B).

The Royal River dredged material deposit was quickly recolonized by both small and large benthic fauna, verifying the suitability of the project pseudo-UDM for unconfined open water disposal (Figure 4-17). At one week postdisposal, RPD depths ranging from $1 \mathrm{~cm}$ to $4 \mathrm{~cm}$ were detected in the majority of the sediment-profile images.

\subsubsection{Sediment Grab Sampling}

At nine of the REMOTS ${ }^{\circledR}$ stations, surface sediment grab samples were collected over the pseudo-UDM deposit formed near the center of the Royal River Project Area. The sediment recovered was sampled, preserved, and analyzed for grain size and the fine fraction composition. 

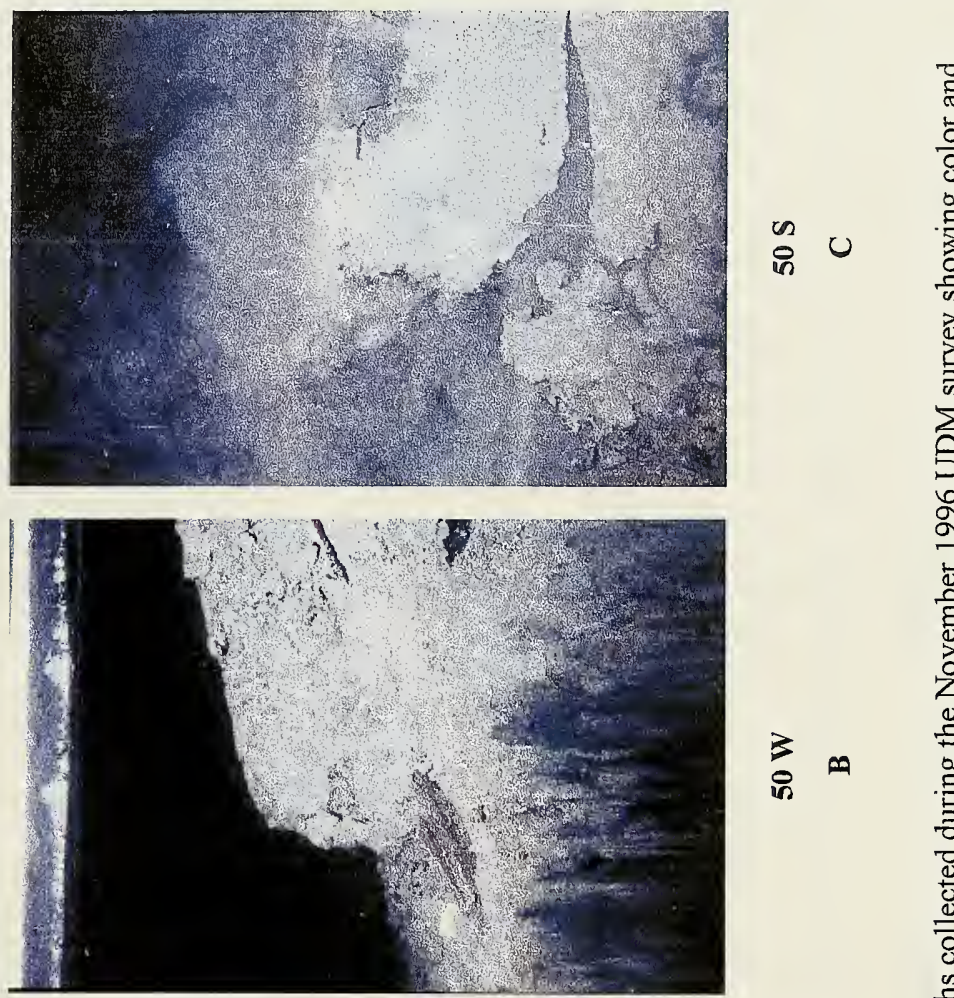

명

8

ก $U$

ำ
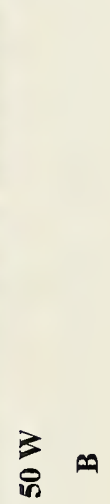

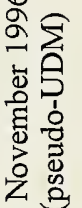

d

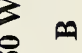

品惫

完要

苍 总

兽

8 เ

参, 空
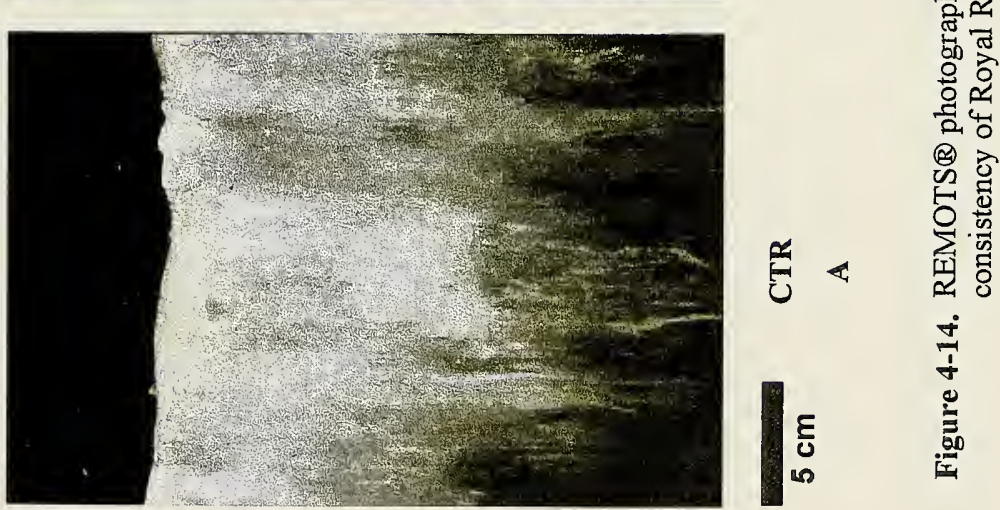


\section{Portland Disposal Site Royal River Project Area Precap REMOTS $₫$ Survey Stations over Precap Bathymetry}

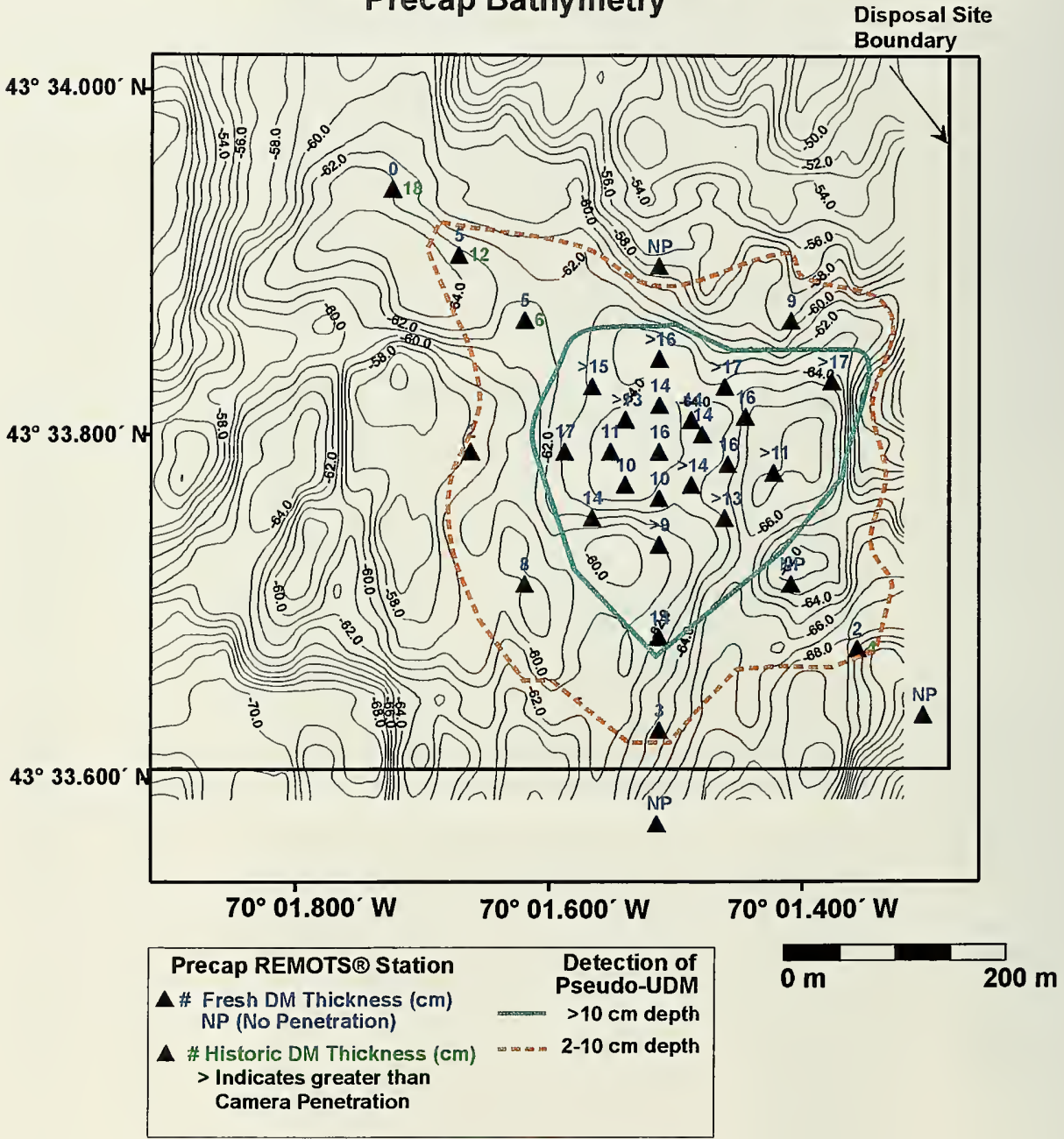

Figure 4-15. Thickness of pseudo-UDM measured by REMOTS $\otimes$ data overlaid on precap bathymetric chart (NAD 27), $1.0 \mathrm{~m}$ contour interval (MLLW)

The Portland Disposal Site Capping Demonstration Project, 1995-1997 


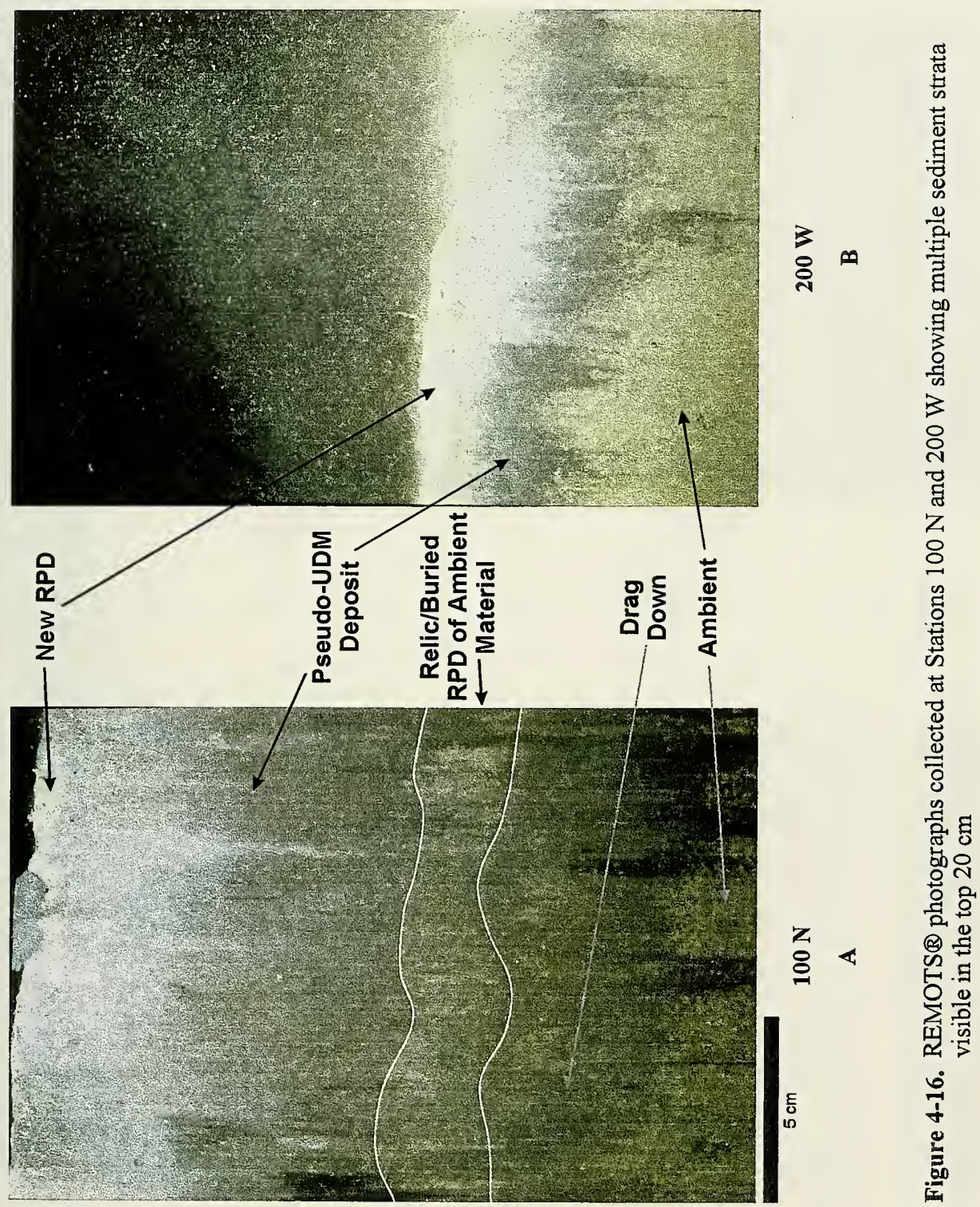



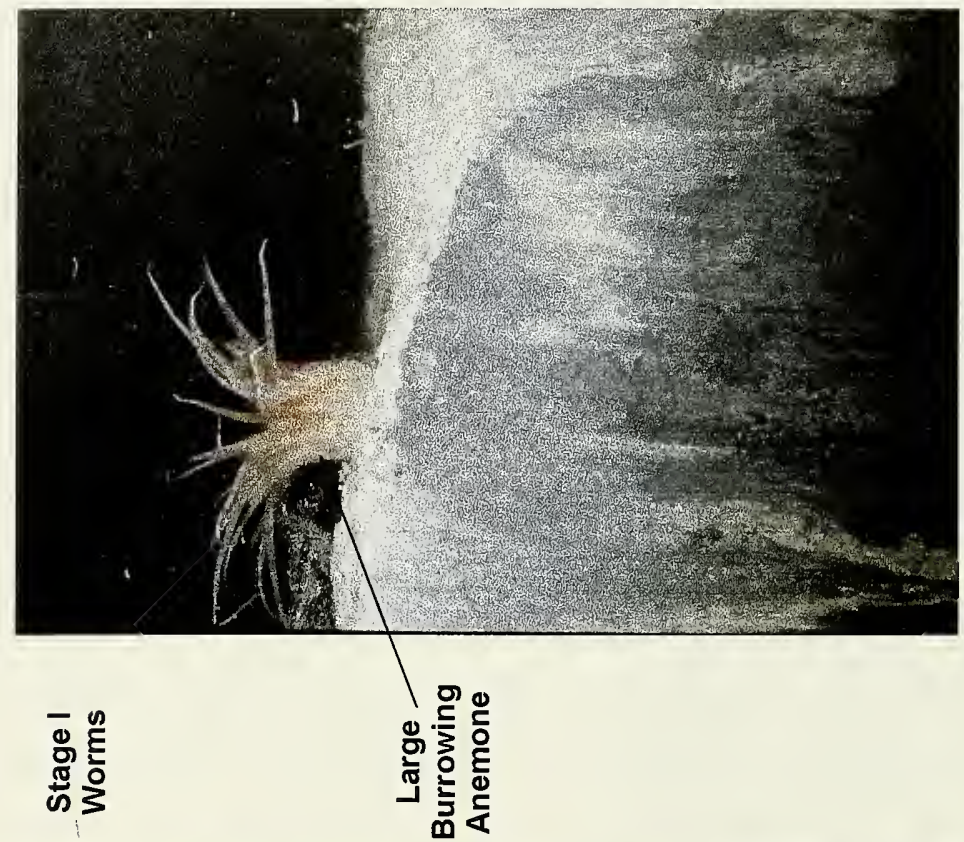

Z

告

菂

응

동

莺

ज

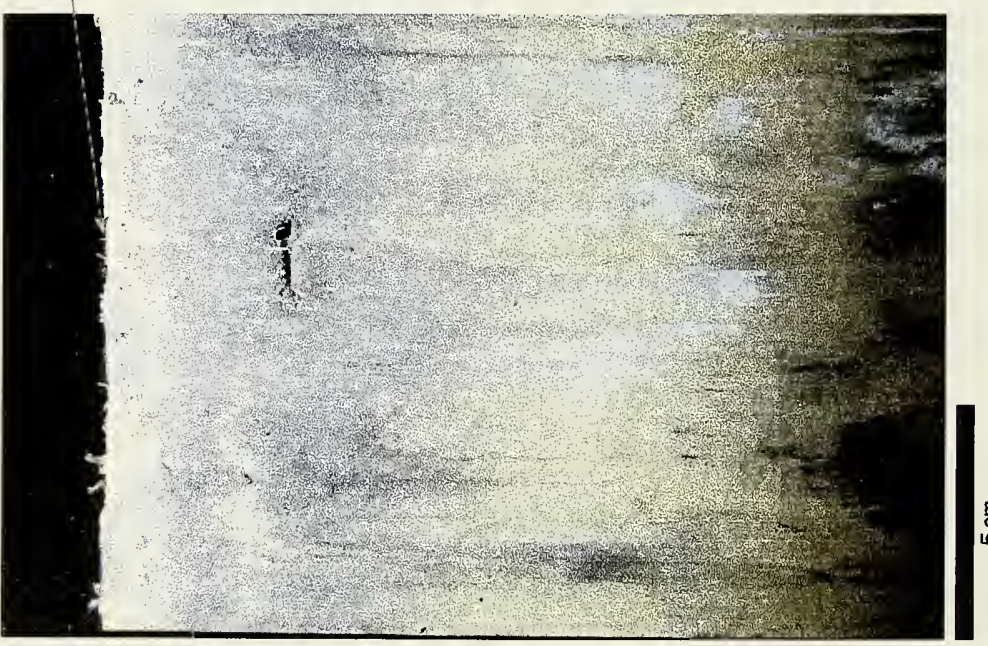

प्ठ

¿

응

ज्दे

ते

th

융

E

을

四

영 울

น

응 킁

$\sum_{0}$

도용

굴 


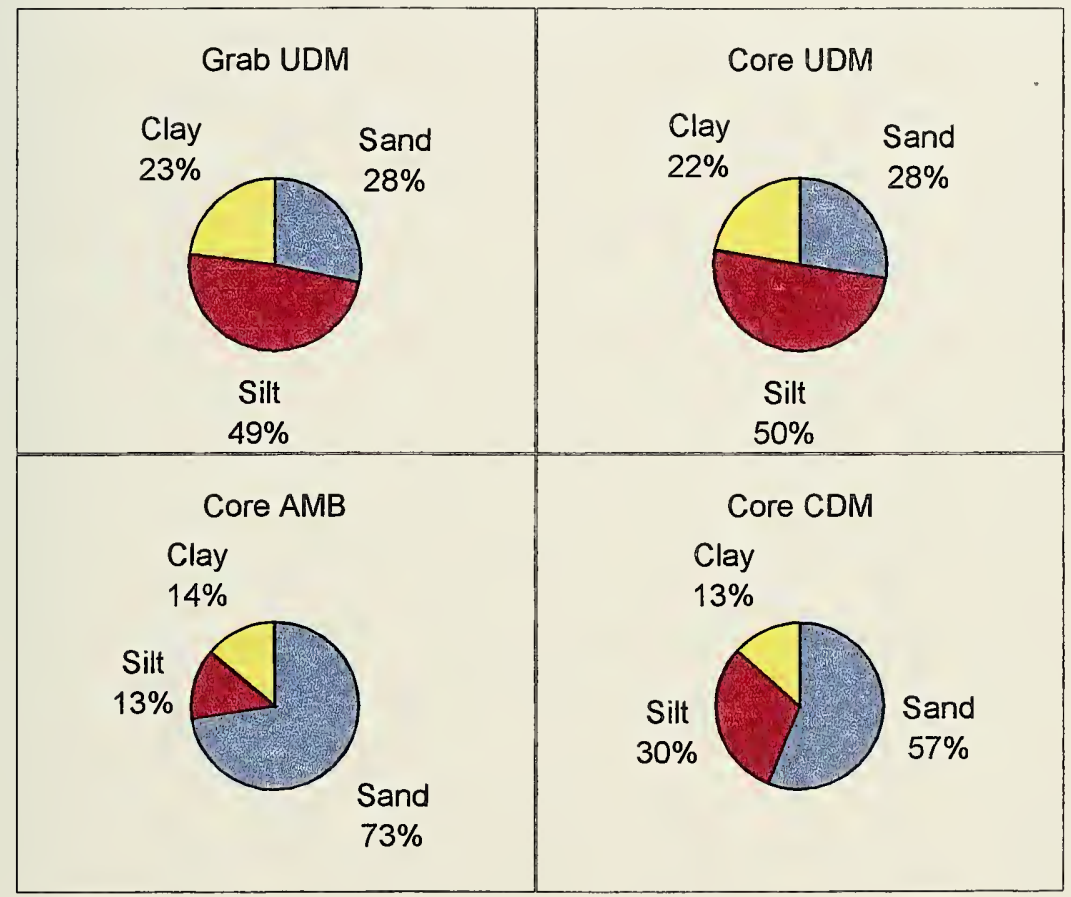

Figure 4-18. Mean grain size analysis results for precap (pseudo-UDM) grab samples and postcap (CDM) core samples. Core layers determined by visual observations and microfossil content 
Table 4-3

November 1996 Precap REMOTS ${ }^{\circledR}$ Sediment-Profile Photography Survey Results Showing Detection of Fresh Pseudo-UDM and Historic Dredged Material

\begin{tabular}{|c|c|c|c|c|c|c|c|c|c|c|c|}
\hline \multirow{2}{*}{$\begin{array}{c}\text { Precap Survey } \\
\text { Station }\end{array}$} & \multirow[t]{2}{*}{ Replicate } & \multicolumn{3}{|c|}{ Camera Penetration (cm) } & \multirow{2}{*}{$\begin{array}{c}\text { Station } \\
\text { Average }\end{array}$} & \multicolumn{3}{|c|}{ Pseudo-UDM Thickness } & \multicolumn{3}{|c|}{ Historic DM Thickness } \\
\hline & & Min & Max & Average & & $(\mathrm{cm})$ & >pen* & Average & $(\mathrm{cm})$ & $>$ pen* & Average \\
\hline CTR & $\bar{A}$ & 15 & 15.5 & 15.25 & 16.09 & 14.8 & & 15.7 & 0 & & 0.0 \\
\hline CTR & $\mathbf{B}$ & 17 & 17.5 & 17.25 & & 16.8 & & & 0 & & \\
\hline CTR & $\mathrm{C}$ & 15 & 15.75 & 15.38 & & 14.8 & & & 0 & & \\
\hline CTR & $\mathrm{D}$ & 16 & 17 & 16.50 & & 16.5 & $>$ pen & & 0 & & \\
\hline $50 \mathrm{~N}$ & $\mathbf{A}$ & 18.75 & 19.25 & 19.00 & 14.69 & 18 & & 13.7 & 0 & & 0.0 \\
\hline $50 \mathrm{~N}$ & $\mathrm{~B}$ & 18.5 & 18.75 & 18.63 & & 17 & & & 0 & & \\
\hline $50 \mathrm{~N}$ & $\mathrm{C}$ & 10 & 10.25 & 10.13 & & 8.8 & & & 0 & & \\
\hline $50 \mathrm{~N}$ & D & 8.25 & 13.75 & 11.00 & & 11 & $>$ pen & & 0 & & \\
\hline $100 \mathrm{~N}$ & $\mathrm{~A}$ & OVER PEN & & $\mathrm{NA}$ & 16.03 & $\mathrm{NA}$ & & 15.8 & $\mathrm{NA}$ & & 0.0 \\
\hline $100 \mathrm{~N}$ & B & OVER PEN & & $\mathrm{NA}$ & & NA & & & NA & & \\
\hline $100 \mathrm{~N}$ & $\mathrm{C}$ & OVER PEN & & $\mathrm{NA}$ & & $\mathrm{NA}$ & & & $\mathrm{NA}$ & & \\
\hline $100 \mathrm{~N}$ & D & 16 & 16.75 & 16.38 & & 15.5 & & & 0 & & \\
\hline $100 \mathrm{~N}$ & $E$ & 10.25 & 12.75 & 11.50 & & 11.5 & $>$ pen & & 0 & & \\
\hline $100 \mathrm{~N}$ & $\mathbf{F}$ & 16.5 & 17 & 16.75 & & 16.8 & $>$ pen & & 0 & & \\
\hline $100 \mathrm{~N}$ & G & 19 & 20 & 19.50 & & 19.5 & $>$ pen & & 0 & & \\
\hline $200 \mathrm{~N}$ & $\mathrm{~A}$ & NOPEN & & $\mathrm{NA}$ & $\mathrm{NA}$ & NA & & NA & $\mathrm{NA}$ & & NA \\
\hline $200 \mathrm{~N}$ & B & NO PEN & & NA & & NA & & & NA & & \\
\hline $200 \mathrm{~N}$ & C & NO PEN & & $\mathrm{NA}$ & & NA & & & NA & & \\
\hline $200 \mathrm{~N}$ & D & NO PEN & & NA & & $\mathrm{NA}$ & & & $\mathrm{NA}$ & & \\
\hline 50NE & $\mathbf{A}$ & 17.25 & 19.75 & 18.50 & 14.13 & 19.25 & $>$ pen & 14.2 & 0 & & 0.0 \\
\hline $50 \mathrm{NE}$ & B & 12.5 & 13.75 & 13.13 & & 12.5 & & & 0 & & \\
\hline 50NE & C & 8.5 & 9.75 & 9.13 & & 9.1 & $>$ pen & & 0 & & \\
\hline 50NE & D & 13.5 & 18 & 15.75 & & 15.8 & $>$ pen & & 0 & & \\
\hline $100 \mathrm{NE}$ & $\mathrm{A}$ & 15.25 & 17.5 & 16.38 & 17.38 & 16.4 & $\geq$ pen & 17.4 & 0 & & 0.0 \\
\hline $100 \mathrm{NE}$ & B & OVER PEN & & $\mathrm{NA}$ & & NA & & & NA & & \\
\hline $100 \mathrm{NE}$ & C & 17.75 & 19 & 18.38 & & 18.4 & $>$ pen & & 0 & & \\
\hline 200NE & A & NO PEN & & NA & 10.38 & $\mathrm{NA}$ & & 9.2 & NA & & 0.0 \\
\hline 200NE & B & NO PEN & & $\mathrm{NA}$ & & NA & & & NA & & \\
\hline $200 \mathrm{NE}$ & C & 5.5 & 7 & 6.25 & & 6.3 & $>$ pen & & 0 & & \\
\hline 200NE & D & NO PEN & & $\mathrm{NA}$ & & $\mathrm{NA}$ & & & $\mathrm{NA}$ & & \\
\hline $200 \mathrm{NE}$ & E & NO PEN & & $\mathrm{NA}$ & & $\mathrm{NA}$ & & & $\mathrm{NA}$ & & \\
\hline 200NE & $\mathbf{F}$ & 9 & 10.5 & 9.75 & & 9.8 & & & 0 & & \\
\hline 200NE & G & 15 & 15.25 & 15.13 & & 11.5 & & & 0 & & \\
\hline 50ENE & A & 13.5 & 14.25 & 13.88 & 14.16 & 13 & & 13.8 & 0 & & 0.0 \\
\hline 50ENE & B & 10.75 & 11.75 & 11.25 & & 11.3 & $>$ pen & & 0 & & \\
\hline 50ENE & $\mathrm{C}$ & 15.75 & 16.75 & 16.25 & & 16.3 & $>$ pen & & 0 & & \\
\hline 50ENE & $\mathbf{D}$ & 14.75 & 15.75 & 15.25 & & 14.5 & & & 0 & & \\
\hline 100ENE & $\mathrm{A}$ & 17.25 & 19 & 18.13 & 16.67 & 17 & & 15.5 & 0 & & 0.0 \\
\hline 100ENE & B & 16 & 18 & 17.00 & & 15 & & & 0 & & \\
\hline 100ENE & C & 14.25 & 15.5 & 14.88 & & 14.5 & & & 0 & & \\
\hline 200ENE & $\mathrm{A}$ & OVER PEN & & NA & 17.31 & $\mathrm{NA}$ & & 17.2 & $\mathrm{NA}$ & & 0.0 \\
\hline 200ENE & B & OVER PEN & & $\mathrm{NA}$ & & $\mathrm{NA}$ & & & $\mathrm{NA}$ & & \\
\hline 200ENE & $\mathrm{C}$ & 16.25 & 18 & 17.13 & & 17.1 & $>$ pen & & 0 & & \\
\hline 200ENE & D & 15 & 15.25 & 15.13 & & 14.8 & >pen & & 0 & & \\
\hline 200ENE & $E$ & 18 & 19 & 18.50 & & 18.5 & $>$ pen & & 0 & & \\
\hline 200ENE & $\mathbf{F}$ & 17 & 20 & 18.50 & & 18.5 & $>$ pen & & 0 & & \\
\hline
\end{tabular}

* ">pen" indicates thickness greater than the camera penetration; where not specified, ambient sediments were below the measured dredged material. 
Table 4-3 (continued)

\begin{tabular}{|c|c|c|c|c|c|c|c|c|c|c|c|}
\hline \multirow{2}{*}{$\begin{array}{c}\text { Precap Survey } \\
\text { Station }\end{array}$} & \multirow[t]{2}{*}{ Replicate } & \multicolumn{3}{|c|}{ Camera Penetration (cm) } & \multirow{2}{*}{$\begin{array}{c}\text { Station } \\
\text { Average } \\
\end{array}$} & \multicolumn{3}{|c|}{ Pseudo-UDM Thickness } & \multicolumn{3}{|c|}{ Historic DM Thickness } \\
\hline & & Min & Max & Average & & $(\mathrm{cm})$ & >pen* & Average & $(\mathrm{cm})$ & $>$ pen* & Average \\
\hline 75ESE & $\mathrm{A}$ & 16.75 & 17.25 & 17.00 & 16.83 & 16.5 & & 16.0 & 0 & & 0.0 \\
\hline 75ESE & $\mathbf{B}$ & 18.75 & 19.5 & 19.13 & & 18 & & & 0 & & \\
\hline 75ESE & $\mathrm{C}$ & 14.25 & 14.5 & 14.38 & & 13.5 & & & 0 & & \\
\hline 125ESE & $\mathbf{A}$ & 7.75 & 16.5 & 12.13 & 11.09 & 12.1 & >pen & 11.1 & 0 & & 0.0 \\
\hline 125ESE & B & 11.5 & 12.5 & 12.00 & & 12 & $>$ pen & & 0 & & \\
\hline 125ESE & $\mathbf{C}$ & 16 & 16.75 & 16.38 & & 16.4 & $>$ pen & & 0 & & \\
\hline 125ESE & $\mathbf{D}$ & 2 & 5.75 & 3.88 & & 3.9 & >pen & & 0 & & \\
\hline S0SE & $\mathbf{A}$ & 12.75 & 13.5 & 13.13 & 14.09 & 13 & $>$ pen & 14.0 & 0 & & 0.0 \\
\hline 50SE & B & 14.5 & 15.25 & 14.88 & & 14.5 & $>$ pen & & 0 & & \\
\hline SOSE & $\mathrm{C}$ & 14 & 14.25 & 14.13 & & 14.1 & $>$ pen & & 0 & & \\
\hline 50SE & D & 13.75 & 14.75 & 14.25 & & 14.3 & >pen & & 0 & & \\
\hline 100SE & A & 9 & 12.5 & 10.75 & 13.30 & 10.8 & $>$ pen & 13.3 & 0 & & 0.0 \\
\hline 100SE & D & 14.75 & 15.5 & 15.13 & & 15 & >pen & & 0 & & \\
\hline 100SE & $\mathbf{E}$ & 15.25 & 17 & 16.13 & & 16.1 & $>$ pen & & 0 & & \\
\hline 100SE & $\mathbf{F}$ & 13.5 & 14.25 & 13.88 & & 13.9 & >pen & & 0 & & \\
\hline 100SE & G & 9 & 12.25 & 10.63 & & 10.6 & >pen & & 0 & & \\
\hline 200SE & A & NOPEN & & $\mathrm{NA}$ & NA & NA & & NA & $\mathrm{NA}$ & & NA \\
\hline 200SE & B & NOPEN & & NA & & $\mathrm{NA}$ & & & NA & & \\
\hline $200 \mathrm{SE}$ & C & NO PEN & & $\mathrm{NA}$ & & NA & & & $\mathrm{NA}$ & & \\
\hline 300SE & A & 2 & 3 & 2.50 & 13.16 & NA & & 2.3 & NA & & 4 \\
\hline 300SE & B & 17.75 & 17.75 & 17.75 & & 0 & & & 4 & & \\
\hline 300SE & $\mathrm{C}$ & 16.75 & 17 & 16.88 & & 2.5 & & & NA & & \\
\hline $300 \mathrm{SE}$ & $\mathbf{D}$ & 15 & 16 & 15.50 & & 4.5 & & & $\mathrm{NA}$ & & \\
\hline $400 \mathrm{SE}$ & $\mathbf{A}$ & NO PEN & & $\mathrm{NA}$ & $\mathrm{NA}$ & NA & & $\mathrm{NA}$ & NA & & NA \\
\hline $400 \mathrm{SE}$ & $\mathbf{B}$ & NO PEN & & $\mathrm{NA}$ & & $\mathrm{NA}$ & & & $\mathrm{NA}$ & & \\
\hline 400SE & C & NO PEN & & NA & & NA & & & $\mathrm{NA}$ & & \\
\hline $50 \mathrm{~S}$ & A & 16 & 18.5 & 17.25 & 10.94 & 18 & $>$ pen & 9.5 & 0 & & 0.0 \\
\hline $50 \mathrm{~S}$ & B & 6.5 & 8.25 & 7.38 & & 5 & & & NA & & \\
\hline $50 \mathrm{~S}$ & C & 3 & 11.25 & 7.13 & & 3.5 & & & 0 & & \\
\hline $50 \mathrm{~S}$ & D & 11.25 & 12.75 & 12.00 & & 11.5 & & & 0 & & \\
\hline $100 \mathrm{~S}$ & $\mathrm{~A}$ & 8.25 & 9 & 8.63 & 8.88 & 8.6 & >pen & 8.7 & 0 & & 0.0 \\
\hline $100 \mathrm{~S}$ & B & 4.5 & 5 & 4.75 & & 3.5 & & & 0 & & \\
\hline $100 \mathrm{~S}$ & $\mathrm{C}$ & 3.5 & 4 & 3.75 & & 3.5 & & & 0 & & \\
\hline $100 S$ & D & 4.75 & 6.75 & 5.75 & & 6.25 & $>$ pen & & 0 & & \\
\hline $100 \mathrm{~S}$ & $\mathbf{E}$ & 13.25 & 14 & 13.63 & & 13.6 & >pen & & 0 & & \\
\hline $100 \mathrm{~S}$ & $\mathbf{F}$ & 16 & 17.5 & 16.75 & & 16.8 & $>$ pen & & 0 & & \\
\hline $200 S$ & $\mathbf{A}$ & NO PEN & & $\mathrm{NA}$ & 13.56 & $\mathrm{NA}$ & & 13.5 & NA & & 0.0 \\
\hline $200 \mathrm{~S}$ & $\mathbf{B}$ & NO PEN & & $\mathrm{NA}$ & & NA & & & $\mathrm{NA}$ & & \\
\hline $200 S$ & C & NO PEN & & NA & & NA & & & $\mathrm{NA}$ & & \\
\hline $200 S$ & $\mathbf{D}$ & 14.5 & 15.25 & 14.88 & & 14.5 & $>$ pen & & 0 & & \\
\hline $200 \mathrm{~S}$ & $\mathbf{E}$ & 11.25 & 13.25 & 12.25 & & 12.5 & $>$ pen & & 0 & & \\
\hline $200 \mathrm{~S}$ & $\mathbf{F}$ & NOPEN & & $\mathrm{NA}$ & & NA & & & NA & & \\
\hline $200 S$ & $\mathbf{G}$ & NOPEN & & $\mathrm{NA}$ & & $\mathrm{NA}$ & & & $\mathrm{NA}$ & & \\
\hline $300 \mathrm{~S}$ & A & 12.5 & 12.75 & 12.63 & 9.71 & 3 & & 3.0 & 0 & & 0.0 \\
\hline $300 \mathrm{~S}$ & B & 9 & 9.25 & 9.13 & & 2.5 & & & 0 & & \\
\hline $300 \mathrm{~S}$ & C & NOPEN & & $\mathrm{NA}$ & & NA & & & $\mathrm{NA}$ & & \\
\hline
\end{tabular}

* ">pen" indicates thickness greater than the camera penetration; where not specified, ambient sediments were below the measured dredged material. 
Table 4-3 (continued)

\begin{tabular}{|c|c|c|c|c|c|c|c|c|c|c|c|}
\hline \multirow{2}{*}{$\begin{array}{c}\text { Precap Survey } \\
\text { Station } \\
\end{array}$} & \multirow[t]{2}{*}{ Replicate } & \multicolumn{3}{|c|}{ Camera Penetration (cm) } & \multirow{2}{*}{\begin{tabular}{c|} 
Station \\
Average
\end{tabular}} & \multicolumn{3}{|c|}{ Pseudo-UDM Thickness } & \multicolumn{3}{|c|}{ Historic DM Thickness } \\
\hline & & Min & Max & Average & & $(\mathrm{cm})$ & $>p_{\text {pen }}^{*}$ & Average & $(\mathrm{cm})$ & $>$ pen* & Average \\
\hline $300 \mathrm{~S}$ & D & 7 & 7.75 & 7.38 & & 3.5 & & & 0 & & \\
\hline $400 \mathrm{~S}$ & $\mathbf{A}$ & NOPEN & & $\mathrm{NA}$ & NA & $\mathrm{NA}$ & & NiA & $\mathrm{NA}$ & & NA \\
\hline $400 \mathrm{~S}$ & $\mathbf{B}$ & NOPEN & & NA & & NA & & & NA & & \\
\hline $50 \mathrm{SW}$ & $\mathrm{A}$ & 9.25 & 12.25 & 10.75 & 9.54 & 11.5 & & 9.7 & 0 & & 0.0 \\
\hline $50 \mathrm{SW}$ & B & 13.5 & 13.75 & 13.63 & & 13.5 & >pen & & 0 & & \\
\hline $50 \mathrm{SW}$ & $\mathrm{C}$ & 3 & 5.5 & 4.25 & & 4 & & & 0 & & \\
\hline $50 \mathrm{SW}$ & $\mathbf{D}$ & NOPEN & & $\mathrm{NA}$ & & NA & & & $\mathrm{NA}$ & & \\
\hline $100 \mathrm{SW}$ & A & NO PEN & & NA & 17.38 & $\mathrm{NA}$ & & -14.1 & $\mathrm{NA}$ & & 0.0 \\
\hline $100 \mathrm{SW}$ & B & 19 & 19.75 & 19.38 & & 12 & & & 0 & & \\
\hline $100 \mathrm{SW}$ & $\mathrm{C}$ & 18.75 & 19 & 18.88 & & 13 & & & 0 & & \\
\hline $100 S W$ & D & 14 & 15.25 & 14.63 & & 14.8 & $>$ pen & & 0 & & \\
\hline $100 \mathrm{SW}$ & $\mathbf{E}$ & 15.25 & 18 & 16.63 & & 16.6 & $>$ pen & & 0 & & \\
\hline $200 \mathrm{SW}$ & $\mathbf{A}$ & 0.75 & 2 & 1.38 & 9.95 & $\mathrm{NA}$ & & 8.0 & $\mathrm{NA}$ & & 0.0 \\
\hline $200 \mathrm{SW}$ & $\mathbf{B}$ & NO PEN & & $\mathrm{NA}$ & & NA & & & NA & & \\
\hline $200 \mathrm{SW}$ & D & 14.25 & 35 & 14.63 & & 10.5 & & & 0 & & \\
\hline $200 \mathrm{SW}$ & $E$ & 17.75 & 18.5 & 18.13 & & 8 & & & 0 & & \\
\hline $200 S w$ & $\mathrm{~F}$ & 4.75 & 7.75 & 6.25 & & 4 & & & 0 & & \\
\hline 200 SW & $\mathbf{G}$ & 9 & 9.75 & 9.38 & & 9.4 & >pen & & 0 & & \\
\hline $50 \mathrm{~W}$ & $\mathbf{A}$ & 6 & 8.5 & 7.25 & 12.13 & 5.8 & & 11.5 & 0 & & 0.0 \\
\hline $50 \mathrm{~W}$ & $\mathbf{B}$ & 18.25 & 18.5 & 18.38 & & 17.5 & & & 0 & & \\
\hline $50 \mathrm{~W}$ & C & 10 & 11.75 & 10.88 & & 10.5 & $>$ pen & & 0 & & \\
\hline $50 \mathrm{~W}$ & $\mathrm{D}$ & 11.5 & 12.5 & 12.00 & & 12 & $>$ pen & & 0 & & \\
\hline $100 \mathrm{~W}$ & $\mathbf{A}$ & OVER PEN & & $\mathrm{NA}$ & 17.41 & $\mathrm{NA}$ & & 17.3 & $\mathrm{NA}$ & & 0.0 \\
\hline $100 \mathrm{~W}$ & B & 15.25 & 18.25 & 16.75 & & 16.8 & $>$ pen & & 0 & & \\
\hline $100 \mathrm{~W}$ & C & 18.5 & 18.75 & 18.63 & & 18.6 & $>$ pen & & 0 & & \\
\hline $100 \mathrm{~W}$ & D & 16.25 & 16.75 & 16.50 & & 16.3 & $>$ pen & & 0 & & \\
\hline $100 \mathrm{~W}$ & $\mathbf{E}$ & 17.5 & 18 & 17.75 & & 17.3 & $>$ pen & & 0 & & \\
\hline $200 \mathrm{~W}$ & A & 8 & 8.5 & 8.25 & 11.42 & 2 & & 2.0 & 0 & & 0.0 \\
\hline $200 \mathrm{~W}$ & $\mathbf{B}$ & 15.5 & 16 & 15.75 & & 1 & & & 0 & & \\
\hline $200 W$ & D & NO PEN & & $\mathrm{NA}$ & & NA & & & $\mathrm{NA}$ & & \\
\hline $200 \mathrm{~W}$ & $\mathbf{E}$ & 10 & 10.5 & 10.25 & & 3 & & & 0 & & \\
\hline $50 \mathrm{NW}$ & A & 9 & 12 & 10.50 & 12.63 & 10.5 & $>$ pen & 12.6 & 0 & & 0.0 \\
\hline $50 \mathrm{NW}$ & $\mathbf{B}$ & 10 & 11.25 & 10.63 & & 10.8 & $>$ pen & & 0 & & \\
\hline $50 N W$ & $\mathrm{C}$ & 16.5 & 17 & 16.75 & & 16.5 & $>$ pen & & 0 & & \\
\hline $100 \mathrm{NW}$ & A & 18.5 & 19 & 18.75 & 16.96 & 11 & & 14.5 & 0 & & 0.0 \\
\hline $100 \mathrm{NW}$ & B & 12.5 & 16.75 & 14.63 & & 15 & $>$ pen & & 0 & & \\
\hline $100 \mathrm{NW}$ & $\mathrm{C}$ & 17 & 18 & 17.50 & & 17.5 & $>$ pen & & 0 & & \\
\hline $200 \mathrm{NW}$ & $\mathbf{A}$ & 14.5 & 14.75 & 14.63 & 12.88 & 5.5 & & 5.3 & 7.5 & & 6.0 \\
\hline $200 \mathrm{NW}$ & B & 11 & 11.25 & 11.13 & & 5 & & & 4.5 & & \\
\hline $300 \mathrm{NW}$ & $\mathbf{A}$ & 15 & 18 & 16.50 & 17.13 & 6.5 & & 5.0 & 10 & & 12.2 \\
\hline $300 \mathrm{NW}$ & B & 15.25 & 16 & 15.63 & & 5 & & & 10.6 & & \\
\hline $300 \mathrm{NW}$ & $\mathrm{C}$ & 19 & 19.5 & 19.25 & & 3.5 & & & 16 & $>$ pen & \\
\hline $400 \mathrm{NW}$ & $\mathbf{A}$ & 17.5 & 18.5 & 18.00 & 17.71 & 0 & & 0.0 & 18 & >pen & 17.7 \\
\hline $400 \mathrm{NW}$ & B & 18.25 & 19.5 & 18.88 & & 0 & & & 18.9 & $>$ pen & \\
\hline $400 \mathrm{NW}$ & $\mathrm{C}$ & 15.5 & 17 & 16.25 & & 0 & & & 16.3 & $>$ pen & \\
\hline
\end{tabular}

* ">pen" indicates thickness greater than the camera penetration; where not specified, ambient sediments were below the measured dredged material. 
Table 4-4

Precap (UDM) Grab Sample Survey Grain Size Analysis Results

\begin{tabular}{|l|l|c|c|c|}
\hline Survey & Station & \% Sand & \% Silt & \% Clay \\
\hline UDM Grab & $50 \mathrm{~N}$ & 38.5 & 40.9 & 20.6 \\
\hline UDM Grab & $50 \mathrm{~S}$ & 17.1 & 56.9 & 26.1 \\
\hline UDM Grab & $50 \mathrm{~W}$ & 30.9 & 47.1 & 22.0 \\
\hline UDM Grab & $50 \mathrm{ENE}$ & 36.9 & 42.6 & 20.5 \\
\hline UDM Grab & $50 \mathrm{SE}$ & 10.7 & 63.8 & 25.5 \\
\hline UDM Grab & $50 \mathrm{NW}$ & 36.0 & 44.5 & 19.5 \\
\hline UDM Grab & CTR & 18.6 & 56.3 & 25.2 \\
\hline UDM Grab & $50 \mathrm{SW}$ & 34.4 & 40.2 & 25.4 \\
\hline UDM Grab & $50 \mathrm{NE}$ & 32.3 & 45.9 & 21.9 \\
\hline \hline UDM Grab & Average & 28.4 & 48.7 & 23.0 \\
\hline
\end{tabular}

Table 4-5

Precap (Pseudo-UDM) Grab Sample Fine Fraction Mineralogy Results

\begin{tabular}{|c|c|c|c|c|c|c|c|c|c|c|c|c|c|c|c|c|}
\hline 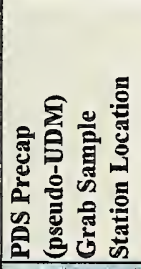 & 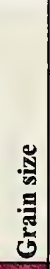 & 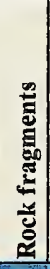 & 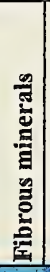 & 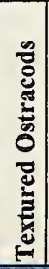 & 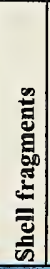 & $\begin{array}{l} \pm \\
5 \\
5 \\
0 \\
0\end{array}$ & 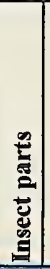 & 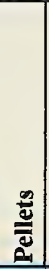 & 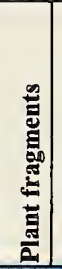 & 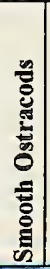 & 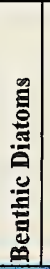 & 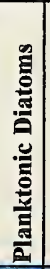 & 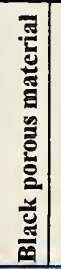 & 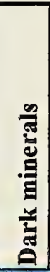 & 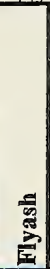 & Key \\
\hline $\begin{array}{l}\text { CTR } \\
50 \mathrm{~N}\end{array}$ & C & $\mathrm{X}$ & & $x$ & & & $\bar{x}$ & & $\mathrm{xx}$ & $\bar{x}$ & & $\bar{X}$ & $\frac{x}{x y}$ & $x$ & $\mathrm{x}$ & Min. Parameter \\
\hline $50 \mathrm{~S}$ & $\mathrm{M}$ & & & $\mathrm{X}$ & & & $X$ & $\mathrm{x}$ & $x x^{x}$ & & & $\mathrm{X}$ & $x \times$ & & & XX Abundant \\
\hline $50 \mathrm{~W}$ & C. & & & & & & $\mathrm{x}$ & $x$ & $\mathrm{x}$ & $\mathrm{x}$ & & $x$ & $\mathrm{x}$ & & $\mathrm{X}$ & X Common \\
\hline $50 \mathrm{NW}$ & $C$ & & & $\mathrm{x}$ & & & $\mathrm{x}$ & & $x$ & $X$ & & $\mathrm{x}$ & $\mathrm{x}$ & & $x$ & $x$ Rare \\
\hline 50ENE & & & & & & & $x_{2}$ & $\mathrm{x}$ & $\mathrm{x}$ & $\mathrm{x}$ & & $\mathrm{x}$ & $\mathrm{x}$ & & $\mathrm{x}$ & Absent \\
\hline $50 \mathrm{NE}-\mathrm{Me}$ & M & 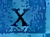 & & $\mathrm{X}$ & & & $x$ & $\mathrm{x}$ & 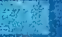 & $\mathrm{x}$ & & $\mathrm{x}$ & $\mathrm{X}$ & $\mathrm{x}$ & & Graim size \\
\hline $50 \mathrm{NE}-\mathrm{RBF}$ & $\mathrm{M}$ & & & & & & $\mathrm{x}$ & $\mathrm{X}$ & $\mathrm{x}$ & $\mathrm{x}$ & & $\mathrm{X}$ & $x$ & & & $\overline{C \text { Coarse }}$ \\
\hline $50 \mathrm{SW}$ & $\mathrm{M}$ & $\mathrm{x}$ & & $\mathrm{X}$ & $x$ & & $\mathrm{x}$ & $\mathrm{x}$ & $\mathrm{X}$ & $\mathrm{X}$ & & $x$ & $\mathrm{x}$ & & & M Medium \\
\hline $50 \mathrm{SE}$ & M. & & & $\mathrm{x}$ & & & $\mathrm{X}$ & $\mathrm{x}$ & $\mathrm{X}$ & $\mathrm{x}$ & & $\mathrm{x}$ & $\mathrm{X}$ & & & F Fine \\
\hline
\end{tabular}




\subsubsection{Grain Size Analysis}

Grain size analyses indicated that the average pseudo-UDM sample had a comparable grain size to the organic-rich material collected from the upper reaches of the Royal River. The mean grain size distribution for the sediment forming the pseudo-UDM mound was $25 \%$ sand, $44 \%$ silt, and $21 \%$ clay (Table $4-4$; Figure $4-18$ ). For most samples, the percent composition ranged from $31 \%$ to $38 \%$ for sand, and $40 \%$ to $47 \%$ for silt, except for samples CTR, 50S, and 50SE which had less sand (11-18\%) and more silt $(56-63 \%)$. The clay content within the mound sediments was consistent with percent composition ranging from $20-26 \%$.

\subsubsection{Fine Fraction}

Mineralogy. As in the August 1995 Royal River cores, mineralogical analysis (based on visual description) of the nine pseudo-UDM grab samples showed that quartz was dominant and micas common. Sand-sized particles retained in a $62.5 \mu \mathrm{m}$ sieve were classified as medium to coarse (2.0 to 1.0 phi; Table $4-5)$. Black porous material and plant fragments, in all but the methanol-preserved sample, were common to abundant (Figure 419). Insect parts, planktonic diatoms, and pellets were common in nearly all samples. Flyash, rock fragments, as well as textured and smooth ostracods were common in most samples. Fibrous minerals and shell fragments were very rare, and benthic diatoms were absent.

Microfossils. The pseudo-UDM grab samples contained all five groups of microfossils (thecamoebians and foraminifera: marsh, mudflat, shelf agglutinated and shelf calcareous); Figure 4-20A). Marsh and mudflat foraminifera were dominant in all 10 sediment samples analyzed, while freshwater thecamoebians composed from $5 \%$ to $30 \%$ of the individuals counted. With the exception of the sample collected at Station CTR and the methanol-preserved sample from 50NE, shelf calcareous species were collected from the surface of the pseudo-UDM deposit (percent abundance ranging from $2 \%$ to $20 \%$ ), suggesting rapid recolonization of the dredged material by shelf species. Two samples, 50SE and 50S, contained a few shelf agglutinated species and had a significantly greater density of foraminifera than the other samples (Figure 4-20B). The thecamoebians had a consistent density ranging from 4 to 20 individuals per gram. Foraminifera varied in density, with most samples ranging from 40 to 120 individuals per gram.

Both samples (methanol and formalin) from Station 50NE were processed and analyzed in order to compare microfossil content. Microfossil analyses determined the sample preserved in formalin solution contained nearly double the density of foraminifera relative to the sample preserved in methanol. After picking five trays of sediment, there 


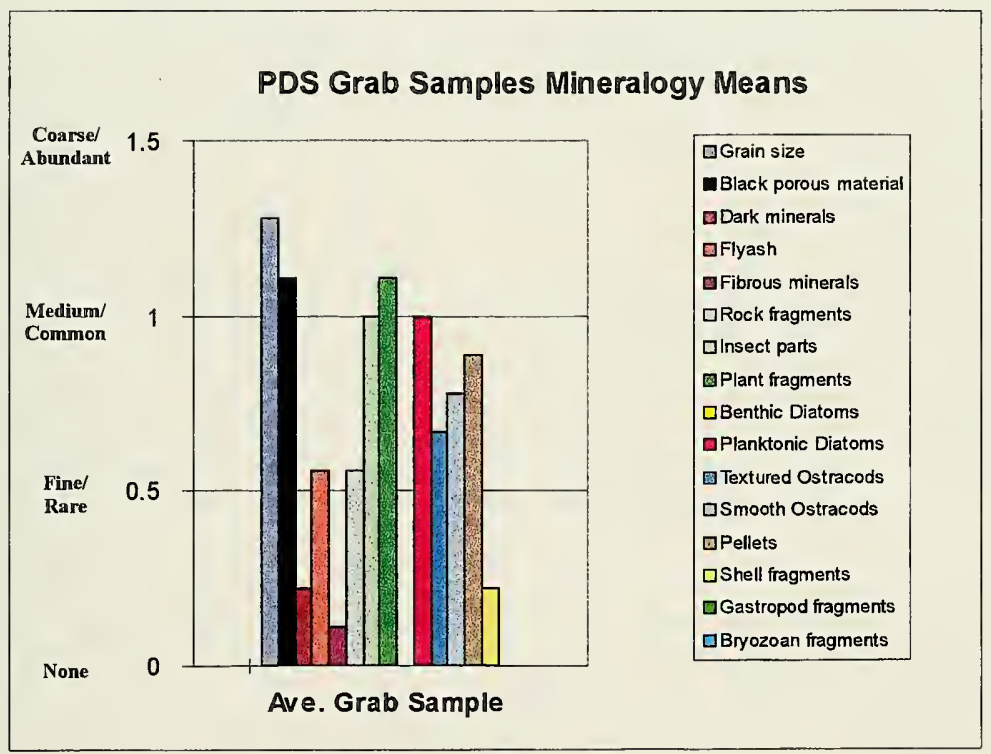

Figure 4-19. Histogram displaying mean abundance (rare, common, abundant) and particle size (fine, medium, coarse) for the mineralogical components within the fine fraction of grab samples collected during the precap (pseudo-UDM) survey 

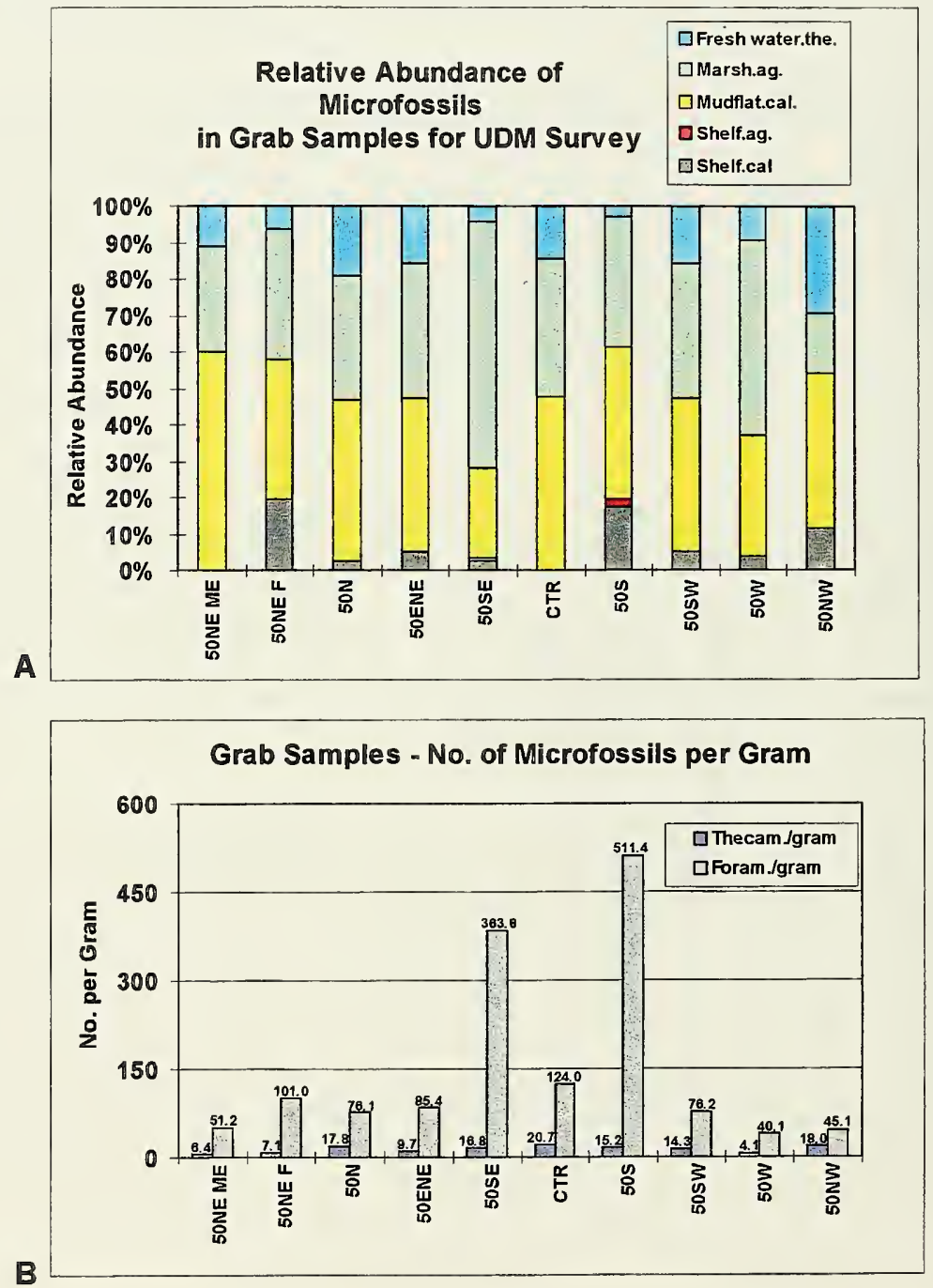

Figure 4-20. Histograms showing relative abundance and density of microfossils collected within the ten processed grab samples (methanol $[\mathrm{ME}]$ and formalin $[\mathrm{F}]$ at $50 \mathrm{NE}$ only) 
were 71 foraminifera (27 agglutinated, 44 calcareous) in the formalin sample and 40 (13 agglutinated, 27 calcareous) in the methanol sample. No shelf calcareous foraminifera were found in the methanol sample, while 15 Ammonia falsobeccarii were found in the formalin sample. Both the methanol and buffered formalin preserved samples contained five thecamoebians. As a result of this comparison, of the remaining 17 samples, only those preserved in formalin were processed and analyzed for microfossil content.

\subsection{Royal River Project Area Postcap Survey}

In mid-January 1997, SAIC conducted a postcap survey, including: precision bathymetry, REMOTS ${ }^{\circledast}$ sediment-profile photography, and sediment coring to assess the effectiveness of the capping operations. From November 21 to December 23, 1996, the clamshell bucket dredge supplied an estimated barge volume of $22,200 \mathrm{~m}^{3}$ of CDM for deposition over the Royal River pseudo-UDM deposit (Section 2.1). Because the outer reaches originally designated to serve as CDM were previously dredged and deposited at the DG buoy (Figure 2-9), the project design had to be modified. The dredge was positioned near the mouth of the Royal River to obtain capping material distinct from the pseudo-UDM. The sandy sediments near the outer reaches of the river were placed over the pseudo-UDM deposit as the first layers of cap, with the goal of providing an indicator of the CDM/pseudo-UDM boundary. Dredging operations proceeded up the river, with barges loading sediments from the middle and upper reaches of the river. The postcap data presented below were evaluated with respect to this dredging sequence.

\subsubsection{Bathymetry}

SAIC performed the final bathymetric survey for the Portland Disposal Site Capping Demonstration Project on January 14, 1997. Depth difference calculations based on comparisons with the November 1996 pseudo-UDM survey were used to measure the changes in bottom topography resulting from the deposition of CDM. The results indicated that, although some accumulation of $>20 \mathrm{~cm}$ was present east-southeast of the PDA buoy, the volume of CDM was too small to be confidently estimated by comparing these two surveys.

To evaluate the total thickness of the deposit, including both pseudo-UDM and CDM, the January 1997 CDM survey data and the February 1996 baseline surveys were compared, resulting in an irregularly shaped bottom feature approximately $200 \mathrm{~m}$ wide, with a maximum height of $1.5 \mathrm{~m}$ (Figure 4-21). The bathymetry data suggested that the majority of the sediment deposit accumulated $50 \mathrm{~m}$ south and east of the PDA buoy position, probably due to a consistent disposal pattern. The footprint of UDM, shown in red on Figure 4-21, generally was consistent with the location of the overall disposal mound. Discrepancies between the two surveys along the two east-west oriented lobes of material measured during the pseudo-UDM survey again suggest that these areas, 


\section{Portland Disposal Site Depth Difference \\ January 1997 CDM Survey vs. February 1996 Baseline Survey}

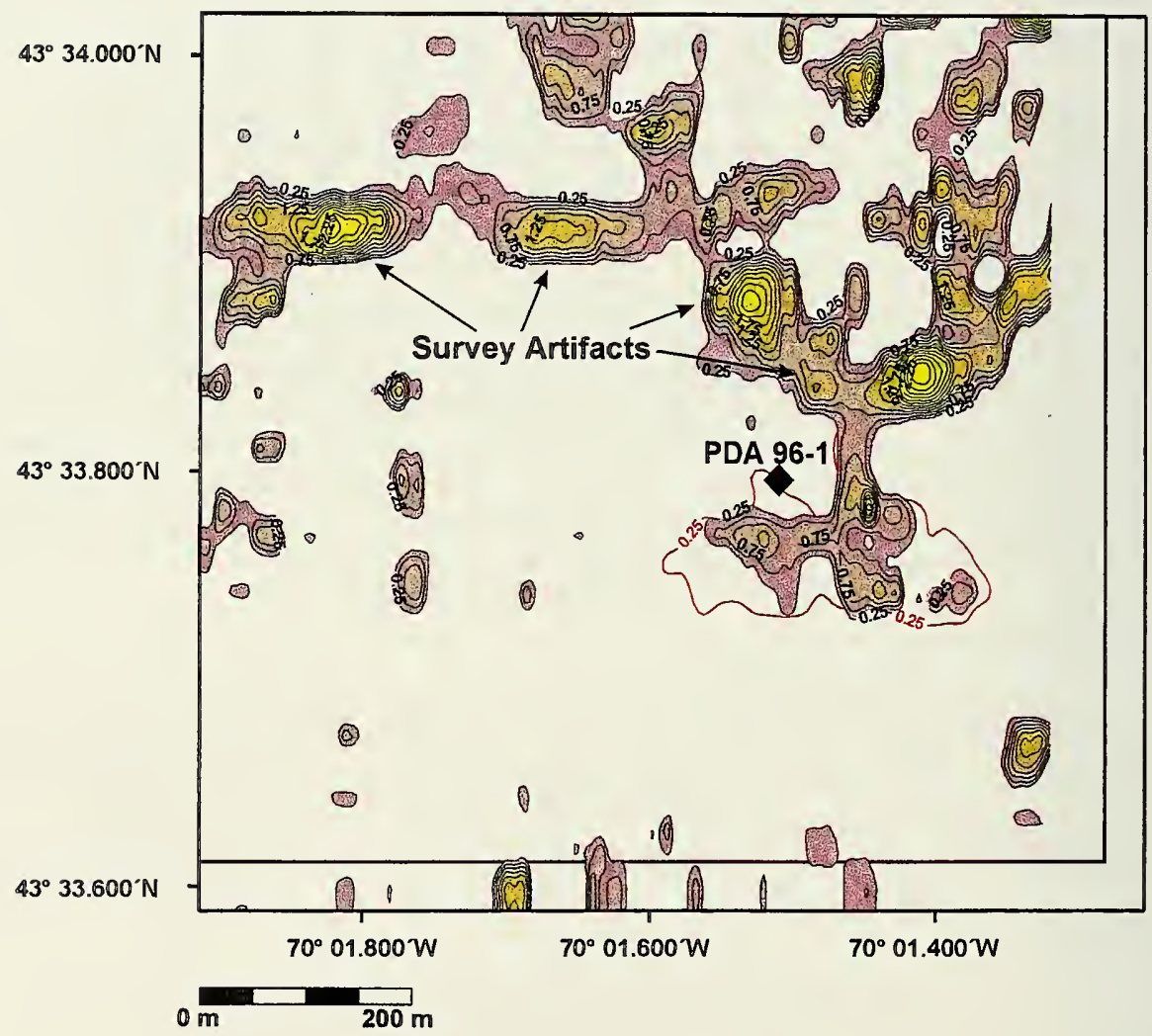

Figure 4-21. Depth difference comparison of the January 1997 CDM survey versus the February 1996 baseline survey relative to the pseudo-UDM footprint (red)

The Portland Disposal Site Capping Demonstration Project, 1995-1997 
overlying pre-existing topographic features, were affected by survey artifact. The sediment-profile data were examined closely to establish the thin stratigraphy of pseudoUDM and CDM in these areas.

\subsubsection{REMOTS ${ }^{\circledast}$ Sediment-Profile Photography}

Due to the small volume of capping material $\left(22,200 \mathrm{~m}^{3}\right)$ and the limitations of sequential bathymetric surveys over the irregular seafloor of PDS, sediment-profile photography was crucial in documenting the existence of a thin but distinct CDM layer over the Royal River pseudo-UDM deposit. A gray silt with a high sand fraction characterized the first layers of cap material extracted from the lower reaches of the river. As dredging operations proceeded up-river, a dark, homogeneous silt component became prevalent. Therefore, the presence of the CDM layer in the REMOTS ${ }^{\circledR}$ images was determined by 1) appearance of a coarser grained sand fraction within the sediment matrix; 2) detection of an RPD or an identified sedimentary sequence from the previous pseudoUDM survey buried by a homogeneous, dark layer; or 3) average dredged material thickness over ambient sediment (or historic dredged material) for a station during the CDM survey that exceeded the depth encountered during the pseudo-UDM survey.

We noted CDM deposition indicators at all stations within a $200 \mathrm{~m}$ radius of the PDA buoy position, except at 200W. As in the pseudo-UDM survey, the apparent thickness of the cap was often controlled by the depth of camera penetration (Figure 4-22). Average cap material thickness ranged from 1 to $2 \mathrm{~cm}$ at the peripheral stations to full camera penetration at CTR (Table 4-6). Mapping of the surficial sediment type and thickness indicated the CDM apron spread to a diameter $>600 \mathrm{~m}$ within the northwestsoutheast trending trough (Figure 4-22). Multiple sediment strata (CDM over pseudoUDM, or CDM over pseudo-UDM over ambient) with buried RPDs were often visible within the REMOTS ${ }^{\circledR}$ photographs obtained from the outer stations (Figure 4-23).

The coarse sand fraction of the outer channel was present at many of the 33 REMOTS $^{\circledR}$ stations, often marking the CDM/pseudo-UDM interface (Figure 4-24). However, stations near the center of the survey grid tended to display layers of the finer grained, dark silt at the surface, representative of the last stage of CDM from the middle reach (Figure 4-25). Furthermore, thicker layers of homogeneous silt, that most likely obscured the presence of a sand layer, were concentrated to the south and east of the survey grid where bathymetric changes were most pronounced.

Chunks of natural wood (limbs, branches, and bark) were visible on the seafloor and in the sediment, possibly remnants of the logging or ship building industries once prevalent on the Royal River. We also observed shell fragments within the sediments photographed by the REMOTS ${ }^{\circledR}$ camera. A large section of common razor clam (Ensis directus) shells provided further evidence of the estuarine origin of the recently deposited 


\section{Table 4-6}

January 1997 Postcap REMOTS ${ }^{\circledR}$ Survey Results

\begin{tabular}{|c|c|c|c|c|c|c|}
\hline Postcap S urvey & Replicate & \multicolumn{2}{|c|}{ Penentration Depth } & \multicolumn{3}{|c|}{ Cap Thickness } \\
\hline Station & & $(\mathrm{cm})$ & Average & $(\mathrm{cm})$ & $>$ pen* & Average \\
\hline CTR & $\mathbf{A}$ & 9 & 7.0 & 9 & >pen & 7.0 \\
\hline CTR & $\bar{B}$ & 9 & & 9 & >pen & \\
\hline CTR & $\bar{C}$ & 4 & & 4 & >pen & \\
\hline CTR & $\overline{\mathrm{D}}$ & 10 & & 10 & $>$ pen & \\
\hline$\overline{\text { CTR }}$ & $\mathbf{E}$ & 7 & & 7 & >pen & \\
\hline$\overline{\text { CTR }}$ & 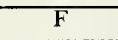 & 3 & & 3 & >pen & \\
\hline $50 \mathrm{~N}$ & $\mathbf{A}$ & 10 & 10.0 & 10 & >pen & 10.0 \\
\hline $50 \mathrm{~N}$ & $\bar{B}$ & 9 & & 9 & >pen & \\
\hline $50 \mathrm{~N}$ & $\mathrm{C}$ & 11 & & 11 & >pen & \\
\hline $100 N$ & $\bar{A}$ & 13 & 12.7 & 10 & & 9.7 \\
\hline $100 \mathrm{~N}$ & $\overline{\mathbf{B}}$ & 13 & & 10 & & \\
\hline $100 \mathrm{~N}$ & $\mathbf{C}$ & 12 & & 9 & & \\
\hline $200 \mathrm{~N}$ & $\bar{A}$ & 6 & 7.7 & 4 & & 4.0 \\
\hline $200 \mathrm{~N}$ & $\mathbf{B}$ & 8 & & 4 & & \\
\hline $200 N$ & $\overline{\mathrm{C}}$ & 9 & & 4 & & \\
\hline$\overline{50 N E}$ & $\mathbf{A}$ & 11 & 11.7 & 12 & >pen & 10.7 \\
\hline $50 \mathrm{NE}$ & B & 12 & & 12 & >pen & \\
\hline $50 \mathrm{NE}$ & $\bar{C}$ & 12 & & 8 & & \\
\hline $100 \mathrm{NE}$ & $\bar{A}$ & 13 & 13.7 & 12 & & 12.0 \\
\hline $100 \mathrm{NE}$ & $\overline{\mathbf{B}}$ & 13 & & 6 to 13 & & \\
\hline $100 \mathrm{NE}$ & $\bar{C}$ & 15 & & 10 to 15 & & \\
\hline 50ENE & $\bar{A}$ & 6 & 8.3 & 5 & & 7.0 \\
\hline $50 \mathrm{ENE}$ & $\overline{\mathbf{B}}$ & 7 & & 5 & & \\
\hline $50 \mathrm{ENE}$ & $\mathbf{C}$ & 12 & & 11 & & \\
\hline $100 \mathrm{ENE}$ & $\bar{A}$ & 12 & 11.7 & $\mathrm{NA}$ & & 9.5 \\
\hline $100 \mathrm{ENE}$ & $\overline{\mathrm{B}}$ & 13 & & 11 & & \\
\hline 100ENE & $\mathrm{C}$ & 10 & & 8 & & \\
\hline $150 \mathrm{ENE}$ & $\mathbf{A}$ & 13 & 11.7 & 11 & & 9.3 \\
\hline 150ENE & $\bar{B}$ & 14 & & 9 & 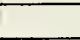 & \\
\hline $150 \mathrm{ENE}$ & $\overline{\mathrm{C}}$ & 8 & & 8 & >pen & \\
\hline 200ENE & $\bar{B}$ & 3 & 11.4 & 3 & >pen & 9.3 \\
\hline $200 \mathrm{ENE}$ & $\mathbf{C}$ & 14 & & 8 & & \\
\hline $200 \mathrm{ENE}$ & $\overline{\mathbf{D}}$ & 12 & & $\mathrm{NA}$ & & \\
\hline $200 \mathrm{ENE}$ & $\bar{E}$ & 14 & & 13 & & \\
\hline $200 \mathrm{ENE}$ & $F$ & 14 & & 13 & & \\
\hline
\end{tabular}

">pen" indicates thickness greater than the camera penetration; where not specified, ambient and dredged material were below measured cap material. 
Table 4-6 (continued)

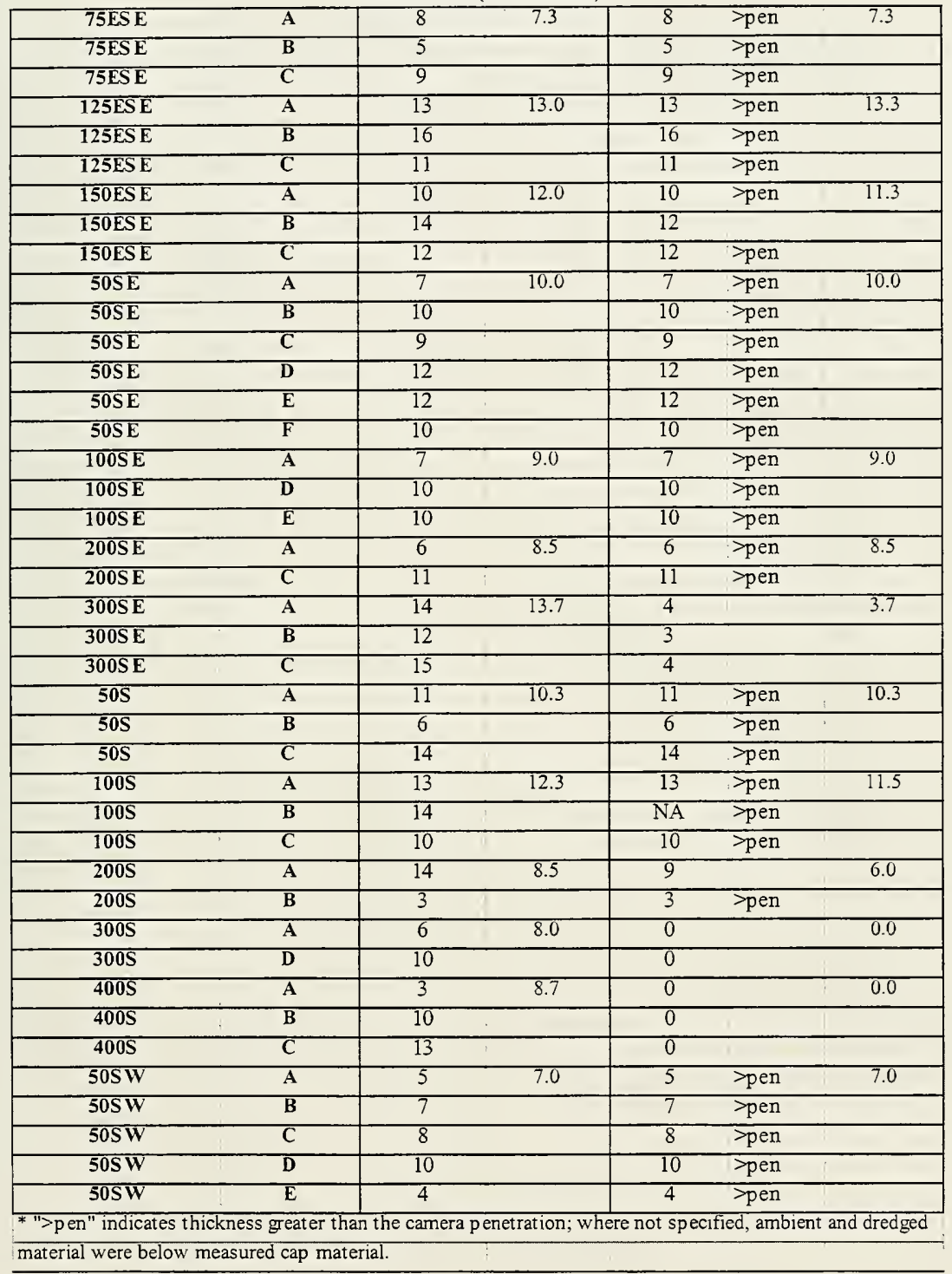


Table 4-6 (continued)

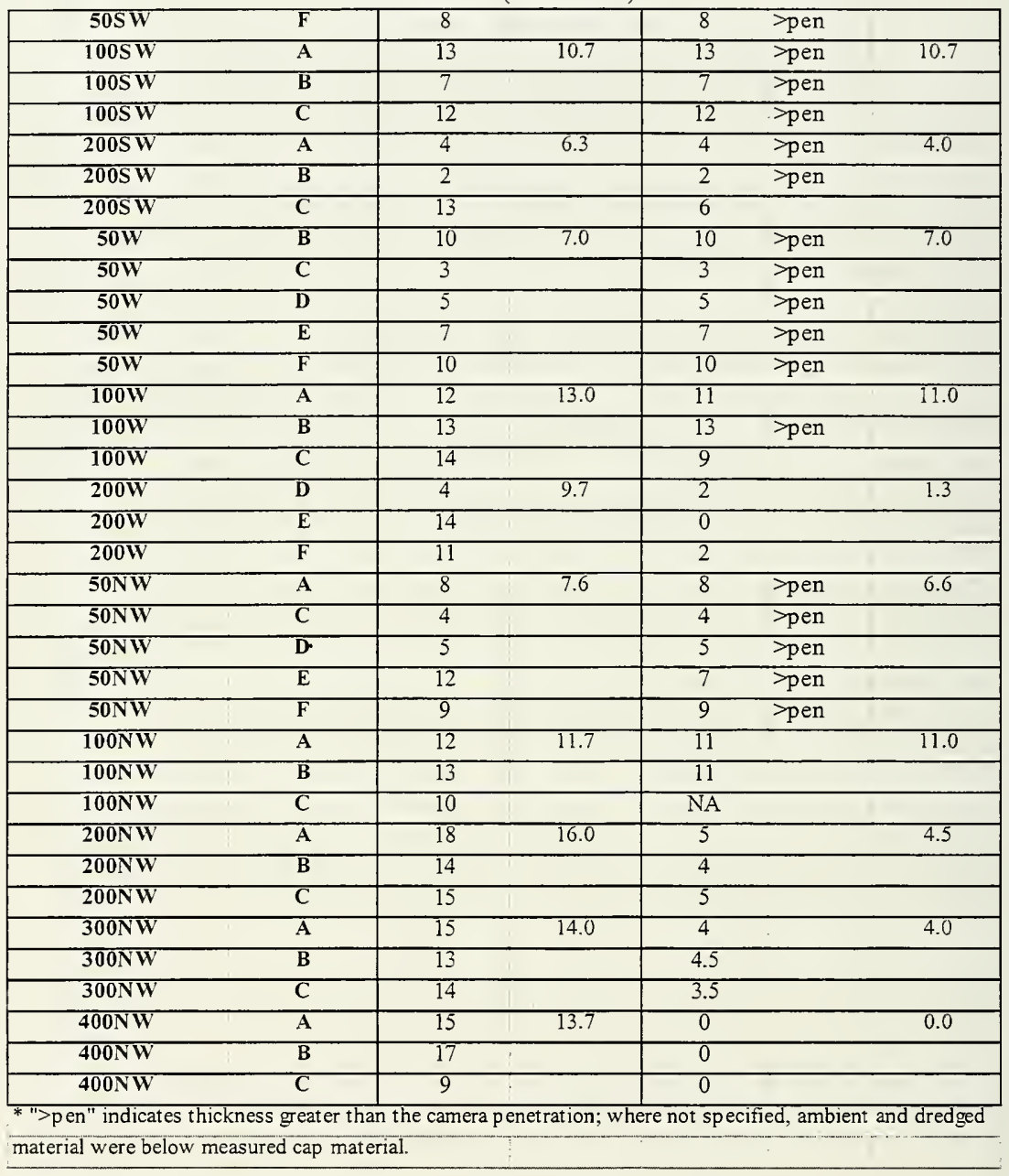




\section{Portland Disposal Site Royal River Project Area Postcap REMOTS $®$ Survey Stations over Postcap Bathymetry}

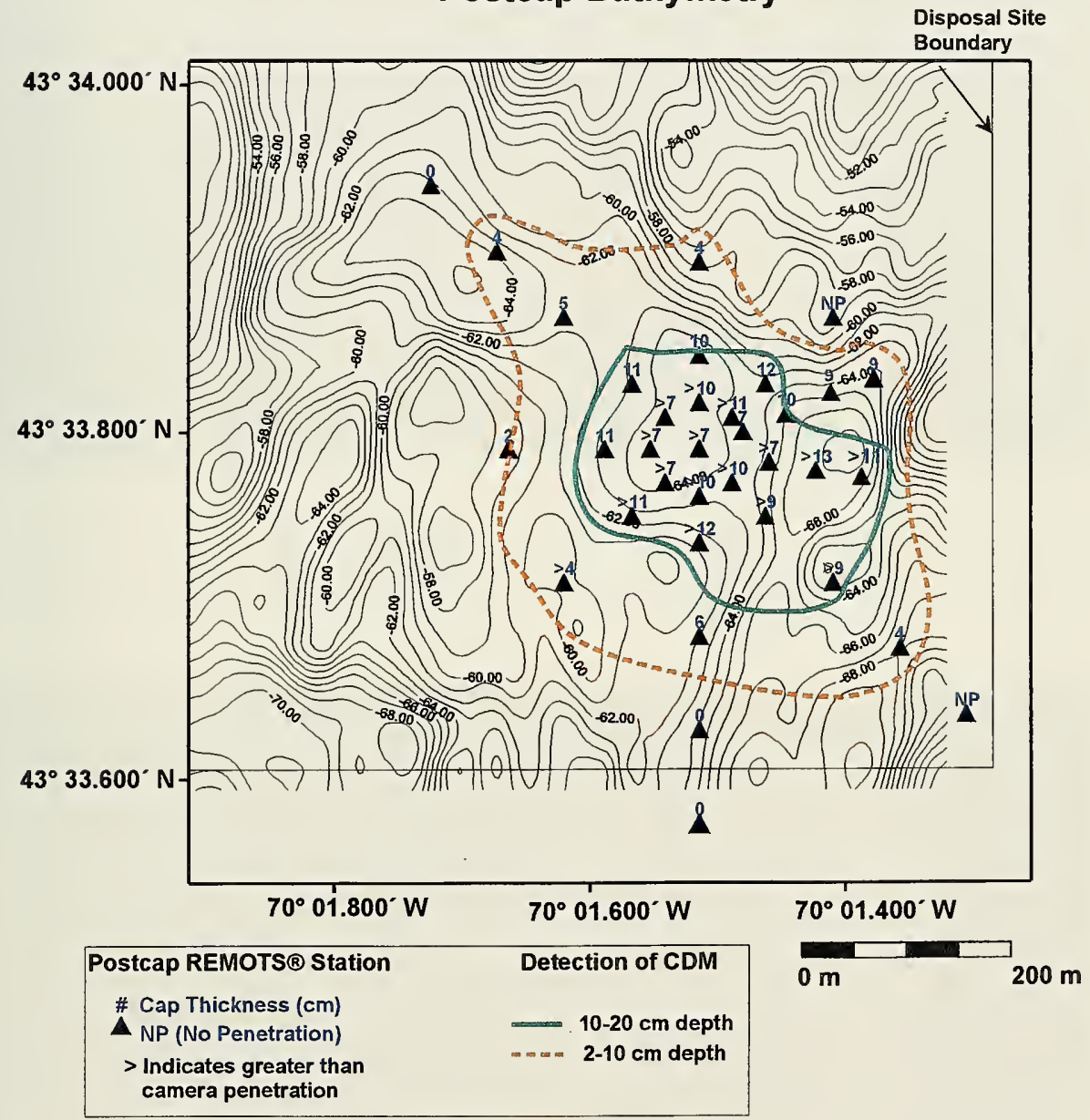

Figure 4-22. Thickness of CDM measured by REMOTS® data overlaid on postcap bathymetric chart (NAD 27), $1.0 \mathrm{~m}$ contour interval (MLLW) 

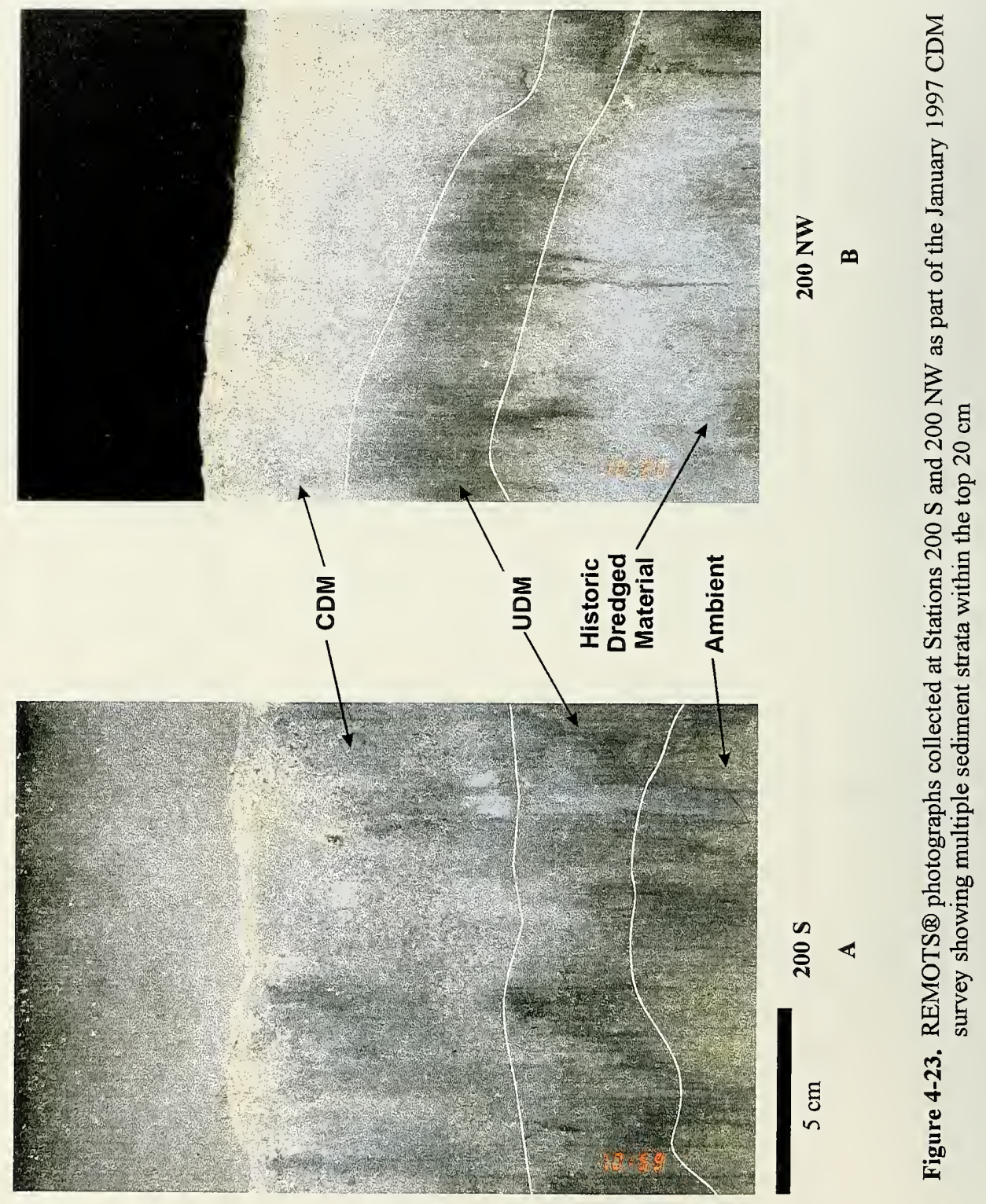

The Portland Disposal Site Capping Demonstration Project, 1995-1997 

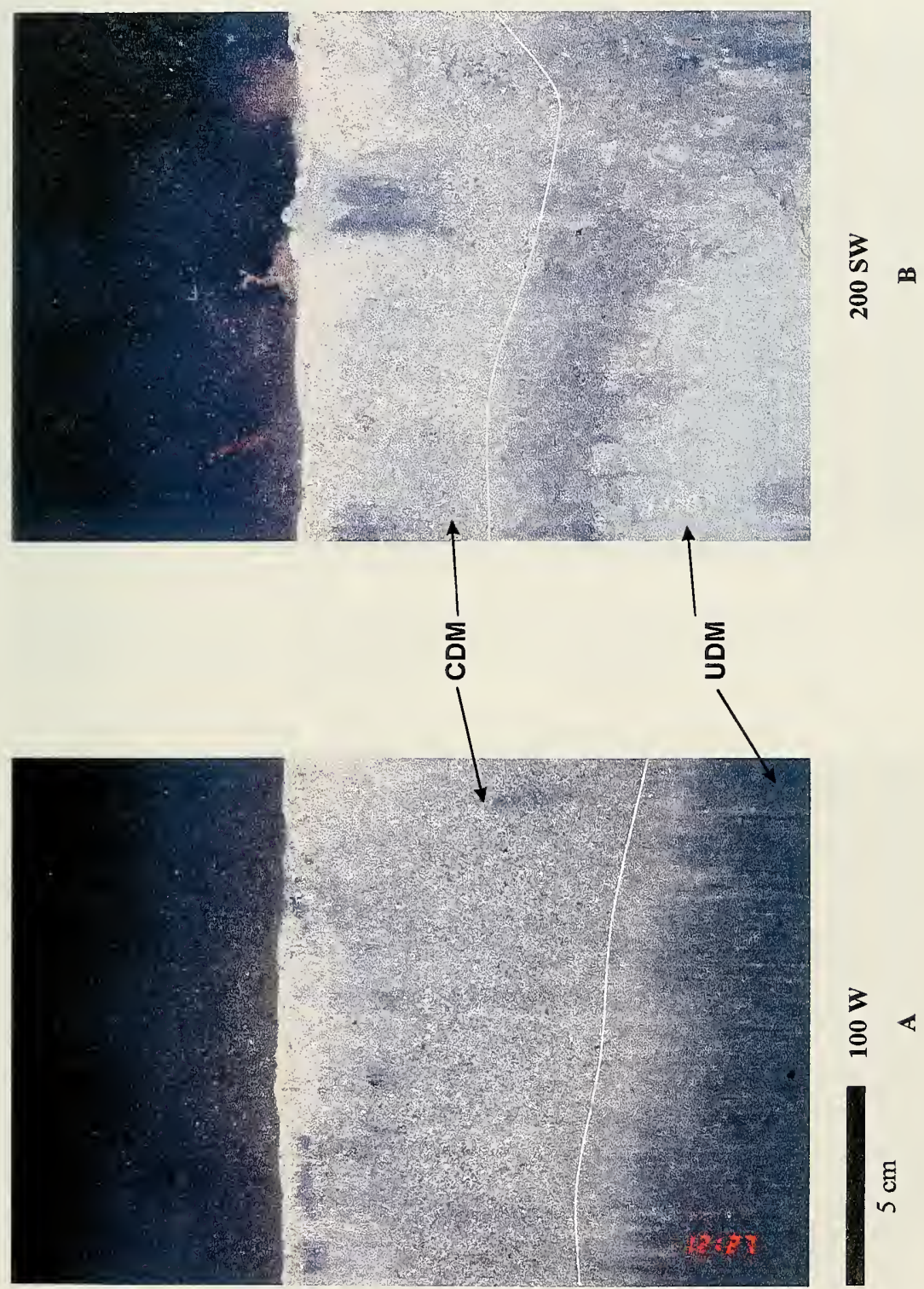

主

ลू

్ㅗㅀ

委

둘

4

㲅

8 '

귬

믕

$3 \dot{0}$

$\circ 2$

으뭄

क त

율 प्ठ
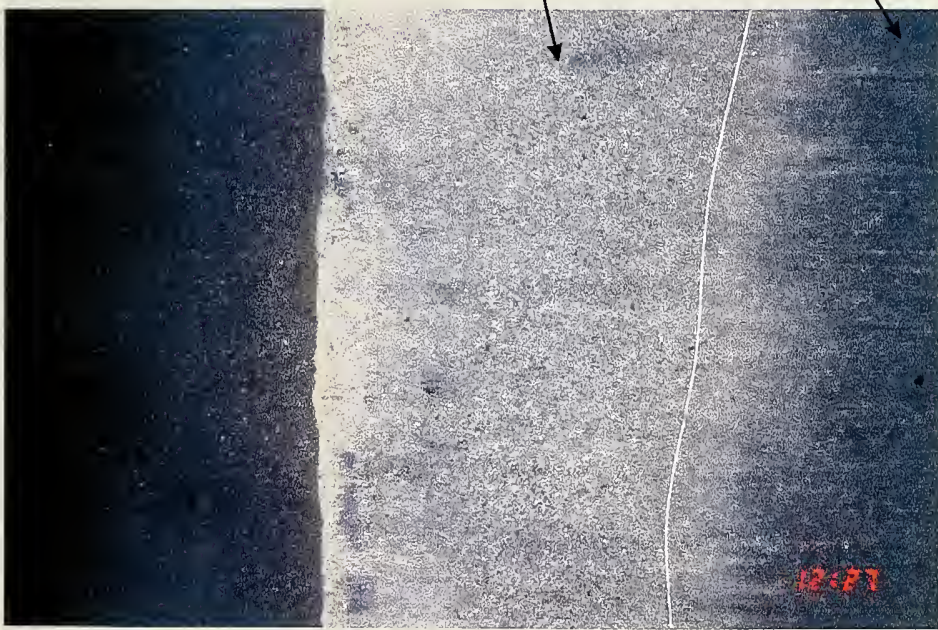

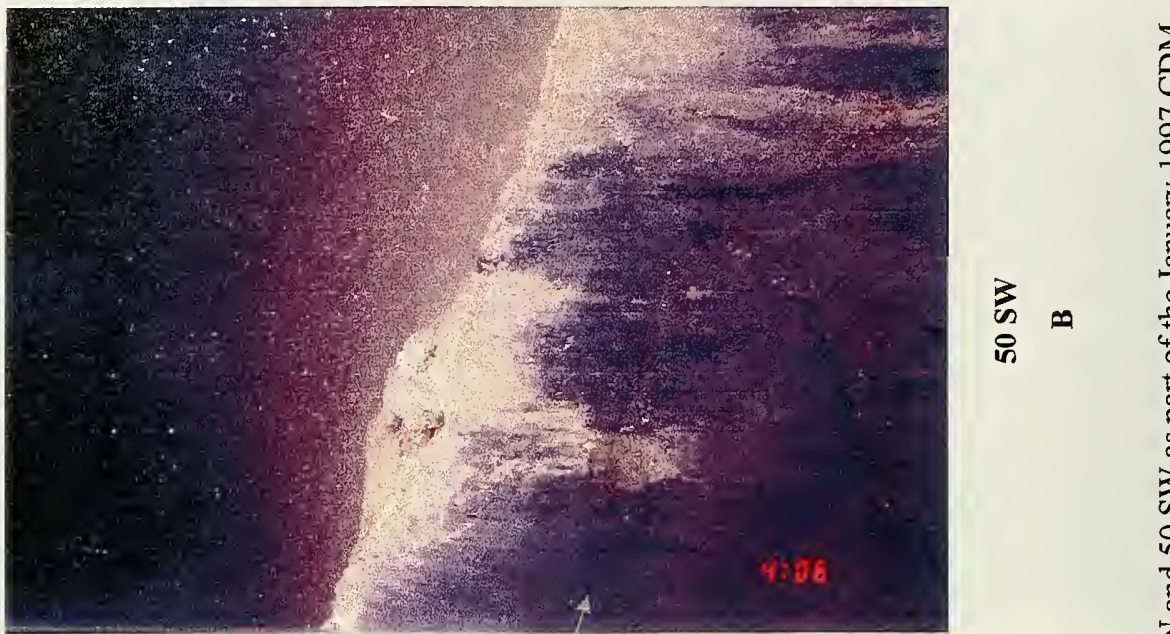

ำ

즘

인

쿠

ฉृ

की

$3 \frac{0}{0}$

욜

흥

क व

乙

으릌

is a
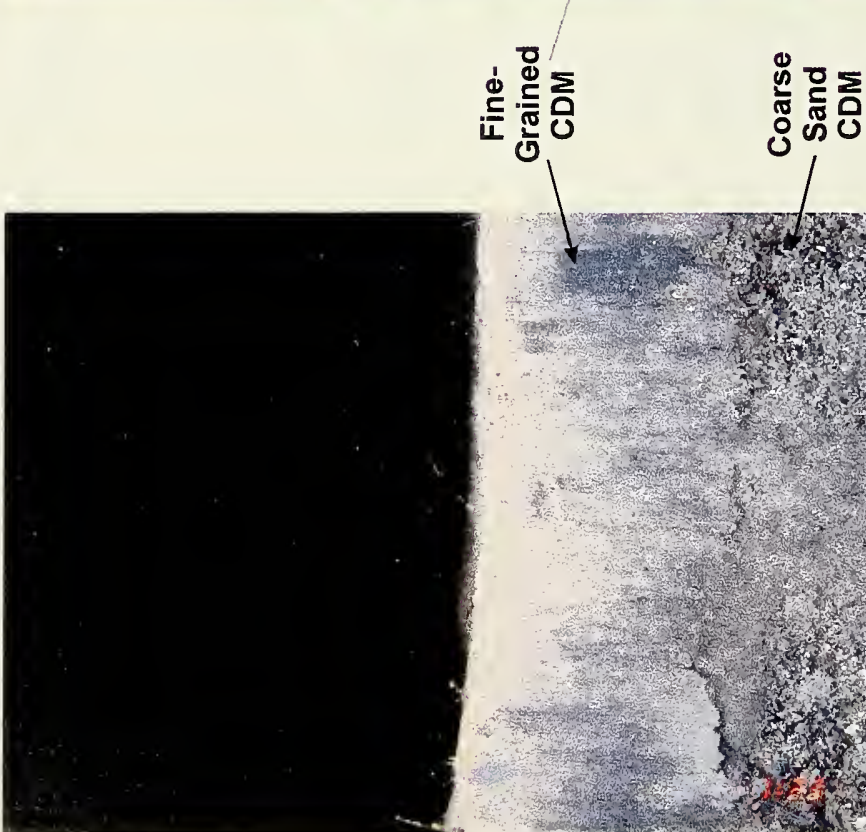

ஸे

응 농

तี

ज

호

प

용

웅

8 .

들 है
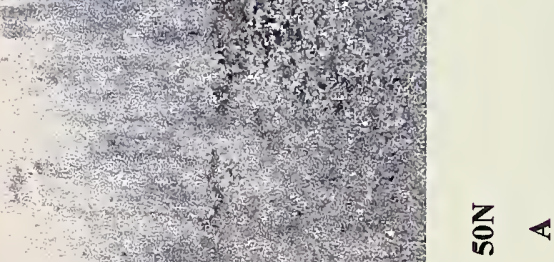

त्रि

t5

용

을 몽

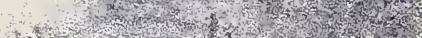

은

5 के

일

포 할

ํำ 
surficial sediment layer (Figure 4-26A). RPD depths ranging from 1 to $3 \mathrm{~cm}$ and the presence of several infaunal deposit feeding worms in the sediment-profile photographs indicated a healthy benthic recolonization of the dredged material (Figure 4-26B).

\subsubsection{Sediment Coring}

We obtained nine sediment cores from the Royal River mound during the CDM survey to provide a cross section of the CDM, pseudo-UDM, and ambient sediments (Figure 4-27). Each core was split, described, and analyzed to both supplement and verify the bathymetric and REMOTS ${ }^{\circledR}$ sediment-profile photography results. The recovered sediments were sub-sampled relative to visible sediment horizons, preserved, and analyzed for grain size and mineralogical composition, as well as microfossil content.

\subsubsection{Visual Core Descriptions}

As detected in the REMOTS ${ }^{\circledR}$ photographs, the CDM/pseudo-UDM interface was marked by a sharp change in sediment grain size. Although the dark, olive green to black color of the sediments was consistent throughout, a distinct boundary between the two sediment layers was detected in six of the nine cores collected. The thickness of CDM ranged from $9 \mathrm{~cm}$ in Core $\mathrm{G} 2$ to $36 \mathrm{~cm}$ in Core E6. Cores F2 and $\mathrm{H} 2$ were excluded from analysis, because the cores yielded only a small volume of material which increased the likelihood of disturbance during retrieval.

Ambient sediments, characterized as a medium-grained sand (1.0 to 2.0 phi), were recovered in the bottom of Cores A9 and G2. A similar tan, sandy component was collected near the bottom of Core $\mathrm{C} 1$. However, the material in $\mathrm{C} 1$ appeared to be stratified by grain size and emitted a strong smell of hydrogen sulfide $\left(\mathrm{H}_{2} \mathrm{~S}\right)$, possibly linking it to the sewer outfall in Royal River or the 1995 improvement dredging project at a marina in the Harraseeket River. Shell and wood fragments were found throughout all nine sediment cores. Complete descriptions and graphical representations based on the visual descriptions of the CDM sediment cores can be found in Appendix C.

\subsubsection{Core Grain Size Analysis}

An analysis of the ranges of grain size was made based on the visual material classifications (Section 4.5.3.1). In general, grain size analysis of the samples extracted from Cores A9 through I1 supported the presence of three distinct layers of material (CDM, pseudo-UDM, and ambient) over the disposal mound (Table 4-7). These data are evaluated below, and compared with the grain size data collected in the grab samples collected following placement of the pseudo-UDM. 

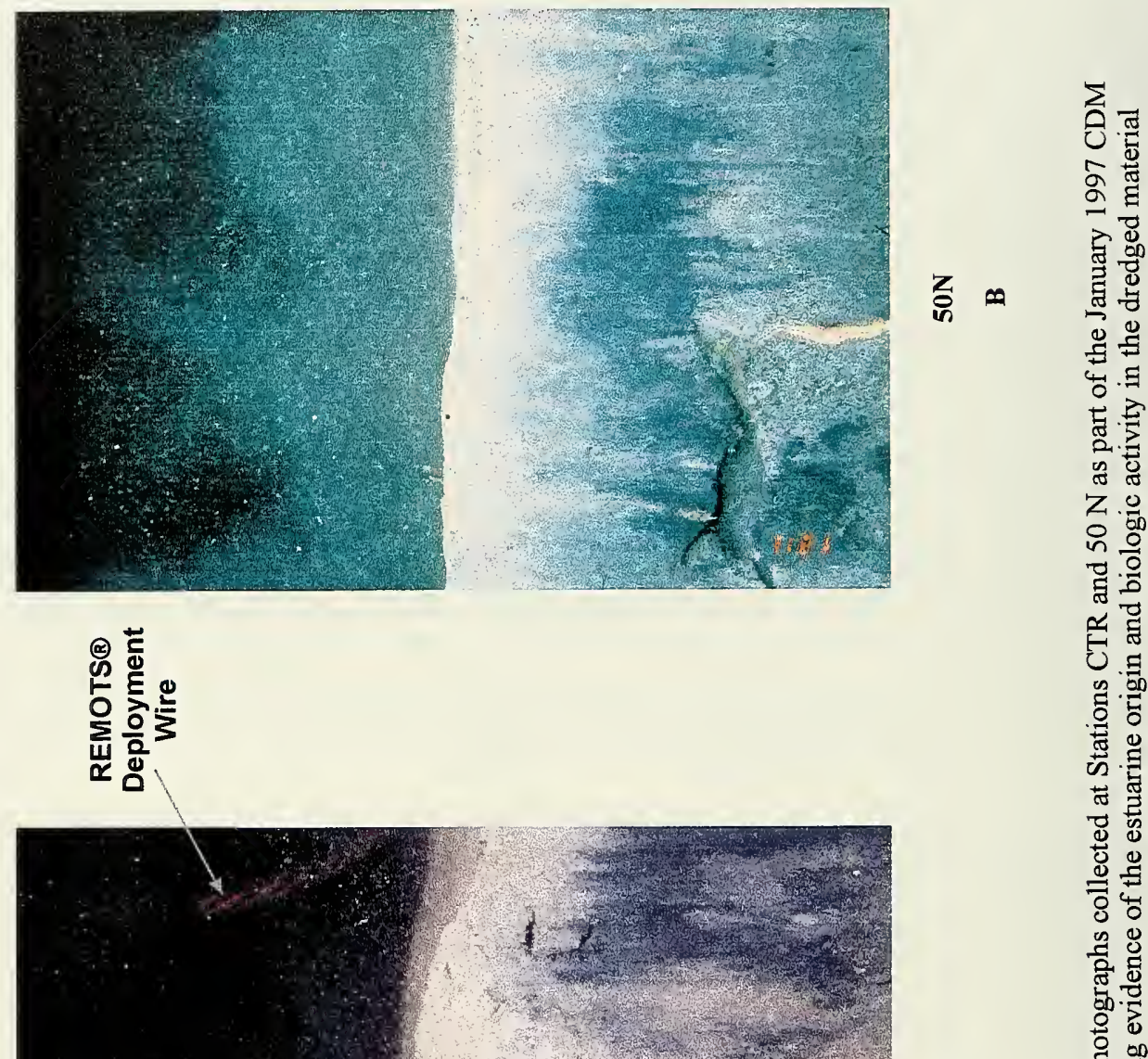


\section{Portland Disposal Site \\ Royal River Project Area Depth Difference \\ Target Geotechnical Coring Locations over Apparent Accumulation of Pseudo-UDM and CDM}

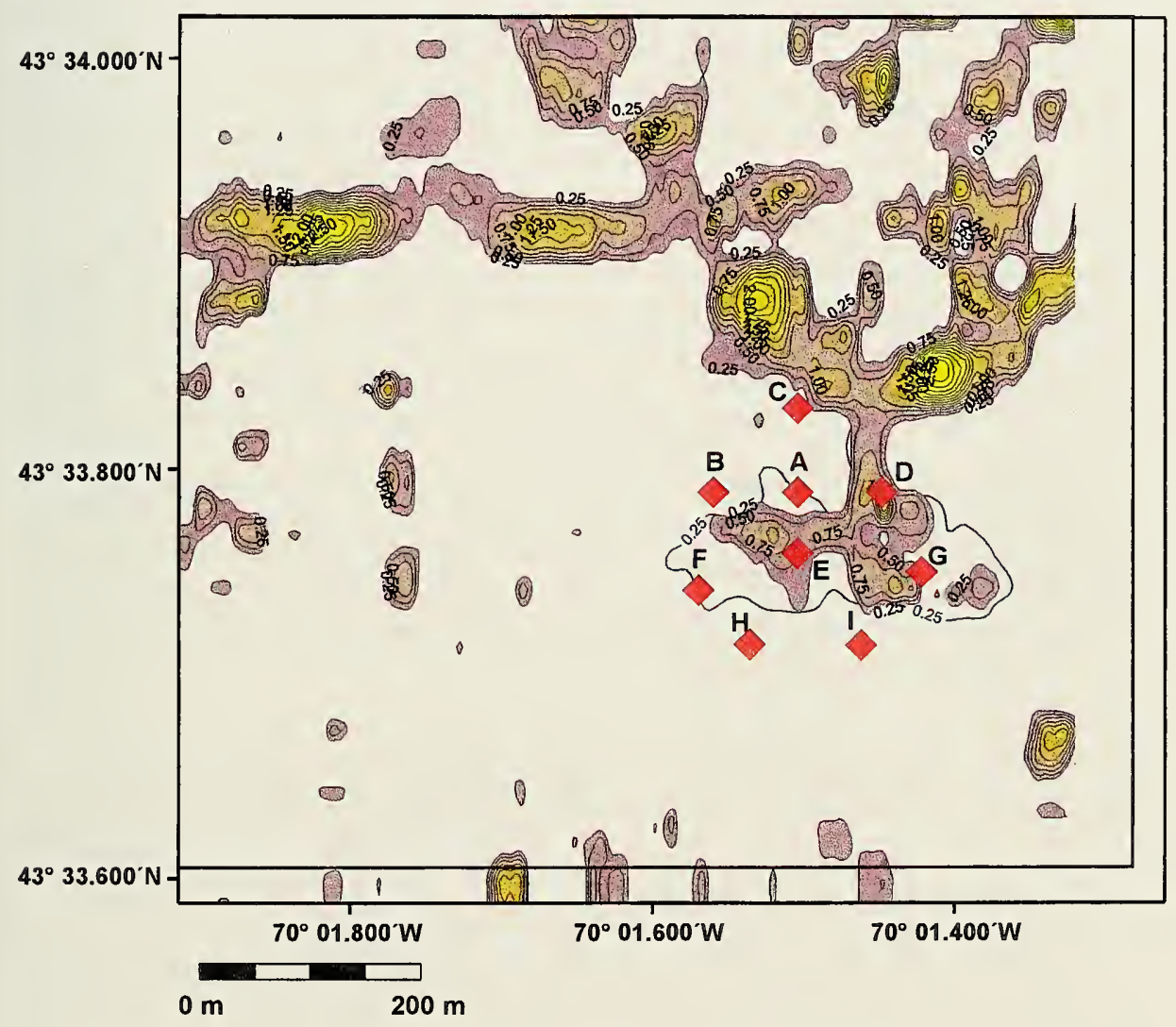

Figure 4-27. Depth difference plot showing detectable capped mound deposit (NAD 27) complete with gravity coring station locations 
Table 4-7

Postcap (CDM) Sediment Coring Survey Grain Size Analysis Results

\begin{tabular}{|c|c|c|c|c|}
\hline Core & Depth (cm) & \%Sand & \%Silt & \%Clay \\
\hline A-9 & $6-10 \mathrm{~cm}$ & 40.6 & 42.8 & 16.5 \\
\hline A-9 & $16-20 \mathrm{~cm}$ & 5.3 & 57.7 & 37.0 \\
\hline A-9 & $30-34 \mathrm{~cm}$ & 62.7 & 24.7 & 12.6 \\
\hline A-9 & $39-43 \mathrm{~cm}$ & 11.9 & 65.2 & 22.9 \\
\hline A-9 & $48-52 \mathrm{~cm}$ & 19.2 & 55.0 & 25.8 \\
\hline A-9 & $53-57 \mathrm{~cm}$ & 52.1 & 30.3 & 17.6 \\
\hline \hline B-1 & $3-7 \mathrm{~cm}$ & 64.3 & 24.1 & 11.6 \\
\hline B-1 & $10-14 \mathrm{~cm}$ & 83.2 & 8.9 & 7.9 \\
\hline B-1 & $18-22 \mathrm{~cm}$ & 37.2 & 39.0 & 23.8 \\
\hline B-1 & $24-31 \mathrm{~cm}$ & 56.3 & 27.8 & 15.8 \\
\hline \hline C-1 & $0-4 \mathrm{~cm}$ & 36.4 & 46.3 & 17.3 \\
\hline C-1 & $7-11 \mathrm{~cm}$ & 57.2 & 29.4 & 13.4 \\
\hline C-1 & $15-19 \mathrm{~cm}$ & 35.0 & 42.7 & 22.3 \\
\hline C-1 & $26-30 \mathrm{~cm}$ & 26.1 & 32.5 & 41.4 \\
\hline C-1 & $40-44 \mathrm{~cm}$ & 81.2 & 11.3 & 7.5 \\
\hline C-1 & $53-57 \mathrm{~cm}$ & 88.7 & 6.5 & 4.8 \\
\hline \hline D-2 & $2-6 \mathrm{~cm}$ & 82.1 & 12.6 & 5.3 \\
\hline D-2 & $8-12 \mathrm{~cm}$ & 70.7 & 22.1 & 7.2 \\
\hline D-2 & $19-23 \mathrm{~cm}$ & 12.4 & 63.2 & 24.4 \\
\hline D-2 & $25-29 \mathrm{~cm}$ & 17.6 & 57.2 & 25.2 \\
\hline D-2 & $34-37 \mathrm{~cm}$ & 4.7 & 64.6 & 30.7 \\
\hline \hline E-6 & $2-6 \mathrm{~cm}$ & 16.5 & 66.3 & 17.2 \\
\hline E-6 & $8-12 \mathrm{~cm}$ & 24.0 & 56.5 & 19.5 \\
\hline E-6 & $18-22 \mathrm{~cm}$ & 60.3 & 26.0 & 13.8 \\
\hline E-6 & $30-34 \mathrm{~cm}$ & 62.9 & 25.0 & 12.1 \\
\hline E-6 & $40-44 \mathrm{~cm}$ & 52.8 & 32.5 & 14.8 \\
\hline E-6 & $45-49 \mathrm{~cm}$ & 21.0 & 57.5 & 21.4 \\
\hline E-6 & $50-54 \mathrm{~cm}$ & 8.4 & 66.3 & 25.2 \\
\hline E-6 & $55-60 \mathrm{~cm}$ & 9.3 & 64.8 & 25.9 \\
\hline E-6 & $65-69 \mathrm{~cm}$ & 7.3 & 64.2 & 28.5 \\
\hline \hline G-2 & $4-8 \mathrm{~cm}$ & 61.9 & 26.2 & 11.9 \\
\hline G-2 & $10-13 \mathrm{~cm}$ & 30.5 & 48.9 & 20.6 \\
\hline G-2 & $24-28 \mathrm{~cm}$ & 33.8 & 45.3 & 20.8 \\
\hline G-2 & $32-36 \mathrm{~cm}$ & 75.5 & 12.4 & 12.2 \\
\hline G-2 & $52-56 \mathrm{~cm}$ & 91.4 & 4.4 & 4.2 \\
\hline \hline I-1 & $0-4 \mathrm{~cm}$ & 64.4 & 23.0 & 12.6 \\
\hline I-1 & $8-12 \mathrm{~cm}$ & 79.0 & 14.3 & 6.7 \\
\hline I-1 & $15-19 \mathrm{~cm}$ & 84.1 & 10.3 & 5.6 \\
\hline I-1 & $22-26 \mathrm{~cm}$ & 47.0 & 37.9 & 15.2 \\
\hline UDM & $27-28 \mathrm{~cm}$ & 47.2 & 36.9 & 16.0 \\
\cline { 2 - 5 } AMB & $40-44 \mathrm{~cm}$ & 21.2 & 53.8 & 25.0 \\
\hline & Average & 27.6 & 50.2 & 22.2 \\
\hline Ave & 72.6 & 13.4 & 14.0 \\
\hline
\end{tabular}

\begin{tabular}{|c|c|c|c|c|}
\hline Core & Depth $(\mathrm{cm})$ & \%Sand & \%Silt & \%Clay \\
\hline $\mathrm{F}-2$ & $4-8 \mathrm{~cm}$ & 22.7 & 54.9 & 22.4 \\
\hline$F-2$ & $14-18 \mathrm{~cm}$ & 26.8 & 34.9 & 38.3 \\
\hline $\mathrm{F}-2$ & $22-26 \mathrm{~cm}$ & 46.5 & 38.7 & 14.8 \\
\hline H-2 & $2-6 \mathrm{~cm}$ & $\overline{35.7}$ & 44.7 & 19.6 \\
\hline $\mathrm{H}-2$ & $9-13 \mathrm{~cm}$ & 15.0 & 49.6 & 35.4 \\
\hline \multicolumn{5}{|c|}{$\begin{array}{l}\text { Cores } \mathrm{H} \text { and } \mathrm{F} \text { were not used to calculate the } \\
\text { averages for each layer due to unreliable } \\
\text { stratigraphies and waterlogging. }\end{array}$} \\
\hline $\begin{array}{l}\text { The avera } \\
\text { seven core } \\
\text { visual obs }\end{array}$ & tions and the & from san & & \\
\hline
\end{tabular}


Samples identified as being collected in CDM and extracted from the top sections of the cores collected after cap material was placed tended to contain a relatively high sand fraction, with a mean percent composition of $56 \%$ (Figure 4-18). In general, the average silt (30\%) and clay (13\%) content of the material classified as CDM were lower than the average measured values in both the material classified as UDM in the cores (50\% and $22 \%$ silt and clay, respectively), and in the UDM grab samples (49\% and $23 \%$, respectively). However, the top CDM samples of Cores C1, E6, and A9 had grain sizes more comparable to the pseudo-UDM than the average CDM sample. This discrepancy is likely due to the deposition of the finer grained material from the middle zone of the river over the initial sandy CDM layer. The clay fraction of both the UDM samples from the cores and grab samples was consistently around $20 \%$.

Some discrepancies in these trends of grain size were noted. In the lower part of Core B $(24 \mathrm{~cm}$ to $31 \mathrm{~cm})$, the percentage of sand $(56 \%)$ within the layer classified as pseudo-UDM was exceptionally high, probably due to the incorporation of some amount of ambient material. Sand was also the dominant component in the lowest pseudo-UDM sample of Core A9 (ambient origin) and in the top pseudo-UDM sample of Core E6 (CDM origin). These discrepancies were evaluated further during statistical analyses.

The ambient samples were composed dominantly of sand (73\%). Silt had a significantly lower abundance (13\%) than observed in both the pseudo-UDM and CDM layers. The low percentage of clay $(14 \%)$ in the ambient material was comparable to the average measured in samples classified as CDM (13\%). One sample from the $26 \mathrm{~cm}$ to 30 $\mathrm{cm}$ horizon in Core $\mathrm{C} 1$, classified as pseudo-UDM (or possibly ambient) in the visual descriptions, had an unusually low percentage of sand (26\%).4.5.3.3 Fine Fraction Analysis

In eight of nine cores, distinctions between the CDM, pseudo-UDM, and ambient material were apparent based on mineralogical composition and microfossil assemblage and density.

Mineralogy. The mineralogical analysis of the fine fraction (63 $\mu \mathrm{m}$ to $500 \mu \mathrm{m})$ for the 41 samples extracted from seven of the nine PDS gravity cores indicated the composition of the sediments was predominantly quartz, with micas common. The mineralogical abundance of 16 other components of the Royal River sediment samples was used to assist in the differentiation of the three sediment layers.

The fine fraction grain size was determined to be medium-coarse for the ambient and pseudo-UDM layers and coarse for the CDM layers (Table 4-8; Figure 4-28). Due to the homogeneous nature of the material and differences in sedimentation processes, the ambient layer had the lowest overall abundance of mineralogic and biologic components 
(insect parts, plant fragments, etc.). However, this layer did have the highest abundance of planktonic diatoms, dark minerals, and rock fragments. Smooth and textured ostracods were rare, and flyash, fibrous minerals, plant fragments, and pellets were all very rare.

Similar to the grab samples obtained over the pseudo-UDM deposit in November 1996, the pseudo-UDM layer within the cores had a high abundance of plant material, black porous materials, and planktonic diatoms (Figure 4-28). Dark minerals, rock fragments, smooth and textured ostracods, and pellets were commonly present in samples, but in lesser concentrations. Flyash, fibrous minerals, insect parts, benthic diatoms, and shell fragments were rare. Although very rare, gastropod and bryozoan fragments were identified within the fine fraction.

The CDM layer had a noticeably coarser grained material in the fine fraction, as well as an abundance of black porous material. Flyash, fibrous minerals, and plant fragments were all common. Textured ostracods and pellets were present. Dark minerals, rock fragments, insect parts, smooth ostracods, and shell fragments were rare. Benthic diatoms and bryozoan parts were very rare.

Microfossils. The mean relative abundance of the five microfossil groups (freshwater thecamoebians, marsh foraminifera [agglutinated], mudflat foraminifera [calcareous], shelf agglutinated foraminifera, and shelf calcareous foraminifera) was calculated for each sample. The relative abundance is the actual number of individuals counted per sample divided by the total number of individuals. Because the abundance values are relative values, the population density, in units of numbers of microfossils per gram of sediment, also were used to characterize the layers within the cores. Results from individual cores are provided in Appendix B, and full histograms for each core are provided in Appendix D.

After the microfossil analysis, using the classification defined from the visual core descriptions, the average relative abundance for each layer (CDM, pseudo-UDM, and ambient (AMB) was calculated. As discussed below, several samples were re-classified after microfossil analysis, so that the final values for mean abundance were calculated using a total of 41 samples: five for AMB, 19 for pseudo-UDM, and 17 for CDM.

The AMB samples had the highest mean abundance of shelf agglutinated foraminifera $(74 \%)$ and shelf calcareous foraminifera (13\%), and the lowest relative abundance, and density, of all the other groups (Figure 4-29A). Samples from both Cores $\mathrm{C}$ and $\mathrm{G}$ contained marsh foraminifera in samples collected from AMB. In Core $\mathrm{C}$, the top two samples of the ambient layer, $26 \mathrm{~cm}$ to $44 \mathrm{~cm}$ deep, contained over $80 \%$ shelf foraminifera in each sample. The samples were reclassified as ambient, rather than pseudo-UDM as in the original core descriptions, but may actually have represented historic dredged material. The presence of historical dredged material within the project 
Table 4-8

Postcap Core Sample Fine Fraction Mineralogy Results

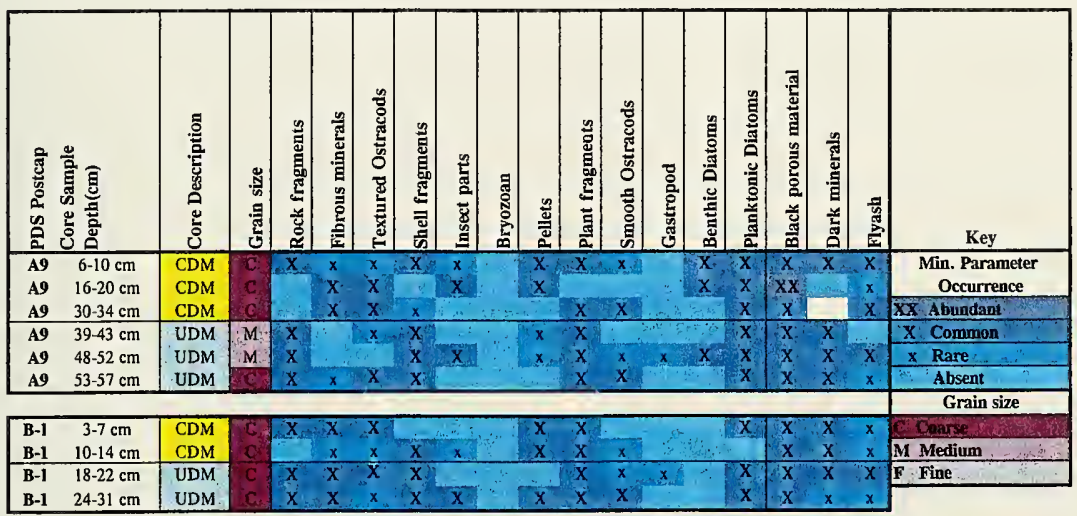

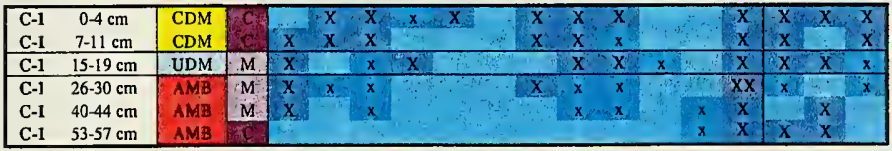

\begin{tabular}{|c|c|c|c|c|c|c|c|c|c|c|c|c|c|}
\hline D-2 & $2-6 \mathrm{~cm}$ & CDM & c & $x=x$ & & & & & & & 3 & $\bar{x}$ & \\
\hline D-2 & $8-12 \mathrm{~cm}$ & $\mathrm{CDM}$ & C. & $x \quad x$ & $x$ & $\mathrm{X}$ & $x$ & $x$ & & & & $\underline{X}$ & \\
\hline D-2 & $19-23 \mathrm{~cm}$ & UDM & M & $x \quad x$ & & $x$ & $x$ & $x$ & $\mathrm{x}$ & $\mathrm{x}$ & $x$ & $x$ & $x$ \\
\hline D-2 & $25-29 \mathrm{~cm}$ & UDM & $\mathrm{M}$ & $x$ & & $x$ & $x$ & $\mathrm{x}$ & & $x$ & $\mathrm{x}$ & $x$ & $x$ \\
\hline D-2 & $34-37 \mathrm{~cm}$ & UDM & C? & & $x$ & & $\mathrm{x}$ & $\mathrm{x}$ & & $x$ & $x$ & & $x$ \\
\hline
\end{tabular}

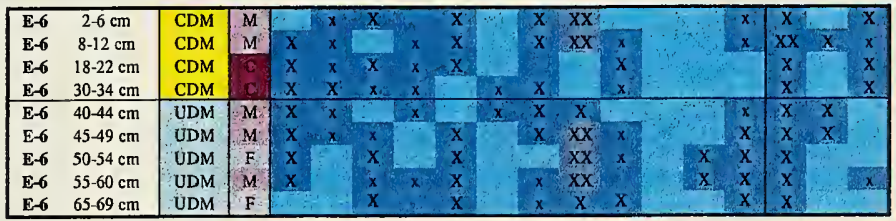

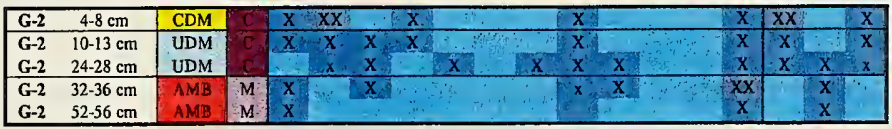

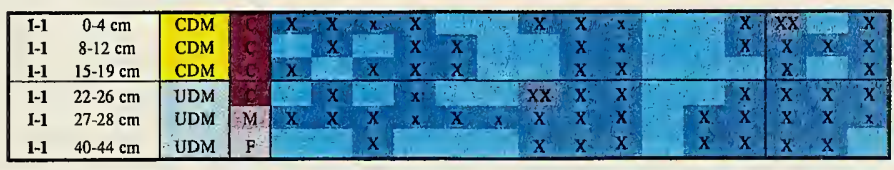




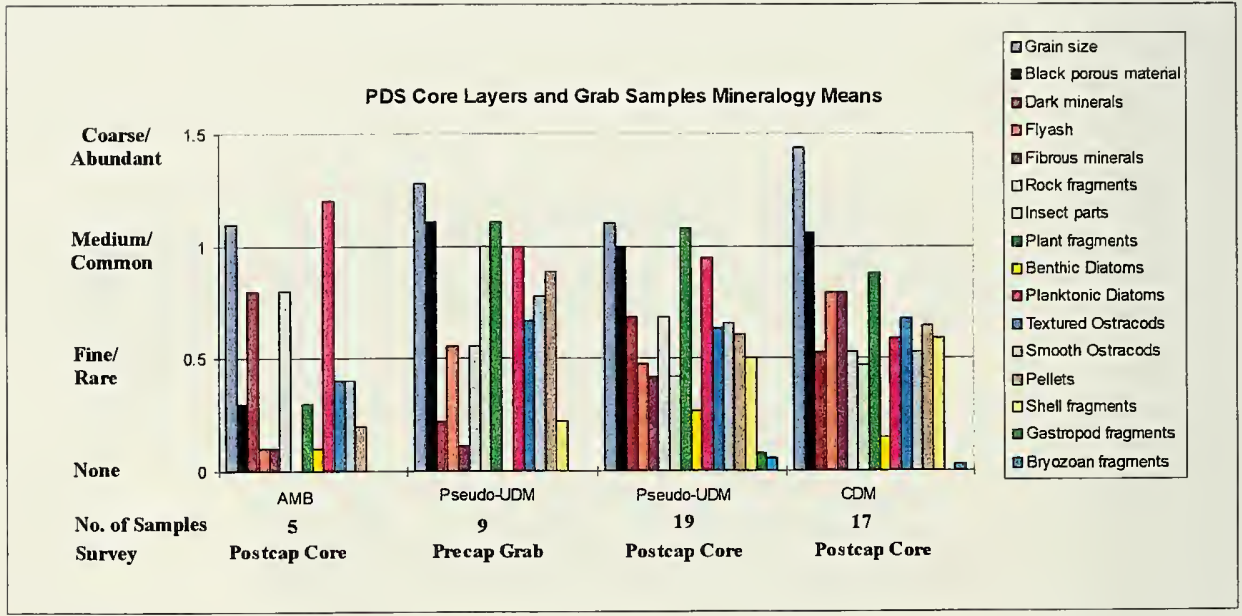

Figure 4-28. Histogram comparing the mean abundance (rare, common, abundant) and particle size (fine, medium, coarse) for the mineralogical components of the grab and core samples (CDM, pseudo-UDM, and ambient layers) 
boundaries to some extent complicated the interpretation of the monitoring data (Section 5.0). The top AMB sample in Core G contained a small amount of thecamoebians and mudflat and marsh foraminifera in the AMB unit at a depth of 31 to $57 \mathrm{~cm}$.

The pseudo-UDM samples from the postcap cores had the highest mean value of freshwater thecamoebians (19\%), with a range of $7 \%$ to $35 \%$ (Figure 4-29A). The average was consistent with data collected from the grab samples, which had the second highest thecamoebian content (approximately $15 \%$ ). The density of the thecamoebians was also highest in the pseudo-UDM (18.7 per gram), relative to 4.8 per gram in the CDM and approximately 2.0 per gram in the AMB (Figure 4-29B). The deepest sample in Core A, collected from approximately $55 \mathrm{~cm}$ down in the core, resulted in over $20 \%$ thecamoebians and no shelf foraminifera, so although this sample was originally classified as ambient during core descriptions, the sample was re-classified as pseudo-UDM. The appearance of this material, described as ambient, suggested that the sample may actually be historical dredged material, rather than dredged material associated with this project.

Both the UDM core and grab samples had a small percentage of shelf calcareous foraminifera, but no shelf agglutinated foraminifera. Shelf calcareous foraminifera were also noted in the CDM, but with a smaller relative abundance. Just above and below the $\mathrm{CDM} /$ pseudo-UDM boundary in most of the PDS cores a small percentage (2\% to $8 \%$ ) of shelf foraminifera were present. The presence of shelf foraminifera in both pseudo-UDM and CDM material may have originated from the source areas, as small numbers of shelf species were noted in the Royal River cores. However, due to their presence in the grab samples collected from the pseudo-UDM mound, the occurrence of shelf species also may have resulted from recolonization of the surficial sediment layer following disposal.

The CDM layer contained a higher percentage of both marsh and mudflat foraminifera than the pseudo-UDM layer, and had the lowest abundance $(<1 \%)$ of shelf calcareous foraminifera. Overall, the top CDM sample had the highest abundance of foraminifera. The abundances of foraminifera appeared to decrease with depth within each layer. The density of foraminifera was slightly higher in the CDM than in the pseudoUDM. The greatest abundance of foraminifera occurred consistently in the top CDM or top pseudo-UDM core sample, suggesting similarity in material composition and origin or recolonization.

Upon processing, the stratigraphy of three cores $(\mathrm{B}, \mathrm{F}$, and $\mathrm{H})$ appeared slightly disturbed and waterlogged. Although a detectable CDM/pseudo-UDM interface was visible in Core B, a small percentage of shelf foraminifera occurred in all four samples which indicated possible mixing of layers during core retrieval or transport. Core $\mathrm{H} 2$ was very short $(20 \mathrm{~cm})$, and did not have a clear division of dredged material layers based on microfossil content. Because of the clear dredged material layers, Core B was incorporated into the statistical analysis (Section 4.7), but Cores $\mathrm{F}$ and $\mathrm{H}$ were excluded. 


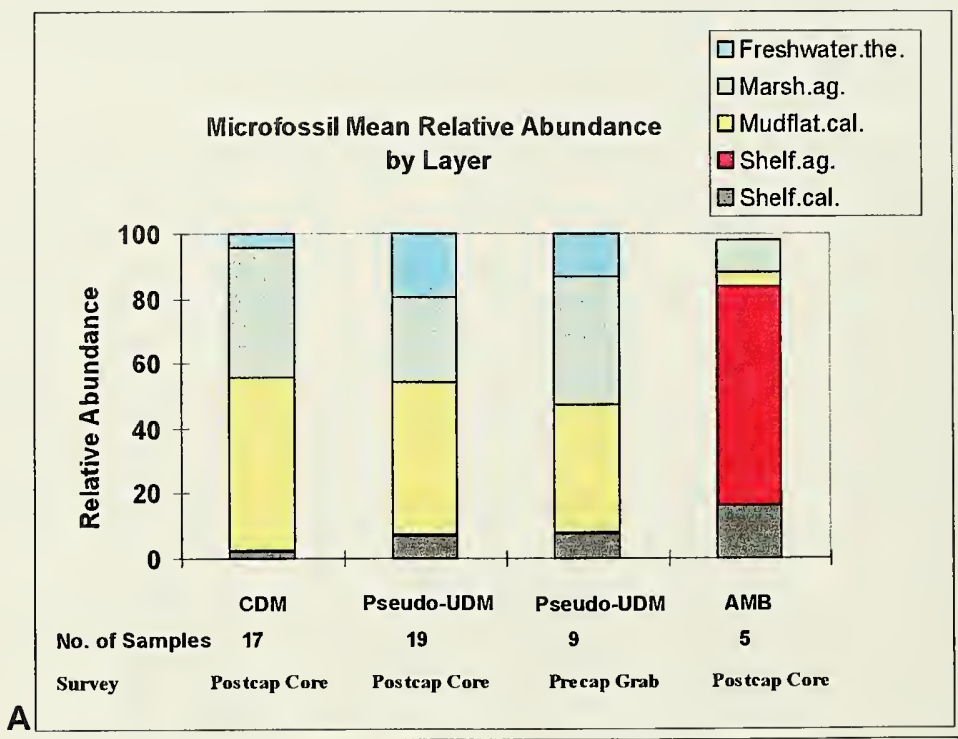

A

Mean No. per Gram

by Layer

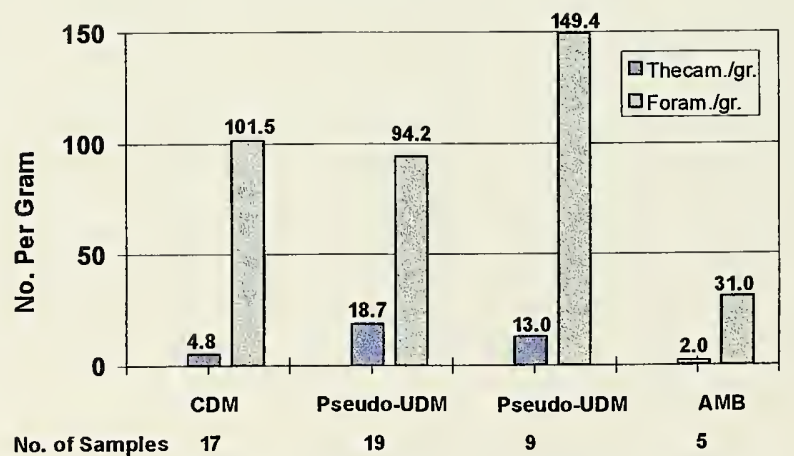

B

Survey

Postcap Core

Postcap Core

Pre cap Grab Postcap Core

Figure 4-29. Histograms comparing the mean relative abundance and density of microfossils in the sediment samples analyzed as part of the Portland Disposal Site Capping Demonstration Project

The Portland Disposal Site Capping Demonstration Project, 1995-1997 


\subsection{Processing and Analysis of Additional Royal River Cores}

To clarify the distinction between the pseudo-UDM dredged from the anchorage of the Royal River, and the CDM dredged from the middle reach, three more cores from the Royal River were processed and analyzed. Three cores (RR-6, RR-5, and RR-3), located between the upper and middle zones of the Royal River where the pseudo-UDM and CDM met, were selected from archived cores and were processed at the SAIC Environmental Testing Center (Figures 2-5 and 2-10). All cores consisted of black silty clay, but had an oxidized (gray) exterior. Because of the paucity of microfossils in many of the sediment samples from the upper reach of the Royal River during the original analysis, one of the additional cores (RR-3) was split into two sections to evaluate the effect of the preservative. For this discussion, the sample preserved in formalin is referred to as RR$3 F$, and the sample preserved in methanol is referred to as RR-3M.

Mineralogy. The coarse fraction from the top of the cores contained plant parts, wood chips, and shell fragments. Large bivalve mollusca fragments and an intact gastropod were found in Core RR-6. The fine fraction of the cores contained many of the trace components seen previously in the Royal River (Table 4-2; Figure 4-30). Samples RR-3M and RR-3F were identical in terms of mineralogical analysis, and so the data are shown only once on Figure 4-30. Quartz was predominant in all samples with micas common. Black porous material and plant fragments were common at RR-6 and RR-3, and very abundant in RR-5. Dark minerals, flyash, insect parts, and planktonic diatoms were rare. One textured ostracod was found in RR-6. The samples collected from the additional cores were similar in mineralogical composition to surrounding Cores RR-8, RR-15, and RR-26 and represent a combination of the upper and middle region means. Fibrous minerals and bryozoan fragments, indicators of the outer river region, were absent in all cores. Rare shell fragments in the additional cores were more typical of the upper and middle regions than their more common occurrence in the outer region.

Microfossils. The total number of individual microfossils was higher in the additional cores despite having been stored for over 18 months. Calcareous species (mudflat and shelf calcareous foraminifera, ostracods), however, were reduced in abundance relative to the original Royal River core analyses. This may be due to dissolution of the calcareous specimens (agglutinated species consist primarily of silica). Only Core RR- 6 had a significant number of both mudflat and shelf species, which potentially were protected from dissolution by the high concentration of calcium carbonate of large calcareous bivalve fragments embedded in the core. The data from the additional cores, therefore, probably do not represent the actual calcareous foraminifera or ostracod populations at these sites. 
A
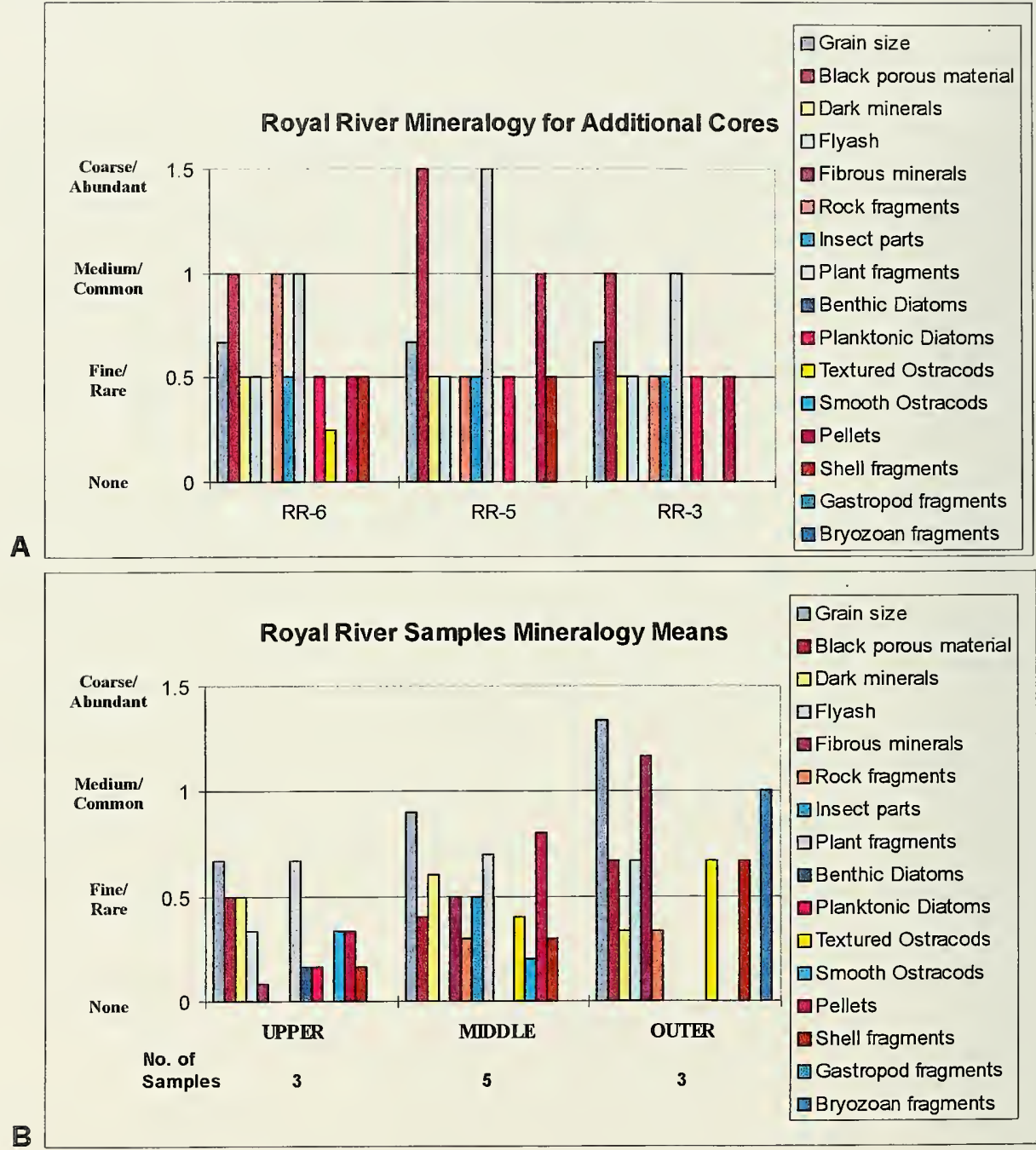

Figure 4-30. Histograms of abundance (rare, common, abundant) and grain size (fine, medium, coarse) for the mineralogical components of (A) the three additional Royal River cores, in comparison with the (B) mean mineralogical composition for the baseline Royal River cores 
In the additional Royal River cores, thecamoebians varied from $7 \%$ to $17 \%$ in the samples (Figure 4-31A). In composition, the microfossils in the cores were comparable to RR-26 located in the upper middle reach, with the exception of mudflat calcareous specimens. The percentage of thecamoebians in the additional cores was close to that in RR-26 and increased with the proximity of the core location to the upper river region.

Marsh foraminifera were predominant in all samples, however, many of them had poorly preserved shells. Mudflat calcareous foraminifera were absent in RR-3F and RR$3 \mathrm{M}$, and composed only a small percentage of RR-5 and RR-6. Shelf calcareous foraminifera were found in RR-6, and shelf agglutinated foraminifera varied from $0.6 \%$ to $4 \%$ in the four cores. One of the shelf agglutinated specimens in RR-6, Martinotiella communis, was not seen in any of the prior samples from the Royal River or at the PDS. The small percentage of shelf agglutinated foraminifera was greater than previously seen in the Royal River cores in which only one had been observed in RR-26. The shelf calcareous foraminifera also had a greater abundance in RR- 6 than seen before at RR-18 in the middle region.

Comparison of formalin and methanol solutions in samples from RR-3 was limited to the preservation of non-calcareous species due to the apparent dissolution of the calcareous fraction. Almost double the number of thecamoebians and a few more shelf agglutinated specimens were counted in the RR-3M (methanol) samples than found in the RR-3F (formalin) samples (Figure 4-31B). The microfossil content in the three additional cores had a greater density than in the Royal River cores processed in 1995 , which had been preserved in methanol, possibly reflecting the difference in preservation solution used. However, because the formalin and methanol preserved solutions were similar in the numbers per tray, the greater density probably was representative of a larger microorganism community in this transition area between the upper and middle regions.

The additional Royal River cores were classified with respect to the actual dredging plan as follows: RR-6, pseudo-UDM (phase 1); RR-5, pseudo-UDM (phase 2) near the border with CDM; and RR-3, CDM (Figure 2-10). The cores did not have distinct enough characteristics to classify them as separate regions, therefore, the later portion dredged for CDM shared some characteristics with the pseudo-UDM.

The microfossil abundance and total number (the density was not calculated for the additional cores) of the additional cores were comparable to the PDS grab and core samples. Although the mudflat calcareous specimens were reduced in the additional cores, the pseudo-UDM grab and core sample means were more consistent with Cores RR-6 and RR-5 than those previously described for the upper region. The additional cores provided evidence that some of the shelf species in the CDM, pseudo-UDM core, and pseudo-UDM grab samples were probably derived from the areas surrounding Cores RR-3, RR-5, and RR-6 in the Royal River. 

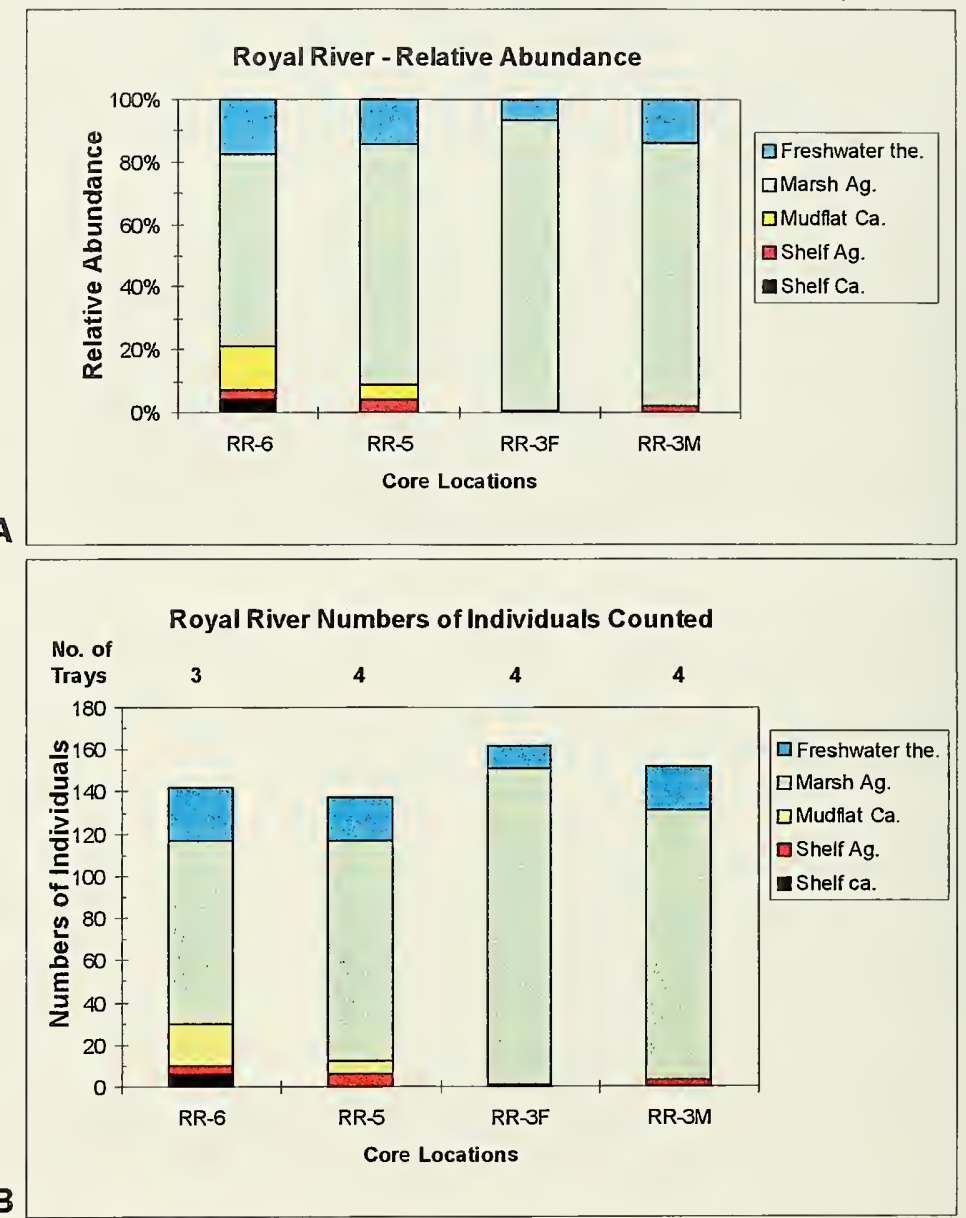

Figure 4-31. Histograms of relative abundance of microfossils and the numbers of individuals counted for the given number of trays analyzed for additional Royal River cores. Core RR-3 had similar abundances of microfossils preserved in formalin (F) and methanol (M) 


\subsection{Multivariate Statistical Analysis of Fine Fraction Results}

Multivariate statistics were used to evaluate whether there were statistically significant differences between the three layers (CDM, UDM, and AMB) classified from the postcap cores. As described in Section 3.10, several statistical methods were used, with the primary difference dependent on how the data were prepared. For clustering and MDS results (Section 4.7.1), the samples were analyzed prior to the layer classifications. Two statistical tests were conducted on the sample groups using the classifications derived after visual and microfossil results were compiled: an analysis of similarities test (Section 4.7.2), and an evaluation of discriminant statistics (Section 4.7.3). The analysis of similarities tested the null hypothesis that there was no difference between the UDM and CDM layers from the postcap cores. The discriminant statistics were then utilized to visually show the strength of the layer differences using both microfossil and mineralogical results.

\subsubsection{Clustering and Multi-Dimensional Scaling Results}

The fine fraction mineralogy and microfossil results were analyzed to determine the statistical similarity (cluster) and dissimilarity (MDS ordination) between samples, and to quantitatively evaluate the strength of any resultant clustering among the samples. More detailed information on the statistical methods is available in Section 3.10. The results are provided in a variety of graphical formats in the figures below. In order to better interpret the information, we first briefly describe the statistical output, and how it is presented.

The first analysis that we conducted, using the PRIMER clustering program (BrayCurtis similarity index), independently determined the similarities between sample data points based on multiple variables, and then grouped them accordingly to generate a similarity matrix. The matrix of samples was created with the similarities linked, with the links shown in a hierarchy and displayed on a dendrogram. The dendrograms provided below display the agglomerate clustering based on similarity. The higher the level of groupings on the dendrogram, the more similar those groups of samples were, relative to the mineralogy composition or the microfossil community structure.

The second test, a non-metric multi-dimensional scaling (MDS), provided an ordination, or map, of samples showing the inter-relationships between samples on a continuous scale. The ordination plots shown in the results below were constructed by an iterative procedure, which successively refined the positions of the samples to reflect the similarity relations between individual samples. The MDS method was used to produce a two-dimensional representation of the data from a three-dimensional dataset as shown in the ordination plot, and calculated a value called "stress" which provided an indication of the fit of the data in two-dimensional (2D) space. Stress values of up to 0.1 correspond to good to excellent 2D representation, values of up to 0.2 indicate the results are useful but 
should be used with additional statistical techniques, and stress values of up to 0.3 indicate that the values are almost arbitrarily located in the $2 \mathrm{D}$ ordination plot.

Following the creation of the cluster dendrograms and MDS ordination plots, individual samples were colored according to their original classification: AMB is red, pseudo-UDM is blue, and CDM is yellow. The results of both the cluster and MDS analyses are presented together in order to evaluate the overall ability to distinguish the three groups of samples.

Microfossils. Statistical analysis on the microfossil data showed that the AMB samples were most similar to each other, while there was overlap between the pseudoUDM and CDM samples (Figure 4-32). The dendrogram indicated that the AMB samples shared approximately $22 \%$ similarities with the dredged material samples. The ordination plot was consistent with the dendrogram, showing a distinct cluster for AMB samples, and less distinct clusters for CDM and UDM (Figure-4-32). The CDM and pseudo-UDM samples were distinguishable, but more closely associated to each other (sharing more similarities, therefore overlapping clustering on the ordination plot) than with the AMB samples. The CDM samples were clustered more compactly than the pseudo-UDM samples in microfossil analyses. The low stress value (0.12) of the MDS plot indicates that the two-dimensional depiction was an accurate representative of statistical groupings.

In order to further clarify the clusters on the ordination plot, the pseudo-UDM samples were divided into different shades of blue according to the clustering on the dendrogram. Samples that shared $68 \%$ or greater similarities were circled. All four pseudo-UDM samples circled with the CDM were located at the top of the pseudo-UDM interval in the cores. Samples 31 and 25 both contained a small pocket of sand that may have been from the disposal of CDM (Table 4-9). Sample 10, from the lower part of the B core, showed similarity to the AMB samples because it contained foraminifera found only on the continental shelf and most likely contained some ambient (or historical dredged) material.

Mineralogy. The dendrogram and ordination plot of the mineralogy results (Figure 4-33) showed a clear distinction between the ambient and dredged material samples, as in the microfossil results, but a more blurred grouping of CDM and pseudo-UDM samples. The ambient samples were isolated from the rest of the samples on the dendrogram. Two small groups of CDM samples and one group of five pseudo-UDM samples shared $68 \%$ similarity in mineralogy composition (Figure 4-33). Many CDM and pseudo-UDM samples were grouped together. The moderate-to-high stress level of the mineralogy ordination plot $(0.21)$ indicated that the $2 \mathrm{D}$ representation of the groups MDS plot was useful, but should be evaluated in light of alternate statistical tests. 
Table 4-9

Sample Identification for Statistical Analyses

\begin{tabular}{|c|c|c|c|}
\hline Sample No. & Core & Depth (cm) & Core Layer \\
\hline 1 & A-9 & $6-10$ & CDM \\
\hline 2 & A-9 & $16-20$ & $\mathrm{CDM}$ \\
\hline 3 & $\mathrm{~A}-9$ & $30-34$ & $\mathrm{CDM}$ \\
\hline 4 & A-9 & $39-43$ & UDM \\
\hline 5 & A-9 & $48-52$ & UDM \\
\hline 6 & A-9 & $53-57$ & UDM \\
\hline$\overline{7}$ & $\overline{\mathrm{B}-1}$ & $3-7$ & $\overline{\overline{C D D M}}$ \\
\hline 8 & B-1 & $10-14$ & $\mathrm{CDM}$ \\
\hline 9 & $\mathrm{~B}-1$ & $18-22$ & UDM \\
\hline 10 & B-1 & $24-31$ & UDM \\
\hline$\overline{111}$ & $\mathrm{C}-1$ & $0-4$ & $\overline{\overline{C D M}}$ \\
\hline 12 & $\mathrm{C}-1$ & $7-11$ & UDM \\
\hline 13 & $\mathrm{C}-1$ & $15-19$ & UDM \\
\hline 14 & $\mathrm{C}-1$ & $26-30$ & AMB \\
\hline & $\mathrm{C}-1$ & $40-44$ & AMB \\
\hline 16 & $\mathrm{D}-2$ & $2-6$ & $\mathrm{CDM}$ \\
\hline 17 & $\mathrm{D}-2$ & $8-12$ & CDM \\
\hline 18 & $\mathrm{D}-2$ & $19-23$ & UDM \\
\hline 19 & $\mathrm{D}-2$ & $25-29$ & UDM \\
\hline 20 & $\mathrm{D}-2$ & $34-37$ & UDM \\
\hline$\overline{21}$ & 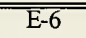 & $2-6$ & $\overline{\overline{C D M M}}$ \\
\hline 22 & E-6 & $8-12$ & CDM \\
\hline 23 & E-6 & $18-22$ & $\mathrm{CDM}$ \\
\hline 24 & E-6 & $30-34$ & $\mathrm{CDM}$ \\
\hline 25 & $\mathrm{E}-6$ & $40-44$ & UDM \\
\hline 26 & E-6 & $45-49$ & UDM \\
\hline 27 & E-6 & $50-54$ & UDM \\
\hline 28 & E-6 & $55-60$ & UDM \\
\hline 29 & E-6 & $65-69$ & UDM \\
\hline$\overline{30}$ & $\overline{\mathrm{G}-2}$ & $=4-8$ & CDM \\
\hline 31 & $\mathrm{G}-2$ & $10-13$ & UDM \\
\hline 32 & G-2 & $24-28$ & UDM \\
\hline 33 & $\mathrm{G}-2$ & $32-36$ & AMB \\
\hline 34 & $\mathrm{G}-2$ & $52-56$ & AMB \\
\hline$\overline{35}$ & $1-1$ & $0-4$ & CDM \\
\hline 36 & I-1 & $8-12$ & CDM \\
\hline 37 & $1-1$ & $15-19$ & $\mathrm{CDM}$ \\
\hline 38 & $\mathrm{I}-1$ & $22-26$ & UDM \\
\hline 39 & $\mathrm{I}-1$ & $27-28$ & UDM \\
\hline 40 & $\mathrm{I}-1$ & $40-44$ & UDM \\
\hline
\end{tabular}




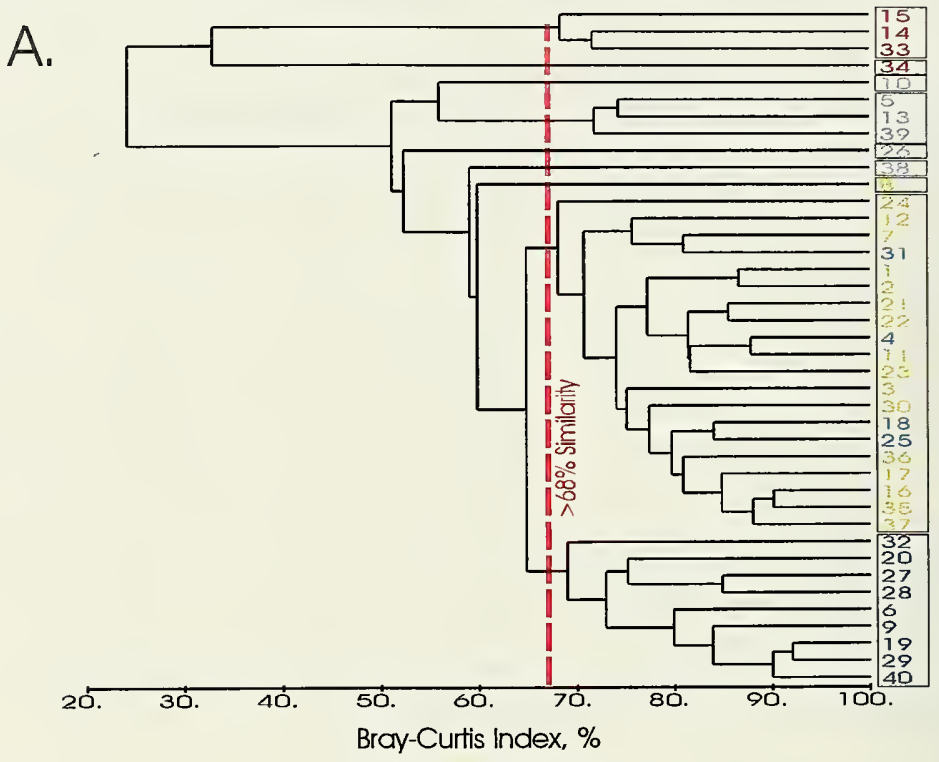

B.

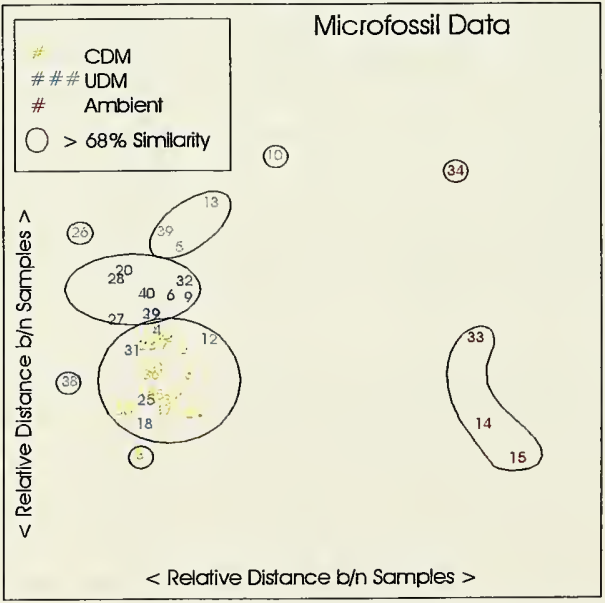

Figure 4-32. (A) Dendrogram of hierarchical agglomerate clustering using Bray-Curtis similarity index of microfossil data from PDS cores. (B) 2-D multidimensional scaling ordination plot (Stress $=0.12$ ) calculated from ranked similarity matrix of microfossil data. Sample numbers described in Table 4-9, see text for explanation of color scheme 


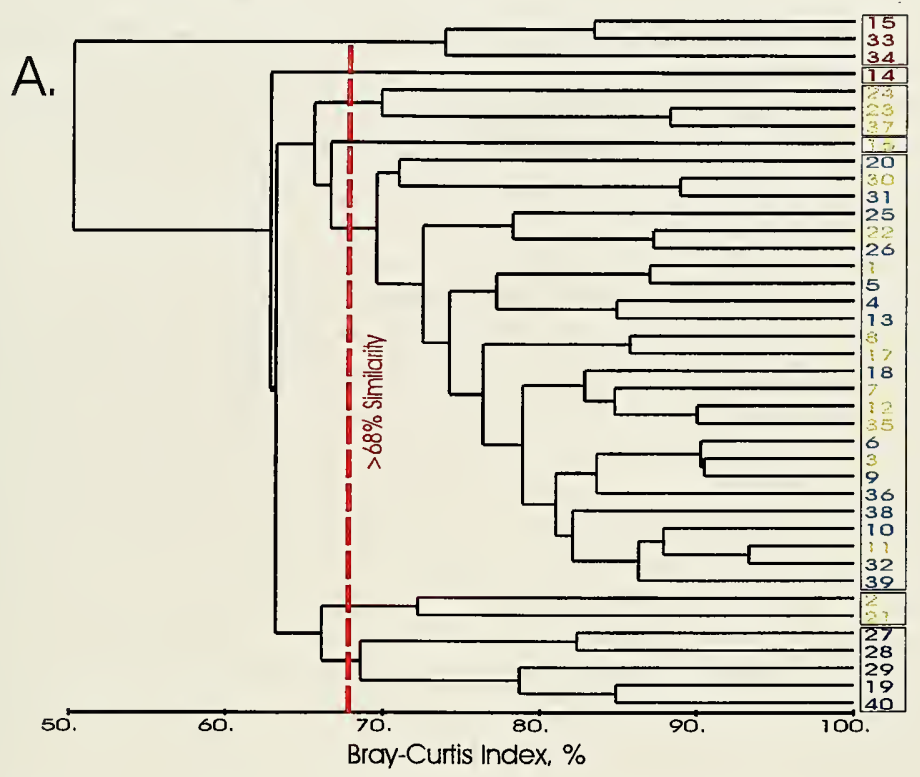

B.

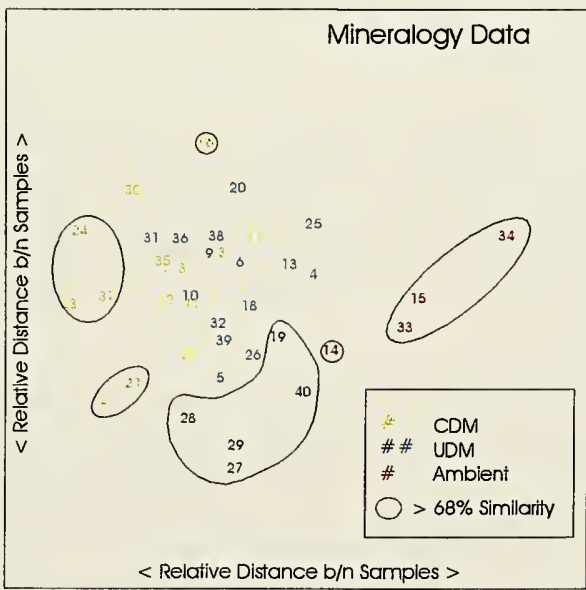

Figure 4-33. (A) Dendrogram of hierarchical agglomerate clustering using Bray-Curtis similarity index of mineralogy data from PDS cores. (B) 2-D multidimensional scaling ordination plot (Stress $=0.21$ ) calculated from ranked similarity matrix of mineralogy data. Sample numbers described in Table 4-9, see text for explanation of color scheme 
As with the microfossil plots, the pseudo-UDM samples were divided into different shades of blue according to the clustering on the dendrogram. Samples that shared $68 \%$ or greater similarities were circled. The MDS plot does show general groupings of the samples with some overlap between the CDM and pseudo-UDM samples.

\subsubsection{Analysis of Similarities}

After analyzing the microfossil and mineralogical results of the core samples and conducting clustering analysis, there was an overlap of characteristics between pseudoUDM and CDM. Therefore, a null hypothesis was tested to determine the statistical significance of the difference between CDM and pseudo-UDM samples. Again, the null hypothesis was that no differences existed between the CDM and pseudo-UDM samples.

An ANOSIM randomization test was conducted on microfossil data. ANOSIM is based on a non-parametric test analogous to standard parametric analysis of variance (ANOVA). For this test, the classification of the samples occurred prior to analysis and ambient samples were not included. The test compared the difference between the CDM and pseudo-UDM samples with the differences in the samples within each group displayed on the MDS plot. The program calculated a global R value of 0.297 . The null hypothesis, that no differences exist between the CDM and pseudo-UDM samples, was rejected with a significance level of $\mathrm{p}<0.001$.

\subsubsection{Discriminant Statistics}

Using SPSS ${ }^{\circledR}$ Professional Statistics 6.1, we performed a discriminant statistical analysis on the mineralogy and microfossil results from the core samples. Discriminant statistics is a multi-variable technique to measure the degree of association between groups of data. Because the groups were pre-determined based on the visual descriptions, the success of discriminant classification allowed an estimate of the actual differences or similarities between groups.

The microfossil data were grouped into five categories for this analysis: freshwater thecamoebians, marsh foraminifera, mudflat foraminifera, shelf agglutinated foraminifera, and shelf calcareous foraminifera. The relative abundance of the five groups of species were calculated for individual samples. Mineralogical parameter abundances were used as described above. Each sample was then grouped with the AMB, pseudo-UDM, or CDM classifications based on the visual core descriptions and on microfossil analyses.

Following separation into groups, the group means, standard deviation, and discriminant scores were calculated, and the scores were graphed according to two canonical discriminant functions. The canonical functions represented the ordination axes that best separated the pre-determined groups. The SPSS program then determined the 
percentage of samples appropriately classified into the pre-determined groups.

Discriminant statistical analyses of both the mineralogic composition and the microfossil assemblage classified $95 \%$ of the core samples correctly according to the designated layer. The graphs display three clusters of core samples which were described by two canonical discriminant functions (Figure 4-34). The first function presents the largest difference in the sample group based on the multi-variable composition, which clearly separated the AMB material from the disposed dredged material. The second function determines the next largest difference between the layers; the pseudo-UDM and CDM were more closely associated, though distinguishable. Similar to the MDS ordination plot, the microfossil discriminant scores showed denser clusters with greater distances between layer means than the mineralogy. The mean values for each layer (CDM, pseudo-UDM, ambient) was marked. The top samples of pseudo-UDM, from cores with more than one pseudo-UDM sample, are marked on the graph and tended to be close to the CDM cluster on both graphs. 


\section{Mineralogy Discriminant Scores}

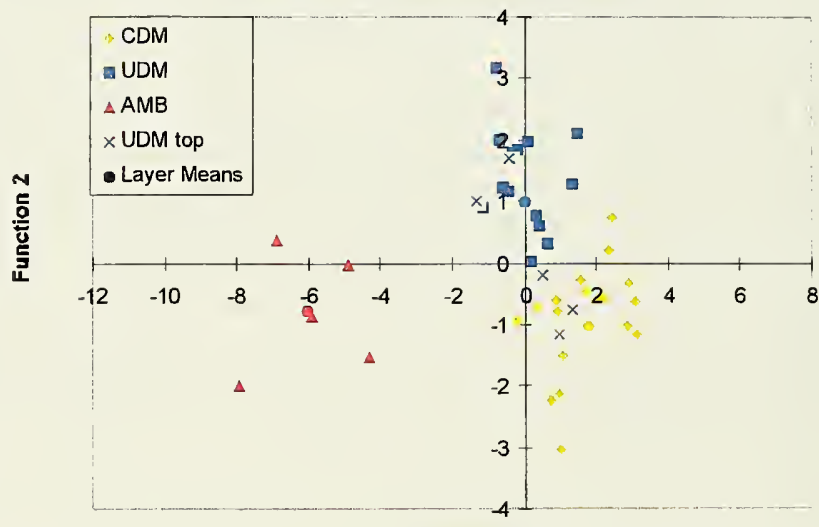

A

Function 1

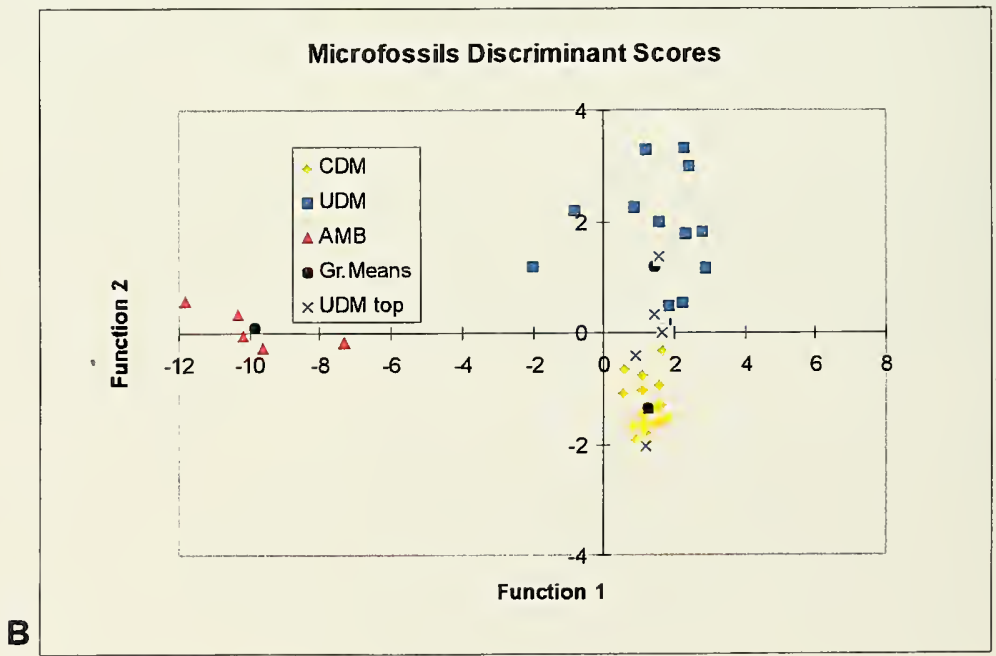

Figure 4-34. Plots of (A) mineralogy and (B) microfossil discriminant scores showing distinct clusters of samples according to layers in PDS cores 


\subsection{DISCUSSION}

\subsection{Assessment of the Footprint of the Capped Disposal Mound}

The demonstrated ability to form a discrete deposit of dredged material on the complex topography of the seafloor at the PDS was a key element to the success of the Royal River Project. Prior to the project, the DAMOS Capping Model was used to predict the size of the capped dredged material mound. The Capping Model is a tool to approximate the size of a mound formed from point dumping of material in a specified water depth. This model has proven useful in managing the deposition of dredged material at other disposal sites with subtle relief. This model does not include a correction for bottom topography. Therefore, the measured footprint of both the pseudo-UDM and CDM deposits on the floor of PDS was evaluated relative to the shape of the mound as predicted by the DAMOS Capping Model.

Based on the amount of dredged material disposed at the PDA buoy during the Royal River Capping Experiment $\left(39,500 \mathrm{~m}^{3}\right.$ pseudo-UDM and $\left.22,200 \mathrm{~m}^{3} \mathrm{CDM}\right)$, the DAMOS Capping Model predicted the formation of a conical pseudo-UDM deposit approximately $1.2 \mathrm{~m}$ high, with flanks extending up to $250 \mathrm{~m}$ from the central point of disposal, and a $20 \mathrm{~cm}$ thick cap. Although the capping volume was less than what is generally required in an actual capping project $(\geq 50 \mathrm{~cm})$, the model was still considered useful despite the small volumes used. These results were assessed in light of the measured deposit of pseudo-UDM (Section 5.1.1) and cap (Section 5.1.2) material.

\subsubsection{Pseudo-Unacceptably Contaminated Dredged Material (Pseudo-UDM)}

Typically in a capping project, sequential bathymetric surveys are used to determine the overall shape and height of a disposal mound $>20 \mathrm{~cm}$ thick (the resolution of the bathymetric method), and sediment-profile photographs are then used to map the apron of material of $<20 \mathrm{~cm}$. In the Royal River Project, bathymetry detected accumulation of dredged material in close proximity to the PDA buoy position following disposal of the pseudo-UDM. The bathymetric footprint consisted of two lobes, with an overall width of approximately $300 \mathrm{~m}$ (Figure 4-12). The footprint of the mound was concentrated within the naturally occurring basin feature detected in the southern quadrant of PDS.

Due to the complex bottom topography at PDS, however, there was a degree of uncertainty associated with the bathymetric results due to survey artifacts from replicate surveys over strong topographic features. The result is that the thickness of dredged material may have been overestimated in areas, especially along the eastern lobe which was located over a pre-existing topographic high (Figure 4-13). Because of the uncertainty of the bathymetric footprint, and the overall low height of the pseudo-UDM deposit, the 
REMOTS $^{\circledast}$ and core data were used to evaluate the overall footprint of the mound, rather than using these tools only to confirm the presence of the outlying apron.

The thickness of the pseudo-UDM deposit was mapped using REMOTS ${ }^{\circledR}$ information from the pseudo-UDM survey in November 1996, and the thicknesses of pseudo-UDM collected by the cores during the postcap survey. At many stations the REMOTS ${ }^{\circledR}$ data provided only a minimum thickness, as often the dredged material was thicker than the penetration of the REMOTS ${ }^{\circledR}$ camera. The core thicknesses should also be considered minimum, because the measurements were made after the placement of the cap so the material probably consolidated to some extent. In addition, only two cores actually penetrated into ambient, so that the pseudo-UDM collected in the postcap cores was estimated as minimum thickness (as displayed on Figure 5-1).

The maximum thickness of pseudo-UDM $\left(>20 \mathrm{~cm}\right.$ ) as measured by REMOTS ${ }^{\circledR}$ and cores was concentrated to the southeast of the buoy, consistent with bathymetric data. The core data (Core G2) indicated that the bathymetric footprint was indeed overestimated in the eastern lobe, as a $22 \mathrm{~cm}$ layer of pseudo-UDM was measured over ambient, rather than the 75 to $100 \mathrm{~cm}$ calculated by the depth difference method (Figure 5-1).

Within 100 meters of the disposal point, sediment-profile images and core data resulted in pseudo-UDM thicknesses of $10-20 \mathrm{~cm}$, often greater than camera penetration (maximum value $>17 \mathrm{~cm}$ ). The spread of dredged material was limited to within $200 \mathrm{~m}$ (upslope) towards the NW, but was present west, east, and south of the disposal point (Figure 5-1). The only exception was the station located $200 \mathrm{~m} \mathrm{SE}$ of the center, which was located on a rocky, high point as determined from the baseline REMOTS ${ }^{\circledR}$ survey. There was an apron of material of approximately $3 \mathrm{~cm}$ towards the south and east located at those stations measured $300 \mathrm{~m}$ away from the center, including $300 \mathrm{~S}$ and $300 \mathrm{SE}$ (downslope). No pseudo-UDM was measured at the stations located 400 meters away from the center.

These data suggested that the overall spread of material was relatively consistent with the DAMOS Capping Model. The model predicted a circular deposit around the center disposal point with a radius of $250 \mathrm{~m}$ (diameter $500 \mathrm{~m}$ ). Because of the slope of the basin towards the southeast, the material predictably spread further in that direction (between 300 and $400 \mathrm{~m}$ ) relative to the northwest $(<200 \mathrm{~m})$, so that the north-south axis of the deposit, according to the REMOTS ${ }^{\circledR}$ data, was approximately $500 \mathrm{~m}$ (Figure 5-1). In general, the deposit of pseudo-UDM appeared to be slightly smaller than predicted by the model, but because of the slope and the overall uneven bottom topography, the actual footprint was probably patchy and therefore difficult to reliably contour, especially with many values representing minimum thicknesses. 


\section{Portland Disposal Site Royal River Project Area Detection of Pseudo-UDM at Postcap Core and Precap REMOTS $®$ Survey Stations} over Precap Bathy metry

Disposal Site

Boundary

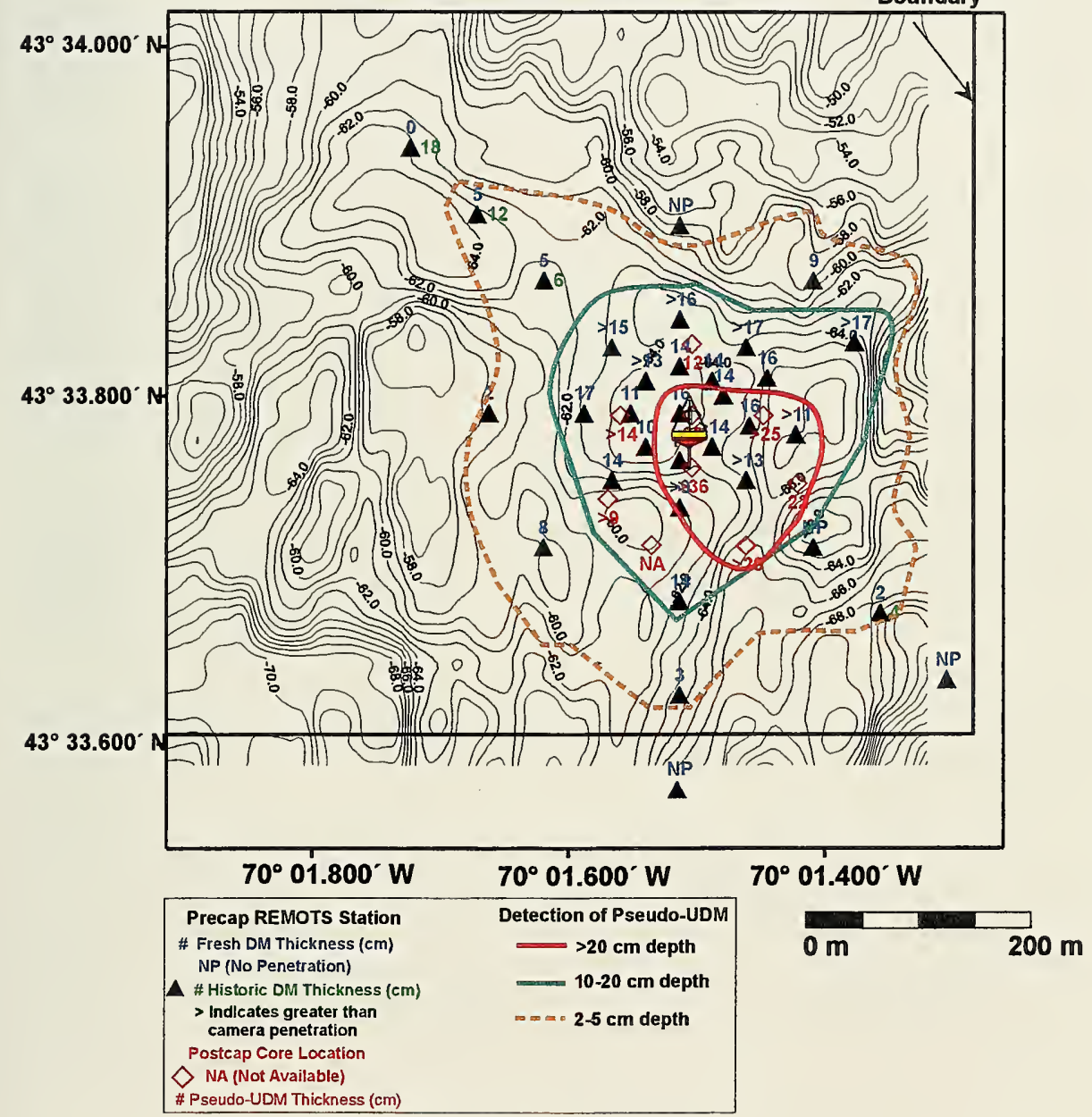

Figure 5-1. Thickness of pseudo-UDM measured by REMOTS $($ and core data overlaid on a postcap bathymetric chart (NAD 27), $1.0 \mathrm{~m}$ contour interval (MLLW) 


\section{Portland Disposal Site Royal River Project Area Detection of CDM at Postcap REMOTS ${ }^{8}$ Survey Stations and Core Locations over} Postcap Bathymetry Disposal Site

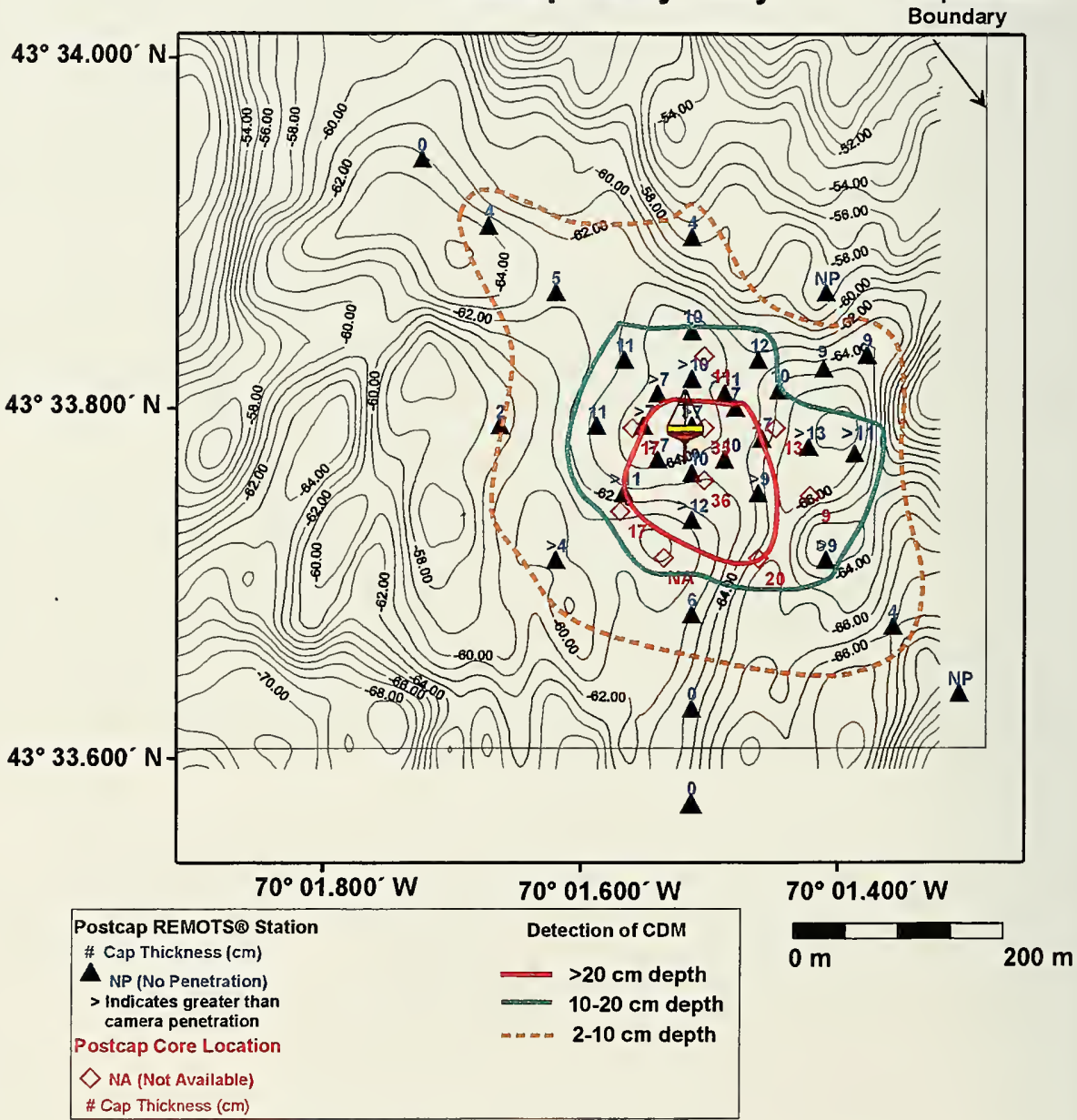

Figure 5-2. Thickness of CDM measured by REMOTS $®$ and core data overlaid on postcap bathymetric chart of (NAD 27), $1.0 \mathrm{~m}$ contour interval (MLLW) 


\subsubsection{Capping Dredged Material (CDM)}

Because of the small volume of CDM, survey artifacts associated with the bathymetric data caused a higher degree of uncertainty in mapping the CDM deposit. Therefore the CDM was mapped using sediment-profile images and core data from the postcap survey, so that a cap footprint could be estimated. Following analysis of the CDM deposit is a discussion of the total mound thickness relative to that predicted by the DAMOS Capping Model.

REMOTS ${ }^{\circledR}$ data were most useful in mapping the areal distribution of CDM at the project site, whereas core data often provided actual cap thicknesses at discrete locations. The greatest cap thickness, of $>20 \mathrm{~cm}$, occurred again southeast of the buoy (Figure 5-2). Core data showed the cap to range from a maximum of $35 \mathrm{~cm}$ near the center of the mound (CTR, 50S), to $20 \mathrm{~cm}$ within a circular area with a diameter of up to $200 \mathrm{~m}$. REMOTS $^{\circledR}$ data indicated that CDM was present from the thinnest layers $(1-2 \mathrm{~cm})$ at the peripheral stations located $300 \mathrm{~m}$ from the center, to full camera penetration at CTR. Therefore, cap material was present over almost all of the survey area, except that the material was not transported as far along the southern survey axis as the pseudo-UDM.

Total thickness of both pseudo-UDM and CDM, from core data, indicated a minimum of $60-70 \mathrm{~cm}$ in the center of the deposit. This value is a minimum because often the gravity cores did not penetrate into ambient. If we assume that core penetration and/or recovery was limited by the coarser ambient sediments, the thicknesses represent actual total deposit thicknesses.

The DAMOS Capping Model predicted a mound of material $1.2 \mathrm{~m}$ high, with a cap thickness of $20 \mathrm{~cm}$. The measured thickness of CDM was $>20 \mathrm{~cm}$ in many areas, but the overall mound appeared to have a lower relief than predicted. Compared to the predicted spread of material from the model, the Royal River mound was about the same areal dimensions, but the overall height was $<1 \mathrm{~m}$. The material thickness on the seafloor, however, was patchy so that discrete measurements may underestimate the overall thickness of the material. To reliably approximate the thicknesses of the disposal mound over the entire survey area in a region of strong seafloor topography, a much higher resolution acoustic survey (i.e., multibeam) would be necessary (Section 5.3). In terms of modeling a deposit on the seafloor of PDS, a more accurate prediction would require a model that accounts for bottom topography and slope. In general, however, the deposit was in good agreement with the model predictions.

\subsection{Tracking Dredged Material on the Seafloor}

A major goal of the Royal River Project was to select a tracer from the project area that would be distinct enough to reliably identify pseudo-UDM and CDM on the PDS 
seafloor. No single tracer was identified to reliably mark a specific reach of the Royal River, but several parameters had relatively limited ranges so that an approach of using multiple parameters was selected (Section 5.2.1). This approach showed statistical reliability, but there were some discrepancies that may be attributed to dredging and disposal operations (Section 5.2.2), as well as the presence of historical dredged material at the site (Section 5.2.3).

\subsubsection{Evaluation of Estuary Tracers}

Detailed analysis of selected Royal River cores (original and additional cores) indicated that two effective sets of tracers, microfossils and mineralogical components in the sediment, could be used to identify source material removed from the upper and outer reaches of the river. From the overall set of components, there were several mineralogical and biological components that were present in the upper/middle reaches and absent in the outer reach (insect parts, plant fragments, pellets), and one that was present everywhere except in the upper reach (fibrous minerals; Figure 5-3). Similarly, several biological components (diatoms, ostracods, and bryozoans are included in biological components for this discussion) were unique to a specific reach of the river. Diatoms (upper), smooth ostracods (upper), and bryozoan fragments (outer) had ranges that did not overlap into the middle reach, but the presence of these species were very rare. Thecamoebians were present primarily in the upper and middle reaches, but one specimen also was noted in one outer reach core (RR-12; Figure 5-3). Finally, the rare occurrence of shelf species in the Royal River increased the uncertainty of the use of this tracer for ambient sediments.

The Royal River data indicated two factors that were important in determining sediment tracers that could be used to distinguish the upper and outer reaches. The first was the coastal ecological zonations (Figure 2-7). Differences in species composition of the microorganism populations corresponded to the contrasts between the freshwater habitat of the upper river zone versus the brackish and saltwater environments of the middle and outer zones. The distribution of the biological components was consistent with these zonations, including the distribution of the diatoms, ostracods, and freshwater thecamoebians. The primary exception was the limited (1-2 individuals/core) observation of shelf agglutinated and calcareous foraminifera within the boundary between the upper and middle river reaches (RR-6, RR-26, RR-18). The presence of plant and insect fragments in the upper river reaches were also consistent with the upper river ecological zone.

The second factor important in distinguishing the upper and outer reaches of the estuary was the apparent relative hydrodynamic energy. The coarser grain size and dominance of the fibrous minerals in the outer reach were indicative that the fine-grained fraction was selectively removed. Because of tidal flushing within the river, identifying localized constituents for tracers was problematic. Environmental tracers are useful only if they are accurate indicators of the local, resident community and tend not to be transported 


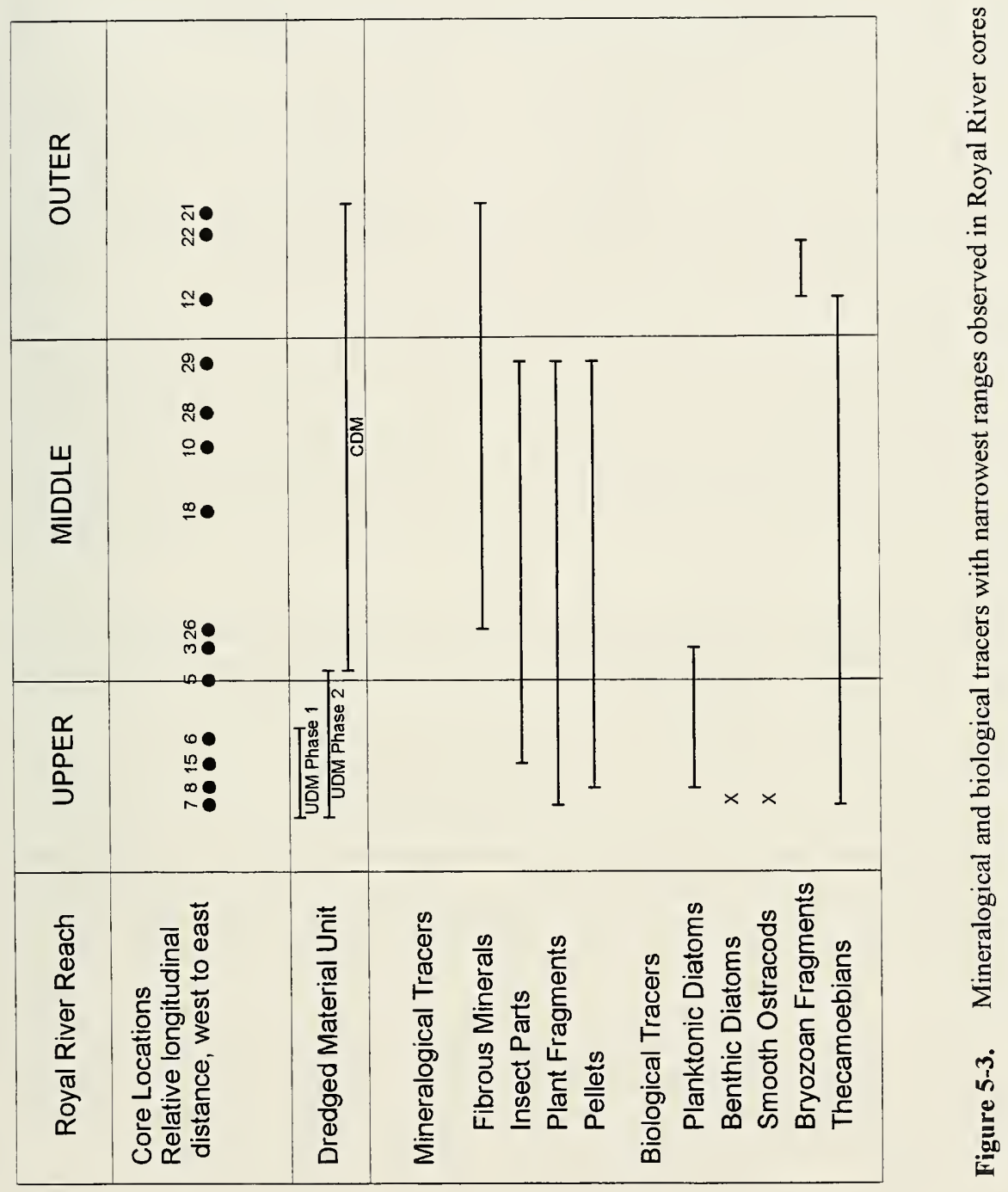


to or from other regions. Because thecamoebians and mudflat/marsh foraminifera are all benthic species that live on or in the substrate, transportation of the microfossils was likely limited. The state of naturally preserved foraminiferal tests and ostracods suggested that many individuals were collected near their habitat range.

Because no single tracer was found that was both 1) unique to the upper or outer reach of the Royal River, and 2) common throughout all of the cores collected in that particular reach, the method of combining data from several parameters to characterize each river zone was found to be the most practical. In this way, a particular sample was classified based on statistical groupings of a variety of parameters.

\subsubsection{Statistical Strength of Tracer Grouping Method}

The statistical methods used to group the samples collected over the Royal River Survey Area at PDS bore out the conclusion that the CDM and pseudo-UDM had overlapping characteristics, primarily due to the ranges of the tracers within the Royal River. In this section, a summary of the statistical conclusions are provided, then a discussion of the discrepancies that were brought out after the statistical analysis. These statistical discrepancies were found to be primarily related to the dredging and disposal operations.

All of the statistical tests that were conducted on the grab and core samples collected at the Royal River Project Area indicated that the ambient samples were most similar to each other, while there was overlap between the pseudo-UDM and CDM samples. The microfossil dendrogram indicated that the ambient samples shared approximately $22 \%$ similarities with the dredged material samples. The CDM grouping was more distinct than the UDM. In general, statistical analyses showed that tracers can be used to identify disposed dredged material layers removed from different regions of an estuary. However, they are more difficult to distinguish if the material was dredged from nearby regions (i.e., upper and middle) as opposed to separate regions (upper and outer). Also, the tracers were not found to be equally effective in distinguishing material origin. Overall, the biological indicators were statistically more robust than the mineralogical indicators.

Another major result, replicated by the ordination and discriminant statistical methods, was that four of the pseudo-UDM samples that were most similar to the CDM samples were located at the top of the pseudo-UDM interval in the cores. Because of the phased dredging, the last of the cap material was dredged from the same area as the material from pseudo-UDM Phase 2 (Figure 5-3). The overlap of the CDM and pseudoUDM groupings, therefore, was consistent with the disposal operations data. In addition, all of the core and grab samples had a lower relative abundance of freshwater thecamoebians and diatoms than the upper region of the Royal River. Thecamoebians and 
diatoms did increase in abundance with depth in the pseudo-UDM cores. It is likely that the pseudo-UDM grab and upper core samples were representative of material dredged during the second phase of UDM that extended into the upper middle reach. Finally, many of the CDM samples had components that were identified as only being in the upper and middle reaches, including insect parts, pellets, and an average of $4 \%$ thecamoebians. Again, this material was derived from the upper middle part of the River during the final phases of the project.

The presence of bryozoan fragments in the pseudo-UDM of two cores provided evidence for intermixing of CDM, as the range of bryozoans was limited to the outer reach. No bryozoan fragments were noted in the pseudo-UDM grab samples, but rare fragments were observed above and below the CDM/pseudo-UDM interface in Core E. The possible mechanisms for this include: mixing during the core retrieval and sampling process; disturbance of pseudo-UDM during CDM disposal; or possible sampling artifact (pseudo-UDM sample was collected at the interface). Mixing during core retrieval was likely for Core B, only half of which was sampled because the other half was waterlogged and disturbed. Even though the CDM and pseudo-UDM layers were clear in the half sampled, the presence of shelf foraminifera throughout was likely the result of mixing due to core retrieval.

Other discrepancies of unit classifications were considered to be the result of the presence of historical dredged material. For example, the presence of shelf species in the pseudo-UDM or freshwater thecamoebians in the ambient layer was investigated (Section 5.2.3).

\subsubsection{Differentiating Ambient from Historic Dredged Material}

The definition of the ambient sediment at the Royal River Survey Area must be clarified prior to a discussion of the samples recovered from this material. For our purposes, ambient sediment was defined as the native sediments of offshore Maine. There was an additional component of material present at the survey area prior to the initiation of the Royal River project. This included dredged material from the Harraseeket River project, and historical dredged material present prior to the baseline survey. The baseline REMOTS $^{\circledR}$ survey, conducted after the Harraseeket River project but prior to the Royal River project, indicated that dredged material was present in the survey area, most prevalently towards the northwest (Figure 4-7). The presence of dredged material in the project area hampered the interpretation of the ambient and pseudo-UDM interval of the cores to some extent. Ambient sediment and historical dredged material share some biological characteristics as a result of recolonization of benthic species, while both historical and recent dredged material will have a fresh or brackish water species component. 
The sediment composition and microfossil content of the ambient material prior to disposal of Royal River sediment was not studied. An overall description of the material, however, was reliably obtained from the samples collected in the postcap cores. In the ambient samples, shelf foraminifera composed about $83 \%$ of the relative abundance in the ambient layer samples. The ambient layer also had a high abundance of planktonic diatoms, pellets, dark minerals, and rock fragments.

Only two cores recovered sediment that was given a final characterization as ambient material ( $\mathrm{C}$ and $\mathrm{G}$ ). Both of these core sections had a small component of mudflat and marsh foraminifera. These brackish and freshwater microfossils in the ambient sediment suggested the presence of some historic dredged material. The marsh foraminifera, sorted sand, and the strong sewer smell in the ambient samples of Core C also provided indications of historical dredged material deposition.

Another sample possibly encountering historical dredged material was the deepest sample in Core A, collected approximately $55 \mathrm{~cm}$ from the top of the core. This sample contained over $20 \%$ thecamoebians and no shelf foraminifera characteristic of ambient sediments in PDS. Because the sample did not have the typical texture of dredged material, the sample was originally classified ambient during core descriptions; following microfossil analysis the sample was reclassified as pseudo-UDM. The ambient appearance of this material and the presence of shelf foraminifera in the 48 to $52 \mathrm{~cm}$ sample, however, suggested that the sample may actually be historical dredged material, rather than dredged material associated with the Royal River project.

Several of the grab and core samples classified as pseudo-UDM indicated the presence of shelf foraminifera. Although three Royal River cores (RR-26 and RR-18 from the 1995 analyses; as well as RR-6 from the summer 1997 analyses) indicated the rare presence of shelf foraminifera, the shelf foraminifera in the pseudo-UDM were relatively more abundant. It is likely that rapid recolonization of shelf species occurred after disposal events. Previous recolonization studies suggest that benthic calcareous foraminifera repopulate dredged material deposits soon after disposal, and increase in abundance with time (Rhoads et al. 1977).

A few of the calcareous species identified at the PDS were planktonic and live floating in the water. Planktonic species may descend through the pelagic environment and settle on the sediment surface or be captured and transported with disposed dredged material. Most foraminifera detected in the sediment samples were benthic species suggesting that recolonization was more common than surface contamination. The prevalence of planktonic diatoms in most core and grab samples as well as the top samples of the ambient layers, yet absence in Royal River samples, also indicated surface contamination by planktonic species in the dredged material at the disposal site. 


\subsection{Evaluation of Tools}

\subsubsection{Bathymetry}

Rather than providing a first order footprint of the dredged material deposit, as is traditional with DAMOS monitoring, single-beam bathymetry in the Royal River study provided only supplementary data due to the limitations over the complex bottom topography. The REMOTS ${ }^{\circledR}$ sediment-profile images and core data were useful in identifying some apparent depth changes as survey artifacts. In general, small volumes of sediment are difficult to track with the use of single-beam bathymetry, and even more problematic in an area like the PDS. The location of the mound over the relatively flat Royal River Survey Area provided more confidence in the data, and a thicker dredged material deposit would also strengthen the use of the single-beam tool. Changes in depth of $<0.25 \mathrm{~m}$ are generally undetectable due to the accumulation of errors originating from the motion of the survey vessel, and the various correctors applied to the raw data. As discussed in our recommendations (Section 7.0), the accuracy of mapping dredged material deposits on a topographically complex area such as the PDS would be greatly enhanced using multibeam bathymetry.

\subsubsection{Photography: Planview and REMOTS ${ }^{\circledR}$ Sediment-Profile Imaging}

The DAMOS Program has used REMOTS ${ }^{\circledR}$ sediment-profile photography for many years as a method of detecting the distribution of dredged material, as well as mapping benthic disturbance gradients and monitoring infaunal recolonization status. During the capping demonstration project, the sediment-profile camera was more instrumental than usual because of the limitations of the single-beam bathymetric data. The images collected by the REMOTS ${ }^{\circledast}$ camera demonstrated its ability to remotely visualize, differentiate, and map thin layers of sediment deposited on the seafloor that would otherwise be undetectable. The REMOTS ${ }^{\circledR}$ camera also was able to document the presence of historic dredged material originating from disposal operations at the DG buoy from 1990 to 1991 as well as the Harraseeket River material deposited in the fall of 1995. In future projects, the sedimentprofile images may prove to be useful in distinguishing project from historical dredged material, although ideally the presence of historical material will be limited.

\subsubsection{Grab Sampling and Coring}

Grab sampling and coring provided a cost-effective means to characterize the pseudo-UDM sediment, and to confirm the presence of pseudo-UDM in the postcap cores. The coring survey was also successful in documenting the placement of two distinct layers of dredged material over ambient sediments. One problem with the cores was the potential for core-induced mixing of the different layers. Storing the cores vertically prior to 
processing, and avoiding sampling from the outside of the core, would help to limit crosscontamination. Finally, longer cores into ambient material would provide more robust groundtruth data on the actual thickness of dredged material layers.

\subsubsection{Fine Fraction Tracer Technique}

Microfossils. Microfossil analysis proved to be a valuable tool to characterize the sediment composition of the Royal River and trace the dredged material at the PDS. The predominance or absence of shelf species in a sample clearly indicated whether the material was ambient or part of the pseudo-UDM, although the presence of historical dredged material at the site contributed some uncertainty. For a few samples, the classification of pseudo-UDM or ambient layers based on visual observations were re-categorized following the identification of microfossils. The percentage of thecamoebians was important in distinguishing the CDM from the pseudo-UDM. The density distribution of microfossils was also useful in identifying the CDM/pseudo-UDM horizons in the cores. The ratios of agglutinated to calcareous foraminifera and, more specifically, marsh to mudflat specimens, were not effective indicators because the ratios were inconsistent throughout the Royal River regions. Overall, limiting the fine fraction analysis to those components which are most representative of a narrow range in the dredging area (Figure 5-3) would increase the statistical reliability of sample identification using tracers.

Mineralogy. Overall, mineralogical observations provided a useful supplementary tool to characterize the river regions, dredged material, and ambient substrate. Descriptions of sediment mineralogy on a microscopic level were important for documenting indicators of river regions and using them to trace dredged material. Grain size analysis of the fine fraction alone was not sufficient to distinguish the pseudo-UDM layer from the ambient substrate, both of which had a medium grain size. Because all indicators were mostly rare components of the samples and may have patchy distributions, there is a degree of uncertainty in determining how representative they are of the entire area dredged. In addition, quantification of the data was difficult and not as precise as counting the individual species of microfossils. Duplicate analyses, however, increased the precision of the mineralogy analysis (the two splits of Core RR-3 contained identical mineralogical characteristics). 


\subsection{CONCLUSIONS}

- A discrete, capped dredged material mound was created and detected on the seafloor of Portland Disposal Site in the Royal River Project Area. Analysis of REMOTS ${ }^{\circledR}$ sediment-profile images, cores, and environmental tracers detected a discernible difference between the CDM, pseudo-UDM, and ambient material.

- An accumulation of pseudo-UDM was detected to the south and southeast of the PDA buoy position using single-beam bathymetry and REMOTS ${ }^{\circledR}$ sedimentprofile images, most reliably in the relatively flat-bottomed basin targeted for disposal.

- Accurately detecting material in the surrounding area of more complex topography by single-beam bathymetry alone was complicated by survey artifacts. A more accurate measurement of the thickness and footprint of the disposal mound could be obtained using higher resolution acoustic methods (i.e., multibeam).

- The relatively small volume of CDM placed over the initial pseudo-UDM deposit was measured and mapped with the use of sediment-profile photography and cores; minimum detected CDM thicknesses ranged from 10 to $35 \mathrm{~cm}$.

- The areal distribution of both pseudo-UDM and CDM, measured using sediment-profile photography and cores, was relatively consistent with the DAMOS Capping Model. Because of the slope and uneven bottom topography of the area surrounding the primary depositional basin, the material thickness appeared patchy and therefore difficult to reliably contour using only bathymetric methods.

- The Royal River data indicated two factors that were important in determining sediment tracers that could be used to distinguish the upper and outer reaches: the distribution of the biological components consistent with coastal zonations, and the relative hydrodynamic energy of the river reach.

- No single tracer was found that was both unique to one reach of the river, and commonly observed in all collected samples, so the method of combining several parameters was found to be most promising at classifying the material types. Limiting the fine fraction analysis to components that have the narrowest range in the dredging area would serve to both decrease the effort required to analyze the samples, as well as increase the statistical reliability of sample identification using tracers. 
- The fine fraction of the sediment had the most promising set of tracers. Overall, the biological indicators were statistically more robust than the mineralogical indicators.

- The statistical analyses showed that tracers successfully identified disposed dredged material layers taken from different regions of an estuary, but material from the middle reach had many overlapping characteristics that complicated the interpretation.

- The overlap of the pseudo-UDM and CDM samples collected in cores and grabs from the disposal mound in statistical analysis was consistent with the sequence of disposal operations.

- Because historical dredged material shares biological characteristics with both native, ambient sediment (recolonization by benthic species, settling of planktonic species), and with recent dredged material (presence of freshwater species), it theoretically can be identified. Limiting the presence of historical dredged material in a project area, however, would decrease the possibility of inaccurate identification.

- The tools used for the Royal River project each proved useful for different types of analysis; single-beam bathymetry was noted to be the most problematic due to the complex topography of the survey area.

- Overall, the Portland Disposal Site Capping Demonstration Project demonstrated that dredged material can be effectively placed, capped, and monitored at a deep water site. 


\subsection{RECOMMENDATIONS}

\subsection{Bathymetric Data Collection}

For areas of complex bottom topography (i.e., PDS, RDS, and CADS), a higher resolution single-beam bathymetric survey grid ( $5 \mathrm{~m}$ to $10 \mathrm{~m}$ lane spacing) is needed to reduce the effects of gridding (averaging) within a large cell, and in turn, minimize the appearance of survey artifacts. This would require the collection of more soundings within smaller cells, reducing errors attributed to averaging, but doubling or tripling the time required to conduct the survey and process the data.

For comprehensive, high resolution surveys, multibeam bathymetry systems have the capacity to collect the necessary volume of data to provide depth difference comparisons with significantly reduced error within a limited survey time. Shallow water $(<100 \mathrm{~m})$ multibeam systems emit up to 100 beams from a single transducer array to provide complete coverage (100 to $200 \%$ ) of the seafloor within a survey area. Survey lanes are usually spaced $50 \mathrm{~m}$ to $100 \mathrm{~m}$ apart reducing survey time relative to single-beam bathymetry. In addition, due to the $100 \%$ bottom coverage, multibeam data can be gridded into cells as small as $1 \mathrm{~m}^{2}$.

\subsection{Operational Control}

Dredging and disposal operations before and during the Portland Disposal Site Capping Demonstration Project contributed several variables that added to the level of uncertainty in data interpretation:

- Dredged material was already present in the area, both from historical disposal towards the northwest of the Royal River Project Area, and from the Harraseeket project;

- The upper part of the CDM layer (last cap material placed) was essentially the same material as the upper part of the UDM (last upper river material placed), causing discrepancies in statistical analyses of core samples;

- The relatively small volumes of both UDM and CDM made bathymetric methods more subject to error, as well as increasing the uncertainty of identifying thinner layers of sediment using tracer analysis.

For the Royal River project, an area of PDS that was least impacted by historical dredged material was selected prior to the initiation of the project. For future projects, this will be the most practical approach, although among the disposal sites monitored by DAMOS, finding an area completely free of historical dredged material is problematic. Had the Harraseeket River material not been disposed at the site, the complication by the presence of dredged material would have been restricted to the much older historical 
dredged material. The surface layers of this material, as discussed above, should have characteristics much more similar to the ambient sediment (i.e., abundant shelf and planktonic microorganism species), and so therefore would prove to be less of a complication.

The disposal plan used for the demonstration project was to use the suitable material from the outer reaches of an estuary to cap the sediments from the inner reach. Using this plan for other projects will result in material from the transitional area that also must be dredged. For a project with larger volumes, the core sampling scheme can be concentrated in narrow ranges of the core that represent the transition from pseudo-UDM to CDM, so that sampling of the middle reach material (presumably at the top of the mound) would be minimized. In addition, if the project volumes are large enough, perhaps the material dredged from transitional reaches, if suitable, could be placed at an alternate disposal location.

\subsection{Sediment Tracer Technique}

Several recommendations were derived from the sediment tracer and statistical analyses:

- Limit the tracer analysis to the individual components that have the narrowest range in the dredging area in order to decrease the level of effort required to analyze the samples, as well as increase the statistical reliability of sample identification. In hindsight, this analysis could be conducted on the Royal River project data, but for purposes of the demonstration project, a thorough analysis of all components was necessary for evaluation of the tracer technique.

- For future projects, efforts should focus on the microfossil analysis. Mineralogy observations should serve as a supplemental tool. The optimum tracer will be, however, site-specific.

- For a sufficiently large volume project, focus the sample and statistical analyses on sediment sampled from above and below the ambient/UDM and UDM/CDM boundaries. These boundaries can be preliminarily identified using abundances of shelf and freshwater species, then a detailed analysis of samples near the boundary can be conducted.

- Sample the ambient material and conduct a tracer analysis prior to initiation of the dredging project.

- Avoid areas with historical dredged material. If this is impossible and historical dredged material is present in the selected disposal area, sample and analyze the 
material for tracers prior to initiation of the dredging project as well as the native ambient sediments from a reference area.

- Formalin is recommended to be used as a preservative for microfossils instead of methanol to avoid dissolving calcareous tests.

- Multivariate statistical analysis proved to be a valuable tool to identify sample grouping patterns. Clustering (ordination) and MDS methods are most strongly recommended because the samples are analyzed with no bias from layer classifications from core descriptions.

- For more robust statistics, more consideration should be given to the absolute abundance of microfossils in the samples, especially for rare species. In samples where species are common, the abundance values are reliable to $\pm 5 \%$. The abundance of rare specimen that occur below $5 \%$ relative abundance has a greater range of uncertainty. If documentation of the abundance of rare specimen is important (e.g., shelf foraminifera in the Royal River), analyzing more sample trays would increase the accuracy of the results for rare species, but would also increase the time it takes to examine each sample. When the density of microfossils is very low, other techniques might be considered to examine a larger quantity of material, such as separation with heavy liquids. 


\subsection{REFERENCES}

Attanas, L.; Hinkley, M.J. 1997. A concise history of Yarmouth, ME. Yarmouth Historical Society, Yarmouth, ME.

Belknap, D.F., R.C. Shipp, R. Stuckenrat, J.T. Kelley, and H.W. Borns, Jr. 1989. Holocene sea-level change in coastal Maine. IN: Walter A. Anderson and Harold W. Borns, Jr. (Eds.), Neotectonics of Maine, Studies in Seismicity, Crustal Warping, and Sea-Level Change. Maine Geological Survey, Dept. of Conservation, August, ME.

Clarke, K.R.; Warwick, R.M., 1994. Change in marine communities: an approach to statistical analysis and interpretation. Plymouth: Plymouth Marine Laboratory, Natural Environmental Research Council, UK. 144pp.

Custom Communications (Custom) 1995. The state of Maine's port of Portland marine directory. City of Portland, Portland, ME.

Dolin, E. J.; Pederson, J. 1991. Marine-dredged materials management in Massachusetts: issues, options and the future. A student project of the MIT Sea Grant College Program with supervision and assistance from the Massachusetts Coastal Zone Management Office. MITSG 91-25. MCZM-TR-91-01.

Fredette, T.J. 1994. Disposal site capping management: New Haven Harbor. Reprinted from Dredging '94, Proceedings of the Second International Conference, November 13-16, 1994. U.S. Army Corps of Engineers, New England Division, Waltham, MA.

Maine State Planning Office (MSPO). 1983. The Geology of Maine's Coastline. Executive Department, MSPO, Maine.

McDowell, S.C.; Pace S.D. 1997. Oceanographic measurements at the Portland Disposal Site during the spring of 1996. SAIC Report No. 388. Final report submitted to the U.S. Army Corps of Engineers, New England Division, Waltham, MA.

Morris, J.T; Tufts, G.J. 1997. Monitoring cruise at the Central Long Island Sound Disposal Site, July 1994. SAIC Report No. 327. Final report submitted to the U.S. Army Corps of Engineers, New England Division, Waltham, MA.

Morris, J.T. 1996. DAMOS site management plans. SAIC Report No. 365. Final report submitted to the U.S. Army Corps of Engineers, New England Division, Waltham, MA. 
Morris, J.T.; Charles, J.; Inglin, D.C. 1996. Monitoring surveys of the New Haven Capping Project, 1993-1994. DAMOS Contribution 111. U.S. Army Corps of Engineers, New England Division, Waltham, MA.

Murray, P. M.; Selvitelli, P. 1996. DAMOS navigation and bathymetry standard operating procedures. SAIC Report No. 290. DAMOS reference report. U.S. Army Corps of Engineers, New England Division, Waltham, MA.

Nousis, M.J., 1994. SPSS Professional Statistics ${ }^{\mathrm{TM}}$ 6.1 Manual. SPSS Inc., Chicago, IL.

Rhoads, D.C.; Aller, R.C; Goldhaber, M.B. 1977. The influence of colonizing benthos on physical properties and chemical diagenesis of the estuarine seafloor. Ecology of Marine Benthos (B.C. Coull, ed). University of South Carolina Press, Columbia, SC.

SAIC. 1995. Sediment capping of subaqueous dredged material disposal mounds: An overview of the New England experience. DAMOS Contribution No. 95. U.S. Army Corps of Engineers, New England Division, Waltham, MA.

Wentworth, C.K. 1922. A scale grade and class terms of clastic sediments. J. Geo. 30:377-390.

Wiley, M.B. 1995. Deep water capping. DAMOS Contribution 98. U.S. Army Corps of Engineers, New England Division, Waltham, MA.

Wiley, M.B. 1996. Monitoring cruise at the Portland Disposal Site, July 1992. DAMOS Contribution 108. U.S. Army Corps of Engineers, New England Division, Waltham, MA. 



\section{INDEX}

barge, $2,7,19,24,69,74,87$

disposal, xiii, 5

benthos, xiv, $25,34,54,58,74,84,97$,

$102,126,127,128,129,132$

bivalve, 107

lobster, 67

mussels, 52, 67

bioturbation

excavation, xiv, 19, 69

buoy, $19,23,24,35,40,42,59,62,67$,

$69,87,89,119,120,123,129,131$

disposal, xiv, 7, 19, 23, 25, 34

capping, xiii, xiv, $2,3,5,7,16,19,23$, $24,28,34,42,48,51,53,59,87,89$, $119,120,123,129,131,132,133$

Central Long Island Sound (CLIS)

STNH-N, 3

STNH-S, 3

conductivity, 32

consolidation, 29

containment, xiii, $3,7,19,59$

contaminant, 2,3

CTD meter, 32

currents

speed, 11

density, 32, 45, 47, 84, 101, 102, 105,

$109,130,135$

deposition, xiv, $3,7,11,14,16,23,35$,

$59,62,69,87,89,101,119,128,131$

detritus, 52

disposal site

Boston Foul Ground (BFG), 5

Cape Arundel (CADS), 3, 5, 25, 133

Central Long Island Sound (CLIS), 3, 5

Massachusetts Bay (MBDS), 3, 5

Portland (PDS), xiii, xiv, 1, 3, 5, 7, $14,16,19,23,24,25,28,29,32$, $34,35,42,44,46,47,48,51,53$, $55,59,62,67,69,87,89,101$, $105,109,119,123,126,128,129$, $130,131,132,133$
Rockland (RDS), 3, 5, 23, 25, 133

erosion, 2, 29

fecal pellets, 54

fish, 14

finfish, 14

grain size, 40, 44, 46, 49, 54, 74, 84, 97 ,

$101,124,130$

habitat, xiv, 19, 59, 124, 126

National Oceanic and Atmospheric

Administration (NOAA), 32

nutrients

ammonia, 87

organics

polychlorinated biphenyl (PCB), 2

oxidation, 59

recolonization, xiv, $84,97,105,127$,

$128,129,132$

reference area, 135

REMOTS $^{\circledR}$, xiv, 23, 24, 28, 29, 34, 35,

$59,62,67,69,74,80,87,89,90,97$,

$120,123,127,129,131$

redox potential discontinuity (RPD), 62 resuspension, 25

RPD

REMOTS ${ }^{\circledR}$, redox potential

discontinuity (RPD), 62, 74, 89, 97

sediment

clay, $2,7,14,46,52,54,62,74,84$, 101,107

cobble, 67

gravel, 2, 46, 52, 53

resuspension, 25

sand, $2,7,14,24,46,47,51,52,53$, $54,55,67,84,87,89,97,101$, 112,128

silt, $2,7,11,14,46,47,52,54,59$, $62,67,74,84,89,101,107$

sediment sampling

cores, v, xiv, 16, 24, 29, 42, 43, 44, $45,46,47,48,49,50,51,52,53$,

$54,55,58,84,97,101,102,103$, 
$105,107,109,111,112,116,117$,

$120,123,124,126,127,128,129$,

$130,131,132,133,134,135$

grabs, xiv, xv, 23, 28, 40, 45, 46, 47,

$55,69,74,83,84,97,101,102$,

$105,109,126,127,128,129,132$

vibracores, xiv, 16, 42

shore station, 28

side-scan sonar, xiv, 23, 28, 29, 34, 59, $62,67,69$

species

dominance, $49,52,84,101,124$

statistical testing, xiv, 47, 48, 49, 50, 51, $101,105,111,112,113,116,117$, $124,126,130,131,132,133,134$, 135

ANOVA, 49, 116

stratigraphy, 89, 105

survey

baseline, xv, 16, 19, 23, 28, 29, 30 , $34,35,51,59,62,69,74,87,120$, 127

bathymetry, xiv, xv, 14, 16, 19, 23 , $24,25,28,29,30,32,34,59,62$, $67,69,87,89,97,119,120,123$, $129,131,132,133$

postdisposal, 74

REMOTS $^{\circledR}, 35,120,127$

temperature, 32

tide, $11,16,32,51,52,124$

topography, xiv, xv, 3, 5, 7, 25, 29, 59, $62,67,69,87,89,119,120,123$, $129,131,132,133$

trace metals, 2, 47 arsenic (As), 1, 5, 7, 14, 16, 35, 42, $45,53,54,55,69,84,87,89,97$, $102,111,116,129$

trough, $59,67,89$

turbidity, 67

volume

estimate, 16

waste, 11

waves, 1,32 


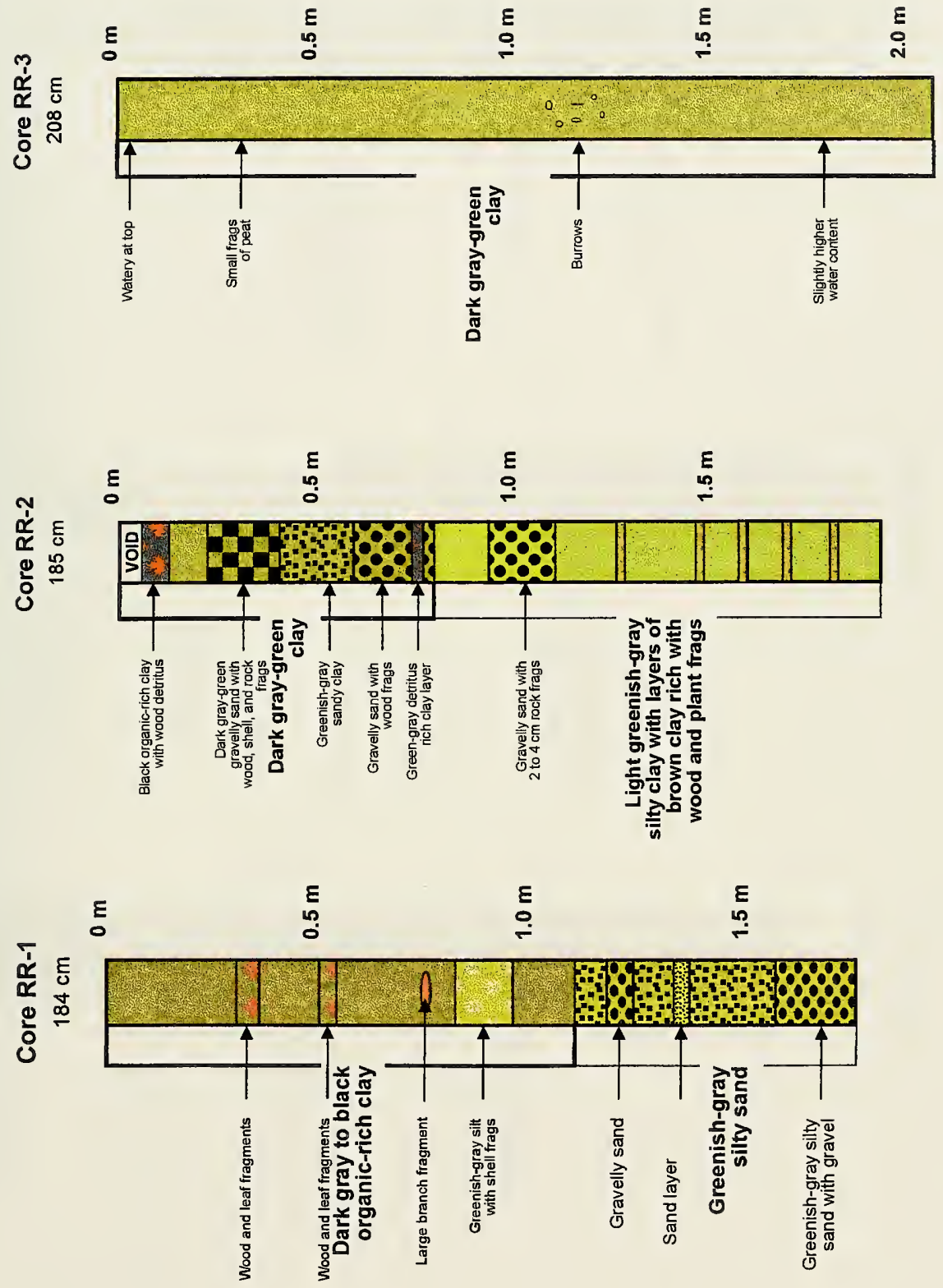


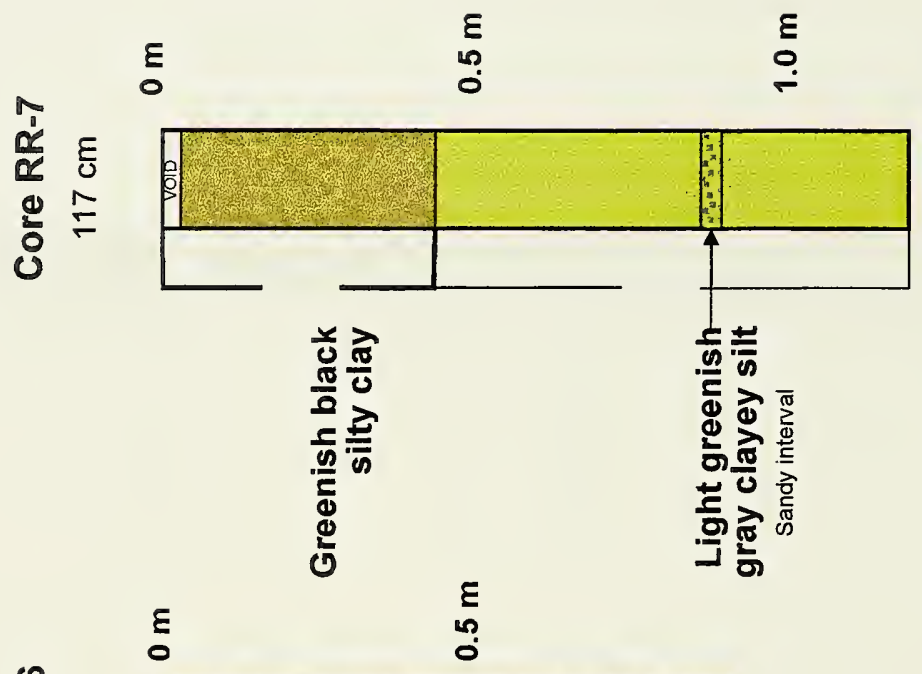

$\begin{array}{ll}\frac{1}{1} & \frac{E}{0} \\ 0 & \sigma \\ \frac{1}{0} & \end{array}$

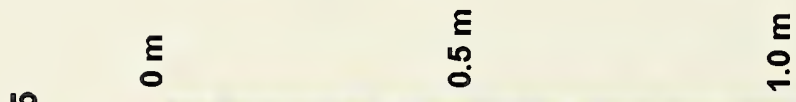

$\frac{0}{\alpha}$
$\frac{1}{0}$
0
0

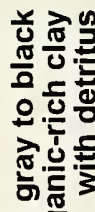

는 응

交

믕

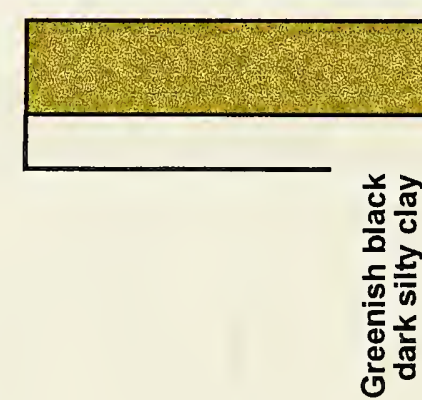

웅
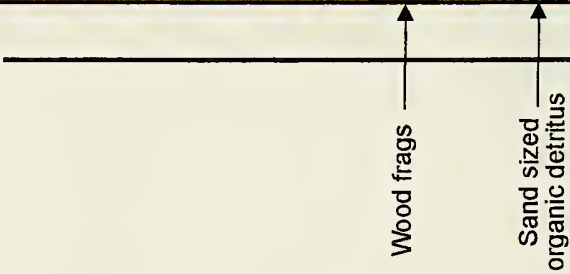

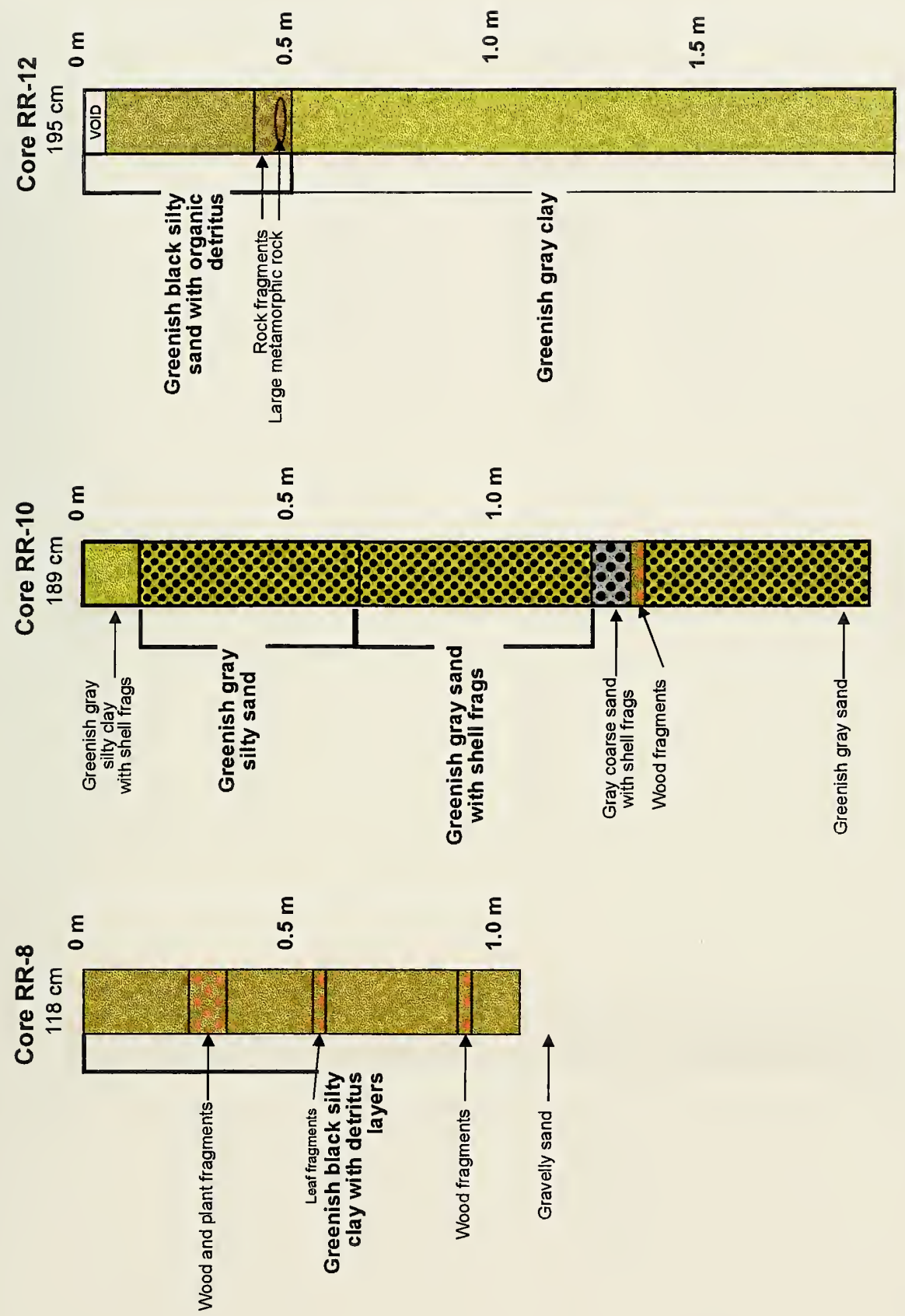

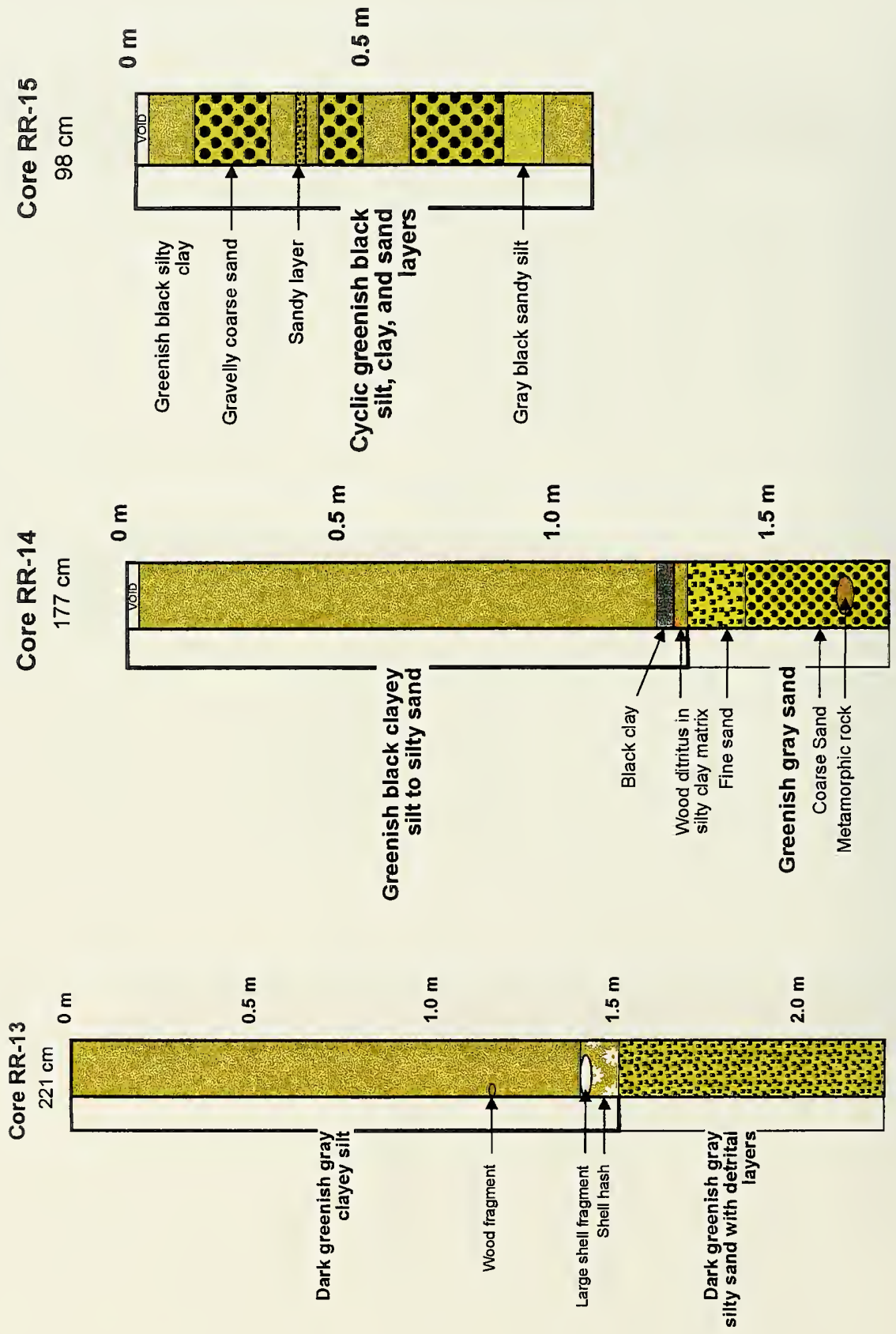

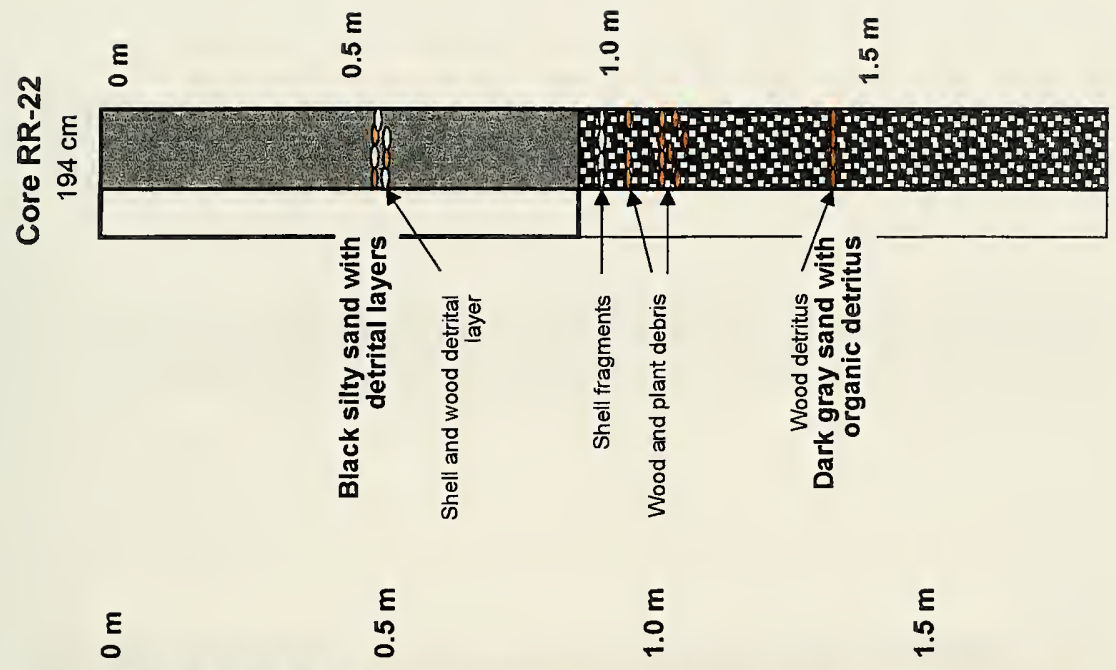

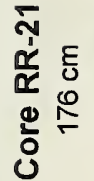

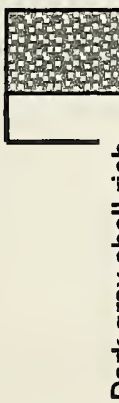

?

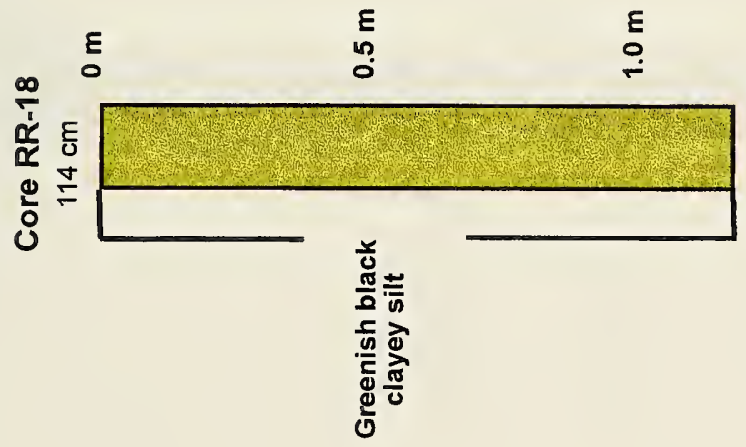



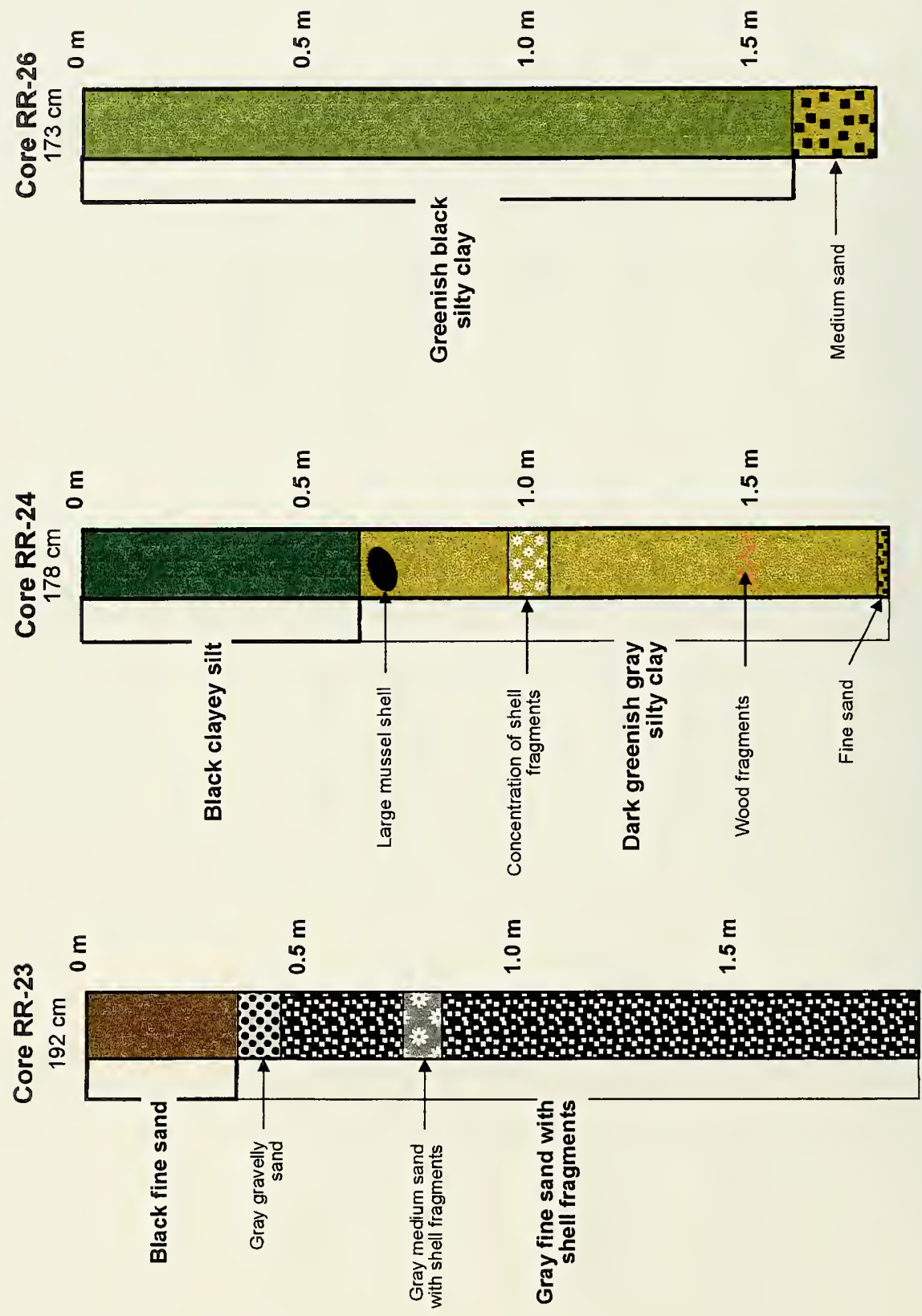

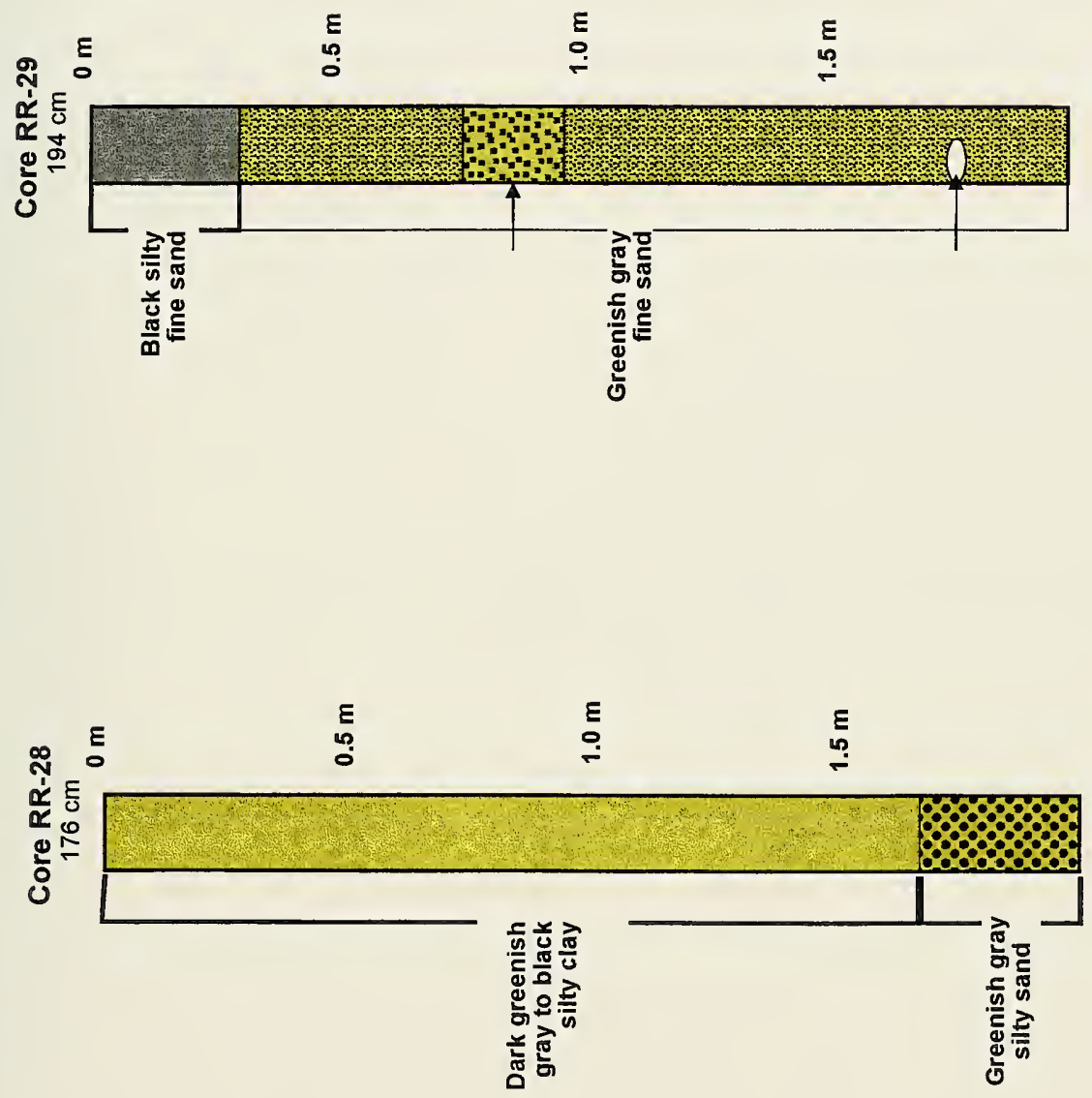


\section{.}


Appendix $B$

Microfossil Results 



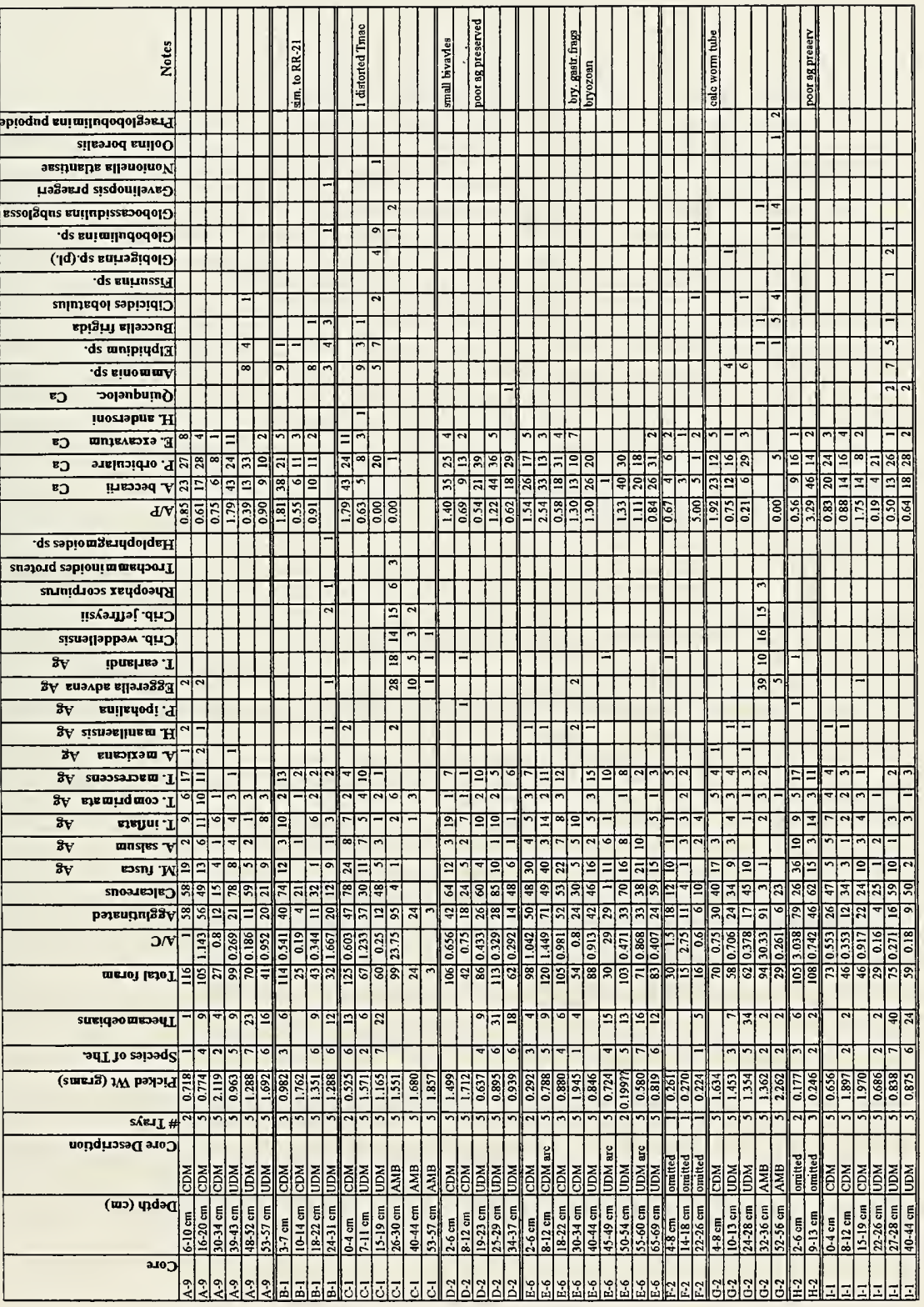




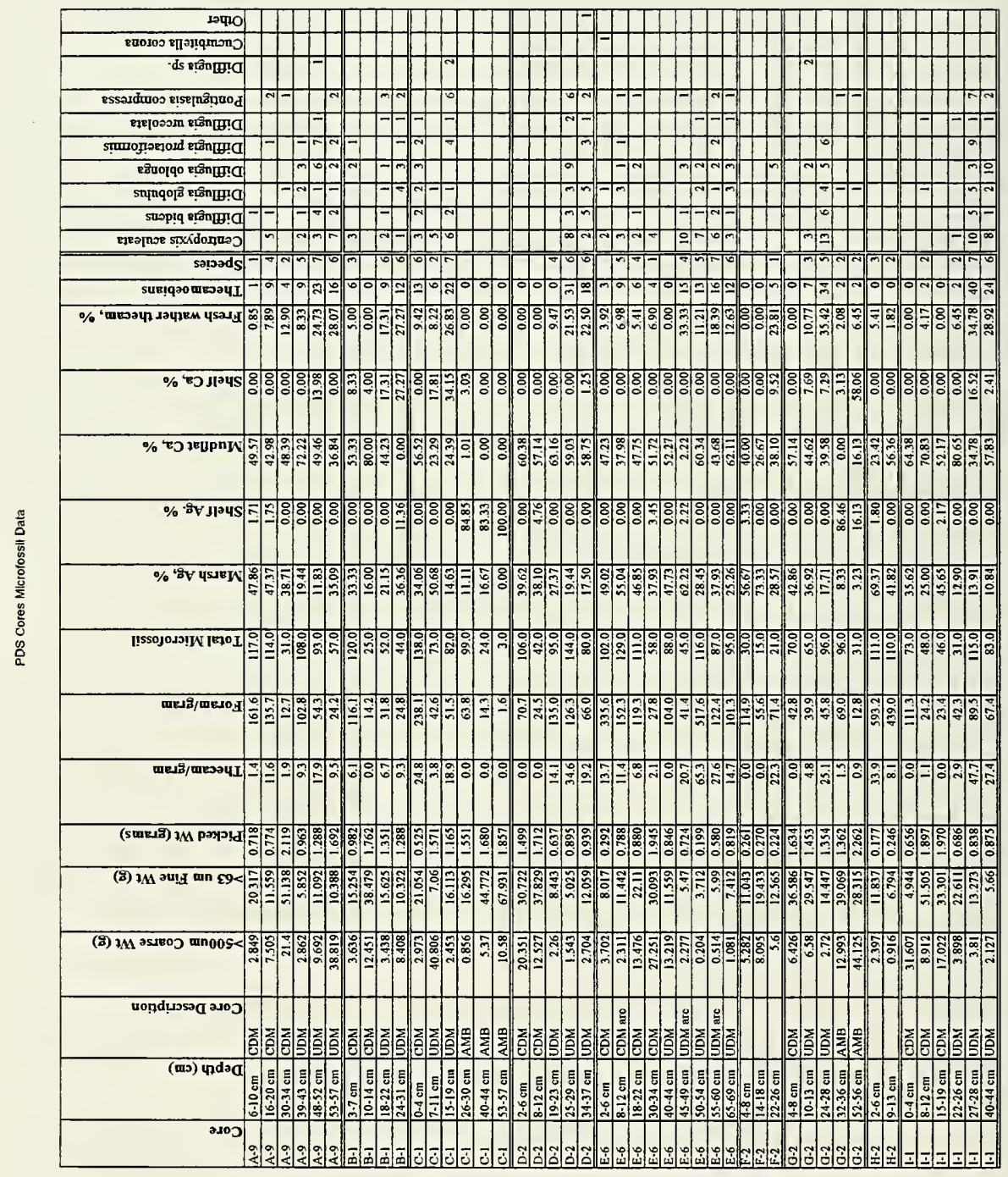




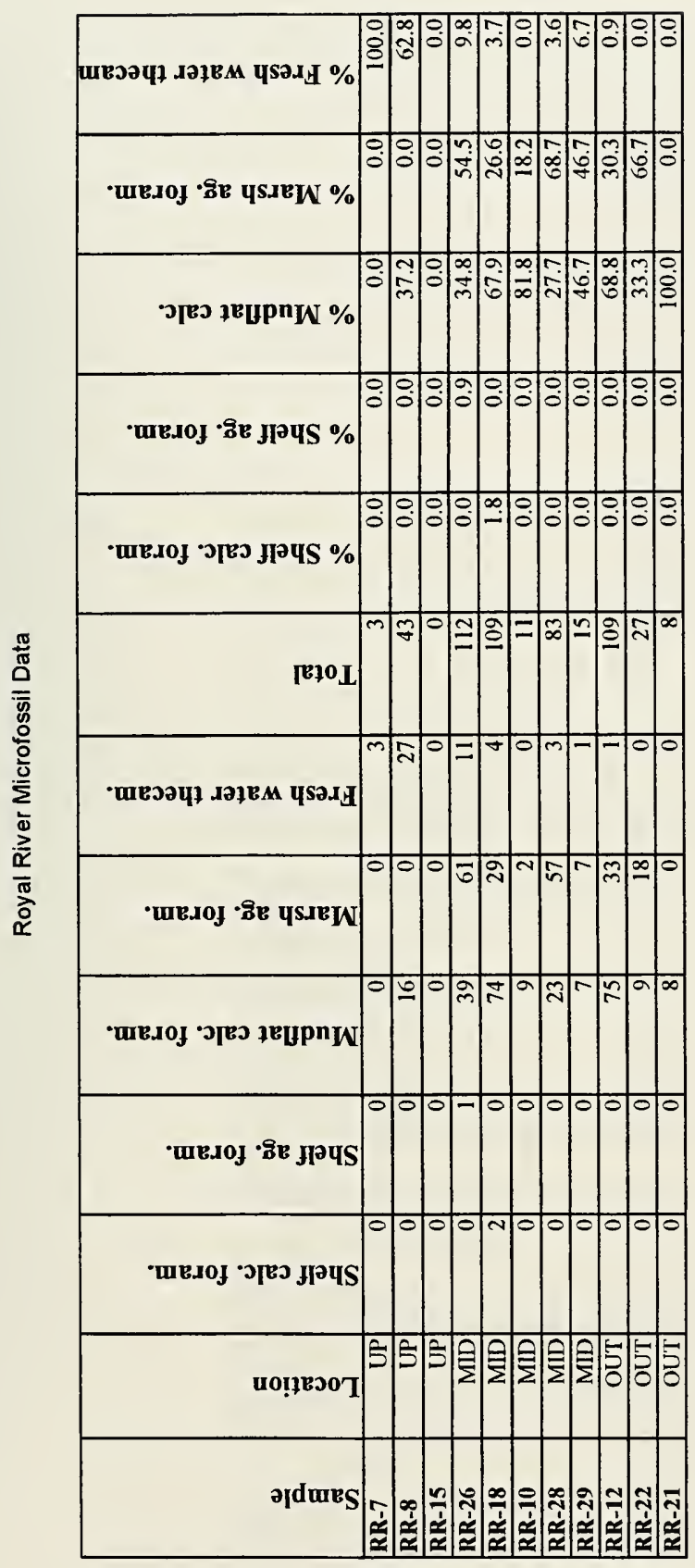




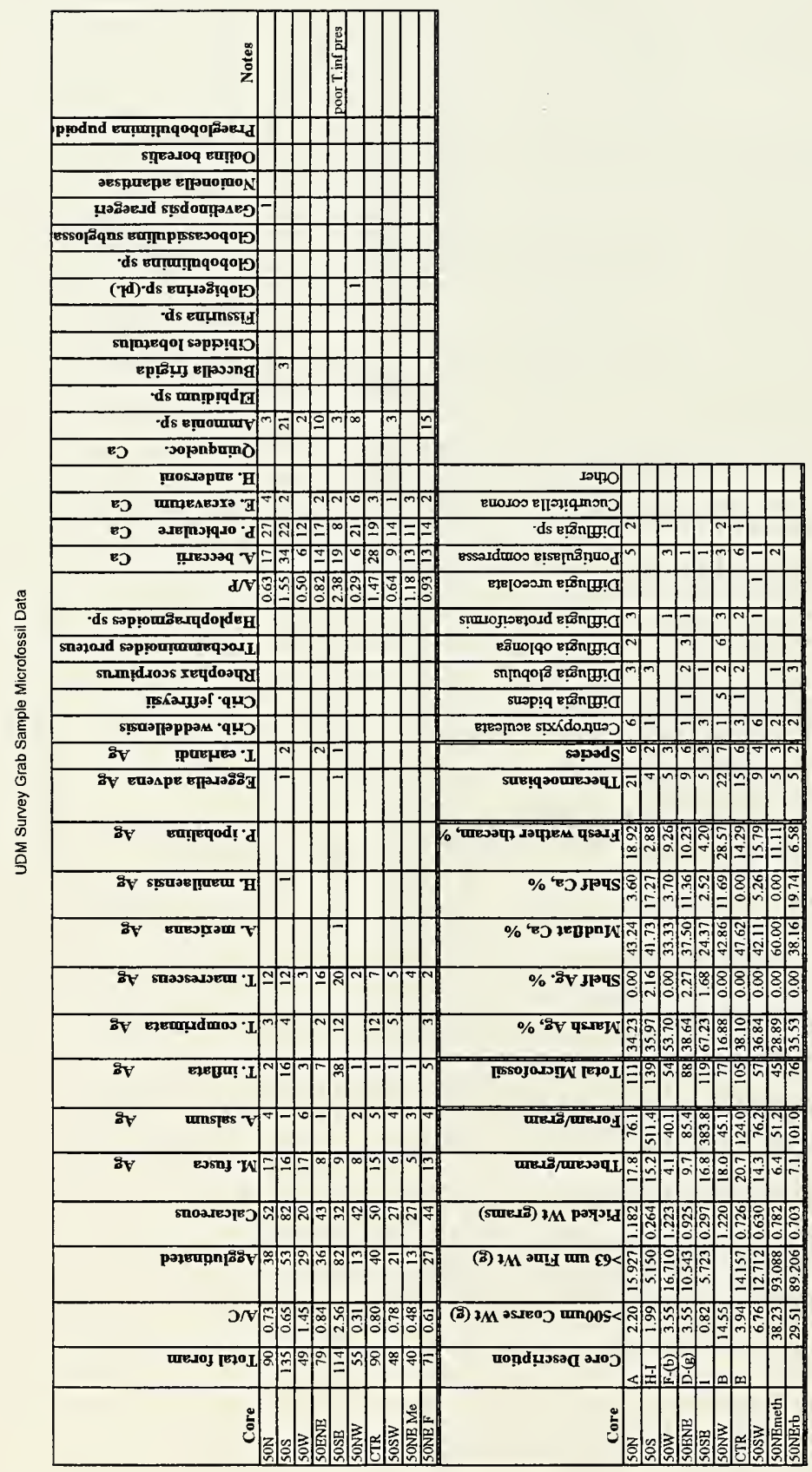


Appendix C

Graphical Representations of Postcap PDS Core Descriptions 



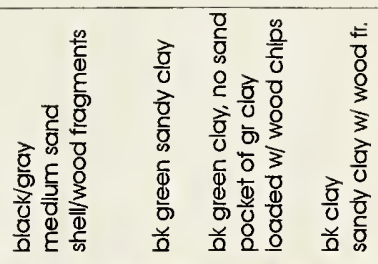

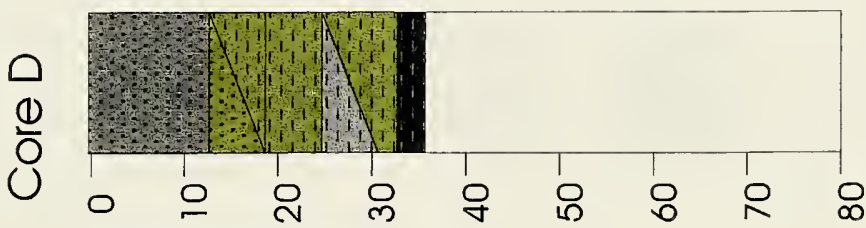

CDM $\mid$ UDM

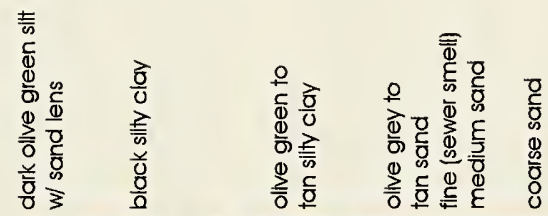

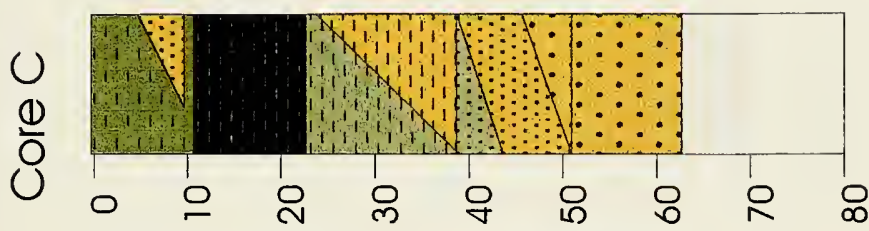
\begin{tabular}{|l||l}
\hline CDM UDM & AMB
\end{tabular}

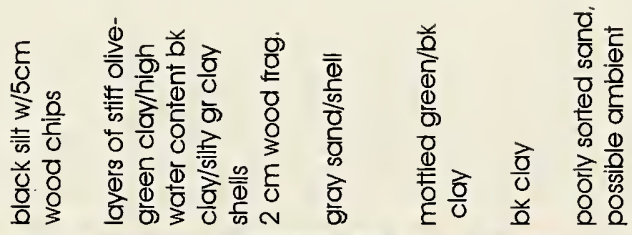

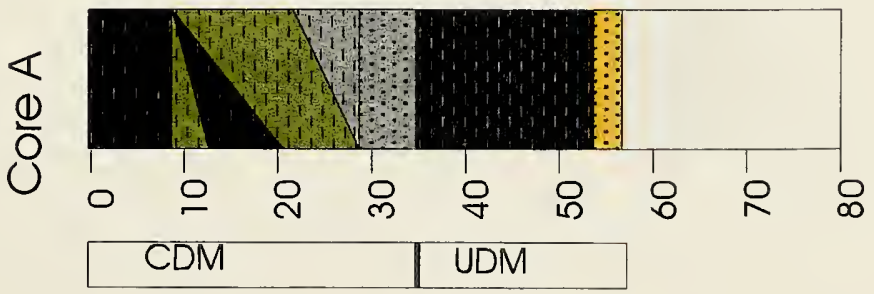




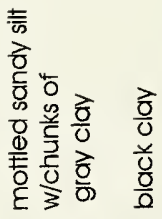

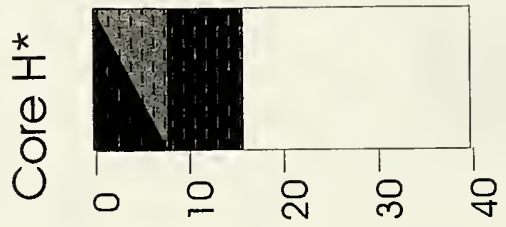

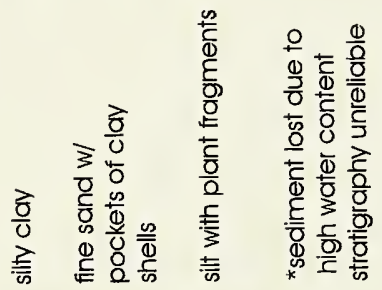

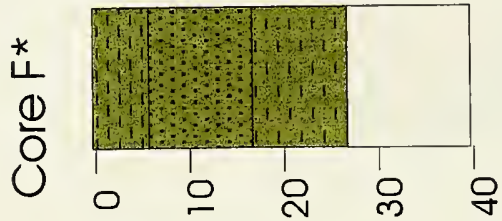

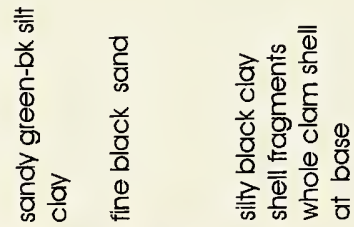

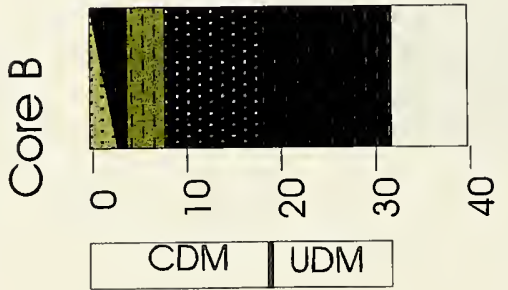




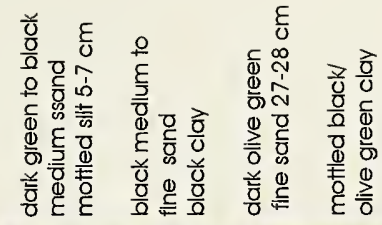
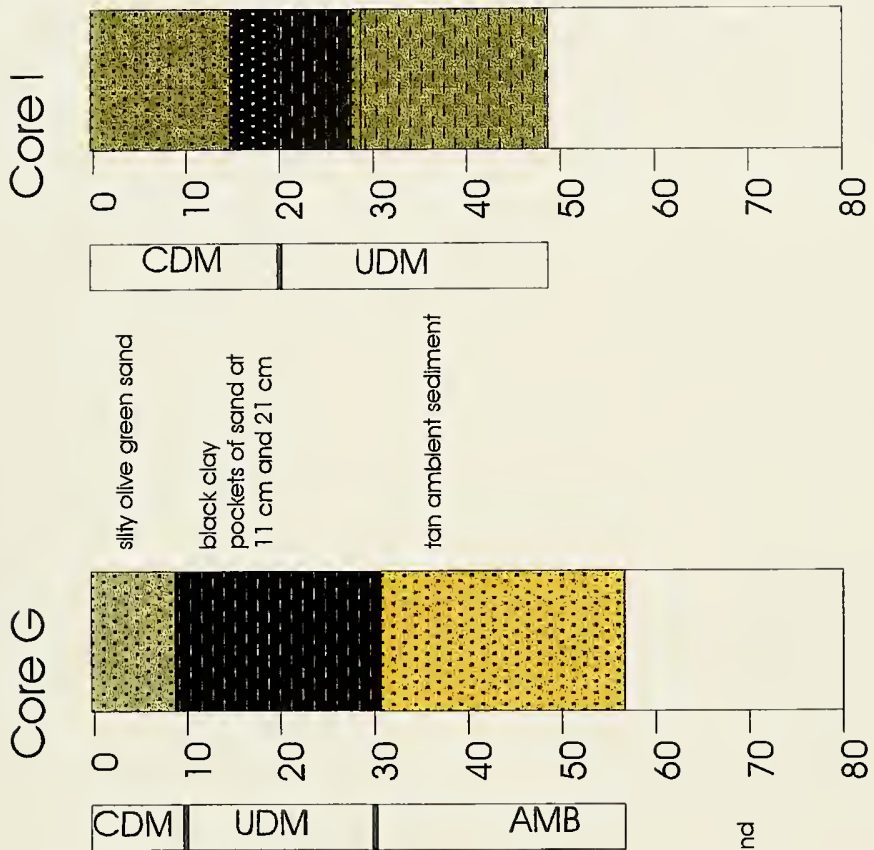

등
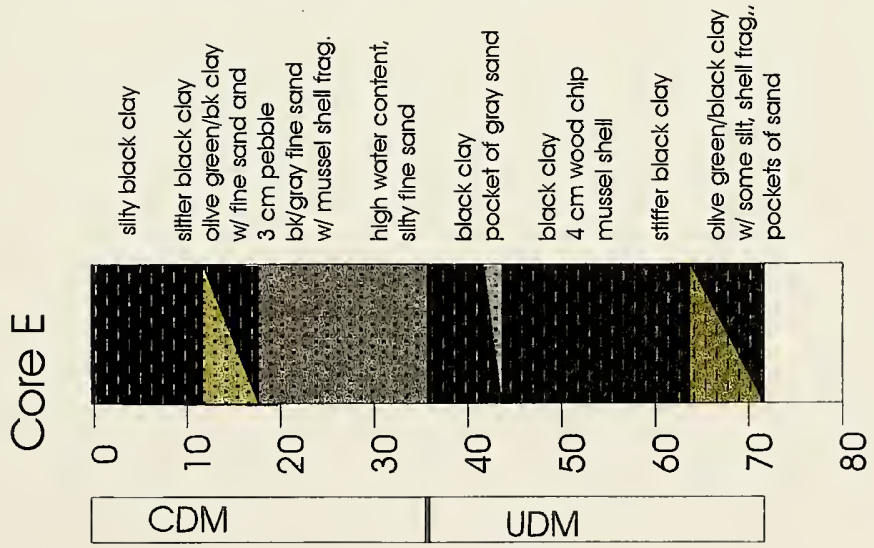

Appendix D

Histograms of Microfossil Results from Postcap Cores 


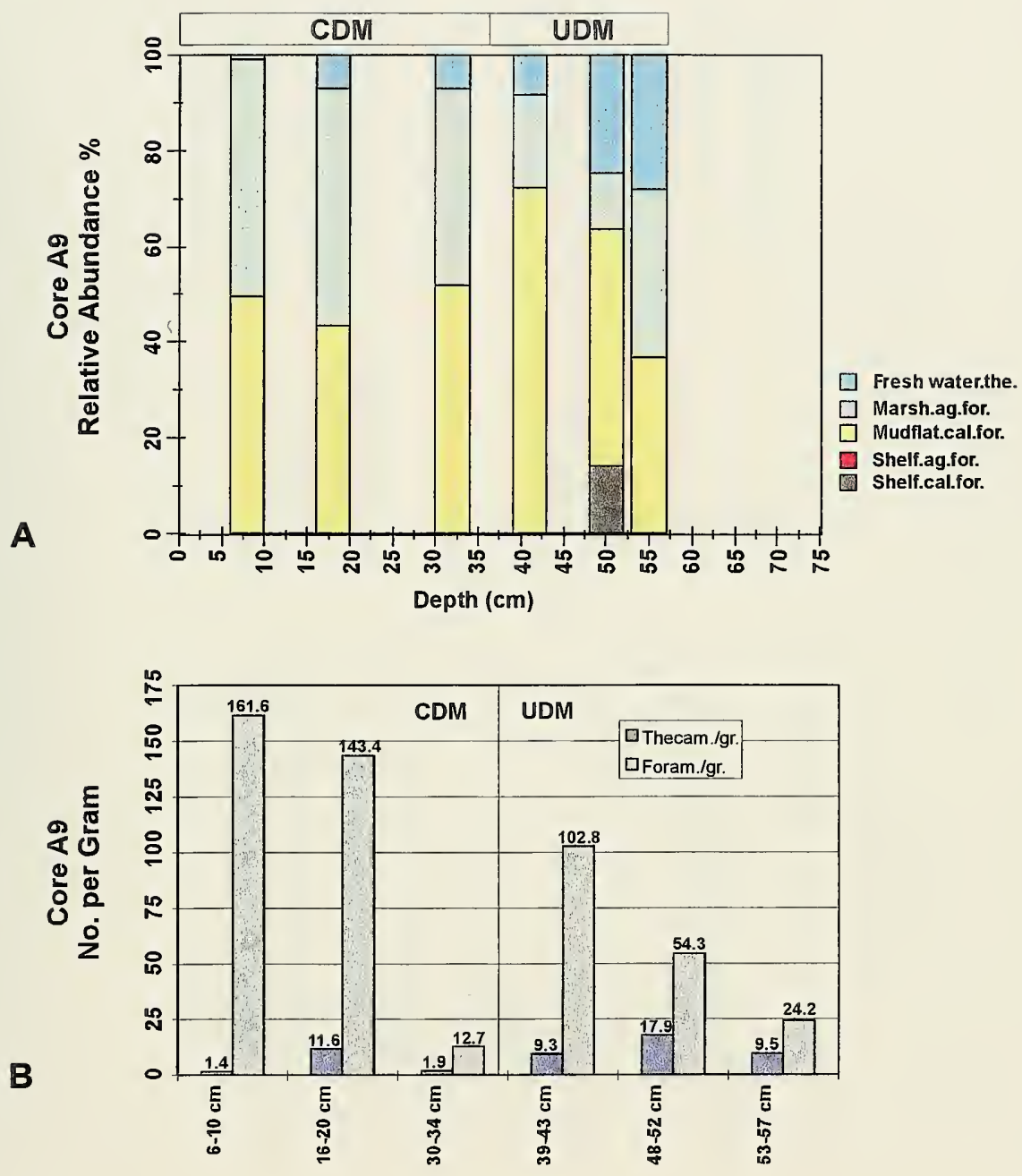

Histograms of the relative abundance and the density of microfossils for each Core A sample. The top bar displays the depth of the cores and identified CDM and UDM layers. 

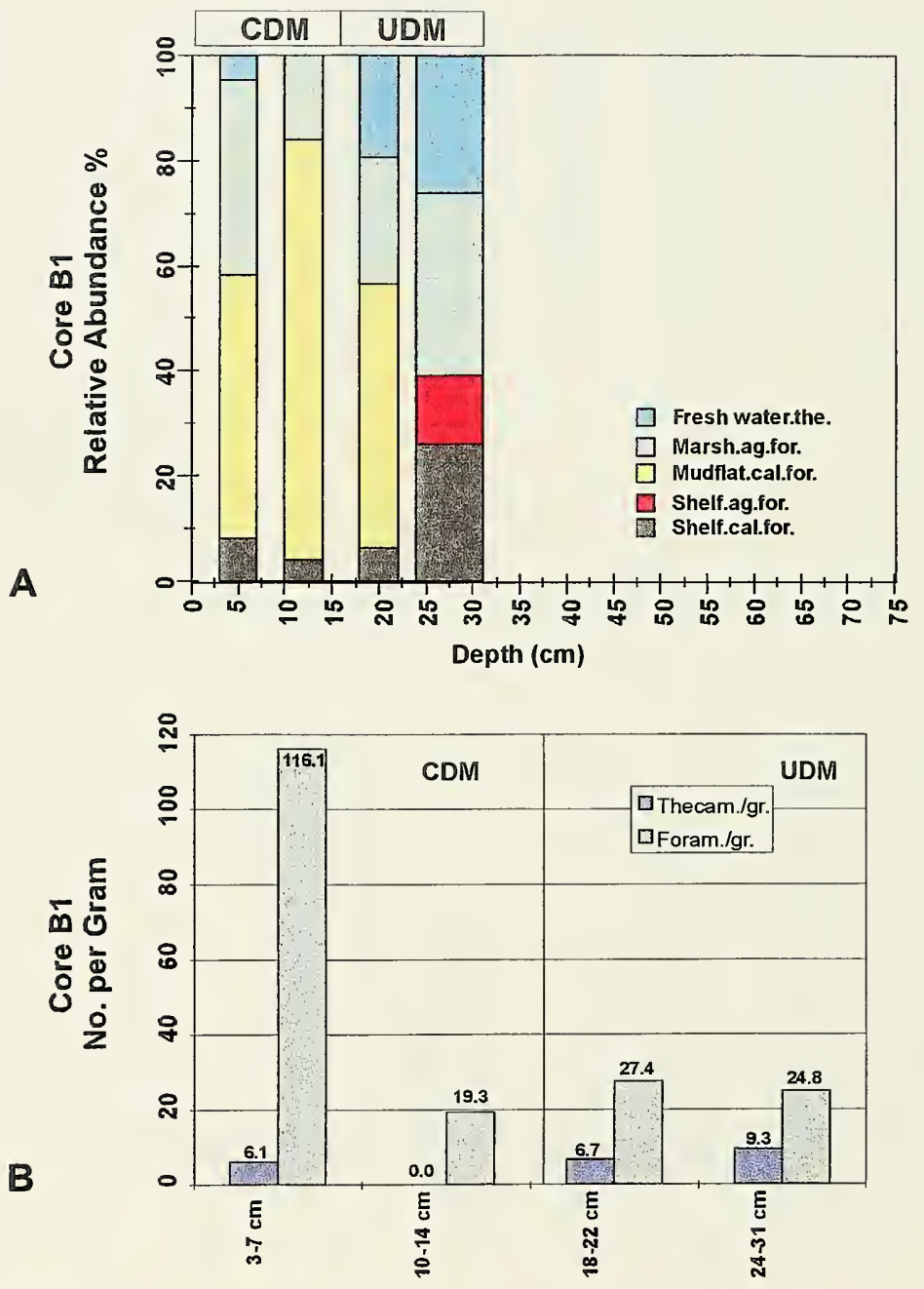

Histograms of the relative abundance and the density of microfossils for each Core B sample. The top bar displays the depth of the cores and identified $\mathrm{CDM}$ and UDM layers. 

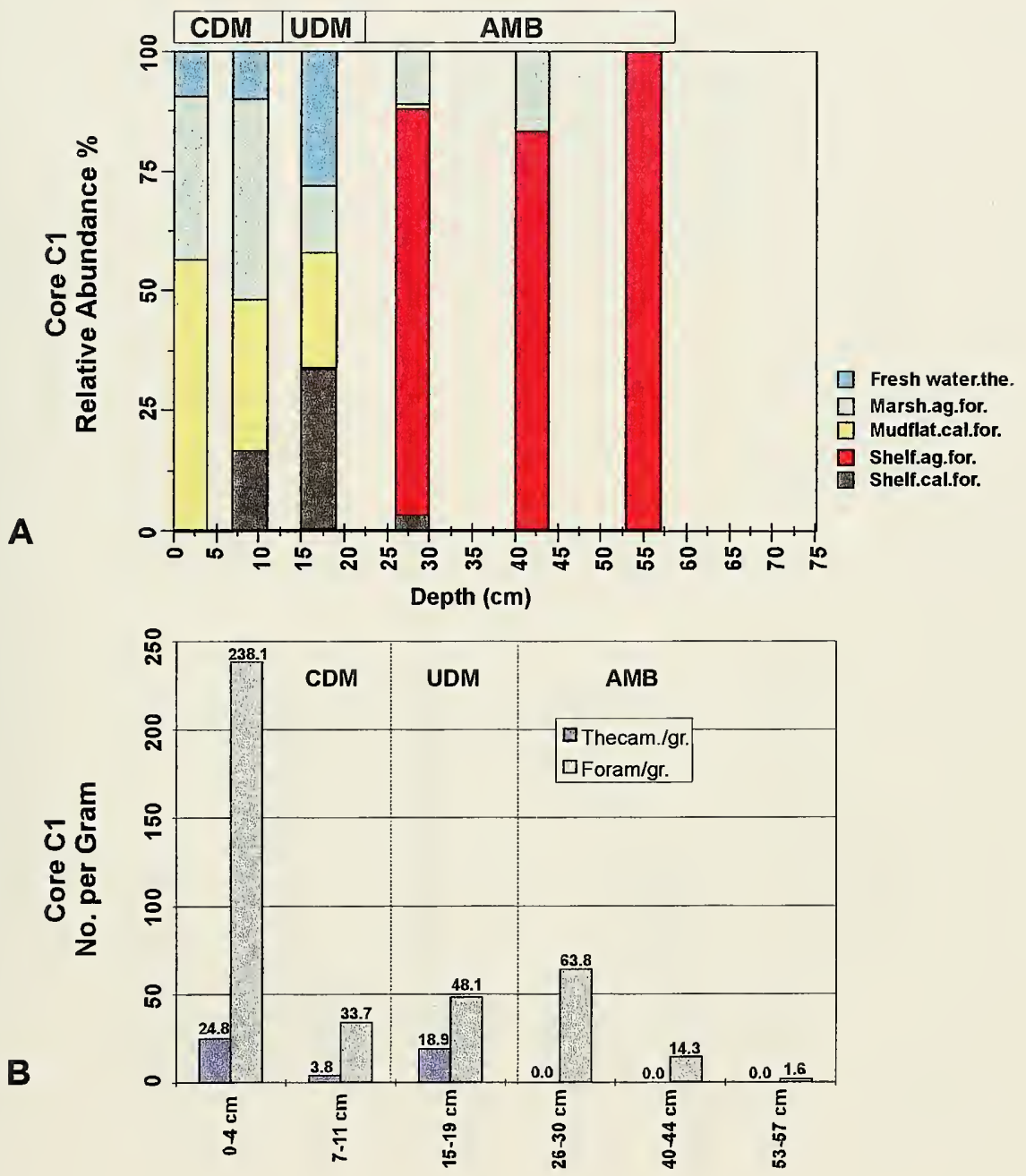

Histograms of the relative abundance and the density of microfossils for each Core $\mathrm{C}$ sample. The top bar displays the depth of the cores and identified CDM, UDM, and ambient layers. 

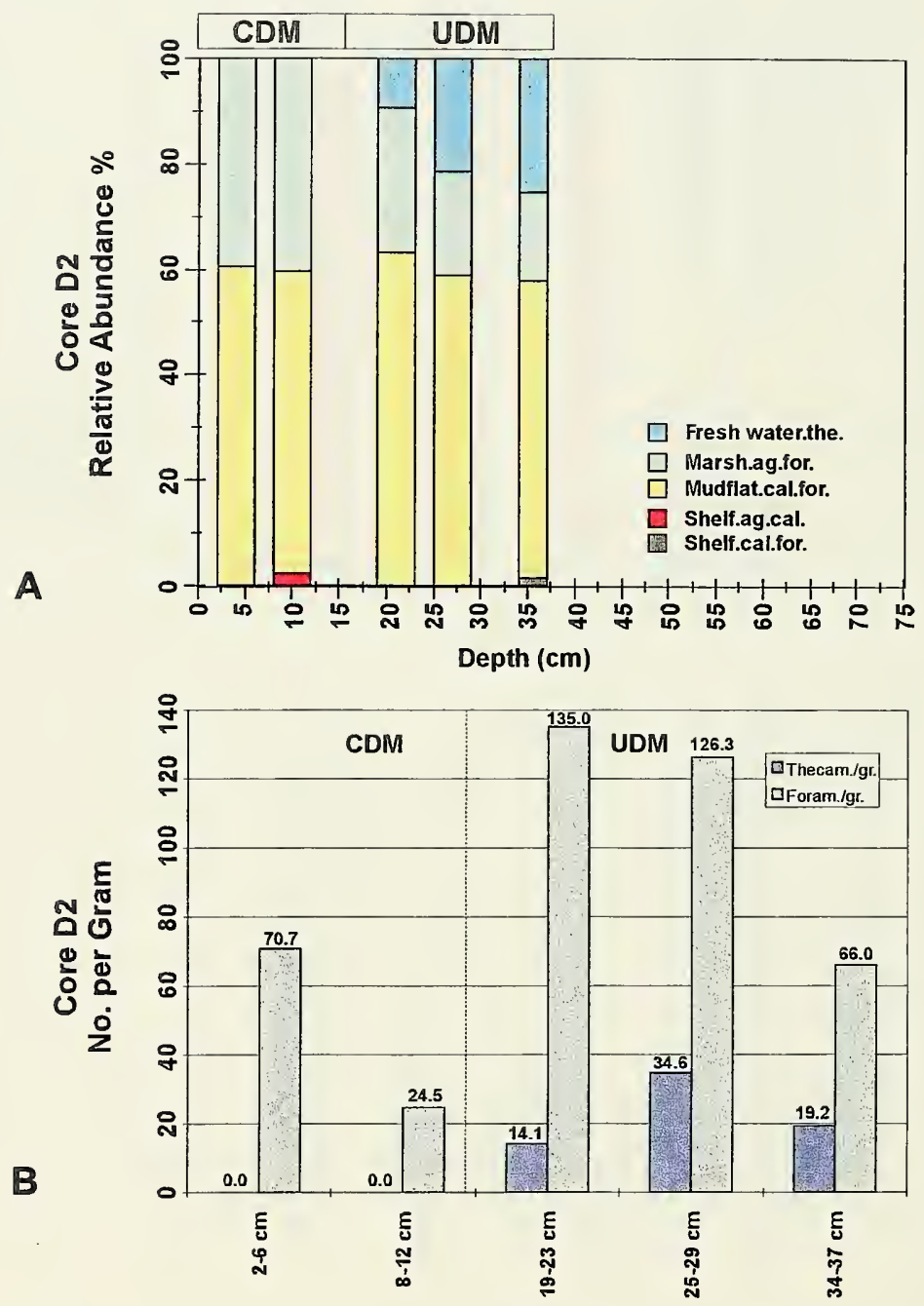

Histograms of the relative abundance and the density of microfossils for each Core $\mathrm{D}$ sample. The top bar displays the depth of the cores and identified CDM and UDM layers. 


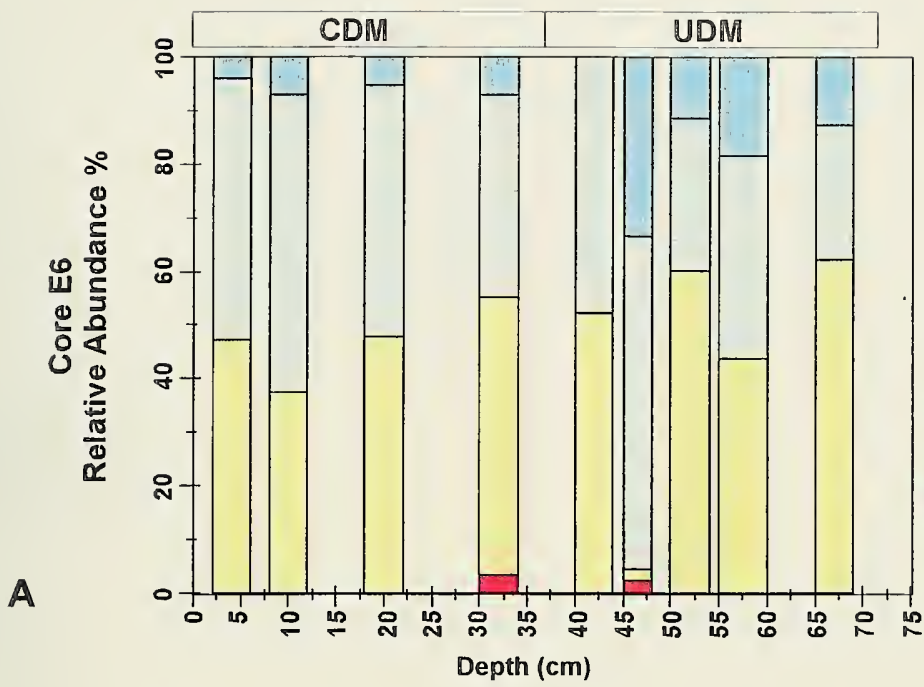

$\square$ Fresh water.the.

$\square$ Marsh.ag.for.

Mudflat.cal.for.

Shelf.ag.cal.

Shelf.cal.for.

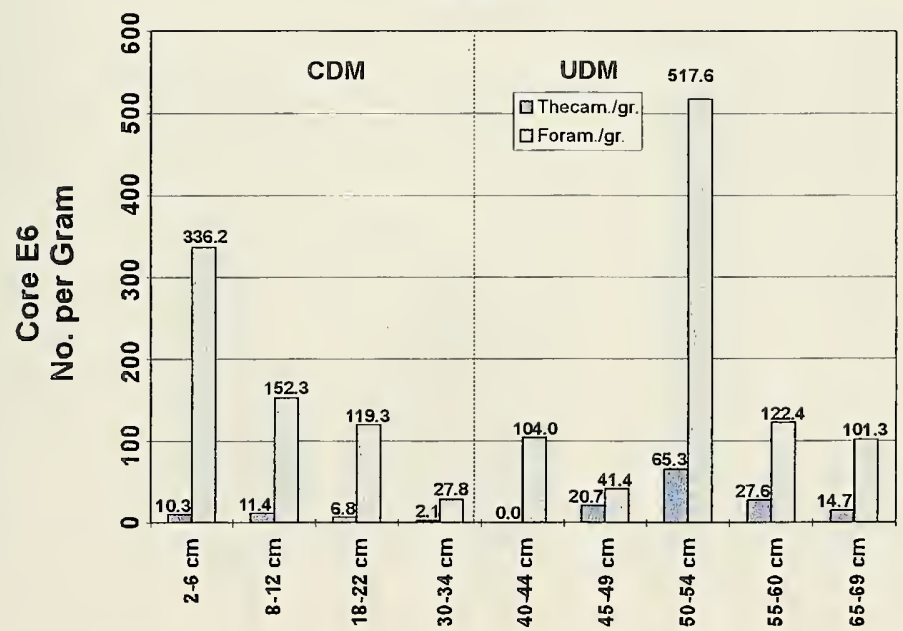

Histograms of the relative abundance and the density of microfossils for each Core E sample. The top bar displays the depth of the cores and identified $\mathrm{CDM}$ and UDM layers. 


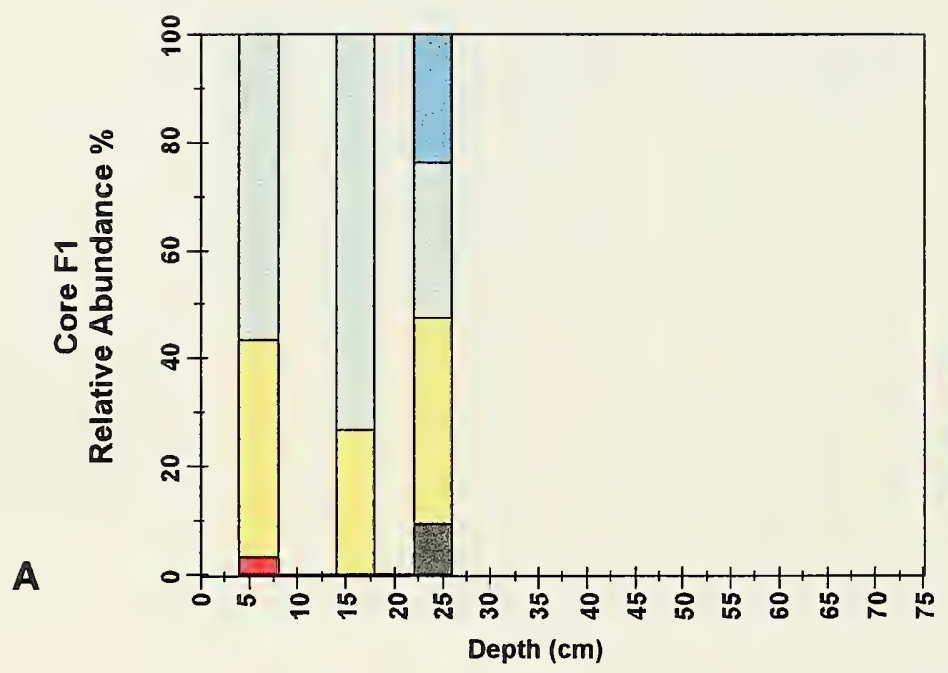

$\square$ Fresh water.the.

$\square$ Marsh.ag.for. Mudflat.cal.for.

Shelf.ag.cal.

Shelf.cal.for.

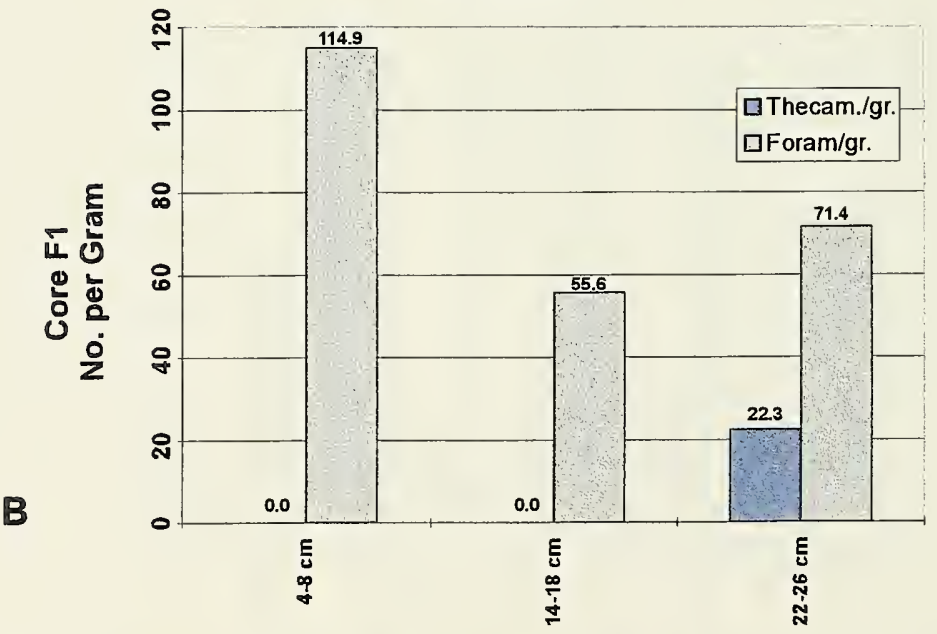

Histograms of the relative abundance and the density of microfossils for each Core F sample. The stratigraphy of the dredged material layers was unreliable due to water-logging of the core. 

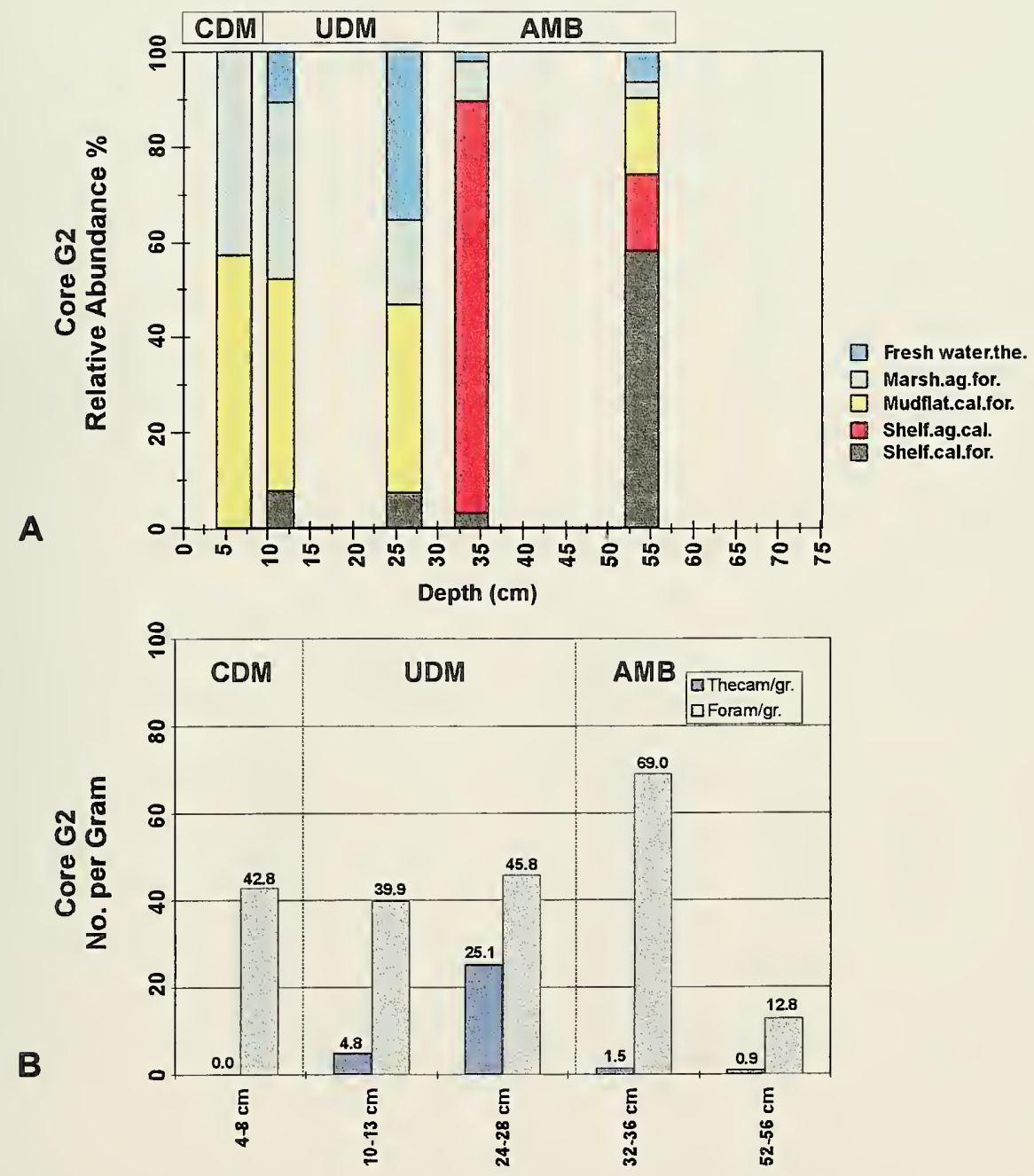

Histograms of the relative abundance and the density of microfossils for each Core $\mathrm{G}$ sample. The top bar displays the depth of the cores and identified CDM, UDM, and ambient layers. 


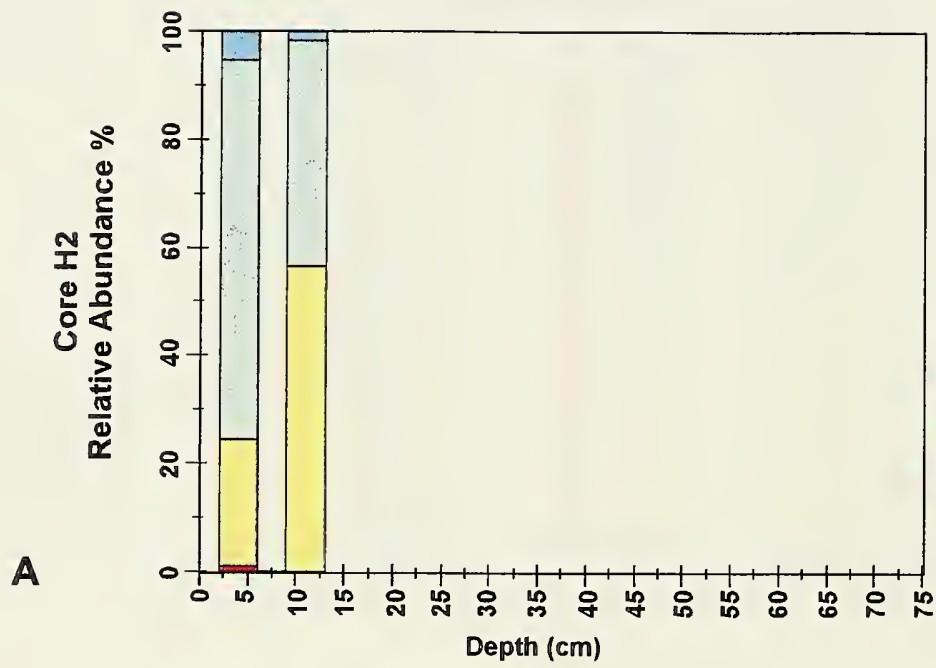

Fresh water.the.

$\square$ Marsh.ag.for.

Mudflat.cal.for.

Shelf.ag.cal.

Shelf.cal.for.

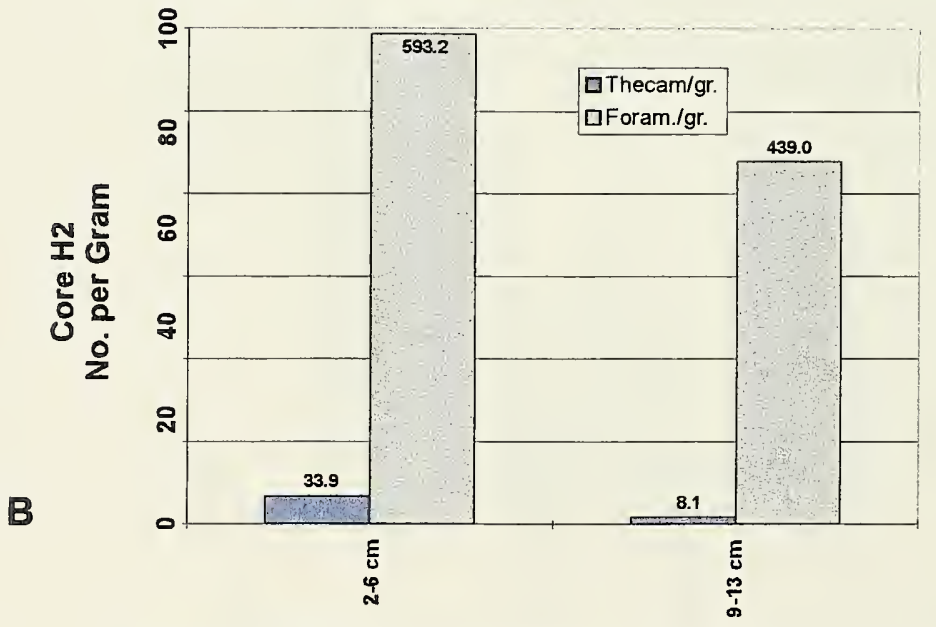

Histograms of the relative abundance and the density of microfossils for each Core $\mathrm{H}$ sample. The stratigraphy of the dredged material layers was unreliable due to water-logging of the core. 

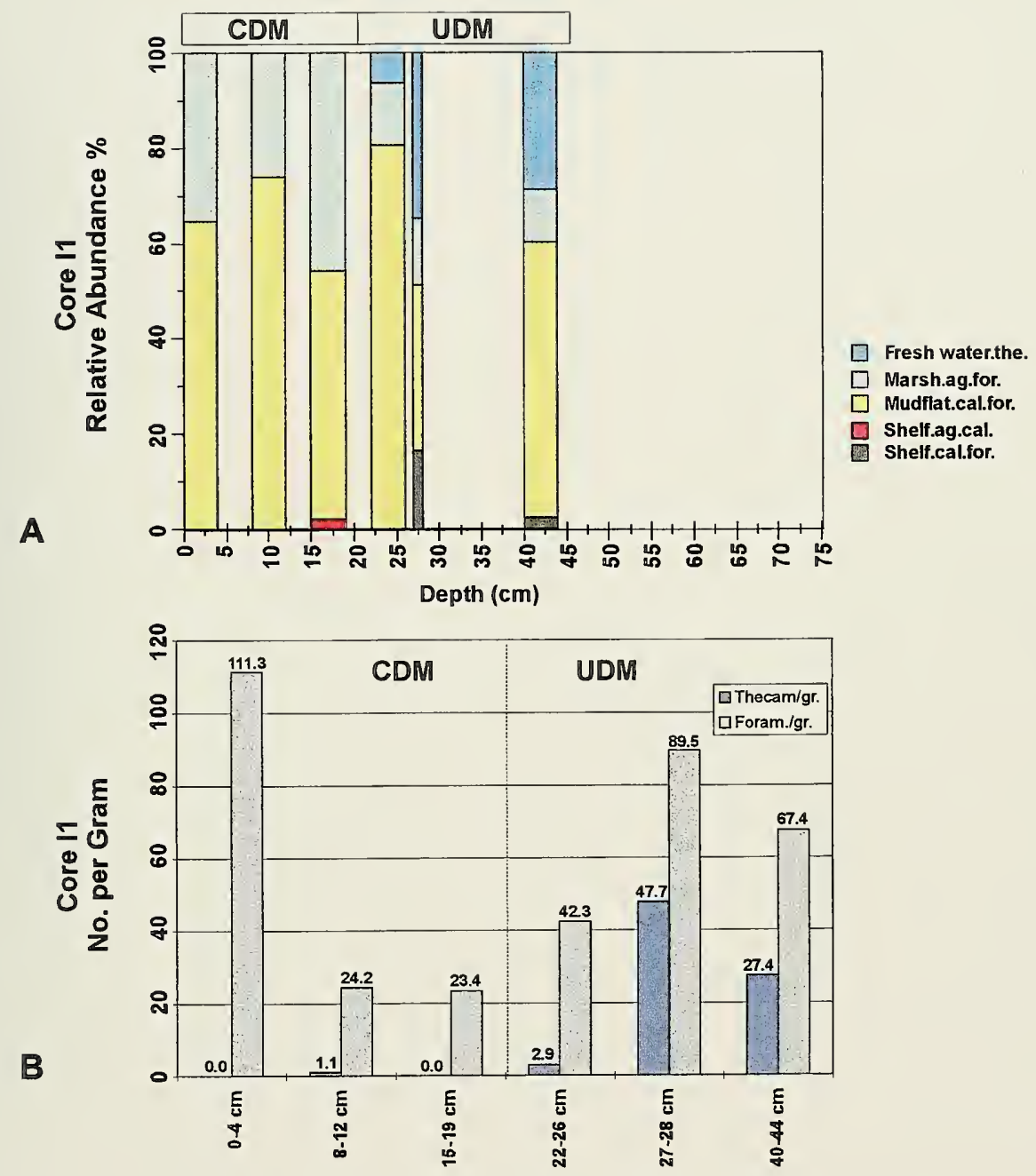

Histograms of the relative abundance and the density of microfossils for each Core I sample. The top bar displays the depth of the cores and identified CDM and UDM layers. 


